\title{
Non-Contact Bed-Based Monitoring of Vital Signs
}

by

Stephanie Bennett, B.Eng, M.A.Sc.

A thesis submitted to the Faculty of Graduate and Post Doctoral Affairs in partial fulfillment of the requirements for the degree of

A Doctor of Philosophy in Electrical and Computer Engineering

Ottawa-Carleton Institute for Electrical and Computer Engineering

Department of Systems and Computer Engineering

Carleton University

Ottawa, Ontario, Canada, K1S5B6

May 30, 2019

(C) Copyright 2019 Stephanie Bennett 


\section{Abstract}

Vital signs are a set of commonly measured signals used internationally as a baseline in medicine and surgery and are one of the most accurate predictors of clinical and physiological deterioration. Despite the clear clinical importance of vital sign measurements, there is often missed or inadequate documentation of patient vital sign measurements. The development of unobtrusive, automated and continuous monitoring offers the potential to enhance the safety and quality of patient care.

This thesis details a system that uses multiple modalities to capture data and data processing techniques to extract vital signal measurements and vital signal measurement abnormalities related to subject morbidities. This thesis focuses on the examination, testing and improvement upon a current visible light video processing technique intended to extract vital signal measurements, and expand it's use to thermal infrared video vital signal extraction.

Three modalities were used to gather data from healthy adult subjects and older adult inhospital patients: thermal infrared cameras, visible light cameras and pressure sensitive mats. Subjects participated in several experimental procedures including video data capture of faces, hands and feet as well as in-bed pressure mat data capture. This data was subjected to several stages of data processing to extract vital signal measurements, which include pulse, respiration temperature and mobility measurements. Data segmentation using binary masks, level set method, and watershed method were used to identify regions of interest. An adaptive spatio-temporal video processing algorithm, the main thesis contribution, was used to extract vital signal measurements. The developed algorithm was assessed for its performance in vital signal estimation, as well its 
robustness with respect to noise. The whole system was assessed for its ability to extract vital signal measurements, and vital signal measurement abnormalities associated with possible subject morbidities.

The whole system demonstrated accurate vital signal measurement extraction, and the ability to detect signal abnormalities associated with subject morbidities. The developed algorithm was able to improve upon previous versions. Furthermore, several patterns associated with system failure were identified. This work demonstrated the potential for multiple sensor systems in conjunction with data processing to improve current methods of extracting vital signal data. 


\section{Acknowledgements}

Thank you to AGE-WELL NCE Inc., a member of the Networks of Centres of Excellence program, as well as the National Sciences and Engineering Research Council (NSERC) of Canada for funding and support throughout my degree.

Thank you to my academic supervisor, Dr. Rafik Goubran and my medical supervisor, Dr. Frank Knoefel, for the enormous support I received throughout my thesis. I am forever grateful for their professional mentoring, for their confidence in my work and work ethic, and for all their time and effort given always without hesitation.

Thank you to my lab co-habitants Vilas Joshi and Madison Cohen-McFarlane for being good sounding boards and even better friends, and Bruce Wallace for meeting me on his own time and answering my endless questions.

Finally, I would like to thank my family and dear friends for giving me so much unconditional love, support and encouragement. Every word of encouragement made a difference, and I would not be where I am without them. This thesis is theirs as well as mine. 


\section{Table of Contents}

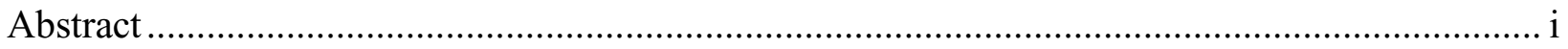

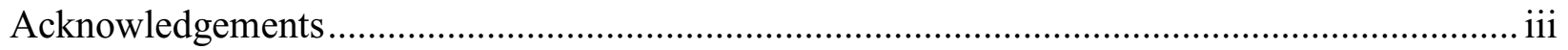

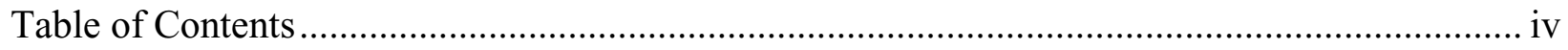

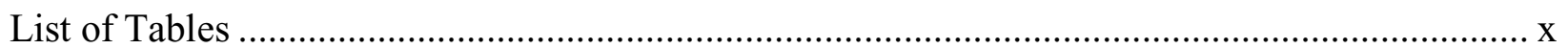

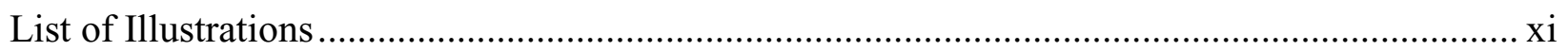

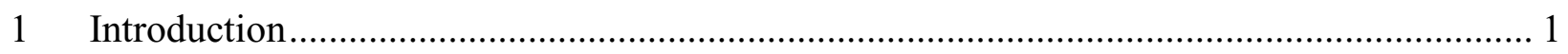

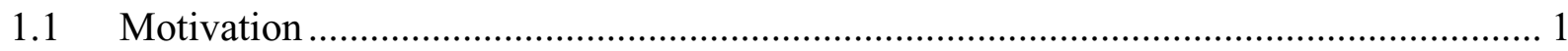

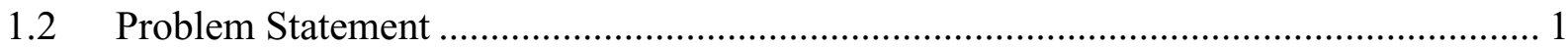

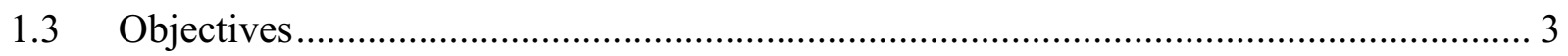

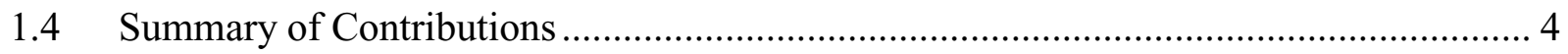

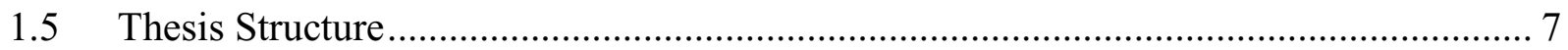

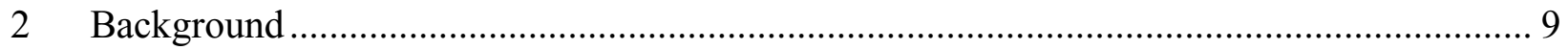

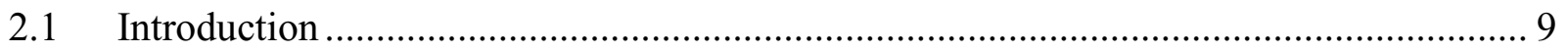

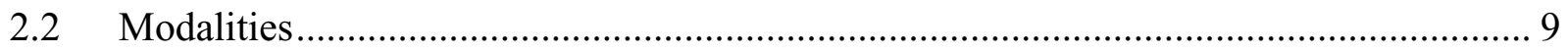

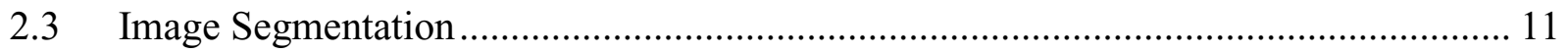

2.4 Eulerian Video Magnification........................................................................... 12

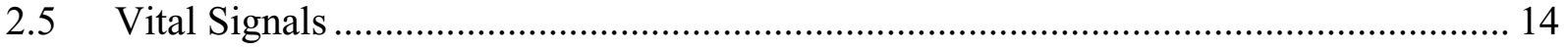




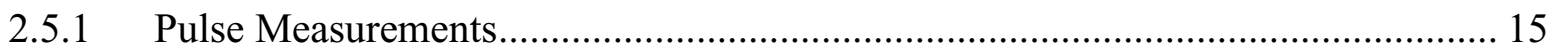

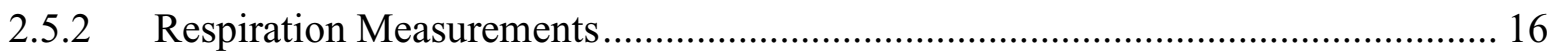

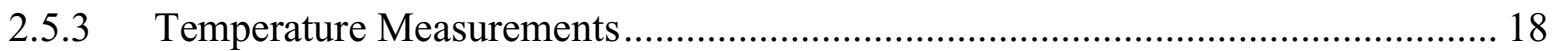

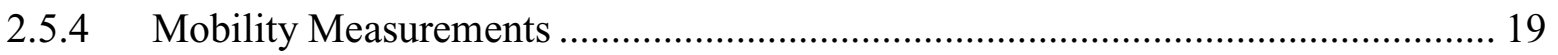

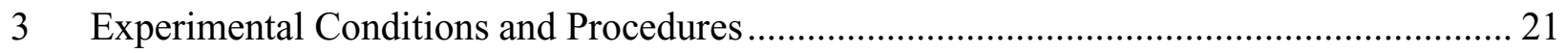

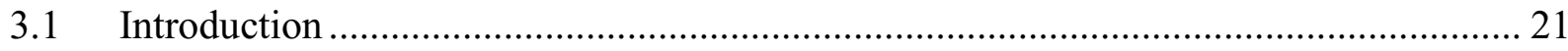

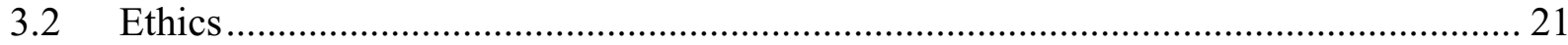

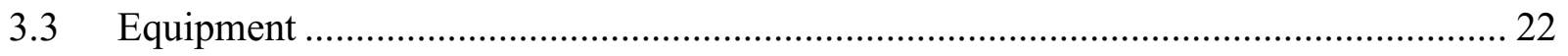

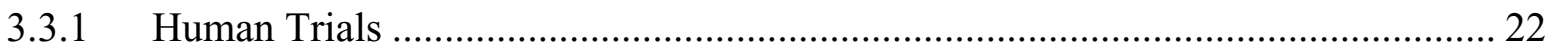

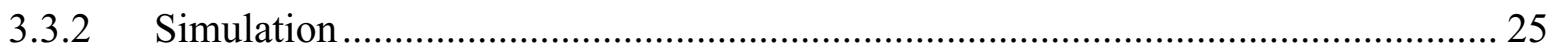

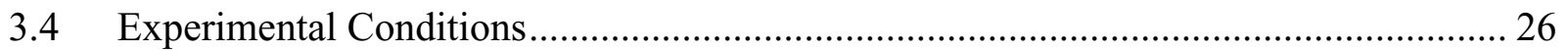

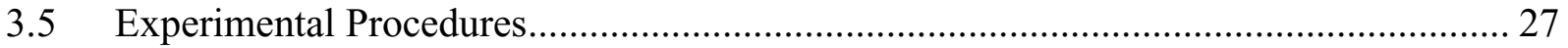

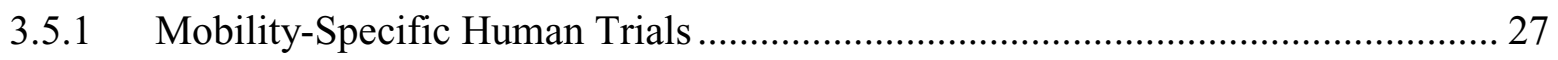

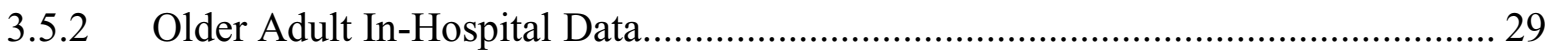

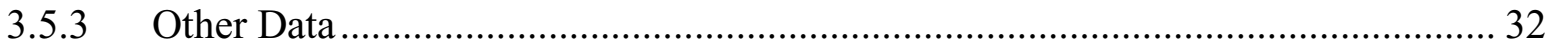

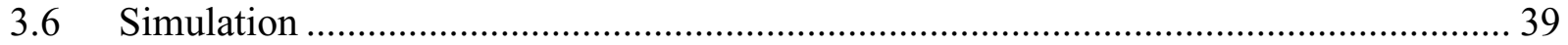

4 Data Preprocessing and Context Awareness in Vital Sign Estimation................................... 40

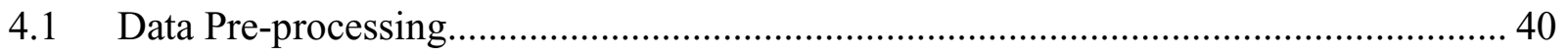

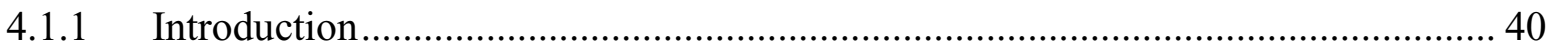

4.1.2 Thermal Infrared Preprocessing ......................................................................... 41 


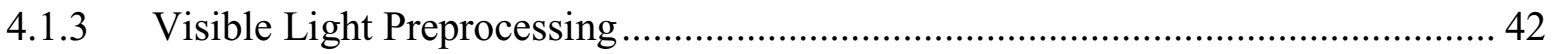

4.1.4 Pressure Sensor Signal Preprocessing .............................................................. 42

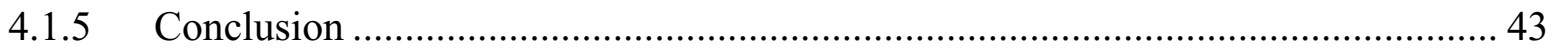

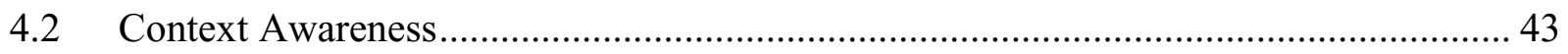

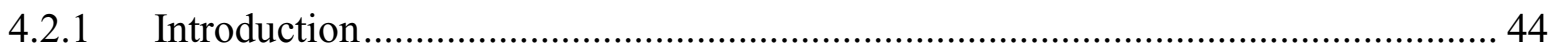

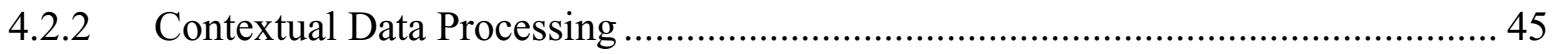

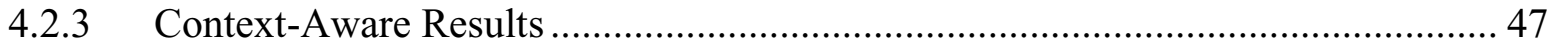

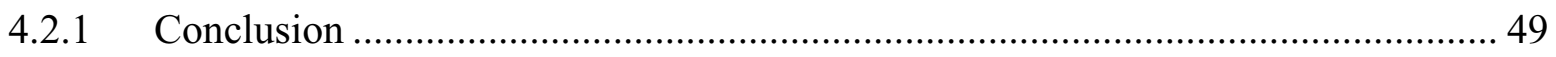

5 Data Segmentation in Vital Sign Estimation ................................................................ 50

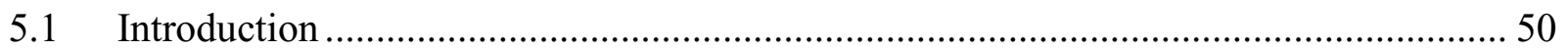

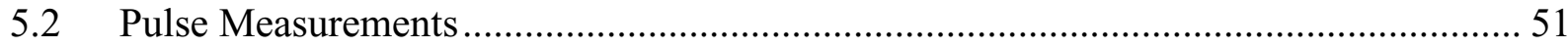

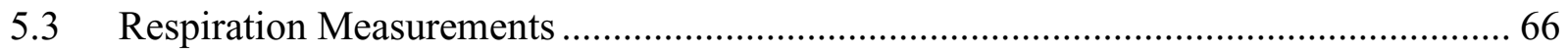

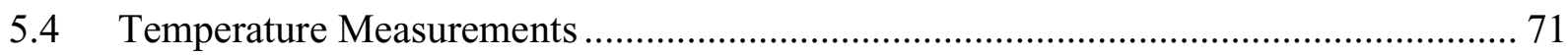

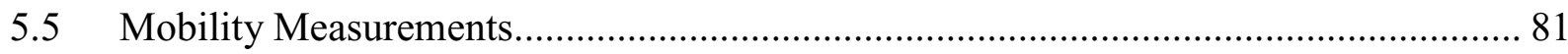

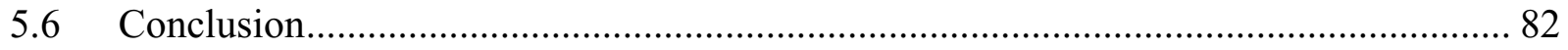

6 Video Magnification in Vital Signal Extraction ................................................................ 85

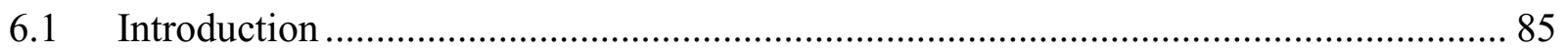

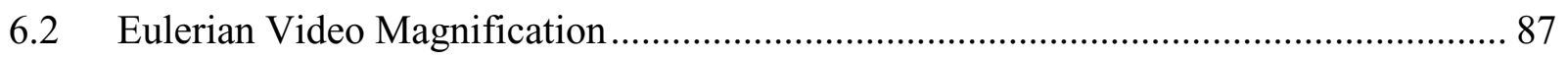

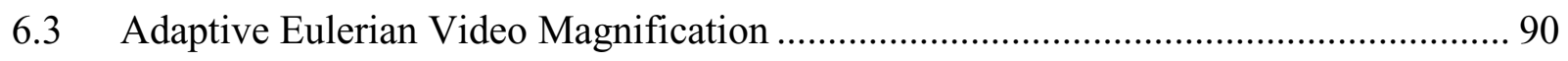

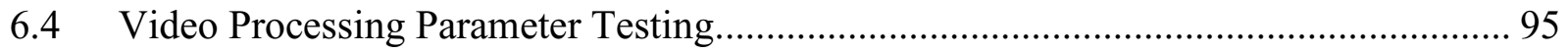




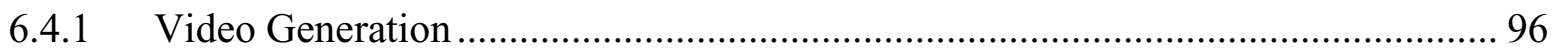

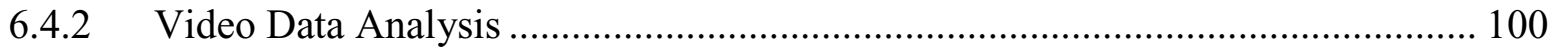

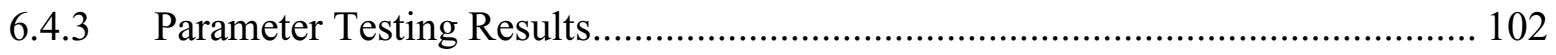

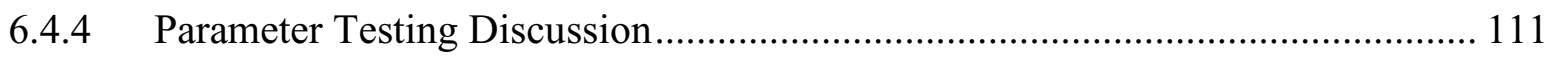

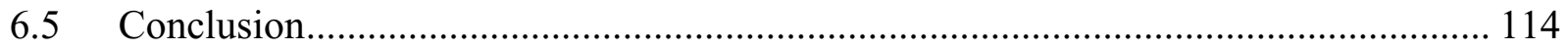

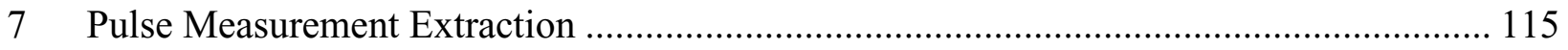

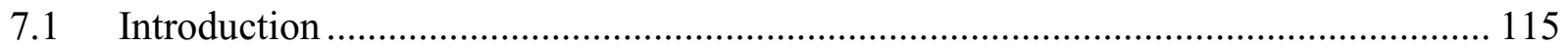

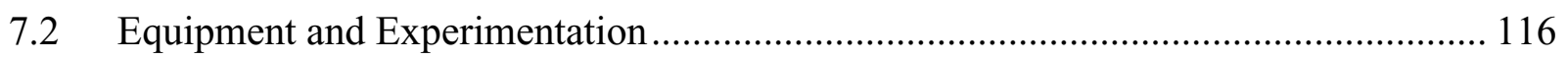

7.3 Pulse Measurement Data Analysis ......................................................................... 117

7.3.1 Thermal Infrared Data Analysis....................................................................... 117

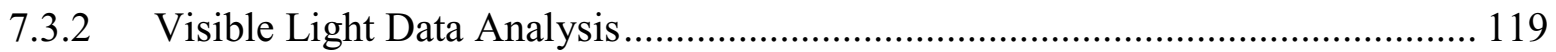

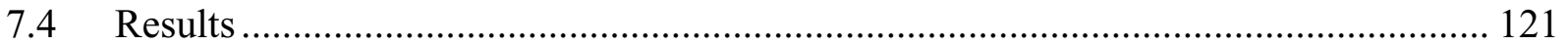

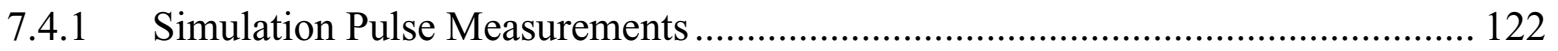

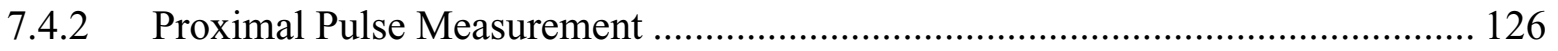

7.4.3 Distal Pulse Measurement............................................................................ 130

7.4.4 Pulse Abnormalities Measurements.................................................................. 133

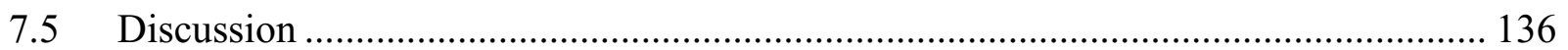

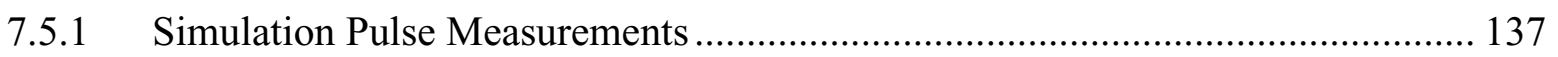

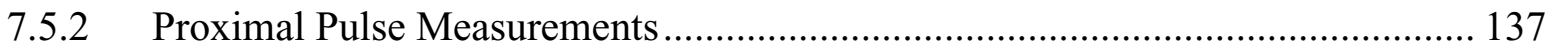

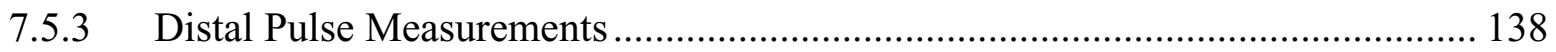




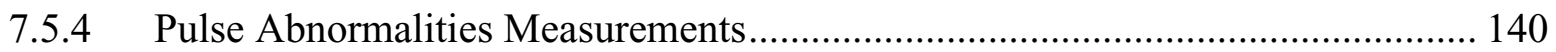

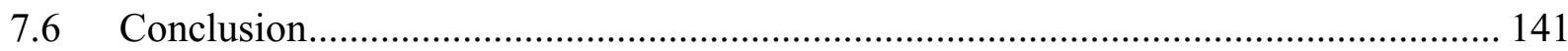

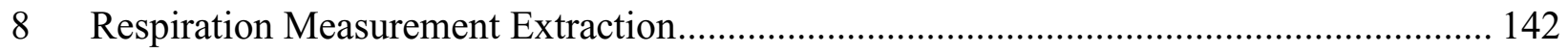

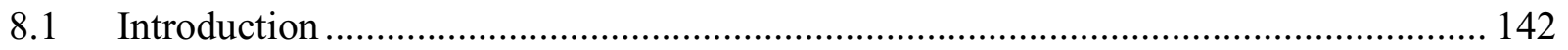

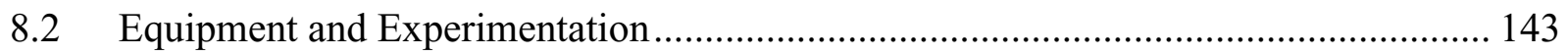

8.3 Respiration Measurement Data Analysis …………............................................... 144

8.3.1 Thermal Video Data Analysis....................................................................... 144

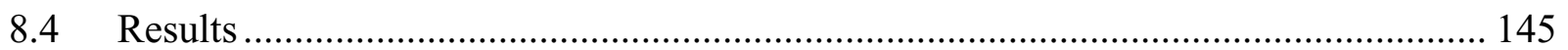

8.4.1 Normal Respiration Measurement ................................................................. 145

8.4.2 Abnormal Respiration Measurement ............................................................ 148

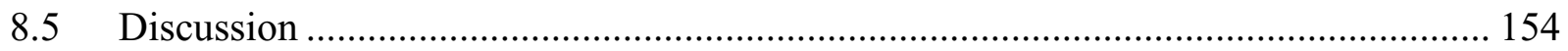

8.6 Conclusion

$9 \quad$ Temperature and Mobility Measurement Extraction ........................................................ 156

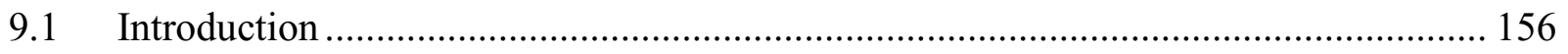

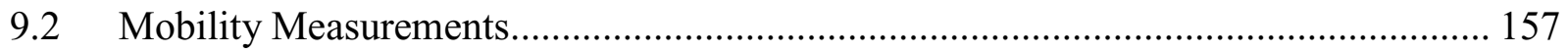

9.2.1 Equipment and Experimentation................................................................. 157

9.2.2 Mobility Measurement Data Analysis ............................................................... 159

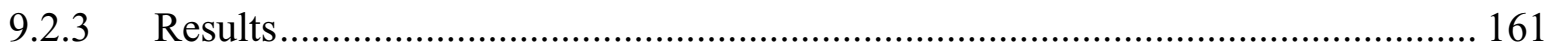

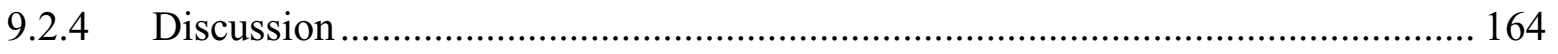

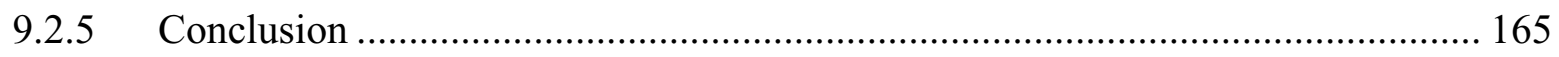




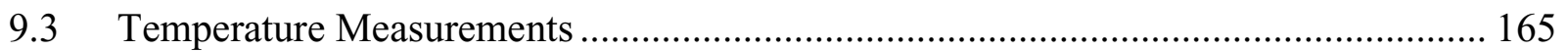

9.3.1 Equipment and Experimentation................................................................... 166

9.3.2 Temperature Measurement Data Analysis .......................................................... 167

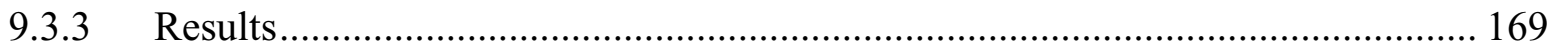

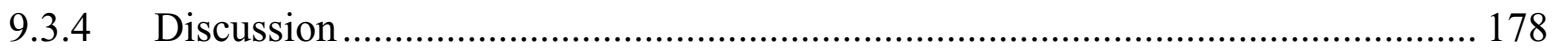

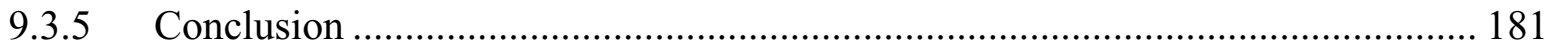

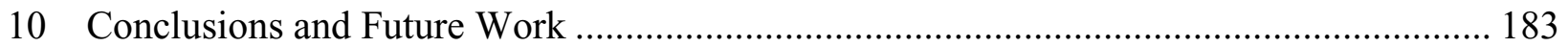

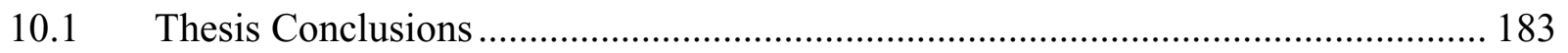

10.1.1 Data Pre-Processing and Context-Awareness....................................................... 184

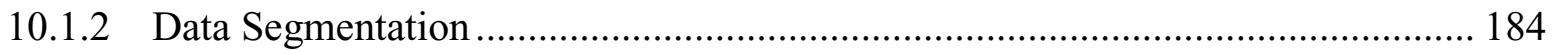

10.1.3 Video Magnification ...................................................................................... 186

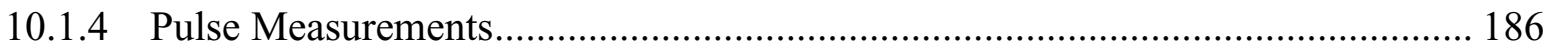

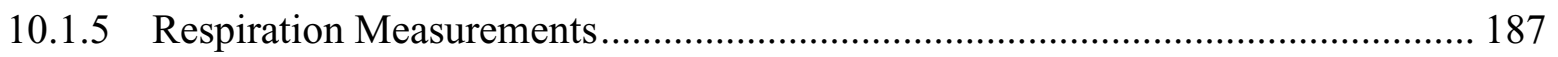

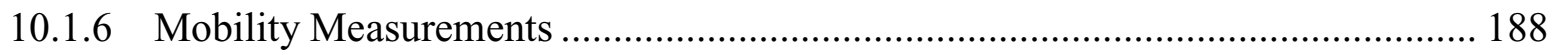

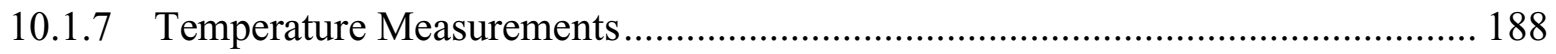

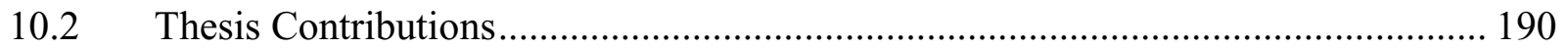

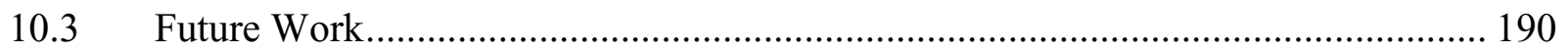

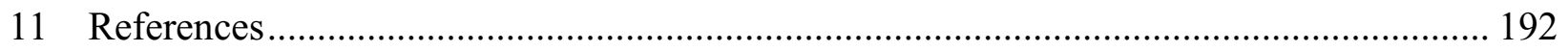




\section{List of Tables}

Table 3.1: Respiration Experimentation Subject Instructions …………………………............... 36

Table 5.1 Heel Temperature Level Pixel Areas............................................................................. 78

Table 6.1 EVM Parameter Values for Wide Passband ................................................................. 91

Table 6.2 EVM Parameter Values for Narrow Passband ………………………...................... 94

Table 6.3 Noise variance and mean square ratio ......................................................................... 99

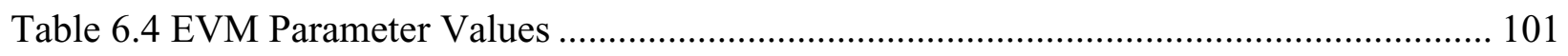

Table 7.1 Wide EVM parameter values for all thermal infrared video ..................................... 118

Table 7.2 Wide EVM parameter values for all visible light video ........................................... 120

Table 7.3 Narrow EVM parameter values for thermal video of the blood flow simulation model 125

Table 7.4 Narrow EVM parameter values for thermal video of all subjects .............................. 127

Table 8.1 Final EVM Parameter Values Normal Breathing ................................................... 147

Table 8.2 Final EVM Parameter Values Abnormal Breathing ................................................... 149

Table 9.1 Area ratios at each temperature level for both patient and healthy adult heels .......... 175

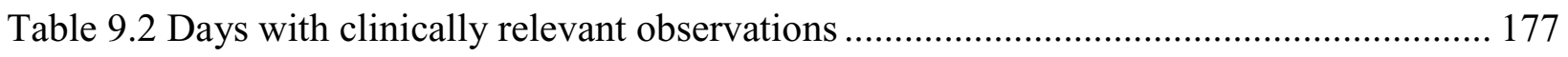




\section{List of Illustrations}

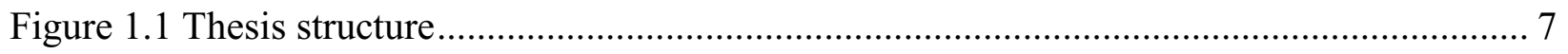

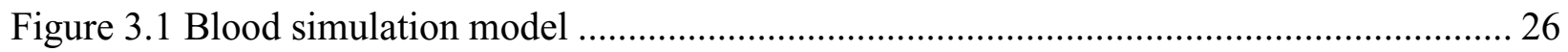

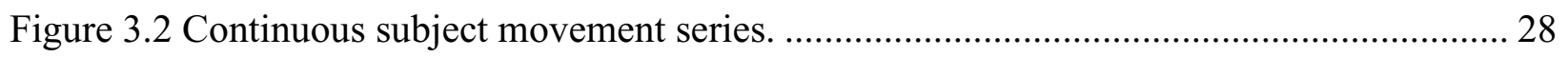

Figure 3.3 Discrete subject movement series. …………….................................................. 29

Figure 3.4 Pressure mat placement on patient beds for two and three pressure mats................... 31

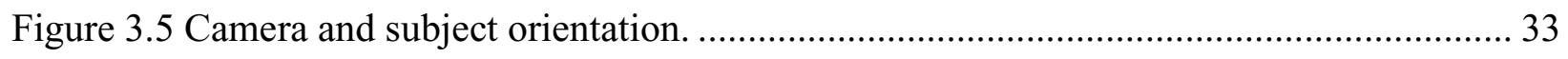

Figure 3.6 SonoScape Doppler System and study subject....................................................... 35

Figure 3.7 The Doppler Ultrasound transducer and thermal camera orientation about the subject.

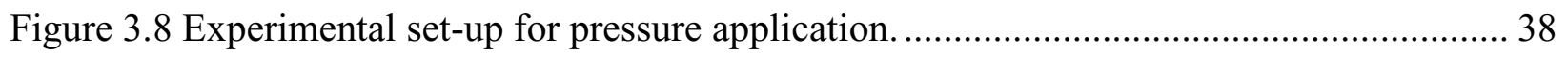

Figure 3.9 Experimental set-up for pressure application.......................................................... 39

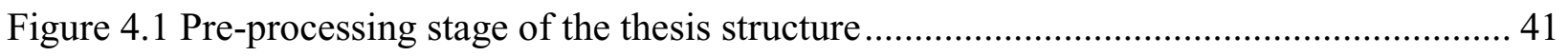

Figure 4.2 Context-awareness stage of the thesis structure ................................................... 44

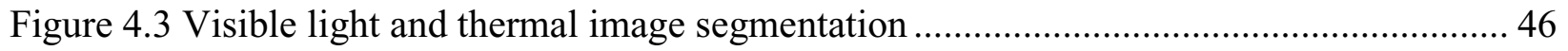

Figure 4.4 Thermal image with lights on (left) and lights off (right). ........................................ 47

Figure 4.5 Extracted pulse measurements from thermal and visible light data, as illuminance was

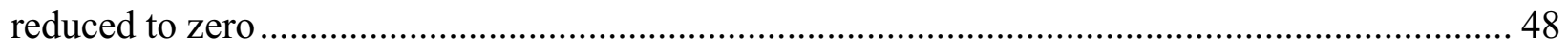

Figure 5.1 Data segmentation stage of the thesis structure .......................................................... 51

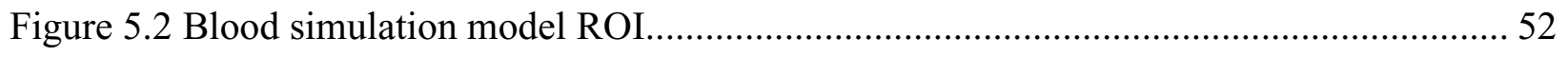

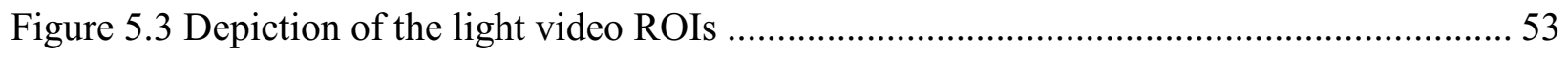


Figure 5.4 Proximal Perfusion ROIs.

Figure 5.5 The patient's asymmetric temperature distribution of the heels (top plots) versus the healthy participant's symmetric temperature distribution of the heels (bottom plots). 55

Figure 5.6 Regions of interest on the healthy adult's lateral side of the foot. 56

Figure 5.7 Regions of interest on the patient's heels. ........................................................ 56

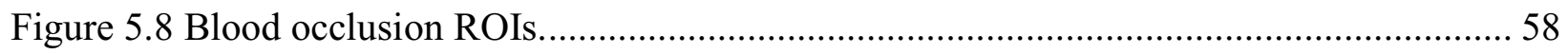

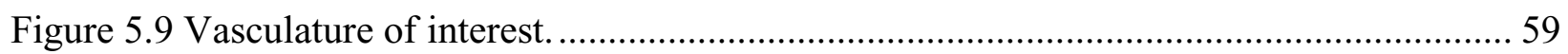

Figure 5.10 The segmentation results at 40 seconds. This was a time at which the cuff had not inflated. The vein is visible and blood flow through the artery is normal. 61

Figure 5.11 The segmentation results at 90 seconds. At this time, the cuff was fully inflated. The vein is still visible, artery is no longer receiving blood. 61

Figure 5.12 The segmentation results at 110 seconds. The blood pressure the cuff had stopped operating and was deflated. The vein remains open and blood is flowing through the artery again.

Figure 5.13 The vasculature of the forearm. Image from [125]. 63

Figure 5.14 A subject's brachial arterial diameter in response to an operating blood pressure cuff as measured by a HDI 5000 Ultrasound Instrument, where these images were quantified using

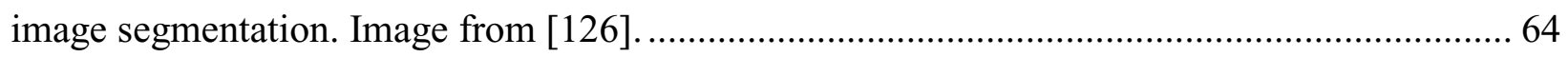

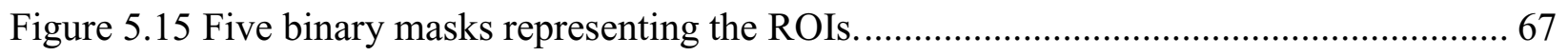

Figure 5.16 Image subjected to edge detection and thresholding, and morphological opening... 68

Figure 5.17 Image subjected to connected components labeling and region identification. 69

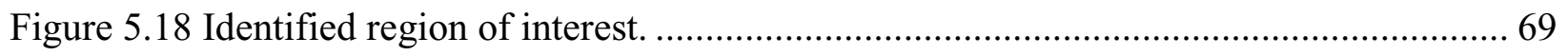

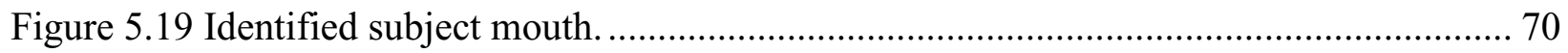


Figure 5.20 Depicted method of locating a subject's nostrils. 71

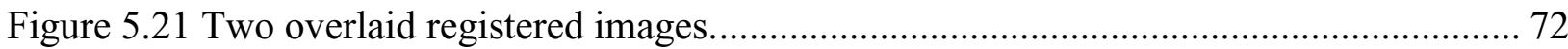

Figure 5.22 Contour plot at specified levels.............................................................. 73

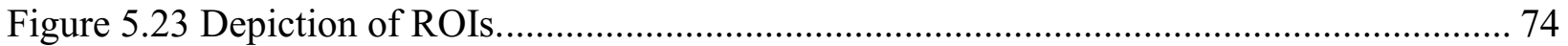

Figure 5.24 Contour plots of the baseline and three recovery images following the application of

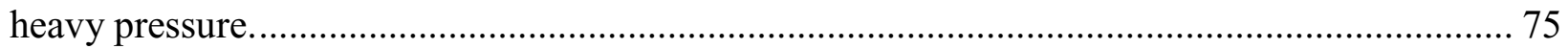

Figure 5.25 Grayscale image of the left heel and the resulting contour plot........................... 76

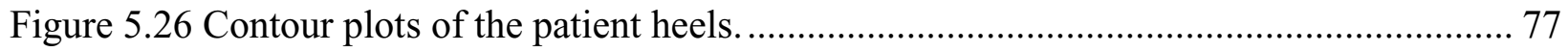

Figure 5.27 Contour plots of a healthy adult's heels. ........................................................ 78

Figure 5.28 Regions of interest from each anatomical position. .......................................... 80

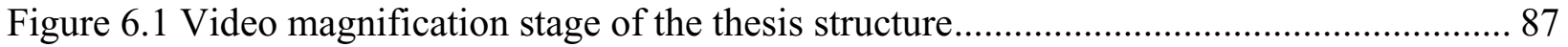

Figure 6.2 Eulerian video processing steps (image from [9])......................................... 88

Figure 6.3 Extracted vital signals from visible light and thermal infrared video (EVM bandpass

filter $1.33 \mathrm{~Hz}$ to $1.5 \mathrm{~Hz}$ ) versus ECG signal. ................................................................... 89

Figure 6.4 Light video ROI mean intensity signal (EVM bandpass filter 0.5 to $1.5 \mathrm{~Hz}$ ) versus ECG

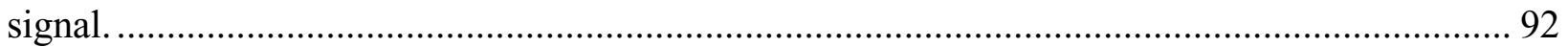

Figure 6.5 Light video ROI mean intensity signal versus thermal video ROI mean intensity signal

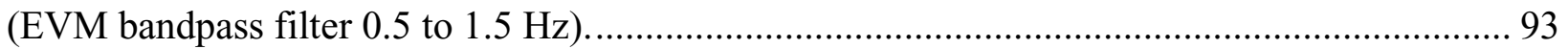

Figure 6.6 Thermal video ROI mean intensity signal (EVM bandpass filter 0.75 to $1 \mathrm{~Hz}$ ) versus

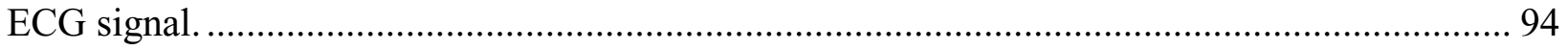

Figure 6.7 Depiction of the area of interest's sinusoidal changes. ......................................... 97

Figure 6.8 Region of interest: the simulated subject's forehead ...................................... 100

Figure 6.9 Region of interest: the subject's forehead …................................................. 100 
Figure 6.10 Original and filtered heart rate signals extracted from a subject, processed with two separate bandpass filters 103

Figure 6.11 Original and filtered heart rate signals at a frequency of $60 \mathrm{bpm}$, MSR of 5, subjected to two separate bandpass filters 104

Figure 6.12 Cumulative results, noise level 1 105

Figure 6.13 Mean intensity signal, pre-filtering for noise level level 1. 106

Figure 6.14 Cumulative results, noise level 9 107

Figure 6.15 Mean intensity signal, pre-filtering for noise level 9 107

Figure 6.16 Cumulative results, noise level 13 108

Figure 6.17 Mean intensity signal, pre-filtering for noise level 13 108

Figure 6.18 Noise levels and respective MSR values 109

Figure 6.19 AEVM applied to videos subjected to noise level 9. 110

Figure 6.20 AEVM applied to videos subjected to noise level 9 with adjusted y axis. 111

Figure 7.1 Algorithmic system with highlighted chapter focus 116

Figure 7.2 Blood simulation model ROI. 123

Figure 7.3 Wide, Eulerian enhanced optical mean intensity signal. 124

Figure 7.4 Wide, Eulerian enhanced thermal mean intensity signal.

Figure 7.5 Power spectral density plot of the wide temporal Eulerian enhanced thermal mean intensity signal. 125

Figure 7.6 Narrow temporal Eulerian enhanced thermal mean intensity signal verses pump flow rate. 126

Figure 7.7 Subject 1 Eulerian enhanced mean intensity signals versus heart rate. 128

Figure 7.8 Subject 2 Eulerian enhanced mean intensity signals versus heart rate. 128 
Figure 7.9 Subject 3 Eulerian enhanced mean intensity signals versus heart rate.

Figure 7.10 Subject 4 Eulerian enhanced mean intensity signals versus heart rate. 129

Figure 7.11 Subject 5 Eulerian enhanced mean intensity signals versus heart rate. 130

Figure 7.12 Regions of interest on the healthy adult's lateral side of the foot. 131

Figure 7.13 Healthy adult region 1 mean intensity signal plotted against the Doppler signal. .. 131

Figure 7.14 Regions of interest on the patient's heels. 132

Figure 7.15 Patient's right medial malleolar region plotted against the left. 132

Figure 7.16 Temperature changes at the femoral and wrist regions because of blood occlusion.

Figure 7.17 Frame 1 original thermal image. 135

Figure 7.18 Frame 1 cropped and segmented image using basic thresholding. 135

Figure 7.19 Object area percentage measurement over time, extracted via basic thresholding, DRLSE and watershed methods. 136

Figure 8.1 Algorithmic system with highlighted chapter focus 143

Figure 8.2. Absolute difference image. 145

Figure 8.3. Three different thermal video frames (top subplot) and the same video frames after Eulerian video magnification (bottom subplot). 146

Figure 8.4. Normal respiration intensity values plotted with RIP signals. 148

Figure 8.5. Hyperventilation intensity values plotted with RIP signals. 149

Figure 8.6. OSA simulation intensity values plotted with RIP signals. 150

Figure 8.7. Breathing rate as detected by the chest strap (true biological signal) and the calculated Method 1 ROI mean intensity signal. 151 
Figure 8.8. Breathing rate as detected by the chest strap (true biological signal) and the calculated Method 2 ROI mean intensity signal 152

Figure 8.9. Breathing rate as detected by the chest strap (true biological signal) and a thermalbased measurement for the hyperventilation test. 152

Figure 8.10 . Breathing rate as detected by the chest strap (true biological signal) and the motionbased measurement. 153

Figure 8.11 . Breathing rate as detected by the chest strap (true biological signal) and the motionbased measurement for the hyperventilation test. 154

Figure 9.1 Algorithmic system with highlighted chapter focus 157

Figure 9.2 Mobility subject movement series...... 158

Figure 9.3 COP signals for a lie-to-sit-to-stand movement. 162

Figure 9.4 COP signals for lie-to-lie, lie-to-sit and sit-to-stand transitions. 162

Figure 9.5. Time averaged, filtered sensor values and standard deviations over the duration of one night. 163

Figure 9.6. Contour plot at specified levels. 167

Figure 9.7. Depiction of the ROIs 168

Figure 9.8 Changes in temperature areas due to light pressure over time. 170

Figure 9.9 Changes in temperature areas due to heavy pressure over time. 170

Figure 9.10 ROI specific mean intensities due to light pressure over time. 171

Figure 9.11 ROI specific mean intensities due to heavy pressure over time. 172

Figure 9.12 ROI specific weighted centroids due to light pressure over time. 172

Figure 9.13 ROI specific weighted centriods due to heavy pressure over time. 173

Figure 9.14 Contour plots of the patient heels. 174 
Figure 9.15 Contour plots of the patient heels.

Figure 9.16 Left heel temperature compared to right heel temperature.

176

Figure 9.17 Temperature difference over time between the right and left heel. 


\section{Chapter 1}

\section{Introduction}

This chapter details the motivation for this thesis, reviews the problem statement and objectives as well as states contributions and describes the thesis structure.

\subsection{Motivation}

As the world's population both expands and ages, healthcare organizations fight to adapt [1]. The financial strain accompanying these population changes exasperates already understaffed hospitals and overflowing patient volumes [1]. As a result, there is a large market for inexpensive, unobtrusive, automated healthcare solutions for incorporation into hospital environments or home environments for at-home health. These solutions are often sensor based monitoring systems providing continuous patient monitoring and automated patient assessments. There is a need for these monitoring systems to be inexpensive, compact and multi-functioning; providing measurement of several biological signals with minimal technology.

\subsection{Problem Statement}

A substantial portion of routine care requires contact with a patient. In some instances, this is important and in others it is unideal, for example in mobility impaired, frail patients or in remote patient monitoring. As a result, there has been a lot of recent work completed towards non-contact monitoring of in-bed patient health, particularly in monitoring mobility and vital signs [2]. Vital signs are set of commonly measured signals used internationally as a baseline in medicine and 
surgery [3]. Vital signs are the common denominator for most acute illnesses and many chronic diseases; consistent and accurate monitoring can lead to faster intervention and better patient outcomes. Vital signals include, heart rate (HR), respiratory rate (RR), body temperature $(T)$ and blood pressure (BP) [3]. When considering in-bed patients a fifth vital sign should be considered; mobility, which is particularly relevant for older adult in-bed patients with multiple co-morbid conditions [4]. Despite the clear clinical importance of vital sign measurements, there is often missed or inadequate documentation of patient vital sign measurements by healthcare workers and clinical deterioration is detected too late or not at all [5]. A study in Thunder Bay, Ontario, Canada showed that on admission to hospital, all vital signs were measured from $99.6 \%$ of 18,853 acutely ill patients [6],[7]. While the hospital's policy was to record vital sign measurements from each patient every six hours, only $82 \%$ had a documented second set of vital sign measurements and only $66 \%$ had a documented third set of vital sign measurements [6],[7]. The development of unobtrusive, automated and continuous monitoring offers the potential to enhance the safety and quality of patient care [8].

Many sensor types can be used unobtrusively to extract vital health information [2]. Video cameras, for example, are generally inexpensive, low risk, unobtrusive and capable of capturing more than one vital signal as well as being able to distinguish between more than one subject [2]. Thermal cameras can capture the same vital signals as visible light cameras and additionally, can capture subject data in the absence of light and intrinsically measures one vital sign that visible light cameras do not: temperature [2]. While these measures are important, sensors that are unable to capture all five vital signs individually are insufficient for vital sign monitoring on their own. Systems containing multiple sensors measuring different physiological phenomena provide more statistically independent information and better error estimation [2]. This thesis develops a system 
that utilizes pressure mat data, visible light video and thermal infrared video to capture all vital signals of interest.

A common and effective method of extracting vital signal measurements from subject video data is through Eulerian Video Magnification (EVM); a spatio-temporal video processing method developed at MIT, which enhances temporal color or motion patterns in videos such as the color changes in a face due to pulse rate and blood perfusion, or the rise and fall of the lungs breathing [9]. While the use of EVM has been shown to successfully and unobtrusively enhance biological signals, the drawback of the algorithm is that that it can significantly amplify noise as the magnification factor increases. This fact, coupled with the fact that the algorithm parameters are user-defined can lead to the amplification of noise alone and the false attribution of this signal to physiological phenomenon (vital signal measurement) [10],[11]. Furthermore, EVM is most commonly applied to visible light video; more research is required in the application of EVM algorithms to thermal video data of subjects and the extraction of vital signal measurements.

\subsection{Objectives}

The main objective of this thesis is to examine, test and improve upon the current EVM processing technique and expand it's use to thermal video vital signal extraction. This thesis does this within a system framework that is designed to extract vital signal measurements from in-bed subjects using three modalities in conjunction with image and signal processing. The three modalities include thermal infrared cameras, visible light cameras and pressure sensitive mats. Vital signals include pulse, respiration, temperature and mobility. Healthy adult and older adult patient subjects participated in several experimental procedures including thermal and visible light camera data capture of faces, hands and feet as well as in-bed pressure mat data capture. A system of algorithms 
was developed for this thesis to automatically extract vital sign measurements from a subject. This thesis aims to not only extract vital signal measurements, but explore patterns associated with inbed, older adult morbidities, and the ability of the proposed system to detect these abnormalities. While this thesis examines both older adult in-hospital subjects and healthy adult volunteers, the target population are those that require long term health monitoring.

\subsection{Summary of Contributions}

This thesis contains several pillars of research. The contributions presented in this section are representative of these pillars and include:

1. The first contribution was in the examination of center of pressure (COP) as it relates to mobility measures. Continuous pressure mat data was captured of healthy adult subjects as they transitioned between several orientations on a hospital bed. The COP was extracted, as well as the COP velocity and acceleration in the $\mathrm{x}$ and $\mathrm{y}$ directions. Cumulatively, the COP data was able to characterize each transition, indicating the value of the COP in mobility monitoring.

[12] S. Bennett, Z. Ren, R. Goubran, K. Rockwood, and F. Knoefel, "In-Bed Mobility Monitoring Using Pressure Sensors," in IEEE Trans. Instrum. Meas., vol. 64, no. 8, May 2015, pp. 2110-2120.

2. The second contribution was in image segmentation. For the extraction of any vital signal from an image or video, the correct anatomical region of interest needs to be identified. Extensive work was completed on image segmentation of various body parts, including techniques such as level set method, watershed method and region tracking for vital signal measurements. 
[13] S. Bennett, R. Goubran, and F. Knoefel, "Comparison of Motion-Based Analysis to Thermal-Based Analysis of Thermal Video in the Extraction of Respiration Patterns," in Proc. IEEE Int. Conf. Eng. Med. Biol. Soc. (EMBC), Jeju Island, South Korea, July 2017, pp. 1-5.

[14] S. L. Bennett, R. Goubran, and F. Knoefel, "Examining Changes in Image Segmented Arteries in Response to Blood Occlusion," in Proc. IEEE Med. Meas. Appl. (MeMeA), Rochester, Minnesota, USA, May 2017, pp. 1-5.

3. The third contribution was the development and analysis of the adaptive Eulerian video magnification (AEVM) method, which is used throughout this thesis. The algorithms modify an existing method (EVM), by adding an iterative step in which a passband filter's cut-off frequencies are optimized. This method was used to extract pulse rate measurements from thermal video; a modality not thoroughly explored in the context of EVM. Data collection for this contribution involved data capture from healthy adult subjects, older adult patient subjects, and a mechanical system built to simulate the pulsatile nature of a heart and the consequent flow of liquid.

[15] S. L. Bennett, R. Goubran, and F. Knoefel, "Examining the Effect of Noise on Biosignal Estimates Extracted through Spatio-Temporal Video Processing," accepted to Proc. IEEE Int. Conf. Eng. Med. Biol. Soc. (EMBC), Berlin, Germany, June 2019, pp. 1-5.

[16] S. L. Bennett, R. Goubran, and F. Knoefel, "Context-awareness in non-contact, multimodality, bed-based monitoring of vital signs," in Proc. IEEE Med. Meas. Appl. (MeMeA), Rome, Italy, June 2018, pp. 1-5 
[17] X. He et. al., "Video-based analysis of heart rate applied to falls," in Proc. IEEE Med. Meas. Appl. (MeMeA), Rome, Italy, June 2018, pp. 1-5.

[18] S. Bennett, T. N. El Harake, R. Goubran, and F. Knoefel, "Adaptive Eulerian Video Processing of Thermal Video: An Experimental Analysis," in IEEE Trans. Instrum. Meas., April 2017, pp. 1-9.

[19] S. L. Bennett, R. Goubran, B. Bennett, R. Bennett, and F. Knoefel, "The Use of a Thermal Camera and Eulerian Enhancement in the Examination of Pedal Pulse and Microvascular Health," in Proc. IEEE Int. Conf. Eng. Med. Biol. Soc. (EMBC), Orlando, Florida, August 2016, pp. 1-4.

[20] S. L. Bennett, R. Goubran, and F. Knoefel, “Adaptive Eulerian Video Magnification Methods to Extract Heart Rate From Thermal Video," in Proc. IEEE Med. Meas. Appl. (MeMeA), Benevento, Italy, May 2016, pp. 1-5.

[21] S. L. Bennett, R. Goubran, and F. Knoefel, "The Detection of Breathing Behavior Using Eulerian-Enhanced Thermal Video," in Proc. IEEE Int. Conf. Eng. Med. Biol. Soc. (EMBC), Milan, Italy, August 2015, pp. 7474-7477.

4. The fourth contribution was the examination of temperature and mobility measurements from in-bed subjects independently, and in relation to each other. This contribution is valuable, as the data examined originates from older adult in-hospital patients and therefore speaks directly to morbidities related to this co-hort.

[22] S. Bennett, R. Goubran, and F. Knoefel, "Long Term Monitoring of a Pressure Ulcer Risk Patient Using Thermal Images," in Proc. IEEE Int. Conf. Eng. Med. Biol. Soc. (EMBC), Jeju Island, South Korea, July 2017, pp. 1-4. 
[23] S. L. Bennett, R. Goubran, and F. Knoefel, "Measurement and Comparison of Thermal and In-Bed Pressure Data towards the Prevention Pressure Ulcers," in Proc. IEEE Int. Conf. Biomed. Health Informatics (BHI), Las Vegas, USA, February 2016, pp. 62-65.

[24] S. L. Bennett, R. Goubran, and F. Knoefel, "Measurements of Change in Thermal Images Due to Applied Pressure," in Proc. IEEE Med. Meas. Appl. (MeMeA), Turin, Italy, May 2015, pp. 30-35.

\subsection{Thesis Structure}

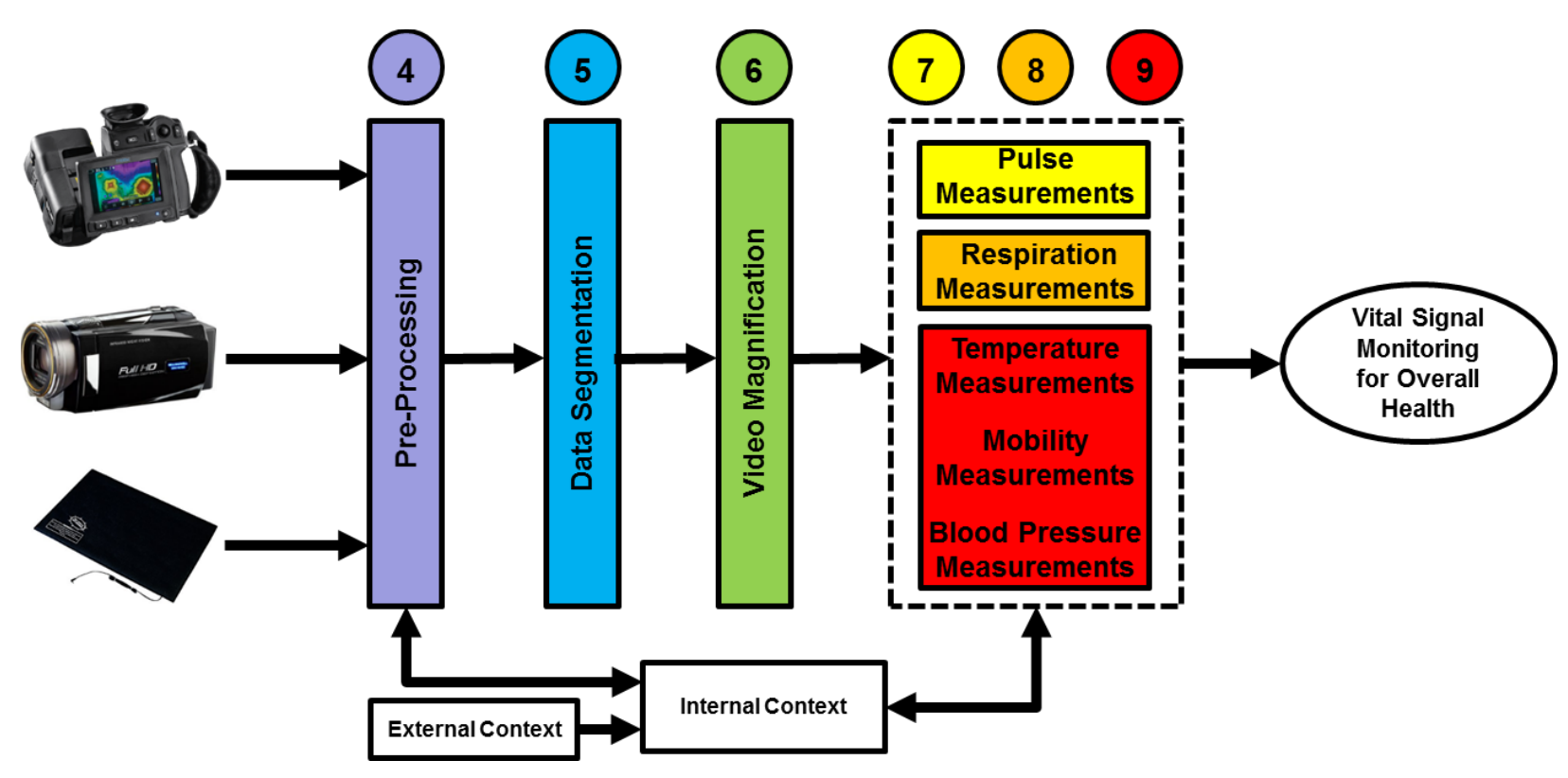

Figure 1.1 Thesis structure

The structure of this thesis is depicted in Figure 1.1, and is organized into ten total chapters. A detailed background on the modalities used in the extraction of vital signals, examined in this thesis is given in Chapter 2. Chapter 2 is followed by a description of the equipment set-up, experimental procedures and experimental conditions used to generate and gather data in Chapter 3. Chapter 4 
describes the overall system set-up, the pre-processing stage and the role of context-awareness within the system. The second stage of the system, region identification and image segmentation, is described in Chapter 5. Chapter 6 details the EVM method, and the developed adaptive EVM method; the primary contribution to this thesis. Chapters 7-9 detail the pulse, respiration, temperature and mobility vital signal extraction methods, respectively. Chapter 10 discusses and concludes the research described in this thesis. 


\section{Chapter 2}

\section{Background}

This chapter details background information, which explains the motives behind this thesis project and the previous work upon which this project is built.

\subsection{Introduction}

This thesis uses three different modalities to extract four vital signal measurements from subjects; pressure sensitive mats, visible light cameras, and thermal cameras to extract pulse, respiration, mobility and temperature measurements. This chapter reviews background information relevant to this thesis. Section 2.2 discusses the use of the modalities of interest in the extraction of vital signals to date. Sections 2.3 and 2.4 discuss image segmentation and Eulerian Video Magnification (EVM) as they apply to vital signal measurement. Section 2.5 details each of the four vital signals examined in this thesis, and reviews current methods of extracting these signals.

\subsection{Modalities}

Recent advancements in non-contact monitoring of respiration and pulse measurements include those in radar, visible light video and thermal video. Radar has been shown to successfully detect heart rate and respiration rate [25]-[27]. However, the technology measures cardiorespiratory signals through movement only and is therefore limited in the amount of patient information it can convey [28]. A visual image representation of a patient conveys more information through one source; in addition to heart rate and respiration rate, cameras can more easily detect and monitor 
multiple subjects and have even been shown to capture subject emotion (which is helpful in monitoring dementia patients) [29], [30].

Visible light video has been shown to reveal heart rate and respiration rate [30],[31]. Common methods include motion analysis for respiration rate [32], and color analysis for heart rate [33]. While visible-light video has successfully extracted vital signals, it is highly susceptible to changes in lighting and skin color [2].

Thermal video has also been shown to reveal heart rate and respiration rate, while being impervious to changes in lighting and skin color [34],[35]. Additionally, thermal video can monitor a subject's temperature; a vital sign that neither radar nor visible light cameras are able to monitor [2]. Heart and respiration rate is extracted from thermal video by evaluating motion or temperature changes over time [36],[37]. While vital signals have been extracted from thermal camera, less peer-reviewed research has been published in this area and the resulting vital signal measurements are not as strongly corroborated with a body of work.

Patient mobility has been examined using several different sensing methods; accelerometry and pressure sensing are among the most widely examined and are emerging fields [38]. Accelerometry requires that a patient wear an accelerometer device, which is undesirable for a frail patient in critical condition. Pressure sensors on the other hand, do not come in contact with the patient at all and have a very fine sensitivity, enabling detection of patient rollovers at night, detection of movement onset times (movement initiation time), and even collection of respiratory rate information [39]-[41]. Of importance to biomedical applications are pressure sensing methods using optical fibers [42]. Silica fibers in biomedical applications can be thinner than a standard surgical suture and can be used in a clinical setting without any electrical or chemical interference with existing technical set-ups [42]. Fiber optic pressure mats are intrinsically safe, have a high 
sensitivity, can be small, of low cost and perform well over time (continuous monitoring) [43]. Work completed using fiber optic pressure sensor arrays, particular to older adult patient mobility monitoring is inclusive of identification and distinction between several different in-bed orientations, and transitions between orientations.

\subsection{Image Segmentation}

Image segmentation is a widely researched topic in biomedical image processing; particularly in the realm of region of interest (ROI) identification and ROI tracking. Segmentation in this thesis ranges from region identification using binary masks, to region detection and tracking using more complicated algorithms. While the creation of new segmentation techniques is not the goal of this thesis, the use of existing techniques on different data types as a stage in the extraction of vital signals from in-bed subjects is one of the contributions of this thesis. Specifically, this thesis examines the application of visible-light image segmentation techniques to thermal-infrared images of vasculature; an area more recently explored, and to a lesser extent than visible light images.

Thermal images have been used in the identification of facial vasculature, identifying tissue boundaries in surgery [44]-[46]. While vasculature has been examined using thermal images, it has most often been examined in relation to vein pattern biometrics [47]-[49], as opposed to the assessment of blood flow. The studies that have used thermal imaging to examine blood flow have often calculated average temperature from a region of interest over time [50],[51], as opposed to segmenting and examining the behavior of specific veins. The aversion to advanced image segmentation methods is likely due to the nature of thermal images; characteristically low contrast, low detail, highly variant images. 
While there are comparatively few thermal imaging studies segmenting blood vessels to examine blood flow, there are many studies segmenting retinal blood vessels from retinal images (visible light images), which are similarly shaped objects from a different imaging modality. There are a wide range of segmentation methods used, including ridge detection and morphological based segmentation [52]-[54], k-means clustering and connected components analysis [55], graph-based segmentation and classification [56], watershed transform [57], level set method [58], and Convolutional Neural Networks (CNN) [59], among others.

This thesis explores the application of image segmentation methods described in this section, to thermal images in the examination of vital signal extraction.

\subsection{Eulerian Video Magnification}

The use of unobtrusive sensors to capture health information in hospital settings is becoming a widely researched area. In a time in which technology is developing far faster than hospital policy or implementation of technology, sensor solutions using equipment already present in a hospital environment or multi-functioning sensors, assessing several bio-signals at one time, are of great value. An example is the use of an automatic blood pressure cuff, a device used during routine care, to capture two biosignals; heart rate and blood pressure [60],[61]. Multi-functioning systems provide additional value when they are intuitive and can provide automatic feedback to healthcare workers. While video cameras are not currently part of hospital environments for routine care, video data in conjunction with video processing techniques is a promising future option, should privacy concerns be addressed, as it is a familiar technology that is widely available and has been shown recently to reveal vital health signals using spatio-temporal video processing techniques [9]. 
Spatio-temporal processing applied to video data to extract biosignals is an emerging technique [9]. A commonly employed spatio-temporal video processing technique is called Eulerian Video Magnification (EVM) and was developed at MIT [9]. The technique spatially decomposes a video using a Laplacian pyramid to amplify temporal variations occurring within a specified frequency band. This procedure mathematically and visually reveals hidden changes in movement or color and allows for the extraction of health signals [9]. The EVM method was designed for application to optical camera video data, and has been used to collect heart rate, breathing rate and pulse transit time, among other biosignals [9][57]-[67].

Very recently EVM has been applied to thermal camera video data [8],[21],[51],[68]. Thermal cameras have the potential to reveal biological signals that optical cameras may not be able to. For example, a subject in the dark, the effects of pressure on blood flow in the extremities, and the identification of facial features such as vasculature [43],[69],[70]. Thermal video in conjunction with EVM has been shown to identify respiration rates and respiration patterns indicative of central and obstructive sleep apnea, and has been used to measure arterial pulse at the feet [19],[21]. The EVM method has also been applied to thermal video of an adult's face to reveal heart rate [20],[68].

The EVM method has been shown to extract vital health information from thermal video, and is a possible solution to the issues of subject skin color and ambient lighting conditions encountered in Eulerian enhancement of optical videos [71]. However, there has not yet been enough research in the application of EVM, an existing method, to thermal video, a new modality, to confirm that the revealed temporal variations are a result of blood perfusion. This thesis thoroughly examines the EVM method as applied to thermal camera data through rigorous testing and aims to improve upon the current algorithm. 


\subsection{Vital Signals}

The four classic vital signals are pulse rate, respiratory rate, body temperature and blood pressure, which have been examined since as early as 1860 and have become part of routine care in most hospitals by 1970 [6]. Today, vital sign monitoring is one of the most common methods of tracking a patient's health improvement or decline; this is because vital signals are the simplest, most inexpensive information gathered from patients and one of the most accurate predictors of clinical deterioration [5],[6],[8],[72]. Early recognition of clinical and physiological deterioration in a subject can indicate the need for treatment and can prevent or reduce serious adverse outcomes $[6],[8],[72]$.

Despite a long history with vital sign monitoring, and the clear benefits of accurate and consistent measurements, patient deterioration is often missed by healthcare workers even though worsening vital signals often precede this deterioration [6]. Vital signal monitoring and documentation has received limited attention regarding medical research, and there exists a lack of knowledge regarding vital signal measurement optimal practices [8].

This thesis moves towards the accurate and consistent measurement of vital signals through the use of monitoring modalities, and a better understanding of vital signals as they relate to subject morbidities through the capture and examination of subject data, followed by the extraction of vital signal measurements through data processing algorithms. In addition to three classic vital signs: pulse rate, respiration rate and temperature, this thesis also examines mobility; a recognized indicator of health, particularly in older adults [73],[4]. 
The following sections review current methods of extracting vital signal measurements from the modalities of interest (thermal-infrared camera, visible-light camera, and pressure sensitive mats), and the use of these measurements in the detection of subject morbidities.

\subsubsection{Pulse Measurements}

Pulse rate is one of the vital signs examined in this thesis, and a measurement extracted routinely in hospitals to monitor patient health. This thesis examines the ability of algorithms in this thesis to assess blood flow both proximally and distally from thermal-infrared and visible-light video data.

A popular method of extracting pulse measurements from visible-light video data of subjects, is by using the EVM method. Visible-light video can be obtained easily using any number of household devices, and, in conjunction with EVM processing, has been proven to successfully extract pulse measurements from a given subject [2],[30]. However, subject skin colour and lighting conditions have been shown to have a large effect on pulse measurements. Thermal cameras are impervious to lighting conditions and subject skin colour, as well as providing temperature measurements that regular cameras may not be able to. For example, the effects of pressure on blood flow can be seen using a thermal camera but may not be revealed using regular video.

The hypothesis that EVM in conjunction with thermal video can reveal perfusion rates is supported theoretically. The heat transfer properties of blood vessels and skin, individually and combined, are not a new topic and have been modeled using the Pennes' bioheat model [74][76]. While this interaction is complex, a simple result is that the skin can change temperature periodically as a result of perfusion; blood at core body temperature is pumped to surface veins 
at a temperature closer to room temperature [68]. These temperature changes are within the mid-wave thermal camera temperature capture capabilities [43]. A highly sensitive thermal camera can identify and distinguish facial blood vessels from the skin [43]. While this work supports the thermal EVM hypothesis, more experimental research is needed.

This thesis focuses on the extraction of pulse measurements from thermal cameras. Rather than examining thermal data for pulse abnormalities and associated morbidities, this thesis examines the spatio-temporal algorithms used to extract the pulse measurements and aims to improve the algorithms, therefore improving measurement accuracy.

\subsubsection{Respiration Measurements}

Respiration rate is a vital signal examined in this thesis, is considered the most sensitive marker of a deteriorating patient and one of the first vital signals to indicate a health issue [6]. Despite this vital signal's clinical value, it is the vital sign that is least often recorded and most commonly omitted from documentation [6]. This thesis moves towards more consistent and accurate respiration measurements using thermal cameras and spatio-temporal video processing. This thesis examines the extraction of normal respiration, and respiration abnormalities. While there are many data patterns associated with different respective patient morbidities, this thesis focuses on abnormal breathing associated with sleep deficiency.

Sleep deficiency is defined as "a state of inadequate or mistimed sleep" and has been shown to cause damaging molecular changes to the immune and neural systems in the body [77]. Immune imbalances decreased antioxidant defense responses and cumulative cell stress responses in the brain may lead to the development and worsening of disease and resulting mortality [77]. Sleep deficiency is associated with a greater risk of cardiovascular disease, 
obesity, cancer and accidents due impaired cognitive or motor performances [77]. While sleep is critical for good health, an estimated 50 to 70 million adult Americans suffer from chronic sleep disorders including insomnia, narcolepsy, restless leg syndrome, REM-sleep behavior disorder and the most prevalent sleep disorder, sleep apnea [77]-[80].

Sleep apnea is a respiratory disorder and is categorized into two different types; Central Sleep Apnea (CSA) and Obstructive Sleep Apnea (OSA). CSA occurs when the brain fails to contract the diaphragm, causing the subject to stop breathing [81]. In the most common form of CSA, this occurs periodically and is followed by periods of rapid breathing [82]. OSA occurs when the upper airway narrows and collapses, while respiratory efforts continue [83].

Currently, the best method of detecting and distinguishing between OSA and CSA is polysomnography (PSG). PSG is a comprehensive physiological exam that, while effective, is expensive and invasive. Efforts have been made to capture sleep apnea through the use of unobtrusive and inexpensive devices. Force sensing resistors have been used to detect sleep parameters and sleep apnea [83], a sensor mattress has been used to collect respiratory signals and detect sleep apnea [84], an under-mattress pressure sensing grid positioned underneath a subject's chest has been used to obtain the intensity of sleep movements [85] and load cells have been used to detect periods of wakefulness [86], among other studies. While all of the described studies discuss sleep apnea and aim to contribute to the detection of sleep apnea, only some succeed in detecting the condition and none identify between OSA and CSA. There is clear need for an inexpensive, unobtrusive device capable of detecting sleep apnea and distinguishing between different apnea types.

Respiration measurements are extracted in this thesis through several stages of processing. Once extracted, the signals are examined for frequency, amplitude and temporal patterns 
indicative of regular breathing, and of abnormal breathing. The abnormal breathing examined in this thesis models that of sleep apnea.

\subsubsection{Temperature Measurements}

Temperature is another vital signal examined in this thesis, and one that is clearly measurable. This thesis therefore examines temperature as it relates to patient morbidities. Specifically, temperature is examined in relation to pressure, immobility and the development of bedsores in older adult patient data.

Temperature is extracted in thesis using thermal infrared imaging; a process by which infrared heat emissions are converted into an image indicating thermal differences [87]. Thermal imaging is used commonly in the detection and examination of inflammation leading to carcinogenesis, particularly in breasts [88],[89]. It has been used to examine vascular properties such as perfusion [90], dermatological properties such as skin temperature and symmetry [91]-[93] and in orthopaedic applications such as the examination of bone healing [94]. Thermal imaging has also been used in the examination of pressure ulcers. Infrared thermal images in conjunction with digital light images have been used to indicate a developed Stage 1 pressure ulcer [95], as well as to examine the healing process of a pressure ulcer [96]. Thermal images have been used to examine temperature changes indicating increased or decreased blood flow in diabetic subjects' feet [97]-[99]. While thermal imaging has been successfully used in the examination of existing ulcers and the healing process after ulcer formation, there have not been many applicable results regarding prevention of pressure ulcers [98]. Considering the nature of pressure ulcer formation (pressure results in a lack of blood 
flow which causes tissue death), it seems likely that a pattern in temperature may exist for the formation of a pressure ulcer allowing for early detection and intervention.

This thesis examines temperature as it relates to blood flow and pressure; aiming to establish a pattern in this relationship. Older adult in-hospital data were examined over several months to characterize the formation of pressure ulcers and move towards prevention, however no patient fully developed a pressure ulcer, and so this thesis presents more observational than conclusive pressure ulcer formation results.

\subsubsection{Mobility Measurements}

The last vital sign examined in this thesis is mobility. This thesis builds upon previous work focused on the assessment of in-bed mobility. The positional lying state and associated transitions have been examined through the use of regions of interest and ratio calculations to identify incidences of pressure point relief [100],[101]. The positional sitting state and associated transitions have been examined using classifiers [102]. Specifically, one body of work aimed to determine first if a subject was sitting or lying in one of several orientations, and then if a subject was transitioning from lying to sitting while in bed [102],[103]. The body of work detailed the examination of support vector machines (SVMs), nearest neighbor (NN) and k-nearest neighbor $(\mathrm{kNN})$ classifiers in the determination of one final stationary classifier (SVM) that would distinguish between four different lying positions and two different sitting positions [102],[103]. A separate classifier was found to distinguish between the state transitions. The positional sitting and standing states (and associated transitions) have been examined in terms of timing and bouncing [104],[105]. Pressure images were used to identify regions of interest, assess the symmetry of transfers and detect bouncing [104]. Movement 
trajectories and duration measurements were calculated and used to detect mobility impairment and distinguish from the un-impaired [105].

While this work is important in moving towards long term patient mobility monitoring, the methods are thus far unrealistic; all of the described studies use discrete data sets generated by healthy adult volunteers. In-hospital patient monitoring consisting of continuous data, multiple positional states and state orientations is important in developing algorithms that will accurately assess mobility of an older adult patient as well as a healthy young adult. This thesis therefore utilizes fiber optic pressure sensor arrays and builds upon previous work specific to patient mobility to develop algorithms capable of identifying and distinguishing between all of the states and state transitions.

In addition, this thesis examines the Center of Pressure (COP). COP has been shown to be an advantageous measure in gaining insight into human movements. The measure has been used to examine pressure points underneath a seated subject [106] and to analyze the stability of human gait [107] among other applications. Specific to the movements of interest, COP has been calculated, descriptive features extracted from the signal and used in the linear and SVM classification of a stable sit-to-stand transfer verses an unstable sit-to-stand transfer [108]. COP magnitude and frequency has also been captured for a continuous lie-to-sit-to-stand bed exit performed by both an older adult patient and a young healthy volunteer to visually identify differences between bed exit COP signals [109]. 


\section{Chapter 3}

\section{Experimental Conditions and Procedures}

This chapter details the ethics, equipment, experimental conditions and experimental procedures used for experimentation with humans and simulations.

\subsection{Introduction}

This chapter details the ethics clearances required for experimentation, the technical specifications of the three modalities used for this thesis, amongst other auxillary equipment. This chapter then details the experimental conditions and procedures for this thesis, which involves human experimentation as well as experimentation with a mechanical simulation system. For human experimentation, data was captured of healthy adults in controlled environments, as well as of older adult patients in hospital settings. The mechanical simulation system was examined in a controlled environment. The following chapter is organized by topic and discussed in detail.

\subsection{Ethics}

For all human experimentation, standard university ethics procedures were completed, and approval was obtained. This included experimentation with 14 volunteers and 8 older adult patients at risk of developing pressure ulcers. Sample size is discussed in the following sections. For all older adult patients studied, Bruyère continuing care ethics procedures were completed and approval was obtained. In accordance with ethics procedures, all subjects provided consent to gather data and present results. No other health information is known about the patients, as ethics 
clearance did not include access to patient health records. Patients were determined to be at risk of developing pressure ulcers by means of the Braden Scale [110]; due to the health record access limitations, researchers were not informed of the patient's Braden score, but were informed that a patient had been identified for the study. While data was initially gathered from patients to study the development of pressure ulcers, no pressure ulcers officially formed, so the results pertaining to the examination of pressure ulcer development and prevention are suggestive rather than conclusive.

\subsection{Equipment}

This thesis utilizes three modalities to extract health information; thermal cameras, a visible light camera, and pressure mats. The technical specifications for these modalities are detailed in this section, as well as the specifications for all other equipment used in experimentation.

\subsubsection{Human Trials}

Human experimentation utilized several types of equipment, inclusive of pressure sensitive mats, three different thermal cameras, an HD digital camera, a wireless blood pressure cuff, a pulse oximeter, a Doppler ultrasound system, a chest strap measuring physiological signals, a laptop and a thermometer.

The pressure sensitive mat was manufactured by S4 Sensors and contains a 3 by 8 pressure sensor array. Each sensor is comprised of two optical fibers and an integrating cavity, or a scattering medium. Light from an LED travels through one optical fiber into the integrating cavity (the scattering medium is polymer foam) and travels back through the other optical fiber to a photodiode. Applied pressure scatters the light in the integrating cavity, which affects the light traveling to the photodiode. The photodiode measures the change in light and outputs a 
voltage proportional to the pressure applied. Associated S4 Sensors software transforms the output voltages to nominal scores proportional to the pressure applied.

The three thermal cameras were all mid-range infrared cameras, but varied in resolution, sensitivity and ease of use. The FLIR E4 infrared camera is the easiest to use as it is handheld. The infrared camera has a thermal sensitivity of $<0.15^{\circ} \mathrm{C}$, a temperature range of $-20^{\circ} \mathrm{C}$ to $250^{\circ} \mathrm{C}$ and an accuracy of $\pm 2 \%$. It can take thermal images as well as light images and the associated FLIR software allows the user to save image types separately or as superimposed images on a laptop. The more sensitive, but less versatile thermal camera is the mid-range FLIR A-Series infrared (IR) camera that captures temperature within the range of $-20.0^{\circ} \mathrm{C}$ to $350.0^{\circ} \mathrm{C}$ with an accuracy of $\pm 2 \%$ and sensitivity of $<0.05^{\circ} \mathrm{C}$. The IR camera has a resolution of $320 \times 240$ and captures thermal video (at $60 \mathrm{~Hz}$ ) as well as thermal images. The most sensitive camera used for experimentation is the FLIR T1030sc infrared (IR) camera, which has an accuracy of $\pm 1 \%$, sensitivity of $<0.02^{\circ} \mathrm{C}$, a resolution of $1024 \times 768$, and captures thermal video at $13.5 \mathrm{~Hz}$. Thermal camera accuracy is a complicated measurement that is discussed in more detail in [111]. Full technical specification sheets can be found for all three thermal cameras in [112]-[114].

The HD digital camera, manufactured by Bell \& Howell has a resolution of 1920 x 1080p, a frame rate of $30 \mathrm{~Hz}$ and 10x optical zoom. The digital camera video is referred to in this thesis as visible light video to distinguish between digital and thermal video.

The Doppler ultrasound system is manufactured by SonoScape is a S2 Portable Digital Color Model with a linear transducer. The transducer has a scan range of $\geq 132^{\circ}$ and a frame rate of $>137 \mathrm{~Hz}$. The Doppler ultrasound was used to quantify blood flow at the extremities. 
The wireless blood pressure cuff is manufactured by Quardio and was placed on the arm of the subject to occlude and measure blood flow. The cuff is controlled wirelessly via an application on a mobile device; inflating to get the systolic blood pressure and deflating to get the diastolic blood pressures.

The pulse oximeter was manufactured by Contec Med and displays $\mathrm{SpO} 2$ as well as the pulse rate and pulse waveform but does not transmit data wirelessly. When pulse oximeter measurements are required over time, the pulse oximeter display screen was captured by the digital camera simultaneously. The pulse oximeter data was obtained by a researcher later reviewing the video data and manually recording the pulse oximeter data at specific times, or as an average over time.

The Zephyr chest strap captures heart rate and breathing rate, among several other physiological signals not examined in this thesis. The device is used for validation purposes, is worn by the user for the duration of data collection and removed afterwards for data download.

The Hexoskin smart shirt is a garment containing various textile sensors for capturing biological information. This thesis uses only the ECG sensors, which gather data at $256 \mathrm{~Hz}$. It is connected to a small data bus that both gathers and transmits this information to a smart device. The Hexoskin application was downloaded onto a smart phone, which was used to control the smart shirt.

The laptop was used to gather data from the pressure mat via Bluetooth as well as to save uploaded thermal images. The Wavetex Meterman thermometer was used to measure ambient temperatures for validation purposes. 


\subsubsection{Simulation}

The simulation experiments involved the design and development of a mechanical pump system, and required the use of a laptop, a thermal camera and an HD digital camera to capture the system while it operated.

The system was designed to simulate the pulsatile blood flow generated by the heart and consists of four major components: A water pump, a solenoid valve, a control box, and three sensors. The system can be seen in Figure 1. The pump is a small submersible pump placed in a water reservoir. It produces enough pressure to push the water through the system at a flow rate of 75 gallons per hour. The pulsatile pumping is simulated by controlling an electric solenoid valve. The solenoid valve defaults to being closed and opens when 12 Volts are

presented across it, in a binary fashion. By carefully controlling the operation of the valve (pattern and timing of opening and closing), a heart-like pulsatile flow was created.

The control box dictates the valve's operation. It houses an Arduino UNO that is programmed to open and close the valve in the pattern specified. The Arduino also handles the outputs of the three sensors in the system: an inline temperature sensor, an outside temperature sensor, and a flow meter that measures the water's flow rate. The data coming out of these sensors is sent serially via a USB cable to be visualized on a computer.

This equipment was designed and built by Tarek Nasser El Harake, who was an undergraduate student at the time of creation. 


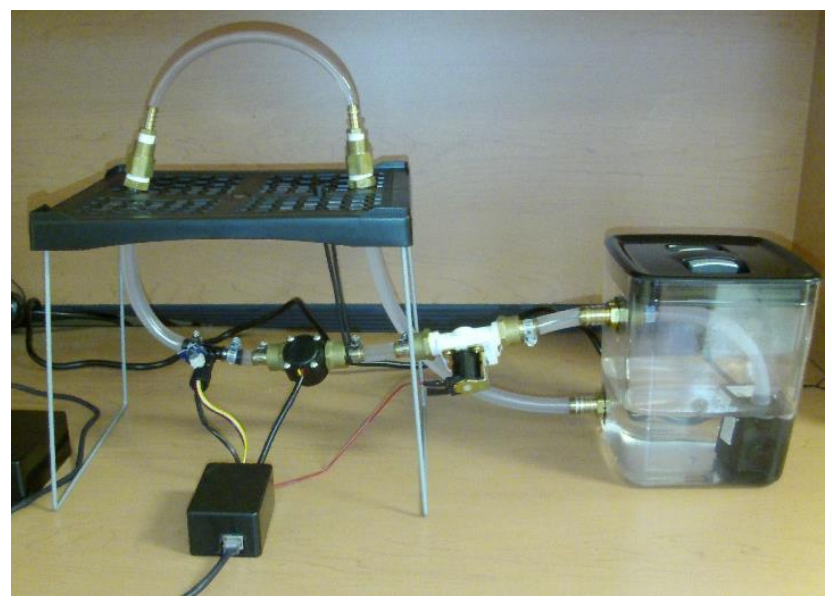

Figure 3.1 Blood simulation model

The A-Series thermal camera and HD digital camera were used to gather video of the flow simulation model while it was operating. The thermal camera is operated via a laptop and associated software.

\subsection{Experimental Conditions}

Most experimentation in this thesis involved the use of thermal imaging. Thermal experimentation involving human subjects are subject to atmospheric interference such as hot or cold air flow, as well as internal interference such as exercise or friction induced heat. Efforts were made to mitigate both internal and atmospheric effects as much as possible by studying guidelines published by the American Academy of Thermology (AAT) as well as the International Academy of Clinical Thermology (IACT) [115],[116].

Several rooms were utilized for experimentation. Older adult patients were examined in their individual hospital rooms. The atmospheric conditions in these rooms were outside of the researcher's control, but on average were measured at $22^{\circ} \mathrm{C}$ and $35 \%$ humidity. Each room was large enough to maintain a homogenous temperature (greater than $8 \mathrm{ft} \times 10 \mathrm{ft}$ ) and did not contain 
florescent lights, which can emit light at wavelengths that border on infrared. During all experimentation, the door to the room was closed to prevent hallway drafts. Before commencement of and between any experimental procedures, the subject was given an equilibrium period of 15 minutes. Timing of data collection was consistent and early in the day, to minimize patient activity and ensure the patient had not been to dialysis prior to data collection.

Healthy adult experimentation all occurred in the same room at Carleton University. This room was set to a temperature of $23^{\circ} \mathrm{C}$ and was measured to have a humidity of $32 \%$ (on average). The room was larger than $8 \mathrm{ft} \times 10 \mathrm{ft}$; large enough to maintain a homogenous temperature. The room did not utilize florescent lights, did not have any windows and had only one door, which was closed for the duration of experimentation. Before any data collection, each subject was given an equilibrium period of 15 minutes and refrained from exercise, sun bathing, physical therapy and the use of creams the morning of experimentation. This minimized the risk of confounding factors, for example, subject sweating, which could affect the emissivity of the skin's surface and affect the accuracy of temperature measurements [117].

\subsection{Experimental Procedures}

Two types of experiments were conducted - those with human subjects and those with none.

These are referred to as simulations. All experimentation is detailed in this section.

\subsubsection{Mobility-Specific Human Trials}

A standard hospital ethics procedure was completed, and approval was obtained to collect data from healthy adult volunteers. Data was collected from these volunteers in two separate ways to capture both discrete and continuous data. The first method involved numerous participants performing several discrete movements. The second method involved two participants 
completing several movements comprising each of two continuous data sets. In both instances, before a participant performed movements, data was collected of pressure exerted on the pressure mats by the mattress alone. This data was used to calibrate and eliminate the weight of the mattress from subject movement data.

Thirty healthy adult participants were recruited to perform discrete movements. This sample size was chosen as it is the minimum required sample size to yield statistically significant results. The participants ages ranged from $20-60$; none were elderly, and none were in-hospital patients. Three movements capture all positional states and state transitions. These three movements are depicted in Figure 3.2 below. Figure $3.2-1$ illustrates a subject beginning in a supine lying state, diverting from this initial position by rolling fully to one side of the body and back to a supine lying position. Figure $3.2-2$ illustrates a subject beginning in a lying state and transferring to a sitting position (this was performed in an exaggerated and slow manner). Figure $3.2-3$ illustrates a subject beginning in a sitting state and transitioning to a standing state. This was completed twice by participants (for the purposes of COP analysis); once in an unstable, slow manner, simulating an elderly patient, and once as a healthy adult normally would. These movements were completed separately, resulting in a total of 120 discrete data files for all thirty subjects. Video footage was captured of each movement for a total of 120 corresponding video files.

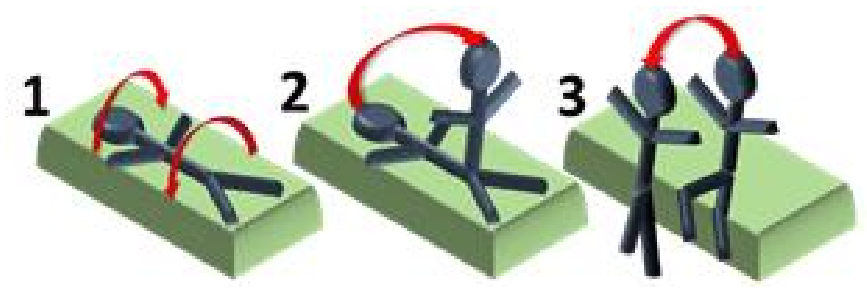

Figure 3.2 Continuous subject movement series. 
Two young, healthy, female non-patient volunteers were recruited to perform a series of movements that constituted four continuous data sets. Each subject began in a supine lying position, then rolled to the left side of the body and back to a supine position as seen in Figure 3.3 - A. Subjects then transitioned from a supine lying position to a sitting position as seen in Figure 3.3 - B. Each of the subject's legs and hips were then swiveled right and moved towards the left (from a bird's eye perspective) side edge of the bed as seen in Figure 3.3 - C. The final movement in the series was a transition from a sitting position at the left edge of the bed, to a standing position as seen in Figure 3.3 - D. These four movements in series constituted one continuous data set. Four continuous data sets were collected, two from each subject.

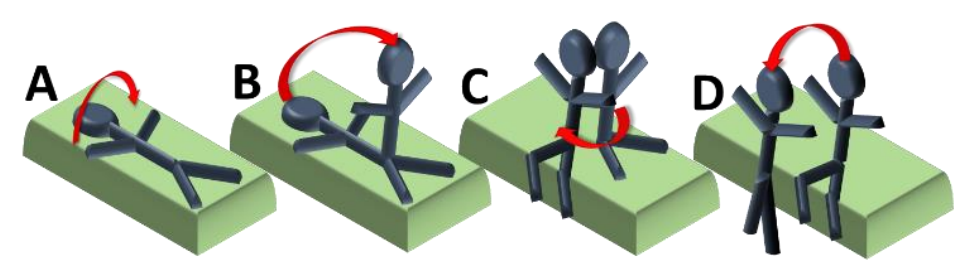

Figure 3.3 Discrete subject movement series.

All mobility specific human experimentation pertains to mobility measurement extraction in Chapter 9.

\subsubsection{Older Adult In-Hospital Data}

Older adult patient data was originally captured with intent to examine pressure ulcer formation and prevention, however throughout the course of data collection, no pressure ulcers fully developed. This data is instead considered in terms of vital signal extraction, focusing on the extraction of pulse and temperature measures. 
The study captured long term pressure data via pressure mats, and both thermal and visible light image data of patients' feet (area of highest hospital incidence) via thermal cameras. Eight older adult patients were recruited and consented to participate in this study, all of which were examined in-hospital. The sample size was based on subject inclusion requirements and a study time frame; the maximum number of subjects was recruited from within the available time frame.

Each patient's bed frame was equipped with two to three pressure mats (depending on the bed frame architecture), which gathered data continuously; one pressure mat under the patient's feet and another pressure mat under the patient's hips. The pressure mats were oriented as in Figure 3.4. The length of the mats is approximately the width of the hospital bed, and so they were placed lengthwise across the width of the bed. Two mats were placed underneath the lower leg area (one underneath the heels and the other underneath the calves), and another mat was placed at the lower back (sacrum). These mats were secured to the bed frame and attached to the data collection box, which was secured underneath the hospital bed. The bed frames were split into sections so the patient position could be automatically adjusted. The mats were placed on sections that remain flat, even when the patient changes position. This ensured the mats would not bend, which would skew data and damage the equipment. The hospital mattress was placed on top of the mats, making the experimental set-up completely nonintrusive. 

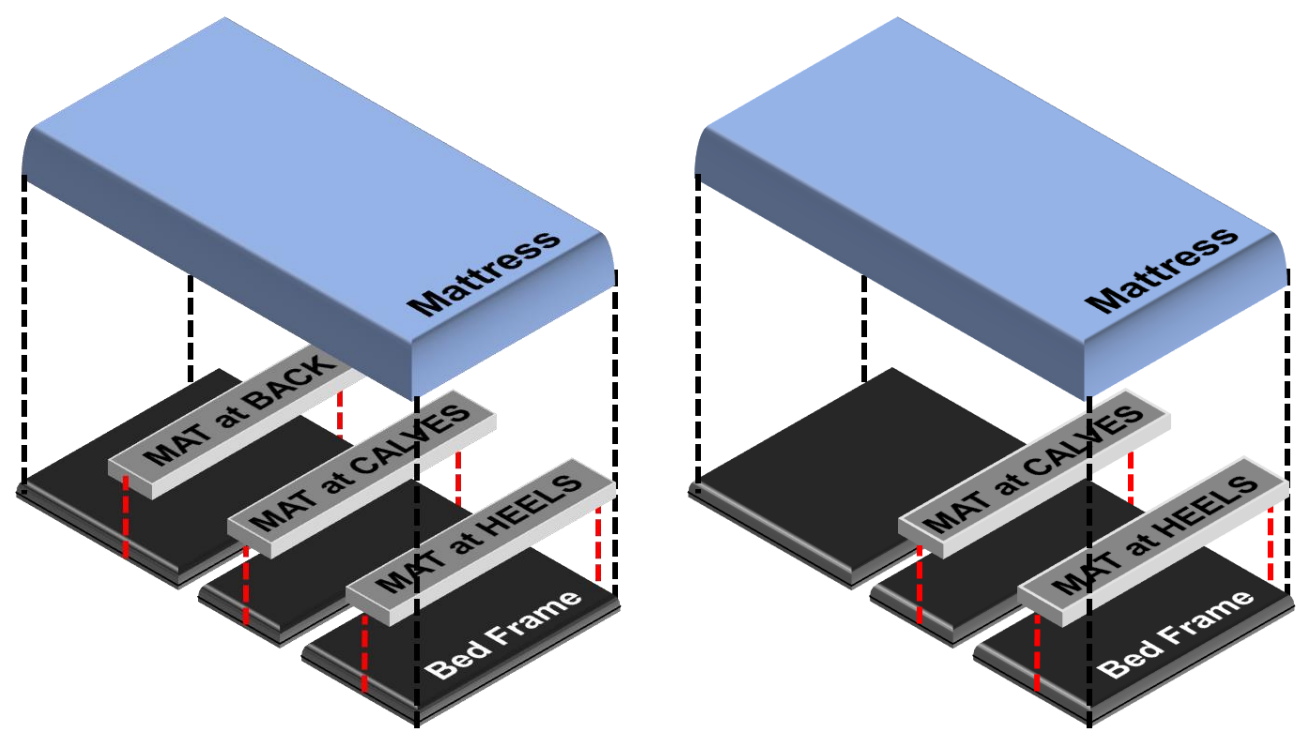

Figure 3.4 Pressure mat placement on patient beds for two and three pressure mats.

Once or twice per week, a researcher visited the hospital to manually capture thermal images of the left and right heels and lateral malleoli. This was completed using the handheld FLIR E4 thermal camera. Individual patients were either sitting or lying (supine, prone or laterally) on any given day. Patient orientation was dependant on a patient's mobility and daily activities; orientation differed amongst patients but were generally consistent for each patient at the time of data collection. The pressure mats were placed so that, regardless of the bed and patient position, the mats were not structurally compromised. The handheld thermal camera allowed the researcher to obtain images no matter the patient orientation. Thermal image data capture was only completed in the mornings, at the same time every day to best control for external factors. While efforts were made to adhere to clinical thermography guidelines, this thesis involves in-hospital patient data gathered for a non-intrusive, non-interventional study. External factors were therefore very difficult to account for. As opposed to accounting entirely for external factors by controlling patient environments and activities, we have accounted for these influences through strategic data collection and analysis. Data collection occurred at 
approximately 10:30am every day; this allowed for an equilibrium period after getting out of bed, minimized the room's exposure to sun and ensured no creams were put on or exercise occurred. Data analysis assumes that for one patient, on any given day, both the left and right feet are subject to the same external influences and examines measures of comparison to best exclude external factors.

Each visit, the researcher captured thermal images of the right heel, right lateral malleolus, left heel and left lateral malleolus. In addition, an image of the room was collected for ambient temperature measurement.

Experimentation with older adults pertains to pulse measurement extraction in Chapter 7 and temperature measurement extraction in Chapter 9.

\subsubsection{Other Data}

Data were collected for this thesis both in hospital as detailed in the previous section, and with healthy adult volunteers performing specified actions in a controlled environment. Fourteen different subjects were examined for vital signal extraction. This sample size was based on time limits; the maximum number of subjects were recruited from within limited time frames. The experimentation with healthy volunteers took place in the same lab, with the same equipment set-up and volunteer procedures specific to the vital sign being studied.

In each case, the subject was first given an equilibrium period of 15 minutes in the experimentation room. The subject was seated in the middle of the room. The thermal and digital cameras were placed on a separate table oriented towards the area of interest on the subject's body, for example, a subject's face. This set-up is depicted in Figure 3.5. The heart rate monitor was initiated, as well as the thermal and digital cameras. For data capture, the 
equipment was initiated manually resulting in staggered start times. To sync the data, noise was introduced to all data modalities simultaneously. The subject was instructed to vigorously shake the heart rate monitor at the same time as nodding the head. Following this movement, the subject sat still and/or performed a series of specified movements depending on the vital sign under examination.
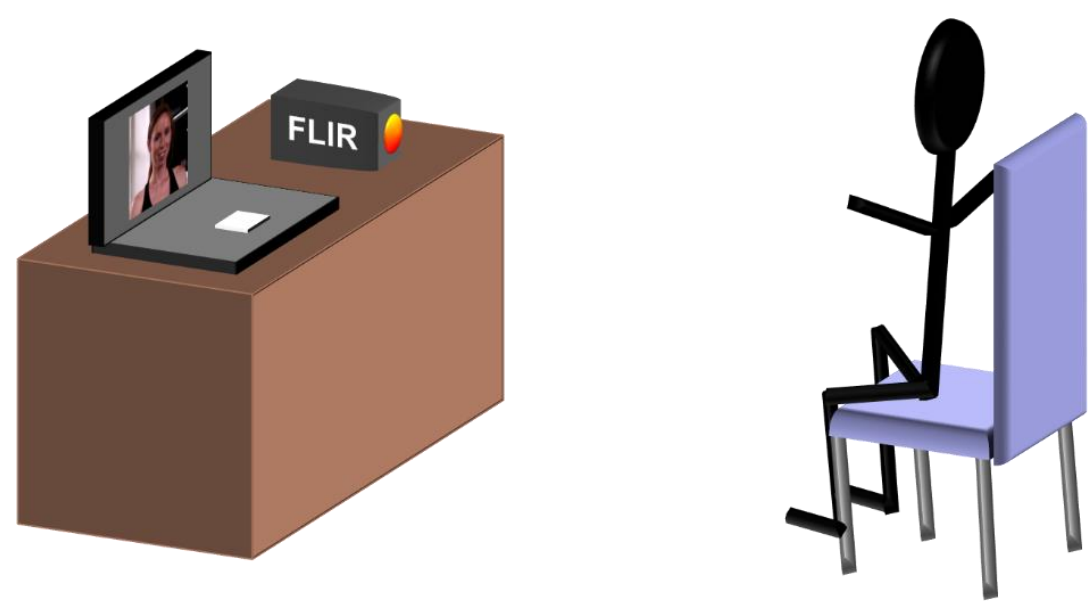

Figure 3.5 Camera and subject orientation.

Pulse measurement experiments pertain to pulse measurement extraction in Chapter 7, and include facial pulse experiments, blood occlusion experiments, pedal pulse experiments and simulation experiments. All pulse measurement experimentation is detailed in this section, except for the simulation experiments which are described in Section 3.6. Fourteen volunteers were recruited for facial pulse measurement experimentation. Upon arrival each subject was seated in the middle of the room and given a 15 minute equilibrium period. The thermal and digital cameras were placed on a separate table oriented towards the subject's face. Following the equilibrium period, the Zephyr BioHarness chest strap was placed around the subject's chest. Following equipment set-up, data collection commenced. The Zephyr BioHarness was initiated, as well as the thermal and digital cameras. The equipment was initiated manually and 
data synchronization occurred as described. Data synchronization was followed with approximately one minute of sitting still. Data collection was then terminated, and all equipment stayed in place.

The blood occlusion experiments were conducted on one subject, immediately following facial pulse measurement data collection. For this experiment, the equipment already in place was used as well as the Qardio wireless blood pressure cuff and the pulse oximeter. The Qardio cuff was placed around the subject's left bicep and the pulse oximeter was placed on their left index finger. The subject was then asked to place their left arm on a heart level surface directly to their left. The digital and thermal cameras were oriented to face the subject's arm, including the pulse oximeter in the digital camera frame. Data collection commenced again as in the facial perfusion experiment; the BioHarness, thermal camera digital camera and pulse oximeter were all initiated. The subject vigorously shook the BioHarness at the same time as shaking the arm and then sat still for approximately one and a half minutes. Directly following the movement, during quiet sitting, the blood pressure cuff was initiated. Both cameras captured the arm as the blood pressure cuff inflated to cut off blood flow and deflated to allow blood flow to return to normal.

The pedal pulse experimentation was conducted with one healthy adult subject. Pedal pulse is an arterial pulse which can be felt on top of the foot (dorsalis pedis artery) or behind the ankle bone (posterior tibial artery). The healthy adult subject was seated in one spot for the duration of data collection. A SonoScape Doppler Ultrasound system was used to extract pedal pulse measurements; this system and its orientation with respect to the foot can be seen in Figure 3.6. The subject was directed to remove their shoes and socks and was given the required 15 minute equilibrium before experimentation commenced on the right foot. The thermal 
camera was placed on the floor, facing the lateral side of the foot. The Doppler Ultrasound system was placed out of the camera view and the transducer was manually placed on the foot to capture blood flow from the posterior tibial artery on the medial side of the foot. This set-up is depicted in Figure 3.7. An experienced individual operated the Doppler ultrasound, while a researcher operated the thermal camera. Once a blood flow signal was picked up by the transducer, video capture of the Doppler ultrasound and the thermal camera began. Approximately 14 seconds of Doppler video data and simultaneous thermal video data was captured.
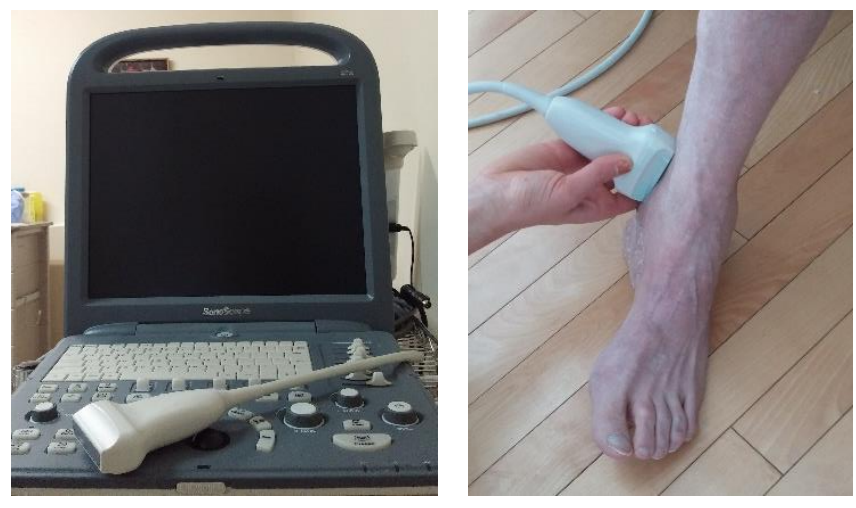

Figure 3.6 SonoScape Doppler System and study subject.

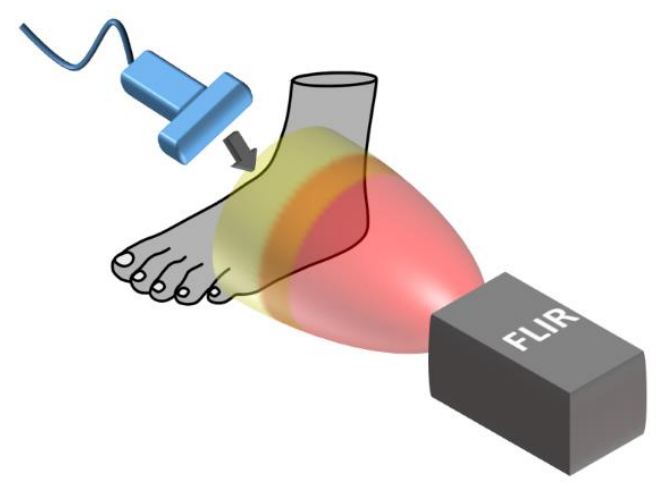

Figure 3.7 The Doppler Ultrasound transducer and thermal camera orientation about the subject. 
Respiration experiments pertain to respiration measurement extraction in Chapter 8 . Respiration experimentation involved data capture of fourteen healthy adult subjects performing a series of breathing actions. For each subject, a 15 minute equilibrium period was given post-experimentation, in which the subject sat quietly. The subject was then asked to continue sitting while the equipment was prepared. The Zephyr BioHarness chest strap was placed around the subject's chest, and the thermal camera was focused on the subject's face. Data synchronization was completed as described. The subject was instructed to vigorously shake the BioHarness at the same time as nodding the head. Following this movement, the subject followed the instructions detailed in Table 3.1.

Table 3.1: Respiration Experimentation Subject Instructions

\begin{tabular}{|c|c|}
\hline Step Number & Instruction \\
\hline 1 & Breathe normally (through the nose) for 60 seconds \\
\hline 2 & $\begin{array}{c}\text { Stop breathing on the next exhale and hold for as } \\
\text { long as possible }\end{array}$ \\
\hline 3 & Return to normal breathing for 30 seconds \\
\hline 4 & $\begin{array}{c}\text { Breathe in a shallow manner, similar to } \\
\text { hyperventilation, for about 15 seconds }\end{array}$ \\
\hline 5 & Return to normal breathing \\
\hline 6 & $\begin{array}{c}\text { Attempt to inhale 2-3 times, heaving the chest, while } \\
\text { closing the throat and then gasp in air }\end{array}$ \\
\hline
\end{tabular}

Body temperature experiments pertain to temperature measurement extraction in Chapter 9 and was explored in healthy subjects in terms of the relationship between temperature and pressure. This is of relevance to vital signal monitoring of an in-bed subject. The relationship 
between temperature and pressure was first examined through application of pressure to subject hands in a controlled manner, to observe temperature changes over time. A pressure mat was therefore used in addition to the thermal and visible light cameras. This pressure mat was placed on the floor. The Bluetooth data transmitter box was attached to the mat via one long wire and kept as far away from the mat as possible to avoid any heat interference. A table was set up in the middle of the room, next to the chair, and an area was indicated in which the subject was to rest their hand after applying pressure.

Following an equilibrium period, the subject sat down and placed their right hand in a supine position in the indicated area. The thermal camera used was the handheld FLIR E4 model, and therefore thermal images for this body temperature experimentation was captured from bird's eye view. This first image was considered the baseline image. The subject then positioned themselves with their knees on the ground and their hands in indicated areas on the mat. The subject then pushed on the mat at a specified pressure level. To ensure this pressure level was approximately achieved, a reference pressure image was generated indicating what respective pressure levels looked like on the real-time display. The subject was able to see this image, compare it to the real-time pressure mat display and ensure that the image they were generating looked very similar to the reference image. An example of this set up can be seen in Figure 3.8. 


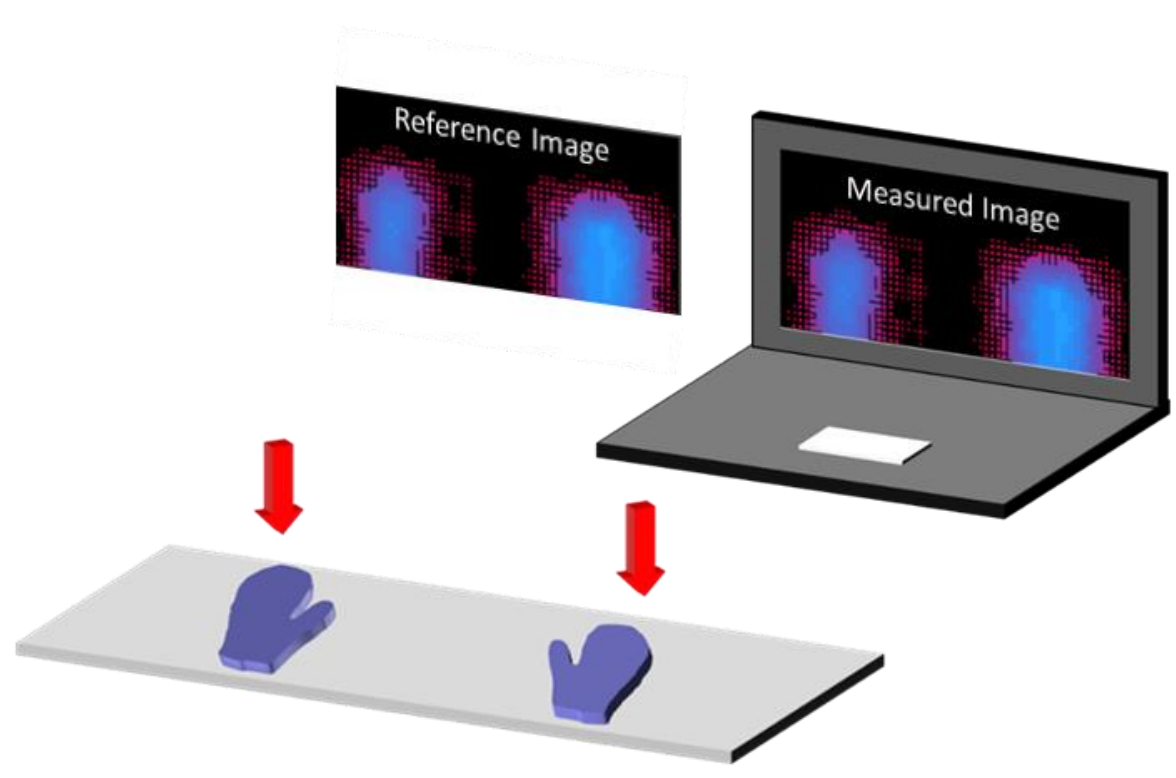

Figure 3.8 Experimental set-up for pressure application.

The subject applied pressure at this specified level for 5 minutes. Directly after this fiveminute period, the subject sat in the chair in the middle of the room, placed their right hand in a supine position in the designated area on the table, and a bird's eye thermal image was taken. The hand remained in this position for ten minutes, and every 30 seconds another thermal image was taken for a total of 1 baseline and 21 pressure recovery thermal images. This entire process was repeated for two distinct levels of pressure; a lighter amount of pressure (average sensor score of 490) and a heavier amount of pressure (an average sensor score of 2020). This procedure is depicted in Figure 3.9. 


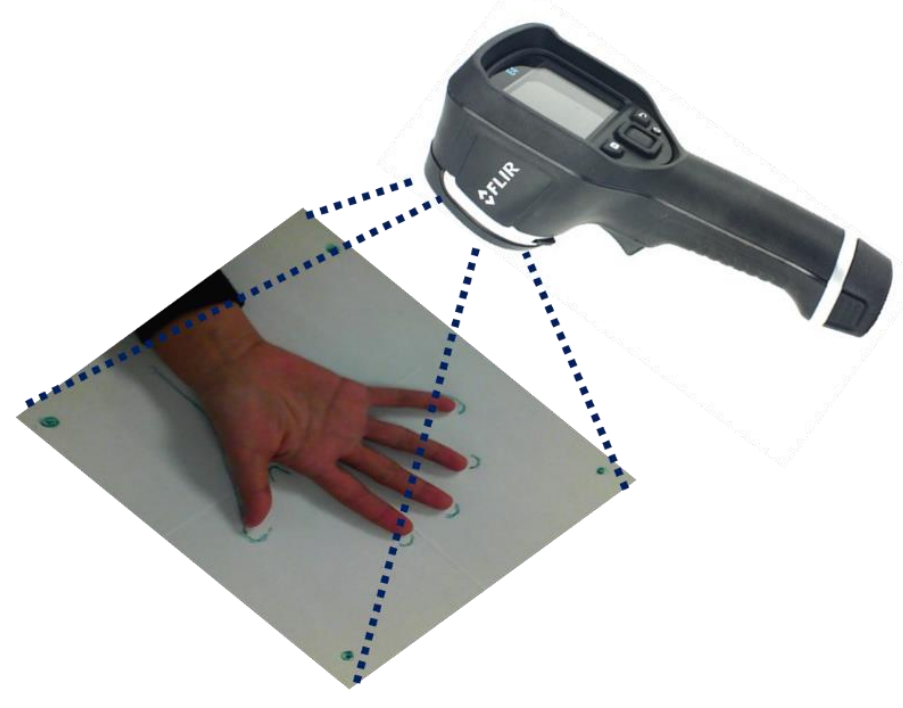

Figure 3.9 Experimental set-up for pressure application.

All data for all experimentation was stored on a secure computer in a locked lab. The following chapters discuss thesis analysis, results and conclusions. Mobility experimentation with healthy adults is presented in Section 3.5.1.

\subsection{Simulation}

For simulation experimentation, the system was set up as depicted in Figure 3.1. The thermal and digital cameras were oriented to face the blood flow simulation system. The system was turned on with room temperature water in the water reservoir. Video data was collected for an approximate minute before hot water was added to the water reservoir. Video data collection continued for another minute as the hot water circulated within the system. Following data collection, water was removed to return the reservoir water level to its initial state. The reservoir was then left until the following day to allow for the system to return to room temperature. This procedure was repeated four more times, for a total of five blood flow simulation trials. 


\section{Chapter 4}

\section{Data Preprocessing and Context Awareness in Vital}

\section{Sign Estimation}

This chapter details the signal and image preprocessing techniques used in this thesis, prior to image segmentation, detailed in Chapter 5. This chapter also discusses context-awareness and details the role of context-awareness within this thesis.

\subsection{Data Pre-processing}

The following sections detail the three main types of data collected, and the data pre-processing techniques applied in this thesis based on previous work.

\subsubsection{Introduction}

Data pre-processing is the removal of noise and compensation for inherent flaws [118]. For example, signals may suffer from random noise and baseline wander and an image may suffer from ambient interference and image artifacts [118]. Removal, or compensation for these characteristics better prepare data for analysis and is particularly important when extracting vital signals as they could be interpreted as the vital signals themselves.

This thesis examines data originating from three different sensor systems; a visible light camera, thermal infrared cameras and pressure mats. The technical specifications for each of 
the described equipment are detailed in Chapter 3. Raw data from equipment have two initial challenges; all sensors are subject to varying amounts of noise, depending on the physics of data capture and the mechanical properties of the system, and the resulting raw data may not be suitable for immediate analysis based on its properties (luminosity, contrast, etc.). This section details the data preprocessing techniques to reduce image noise and enhance images for better vital signal extraction. This stage of the thesis within the scope of the thesis is depicted in Figure 4.1.

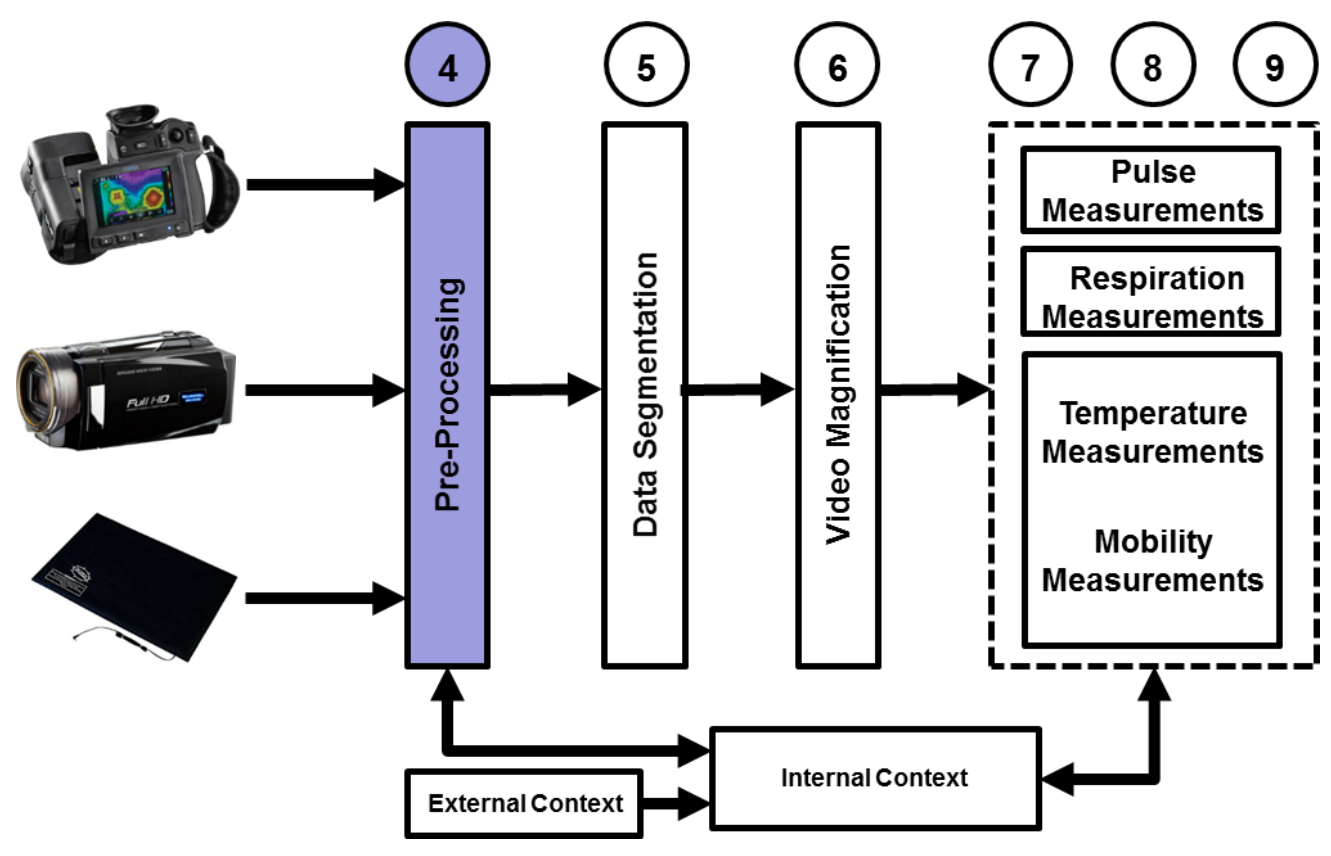

Figure 4.1 Pre-processing stage of the thesis structure

\subsubsection{Thermal Infrared Preprocessing}

Thermal images have some inherent noise due to the nature of the equipment [119]. A median filter with a $3 \times 3$ neighborhood was applied to reduce noise and smooth the image while retaining edge and contour information. Thermal images are also known to have inherently poor contrast [119]. Therefore, following image correction, contrast-limited adaptive 
histogram equalization (CLAHE), with a desired uniform distribution was performed to enhance image contrast, while eliminating artificially induced boundaries and avoiding the amplification of noise.

The raw thermal images sometimes contained watermarks and were captured at varying thermal scales; depending on the thermal camera used (all equipment is detailed in Chapter 3), some variables were not adjustable through the device, only through the device software. For the images that were subject to automatic temperature scaling, the thermal scales were altered to be uniform for all images $\left(20.0^{\circ} \mathrm{C}\right.$ to $\left.35.0^{\circ} \mathrm{C}\right)$ using the FLIR software. The FLIR logo and temperature scale image stamps were removed from affected images by creating a binary image mask, then replacing the nonzero areas with values calculated using an interpolation method based on Laplace's equation. This resulted in the removal of unwanted image stamps and smoothing of the removed areas.

\subsubsection{Visible Light Preprocessing}

Visible light cameras usually suffer from random (not present in the object imaged) variation of brightness or color information in images, which is usually an aspect of electronic noise [120]. It can be produced by the sensor and circuitry of a scanner or digital camera [120]. To reduce this random noise, each frame of a visible light video is smoothed with a low pass Gaussian filter.

\subsubsection{Pressure Sensor Signal Preprocessing}

Pressure data were collected from underneath a hospital mattress, on which lay the subjects of interest: healthy adult volunteers or older adult patients. Pressure data collected from 
volunteers were therefore subject to the filtering effect of the mattress in addition to the weight of the mattress and the subject. Studies completed by Megan Holtzman et. al. indicate that the filtering effect of mattresses does not diminish the ability to extract vital signals and was therefore not compensated for [121]. The weight of the mattress was eliminated as best as possible by collecting one minutes worth of data of the mattress alone, averaging this data for each sensor, and then subtracting these averages from all subsequent data. To account for the random electronic noise, resulting signals were low pass filtered to remove noise while retaining subject movement.

\subsubsection{Conclusion}

This section detailed the data preprocessing techniques used to reduce image noise and enhance images for better vital signal extraction, as described in the following chapters. Thermal images are subjected to median filtering and CLAHE enhancement, visible-light images are subjected to Gaussian filtering, and pressure mat signals are countered for the weight of the mattress and then low pass filtered. These methods were successful in improving the images or signals, and consequently resulted in better vital signal measurement extraction.

\subsection{Context Awareness}

The following sections discuss context-awareness: the type of contextual information considered within the thesis framework, the importance of context awareness within that framework as well as demonstrating context-aware implementation. 


\subsubsection{Introduction}

Context can be considered information that characterizes the state of an entity; an entity being a person, place or thing [122]. Context-aware systems are systems that take in contextual information and adjust accordingly [122],[123],[11]. There are three main types of contextual information: given (user-input information), sensed (data collected intrinsically), and derived (using the information available to calculate, combine or infer additional information) [123]. This thesis examines the role of context-awareness within the thesis framework, as seen in Figure 4.2.

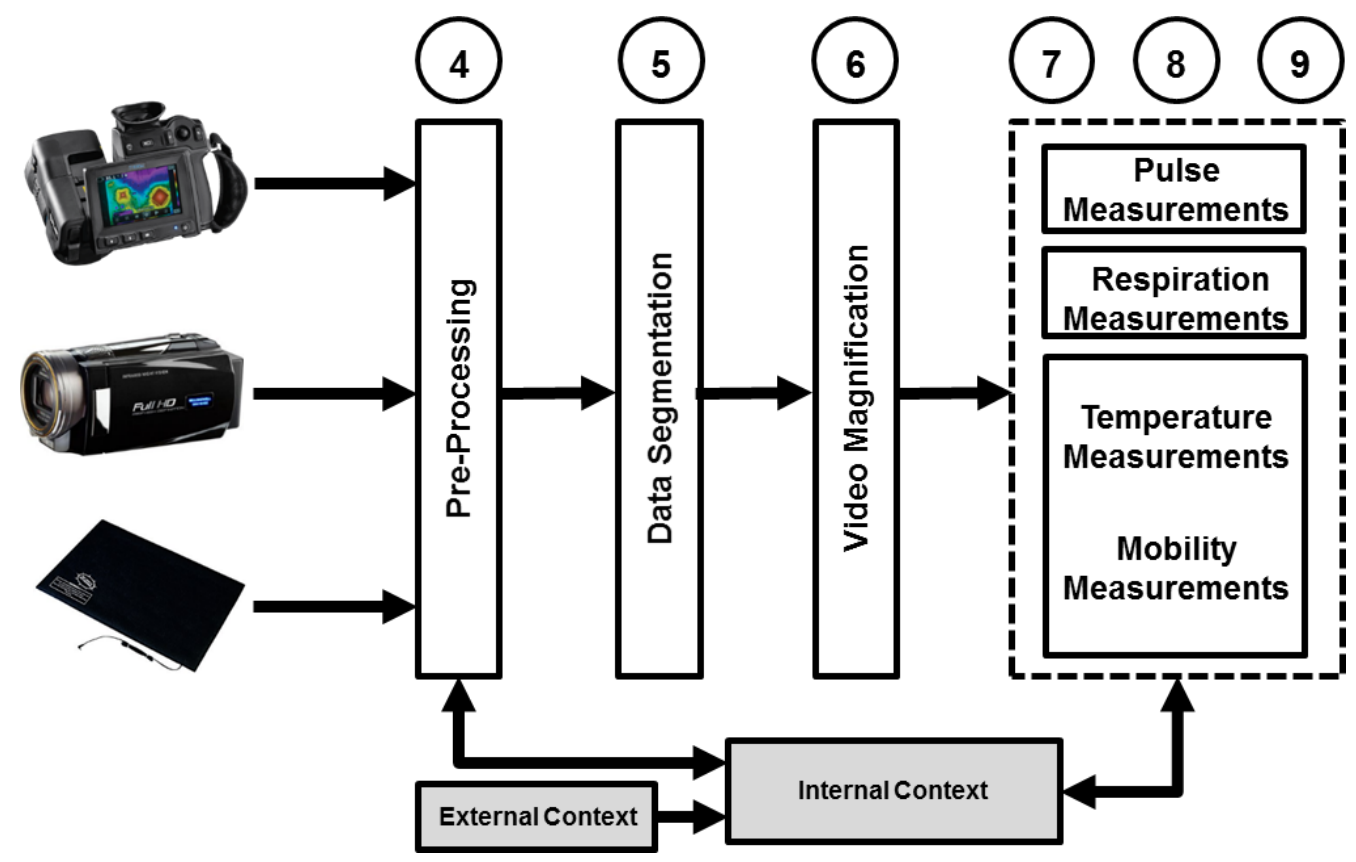

Figure 4.2 Context-awareness stage of the thesis structure

The system framework uses non-contact multi-modality data of a given participant and subjects the input data to several stages of data processing to extract the subject's vital signals. This thesis examines the role of context-awareness in the system, seen working concurrently to the data processing stages. This thesis examines two types of contextual information: 
external context (information introduced to the system), for example contextual data input manually by a user or collected from system sensors (given and sensed, respectively), or internal context (derived by the system), for example calculated from raw data (derived) [122]. This thesis uses context-awareness to facilitate synergy between data types.

There are countless examples of contextual information and system response; all of which cannot be covered within the scope of this thesis. This thesis therefore demonstrates how context-awareness is implemented by examining one particular case in which contextawareness would be required to allow the system to continue functioning. The following sections incorporate context-awareness by examining the behavior of extracted pulse signals from both thermal and visible light cameras when illuminance is diminished to zero lux. In other words, when one modality and respective algorithms fail.

\subsubsection{Contextual Data Processing}

For the examination of context-awareness, thermal video, visible light video and ECG data were collected for an approximate 40 seconds from a 27 year old, healthy, female subject. The specifics for experimental conditions and procedures can be found in Chapter 3 .

Each frame from each video was first subjected to pre-processing as described in Section 4.1. To test the viability of context-awareness within the proposed system, pulse measurements were extracted from thermal and optical video as one modality and respective algorithms were driven to failure. This was achieved by first transforming visible light video from the RGB color space to the YIQ color space, and iteratively decreasing the intensity to zero over an approximate 35 seconds before transforming back to the RGB color space. After reducing the intensity value, the resulting level was quantized to 8 bits. The video was light altered through 
video processing (as opposed to the use of a dimmer, for example) for a controlled and calculated decrease in light intensity.

Following pre-processing and light-adjustment, each frame from each video was subjected to image segmentation to identify the anatomical location from which to extract pulse measurements. Both visible light and thermal data was segmented using binary masks; each mask was used to create regions of interest (ROIs) on the subject's face. The anatomical location of interest was chosen to be the forehead based on previous work by of Hamedani et. al. [68], Buddharaju et. al. [43] and Bennett et. al. [20]. These ROIs are depicted in Figure and were applied to all optical and thermal videos of the subject's face, respectively.

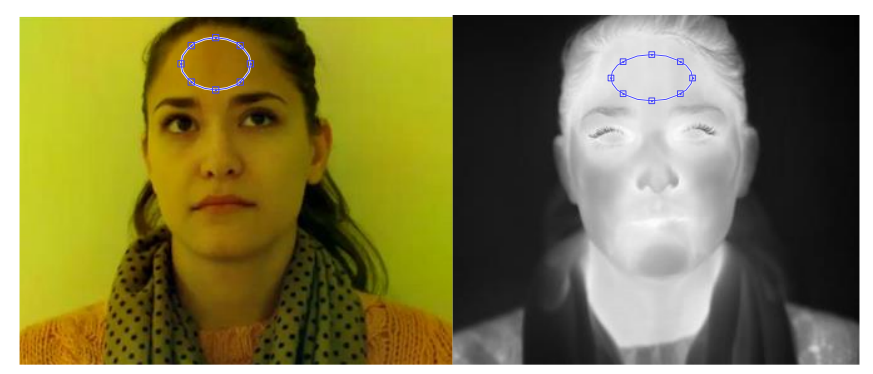

Figure 4.3 Visible light and thermal image segmentation

The next stage of data processing was video magnification. An adaptive algorithm was developed for the system framework, based on the Eulerian Video Magnification (EVM) method, developed at MIT [9]. This algorithm iterates the EVM processing twice; once with a wide temporal band-pass filter to identify an underlying frequency, and again with a narrower filter window to enhance the signal of interest [20]. This results in the extraction of amplified vital signals from subjects, instead of amplified noise.

Following video magnification, the enhanced signals are extracted from the ROIs (depicted in Figure 4.3). This consisted of calculating the mean intensity from within each region of each 
visible light and thermal video frame. These signals, as well as the pre-light adjusted signal, were examined, and performance was quantified by finding numbers and locations of peaks.

\subsubsection{Context-Aware Results}

The video preprocessing removed equipment induced noise, and CLAHE successfully enhanced contrast in the thermal video frames. The thermal and visible light cameras were tested for their response to light by capturing a subject when the (windowless) room lights were on, and when the lights had been turned off. This test produced the expected visible light image results (the participant was not visible when the lights were turned off), and the thermal image results depicted in Figure 4.4. These results support the hypothesis that, in the absence of light, the visible light camera fails while the thermal camera continues to collect data.

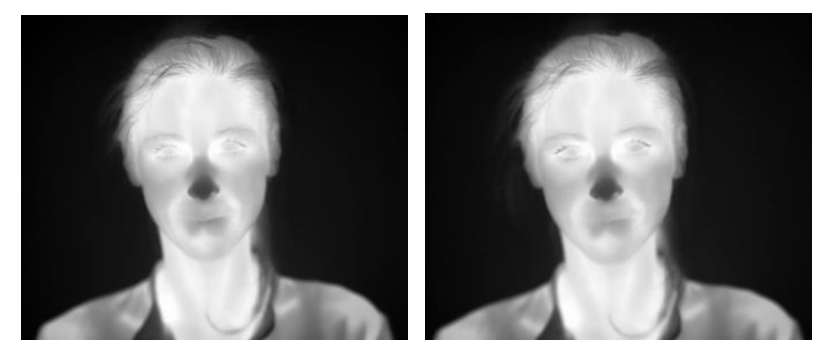

Figure 4.4 Thermal image with lights on (left) and lights off (right).

The pulse measurements extracted from the EVM enhanced thermal video and intensity altered visible-light video are depicted in Figure 4.5. It is clear that, in diminishing light, the algorithms designed to extract pulse measurements from visible-light video data fail. The point at which these algorithms fail is at approximately 24 seconds whereas the intensity for all respective video frame pixels reaches zero at approximately 35 seconds; failure occurs at 15\% of the original light condition. The thermal video is unaffected by changes in light conditions; the associated algorithms continue to extract pulse measurements in the absence of light. 


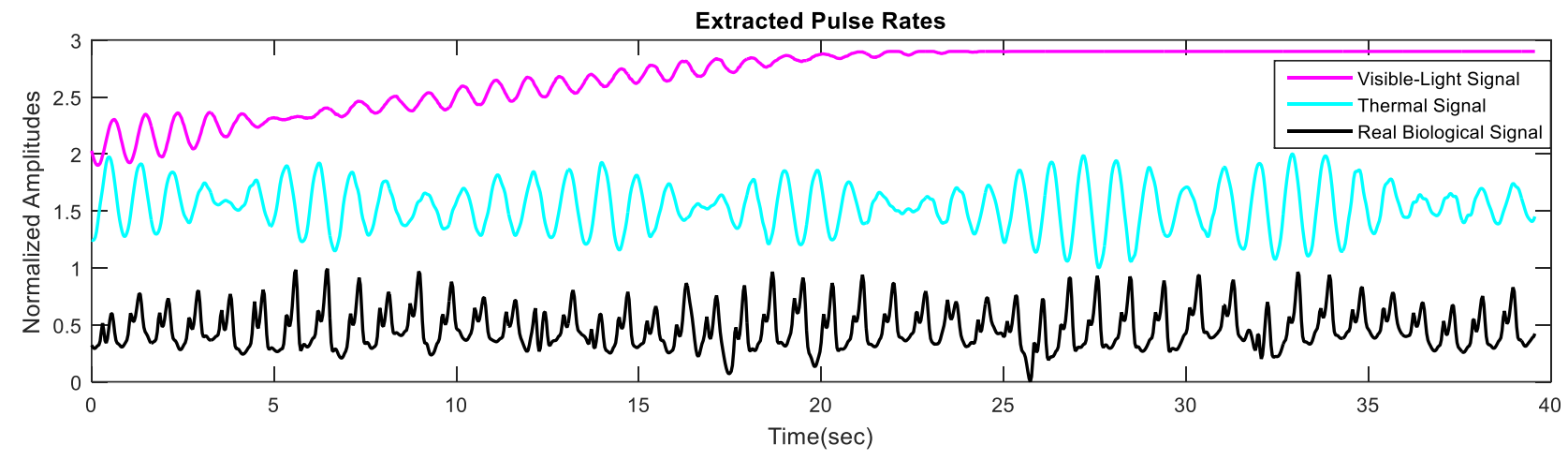

Figure 4.5 Extracted pulse measurements from thermal and visible light data, as illuminance was reduced to zero

This result depicted in Figure 4.5 was expected and demonstrated a scenario in which context-awareness in this system could improve error, reliability and processing time. This system would detect the failing signal and remove the visible-light data as an input to the system.

While this chapter explores one example of context, there are many types of context relevant to this thesis. For example a subject who has been stationary under blankets for a long period of time may present an issue in the thermal camera and respective algorithms in distinguishing between skin and blankets, especially because thermal cameras are inherently weak in image contrast. In such a scenario, the vital signals would report as not present. The visible light camera could be used to distinguish between skin and blanket, and therefore reduce error in the system. 


\subsubsection{Conclusion}

This thesis considers context-awareness as part of the system framework. This section used a specific instance to demonstrate the potential for context-awareness to improve the existing system framework by reducing error and processing time. 


\section{Chapter 5}

\section{Data Segmentation in Vital Sign Estimation}

This chapter details image segmentation techniques used in this thesis, following signal and image processing, detailed in Chapter 4, and prior to spatio-temporal processing and signal extraction, detailed in chapters 6 and 7.

\subsection{Introduction}

In the extraction of vital signs from raw data, data is first de-noised and inherent signal flaws are corrected, as described in Chapter 4. Following this pre-processing, data regions containing the desired information need to be identified. In images, this means identifying the body, and the specific area of the body from which to extract a signal. In pressure sensor signals, this means identifying the sensors of interest based on spacial orientation. This chapter describes the methods used to identify regions of interest in the thermal images, visible-light images and pressure sensor signals for each of four vital signs; pulse, respiration, mobility and temperature. This stage of processing is depicted in Figure 5.1. 


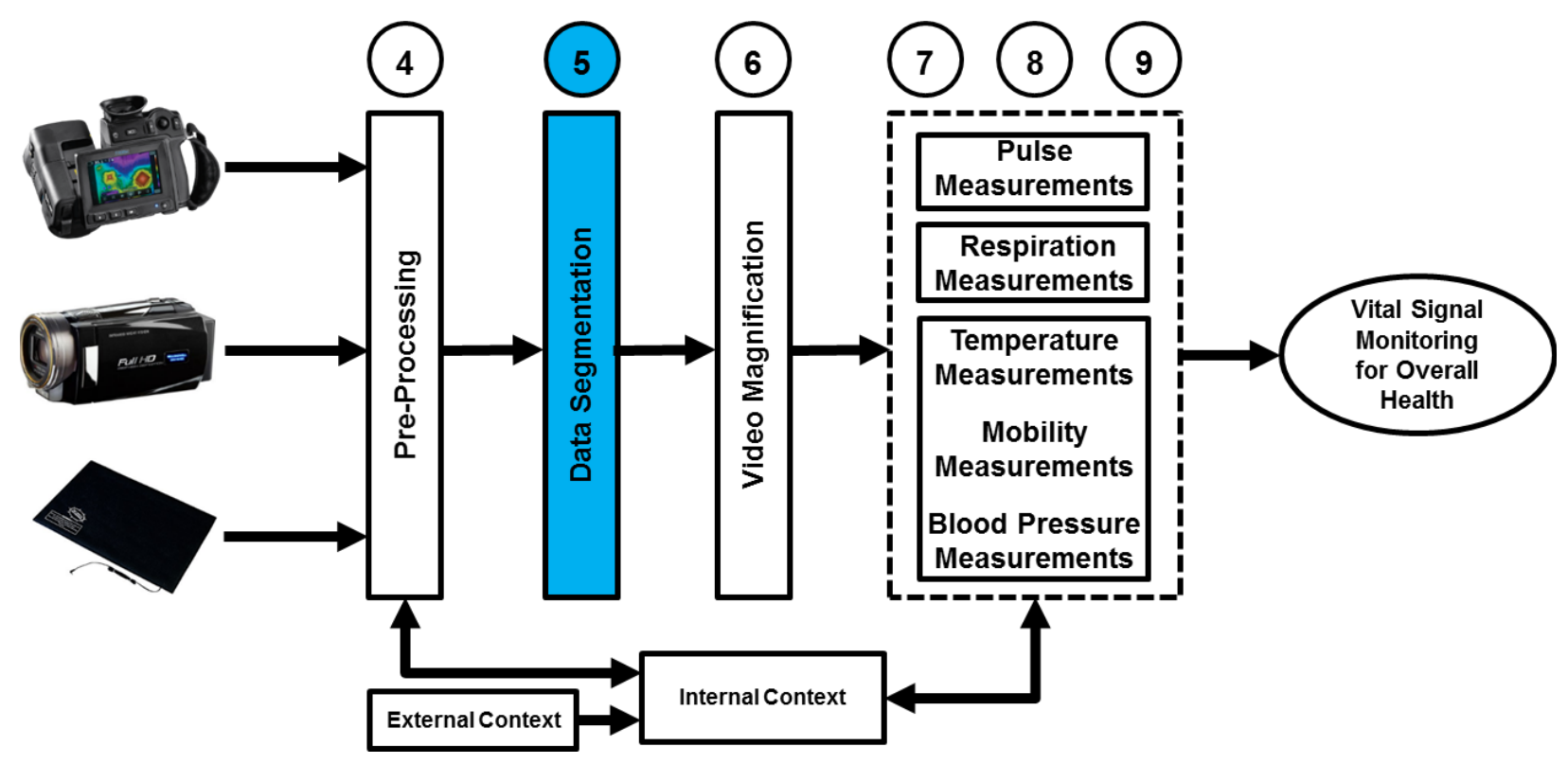

Figure 5.1 Data segmentation stage of the thesis structure

\subsection{Pulse Measurements}

This thesis examines pulse measurements in two ways: theoretically, using a mechanical system simulating blood flow, and real blood flow in human subjects. The details of pulse measurement experimentation can be found detailed in Chapter 3. This section details the thermal infrared and visible light image segmentation methods used to identify the regions of interest in both the mechanical system and human subjects.

The mechanical system was designed to simulate pulsatile blood flow generated by the human heart. The aim of the experimentation was to measure pulsatile flow when hot water was introduced to the system, and therefore the region of interest was identified to be the apex of the tube arching above the black plastic platform. This ROI can be seen in Figure 5.2. 


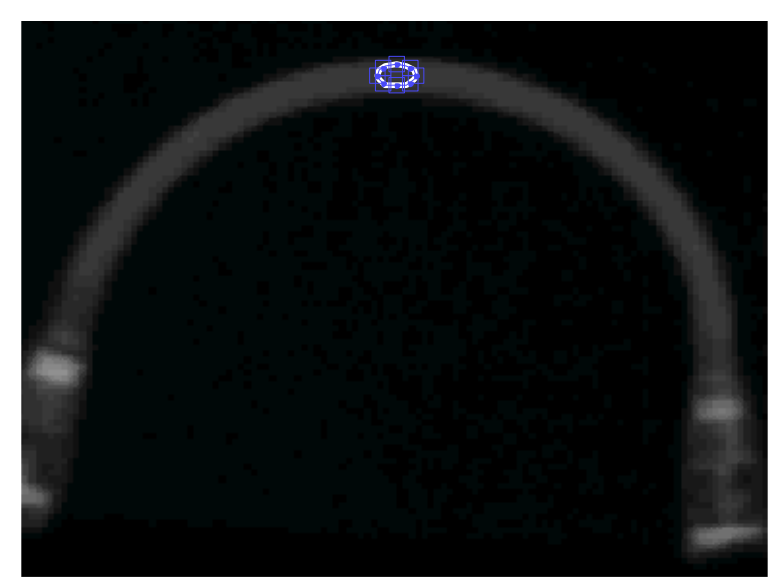

Figure 5.2 Blood simulation model ROI

Human subject pulse measurements can be extracted from several different areas of the body. In this thesis, the extraction of pulse is examined proximally (to the heart) and distally. Regions of interest for pulse measurement extraction were chosen based on previous work and/or anatomical orientation of vasculature. In this thesis, proximal measurements were extracted from the head, neck and chest. Studies have shown that pulse measurements can be extracted from these body parts with great accuracy [68],[43]. Distal measurements were extracted from subjects' feet, a region not currently extensively examined. Pulse measurements were also examined through specific blood vessels while blood supply was occluded and relieved. This measurement occurred at the forearm, while a blood pressure cuff was used to compress the blood vessels in the arm and then relieve them. The following details the image segmentation methods used to identify the regions of interest proximally, distally and during blood occlusion.

The proximal pulse measurement experimentation group consisted of data capture of subjects' head and shoulders, with the goal of determining whether blood flow can be detected proximally with a thermal camera as well as a visible light camera. This chapter examines the best region to extract pulse measurements from. Binary masks were used to create regions of interest 
(ROIs) on each subject's face. Many ROIs were initially explored, some examples of which are displayed in Figure 5.3 and Figure 5.4. Two locations were chosen based on the work of Hamedani et. al. [68], and Buddharaju et. al. [43]; the forehead and the jugular artery. In some parts of this thesis (Chapter 6), the chest was used, however this region is not likely to be used in a hospital setting, as the chest is not consistently visible on in-bed subjects, and often obscured by blankets. The forehead and jugular ROIs are depicted in Figure 5.4 and were applied to all thermal and optical videos of the subject's face. While pulse measurement signals extracted from both ROIs were indicative of blood flow generated by the heart, the forehead ROI resulted in a signal best representative of heart signals.

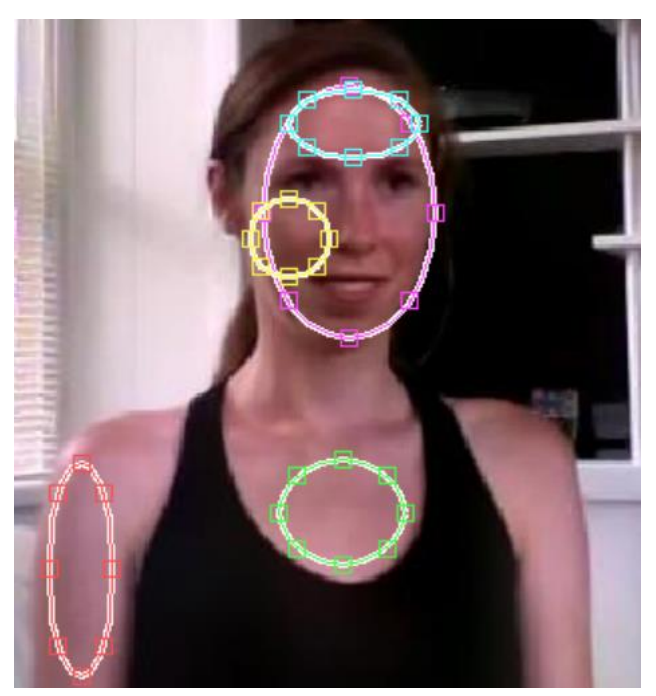

Figure 5.3 Depiction of the light video ROIs 


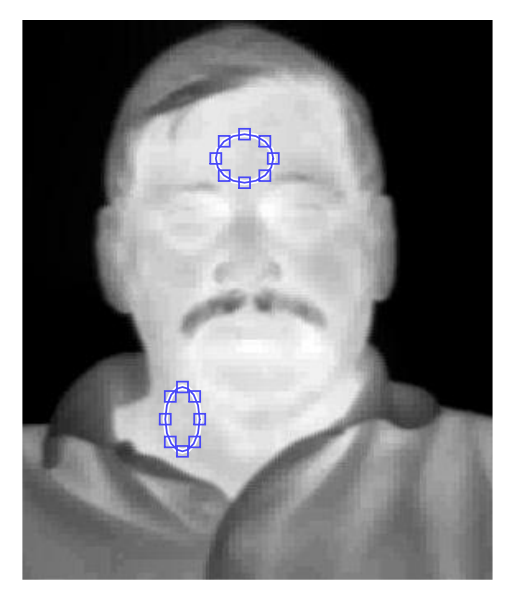

Figure 5.4 Proximal Perfusion ROIs.

The extremities pulse measurement experimentation group consisted of data capture of subject feet, with the goal of determining whether blood flow can be detected at the extremities. In this chapter, the focus is on the best regions to extract pulse measurements from. Two subjects were examined for extremity pulse measurement extraction; one healthy adult subject, and one older adult, diabetic, dialysis patient. The patient had one significantly colder heel, as can be seen in the top two subplots of Figure 5.5, which can indicate blood flow imbalances indicative of inflammation or blow flow constriction. This pattern is in contrast to the healthy adult's heels, which are comparatively symmetrical with respect to temperature distribution. The healthy adult's heels can be seen in the bottom two subplots of Figure 5.5. Signals resulting from different ROIs were therefore examined specifically for patterns indicative of microvascular health. 

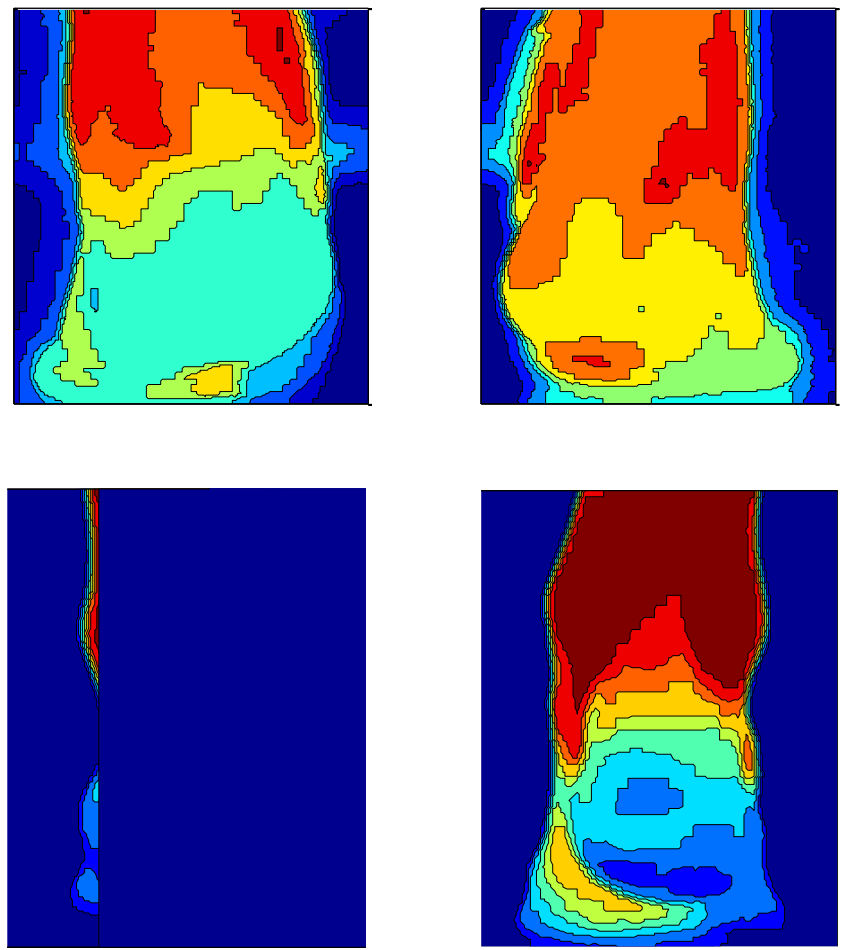

Figure 5.5 The patient's asymmetric temperature distribution of the heels (top plots) versus the healthy participant's symmetric temperature distribution of the heels (bottom plots).

For healthy adult volunteer feet, regions were chosen based on the anatomy of a foot; areas close to exposed veins were favored and bony prominences were avoided. These ROIs include the top of the foot, underneath the lateral malleoli, behind the fibula (posterior) and in front of the fibula (anterior) as seen in Figure 5.6. For patient heels, six ROIs were created using binary masks, each ROI enclosing a different anatomical area. These areas included the heel, the area behind each lateral malleoli and the area behind each medial malleoli. These ROIs can be seen in Figure 5.7. 


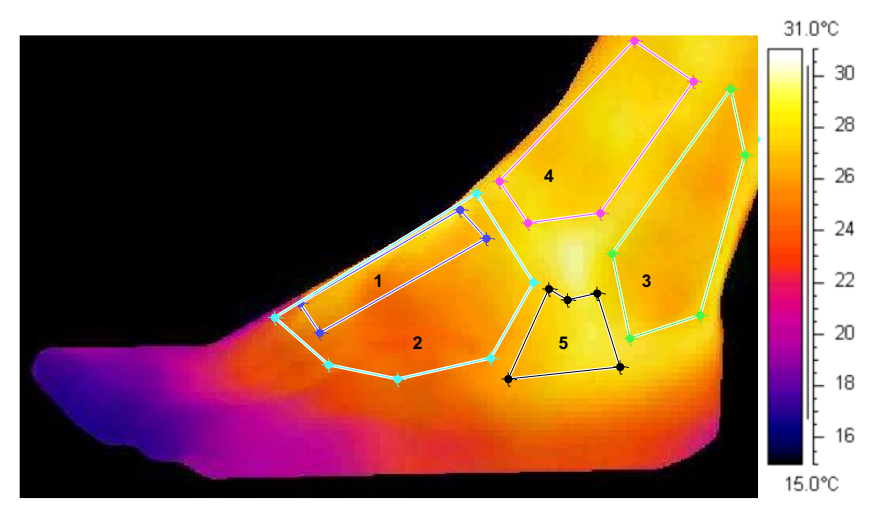

Figure 5.6 Regions of interest on the healthy adult's lateral side of the foot.

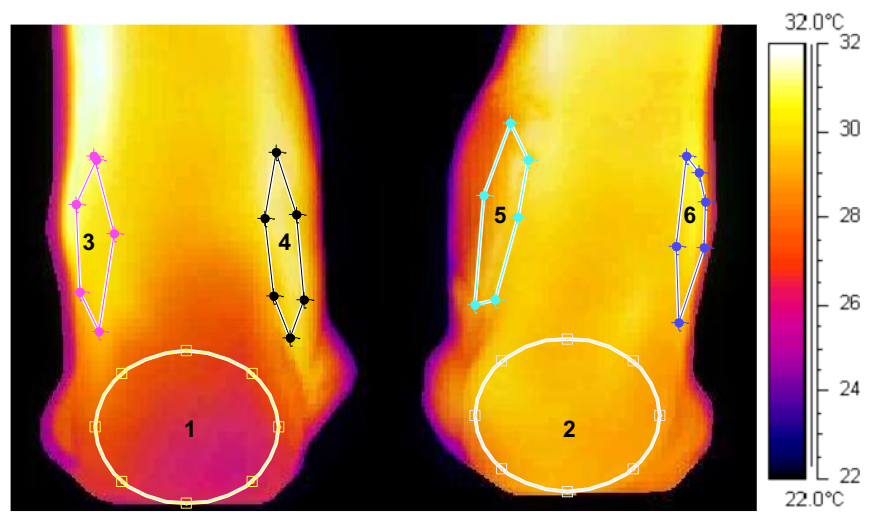

Figure 5.7 Regions of interest on the patient's heels.

Of the five healthy adult ROIs seen in Figure 5.6, two resulted in mean intensity pedal pulse estimates with the same frequency as the measured pedal pulse; regions 1 and 2 . Of these two regions, region 1 produced a signal with a larger amplitude. Regions 1 and 2 both contain the Dorsalis Pedis Artery, which is superficial and runs along the top of the foot. Region 1, the region resulting in the strongest signal amplitude, is directly on top of and area-limited to the Dorsalis Pedis Artery. If the desired signal is detected at the arteries, the Signal Noise Ratio (SNR) is high at these areas. Conversely, the SNR is lower in other areas, resulting in amplified noise. These region-specific results suggest that thermal video, in conjunction with video processing can be 
used to extract a signal representative of pedal pulse, specifically from areas where arteries are close to the skin (areas of high SNR).

Of the six patient ROIs seen in Figure 5.7, three ROIs resulted in mean intensity pedal pulse estimates of $1.1 \mathrm{~Hz}$ (66 beats per minute); these ROIs included regions 2, 4 and 5 . Three ROIs resulted in mean intensity pedal pulse estimates of $1.2 \mathrm{~Hz}$ ( 72 beats per minute); these ROIs included regions 1, 3 and 6 . The largest difference in mean intensity signals of the same frequency was between the signal amplitudes of regions 4 and 5 . These results are supported by the anatomy of the foot. The posterior tibial artery runs down the calf on the medial side, behind the medial malleoli and then towards the toes, underneath the arch of the foot. This artery supplies blood to the heel and is closest to the skin at the medial malleoli. The results indicate that the largest difference in signal amplitudes was between the areas enclosing the posterior tibial artery on the left and right feet (behind the medial malleoli). The signal resulting from the region enclosing the left foot posterior tibial artery was much weaker than from the right. In other words, the weaker signal was on top of the artery supplying blood to the colder heel. The differences in pedal pulse estimates (1.1 Hz versus $1.2 \mathrm{~Hz}$ ) may be the result of differences in SNR at these areas. The areas enclosing arteries may have a higher SNR than the areas that do not. Video enhancement in the former instance is likely to have revealed the pedal pulse while the latter group is likely the result of enhanced noise. While the pedal pulse of the patient was not measured at the time of thermal video capture, the healthy adult results strengthen this theory.

Blood occlusion was examined to determine how sensitive thermal cameras were to realtime blood flow and blood flow abnormalities. This supports the hypothesis that the pulse measurements extracted in this thesis are due to blood perfusion. The experimentation group consisted of data capture of subject's arms as a blood pressure cuff occluded and relieved blood 
flow. The arm ROIs were chosen to be over the brachial artery and the arteries at the wrist (ulnar and radial) as these are areas of superficial veins with strong pulses. These ROIs are depicted in Figure 5.8 and were applied to all thermal and optical videos of subject's arms.

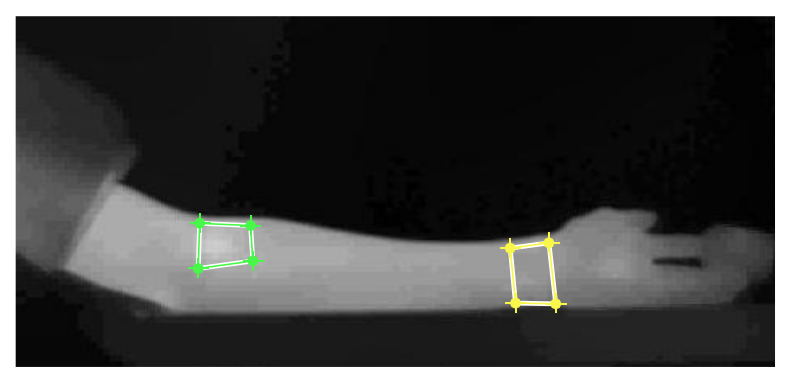

Figure 5.8 Blood occlusion ROIs.

In further analysis of pulse measurement during blood occlusion, image segmentation is used to examine the specific temperature patterns of blood vessels, as opposed to the general pattern of blood flow and perfusion through an area of skin. Thermal video was captured of the forearm area as a blood pressure cuff occluded blood flow and then relieved pressure, allowing flow to return. Each frame of each thermal video depicts an arm, with several visible veins undergoing vascular occlusion. An example of one frame can be seen in Figure 5.9, as well as the identified blood vessels. Each frame was subjected to three image segmentation techniques; basic thresholding, watershed transform and level set method. These segmentation approaches are detailed as follows. 


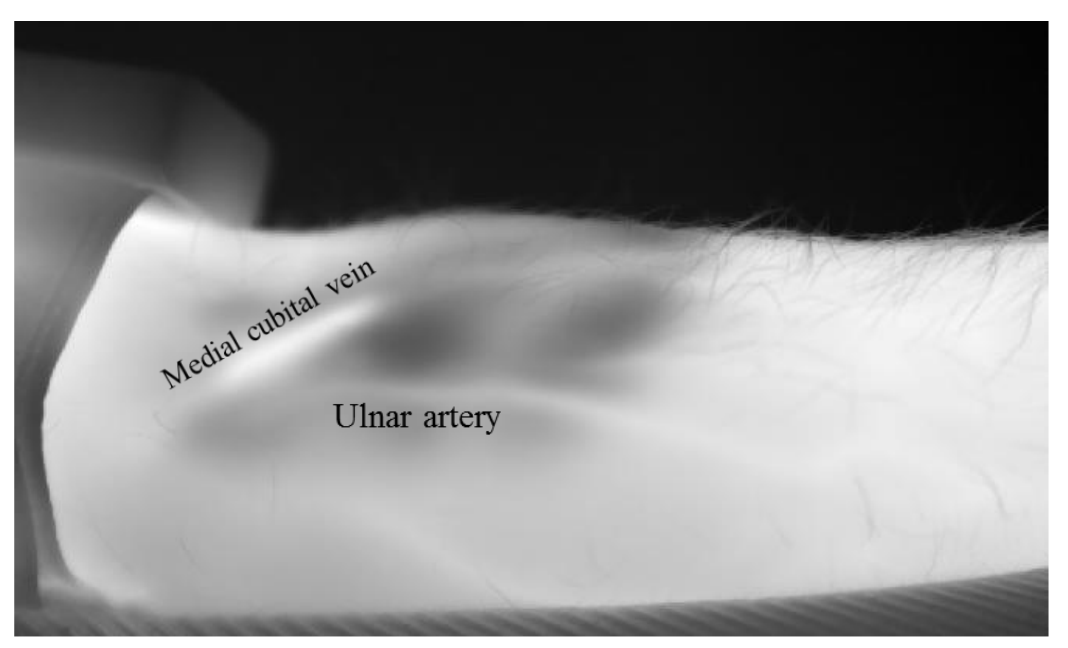

Figure 5.9 Vasculature of interest.

Basic thresholding processing involves first cropping each frame to contain the area of interest and transforming these frames from grayscale to binary using a defined threshold. This threshold was user specified and separated the foreground (blood vessels) from the background (skin and hair). Morphological opening was performed to remove extraneous objects and the resulting binary images were used as an input to the watershed and level set methods.

Watershed processing uses the output frames from the basic thresholding method as an input to the watershed algorithm, which finds basins, and then watersheds by taking the set complement. The algorithm first computes the distance transform of the binary image complement and forces the background to be a catchment basin by setting the background pixels to negative infinity. To avoid over-segmentation, regional minima were imposed before applying the watershed transform.

Level Set processing also uses each output frame from the basic thresholding method as an input to the Distance Regularized Level Set Evolution (DRLSE) algorithm, as developed by and detailed in [124]. The DRLSE algorithm first initiates the zero level as a square surrounding the 
region of interest (this thesis initiates the zero level contour using the output from basic thresholding), and computes the gradient magnitude of the image. The algorithm then iteratively updates the level contour to minimize the gradient flow energy equation. The DRLSE formulates the level set with a distance regularization term and an external energy term. The distance regularization term maintains a desired shape, calculated using the gradient magnitude. The external energy term drives the iterative contour in certain directions (this thesis sets this external force to zero).

The binary, thresholded image of each video frame was the input to the watershed and DRLSE algorithms. The basic, watershed and DRLSE segmentation methods all resulted in successful identification and segmentation of the blood vessels but varied in performance. This is illustrated in Figure 5.10 to Figure 5.12; each figure depicts the segmented regions resulting from each method at three distinct times. The blood pressure cuff was fully deflated at 40s, and blood flow was regular. The blood pressure cuff reached maximum pressure exertion at approximately 80 seconds, and the pulse oximeter lost signal at around 85 seconds (indicating a lack of blood flow). By 110 seconds, the blood pressure cuff had fully deflated again. The segmentation results from the basic, DRLSE and watershed methods can be seen at 40, 90, and 110 seconds in Figures Figure 5.10 to Figure 5.12, respectively (representing regular blood flow, blood occlusion and vascular recovery). 
Basic, Level Set and Watershed Segmentation at 40s

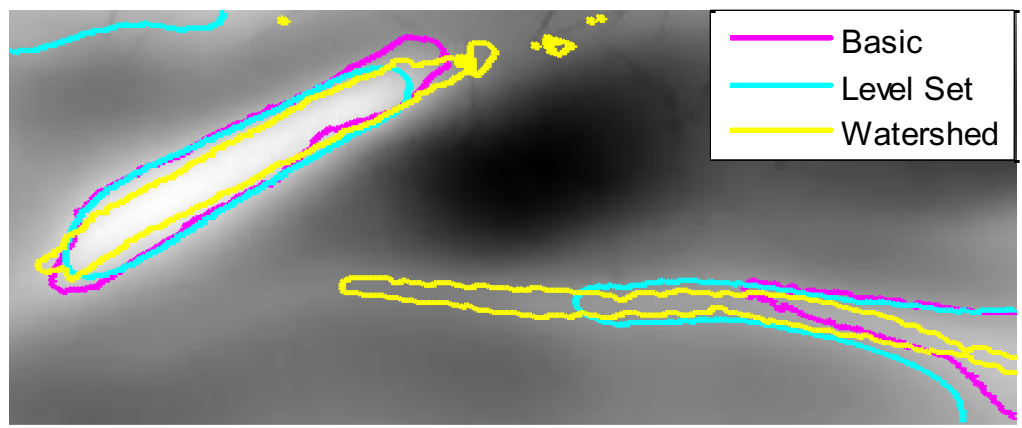

Figure 5.10 The segmentation results at 40 seconds. This was a time at which the cuff had not inflated. The vein is visible and blood flow through the artery is normal.

Basic, Level Set and Watershed Segmentation at 90s

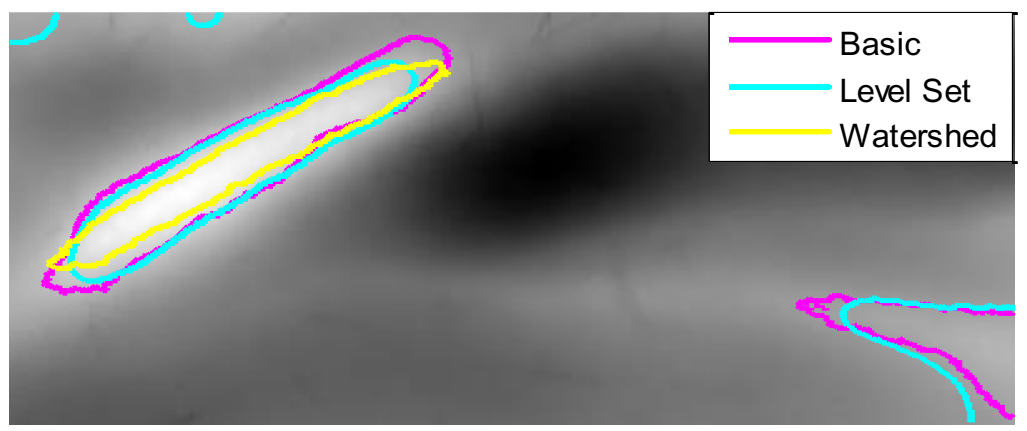

Figure 5.11 The segmentation results at 90 seconds. At this time, the cuff was fully inflated. The vein is still visible, artery is no longer receiving blood.

Basic, Level Set and Watershed Segmentation at 110s

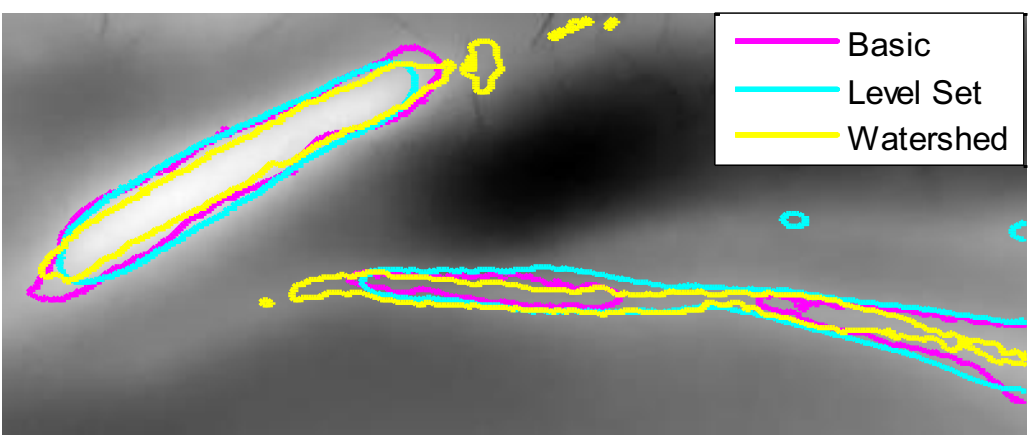

Figure 5.12 The segmentation results at 110 seconds. The blood pressure the cuff had stopped operating and was deflated. The vein remains open and blood is flowing through the artery again. 
When the method results are compared visually, the basic method appears to overestimate the region boundaries containing the median cubital vein (left region) and underestimate the region boundaries containing the ulnar artery (right region). The level set method appears to maintain a more correct shape (smoother boundaries with less holes) than the basic or DRLSE method, and the DRLSE method appears to best estimate the size of the regions. Both the DRLSE and watershed methods identify regions not part of the vasculature.

In examining image segmentation results, a gold standard is generally used as a comparison in which an expert manually identifies the regions of interest. This was difficult to accomplish in this study, as an x-ray would be required to indicate this specific subject's vasculature. This study therefore considered a measure of results to be as compared to vascular anatomy as well as what is known to happen to vasculature in the forearm due to a blood pressure cuff. Figure 5.13 depicts the vasculature of the forearm. This general vasculature was compared to thermal images to identify which areas represented which vascular entities. The behaviour of these areas in response to blood occlusion was also informative in identifying specific vessels. The behavior of specific vessels in response to blood occlusion and relief is known; Figure 5.14 depicts a subject's measured brachial artery diameter (the brachial artery splits into the radial artery and the ulnar artery) as the artery is occluded by an operating blood pressure cuff and then relieved. There is a dramatic change in arterial diameter when the blood pressure cuff deflates. While this is not the same measurement examined in this section, it gives an idea of the expected blood flow patterns. The ulnar artery should first cease to receive any blood due to occlusion, and then, upon reperfusion, should show a notable increase in blood flow, even exceeding what was initially measured. The blood flow patterns are examined here in terms of image segmented thermal 
images, where the best performing methods should mirror known blood flow patterns in response to an operating blood pressure cuff.

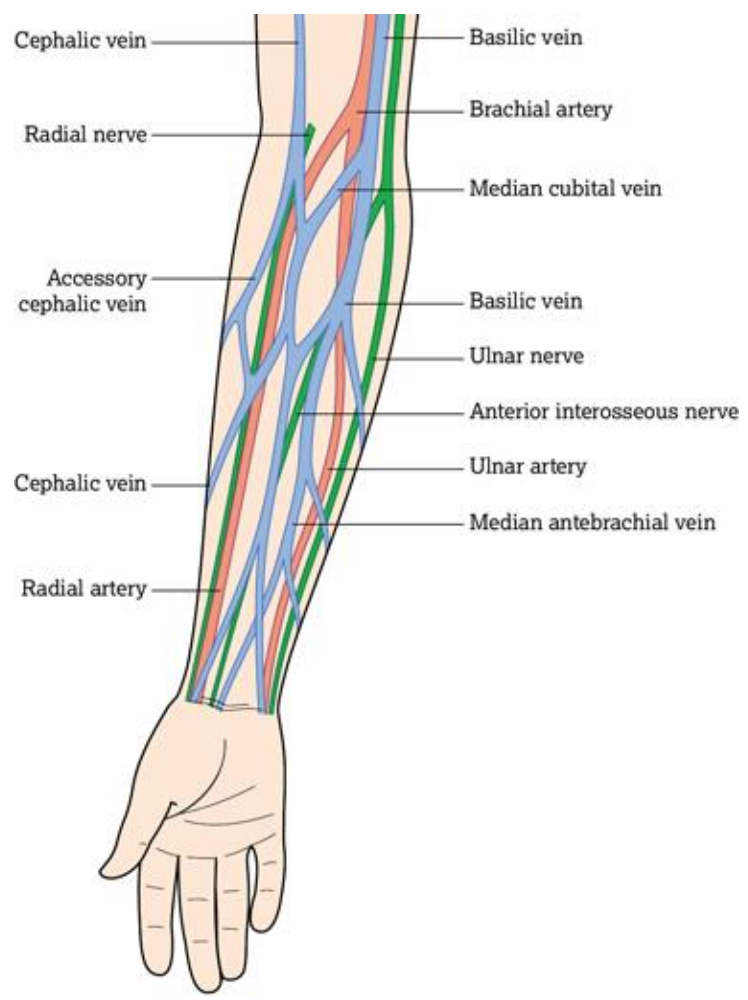

Figure 5.13 The vasculature of the forearm. Image from [125]. 


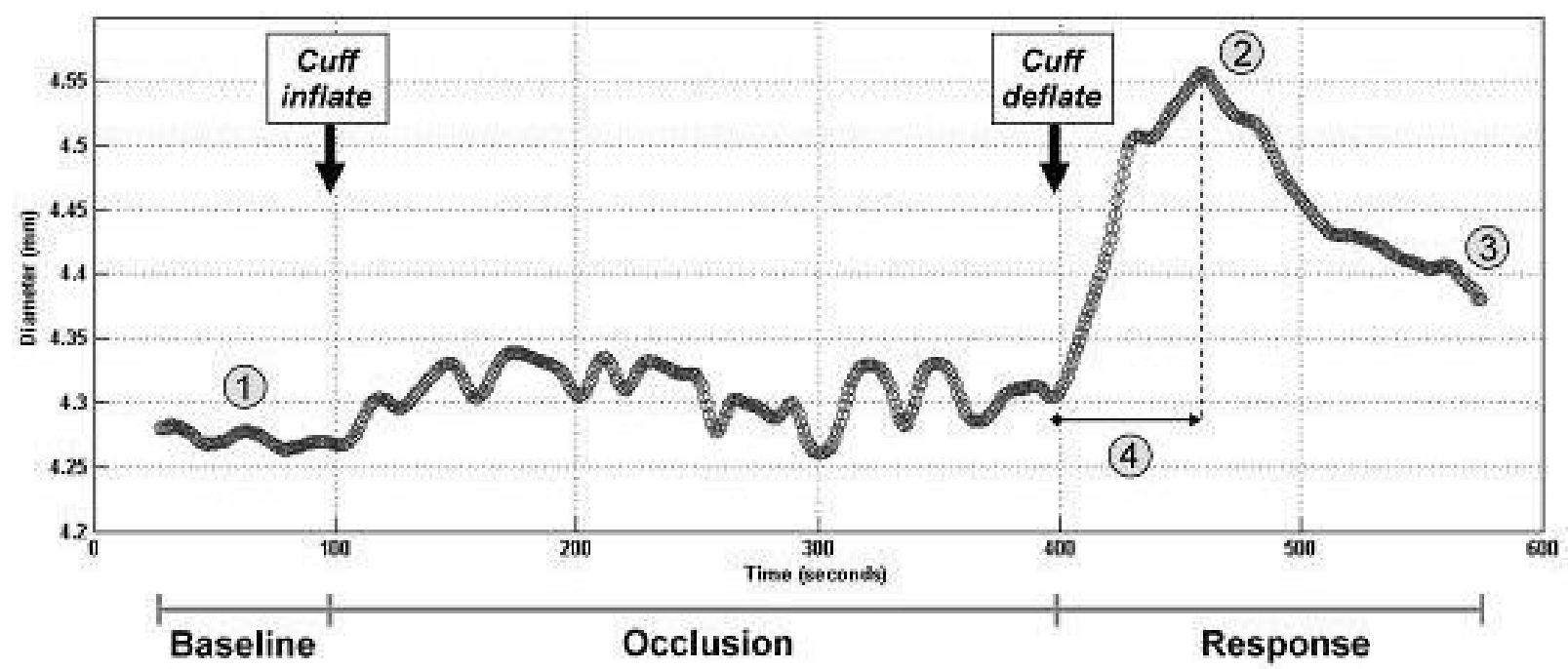

Figure 5.14 A subject's brachial arterial diameter in response to an operating blood pressure cuff as measured by a HDI 5000 Ultrasound Instrument, where these images were quantified using image segmentation. Image from [126].

All image segmentation methods were successful in segmenting the vessels of interest and quantifying the blood flow behaviour in response to blood occlusion. The basic method was the least sophisticated and the results parallel this; the regions are sensitive to intensity alone and so were not the best performer in identifying blood vessel size and shape. The watershed and DRLSE methods are both advanced algorithms and better identified vessel size and shape. The DRLSE method best defined the shape of each region, which is not surprising as the algorithm was created to maintain a desired shape [124]. The watershed method often identified non-vascular regions, resulting in messy segmentation, as compared to the other methods. The watershed algorithm segments the binary image into several different regions; here, the algorithmic system did not distinguish between labeled regions, instead, counted all labeled regions as one. In future work, this approach can be altered, which would likely improve the segmentation 'noise'. 
In examining the segmentation results over time, the basic thresholding, DRSLE and watershed methods reflect the expected pattern; the identified median cubital vein does not change in size or shape, while the identified ulnar artery shrinks in size leading up to 90 seconds, after which begins to grow, in some cases surpassing the initial artery area. This pattern coincides with the physical phenomenon of blood occlusion and consequent vascular recovery. Despite the region noise in the watershed segmentation results, this method revealed an area measurement pattern best describing the vascular behavior of blood occlusion over the specified time frame. The blood pressure cuff reached a maximum pressure exertion at approximately 80 seconds and started to deflate at approximately 85 seconds. The watershed region area results begin to decrease as the blood pressure cuff inflates and reaches a minimum area around 90 seconds, after which the area returns to original size. The DRLSE method resulted in smoother segmentations of the desired shape, but there are more variations in area measurement, perhaps due to the inclusion of larger extraneous objects in region detection. This will be addressed in future work by refining the DRSLE parameters and adding morphological operators post contour calculation.

In determining the best of the examined methods in the identification and segmentation of blood vessels and the monitoring of vascular behaviour in response to blood occlusion, the processing time should also be considered. The basic method was the least computationally expensive, the watershed method was slightly more computationally expensive and the DRLSE method was very computationally expensive. Considering segmentation performance, the ability to depict the physical vascular phenomenon, and processing time, the watershed method performed the best overall of the examined methods. 
Vital signal extraction methods for pulse measurements are detailed in Chapter 7. This includes details on the patient's pulse estimation over time from within the regions of interest defined in this chapter.

\subsection{Respiration Measurements}

This thesis moves towards more consistent and accurate respiration measurements using thermal cameras and spatio-temporal video processing. The extraction of normal respiration, and respiration abnormalities are examined in this thesis. While there are many data patterns associated with different respective patient morbidities, this thesis focuses on abnormal breathing associated with sleep deficiency. This section details the thermal infrared segmentation methods used to identify the regions of interest in human subjects for extraction of respiration signals. Visible-light video was not examined in relation to subject respiration measurements. The experimental conditions, set-up and procedures for subject data collection are described in Chapter 3.

For respiration measurement experimentation, fourteen subjects were recruited to perform a series of breathing tests. Each subject performed normal respiration, and then interrupted respiration with breathing irregularities, including hyperventilation, ceasing to breathe, and the absence of breathing in the presence of chest movements indicative of breathing. The extraction of normal respiration was first examined to determine whether thermal infrared and visible light cameras, in conjunction with video processing, can detect respiration. This was analyzed by creating five binary masks to specify ROIs. Two ROIs surrounded each nostril tightly, another two were larger to contain each nostril and some surrounding area, and the fifth ROI contained a larger area including both nostrils. These ROIs are depicted in Figure 5.15. All extracted, region-specific respiration signals were accurate representations of actual respiration, however the smallest ROIs 
were slightly better representations of respiration due to larger amplitudes. Although these ROIs performed slightly better than the rest, the results suggest that the larger, fifth ROI is a sufficient region in the extraction of respiration measurement from a subject.

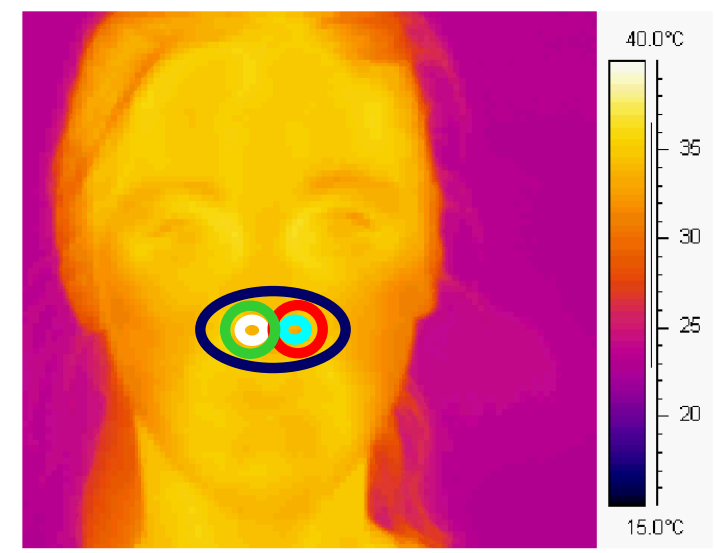

Figure 5.15 Five binary masks representing the ROIs.

The controlled experimentation of normal respiration and data analysis revealed that a subject's respiration measurements can be accurately extracted from a region enclosing the nose. In non-experimental environments, such as hospital environments, subjects move frequently. Region identification in the extraction of breathing signals was therefore further examined by implementing a facial recognition and nose identification algorithm.

Two facial recognition and region identification methods were tested by applying both methods to one thermal video of a subject and comparing the results. Method 1 began by calculating the image edges using the Prewitt method. A separate, binarized image was created by applying a grayscale threshold of 0.5 . The edge map image was added to the threshold binarized image by whole image pixel addition, resulting in a binary image better defining regions and edges of interest. This image was subjected to morphological operations to minimize details and segment the image into regions. The image was first subjected to morphological opening (erosion with a 
window of size $6 \times 6$, and then dilation with a window of size $7 \times 7$ ), in which the dilation was slightly more aggressive than the erosion, to encourage small regions in close proximity to merge. An example edged and thresholded image can be seen in contrast to the following morphologically opened image in Figure 5.16.

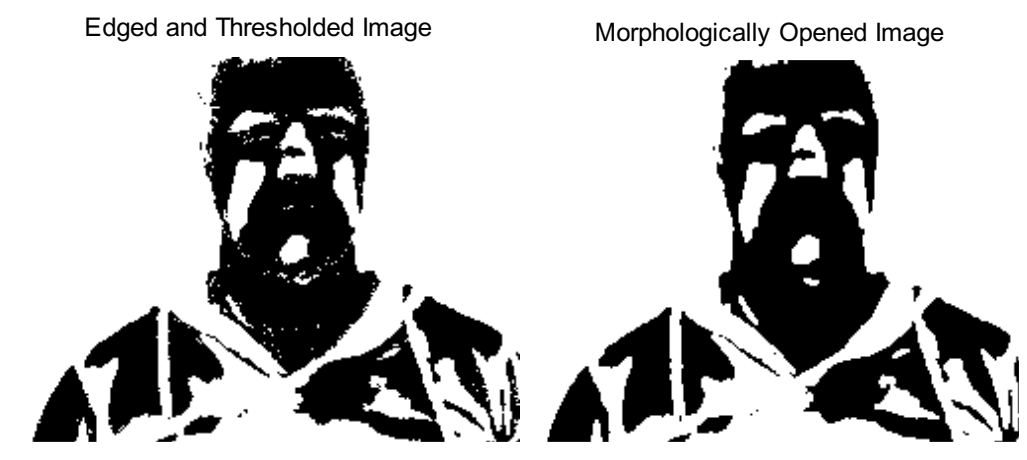

Figure 5.16 Image subjected to edge detection and thresholding, and morphological opening.

Connected components labeling (with an 8 connected neighbourhood) was applied, with the centroids of each separate component identified. K means clustering was applied to the connected component centroids, separating these data points into 3 clusters based on squared euclidean distance, using the best result from 10 replicates (each with different initial centroid positions). The largest cluster with the smallest sum of squared distances to the cluster centroid was defined as the face. The nose (the area just under the nose, including the nostrils) was located by first identifying the extrema of the face (the maximum and minimum values in the $\mathrm{x}$ and $\mathrm{y}$ directions of the facial cluster) and then the region closest to the center of the face. The extrema of the nose region was also calculated, and the ROI defined as a polygon of length spanning the width of the nose (from the left-top extrema to the right-top extrema), and polygon width half the length. An example of a connected components labeling image as compared to the nose identification can be seen in Figure 5.17. An example of the identified ROI can be seen in Figure 5.18. 


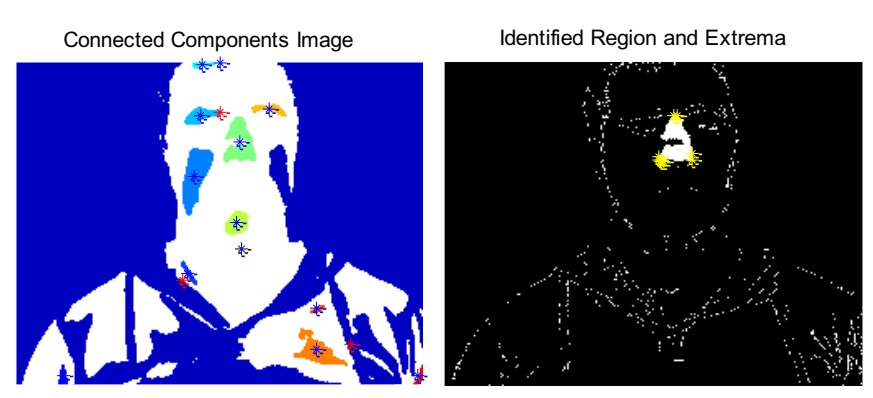

Figure 5.17 Image subjected to connected components labeling and region identification.

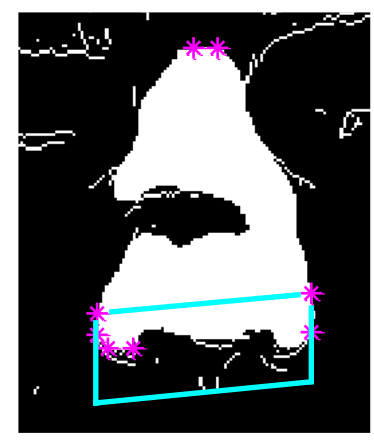

Figure 5.18 Identified region of interest.

The facial recognition and region identification method 2 performed different processing techniques on every frame of the 2150 frame thermal video to extract the same signal: respiration rate. Method 2 processing began by applying the Viola-Jones algorithm to each smoothed, enhanced thermal image. The Viola-Jones algorithm is used to find facial features in a grayscale visible-light image, which has different topographical properties than a grayscale thermal image [127]. However, when examining a subject's mouth, a visible-light image and thermal image complement have similar topographical properties. The Viola-Jones algorithm is therefore used here to find the location of the subject's mouth in each thermal image. The algorithm uses Haar feature selection, adaboost training and cascading classifiers to detect objects defined by an input character vector, which in this thesis was [15 25], representing a subject's mouth [127]. An example of a detected mouth in a thermal image complement can be seen in Figure 5.19. 


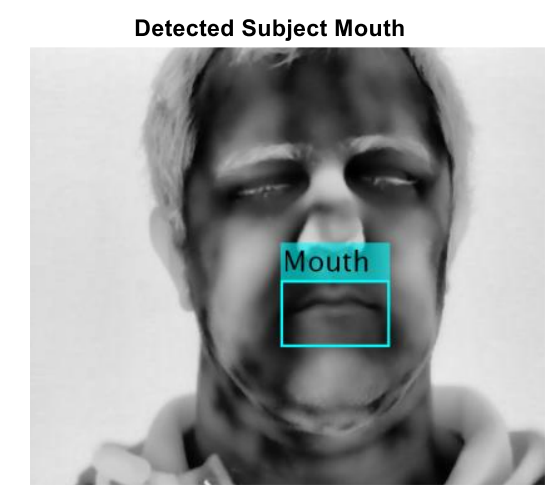

Figure 5.19 Identified subject mouth.

The image gradient (sobel algorithm) of the smoothed, enhanced images was also calculated. The gradient magnitude was then binarized using Otsu's method to determine a threshold. This binarized image was morphologically closed using a $3 \times 3$ window, to remove small image structures, leaving a gradient image edge map. For each image, the location of the mouth was used to define an area of interest; the gradient image edge map was used to locate the nose. The area of interest was defined as an image region as wide as the identified mouth, with a height spanning from the top edge of the mouth region to the top of the image. Each row of this binarized image area was summed and the maximum sum was defined as the nostrils. An example of a defined image area and the resulting summed pixel rows can be seen in Figure 5.20. 

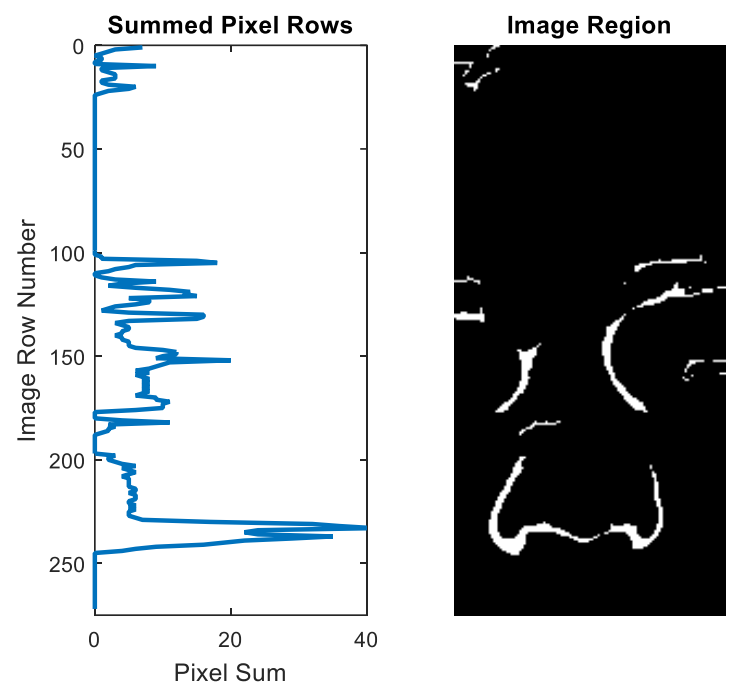

Figure 5.20 Depicted method of locating a subject's nostrils.

Each of 2150 thermal video frames were subjected to the described analyses. The image correction and enhancement stage of the algorithm successfully reduced noise and enhanced the image contrast, as was determined by examining the original thermal video for temperature changes over time. Both facial recognition and region identification methods optimized several parameters (subject dependent optimization) to successfully identify the region just under the nose for the majority of the thermal video. In contrast to Method 1, Method 2 resulted in better ROI identification (approximate 90\% verses 80\% accuracy) and a stronger respiratory signal.

Vital signal extraction methods for respiration measurements are detailed in Chapter 7. This includes details on the patient's respiration signal over time from within the regions of interest defined in this chapter.

\subsection{Temperature Measurements}

This thesis examines body temperature in human subjects; namely the relationship between temperature and pressure, how temperature can inform blood flow and perfusion, and the 
development of pressure ulcers. This section details thermal infrared image segmentation methods used to identify the regions of interest in human subjects for extraction of temperature measurements.

For temperature measurement experimentation, data from healthy adult volunteers performing controlled experiments was captured with the goal of first identifying and quantifying thermal changes occurring in response to applied pressure. Healthy adult experimentation is detailed in Chapter 3 and consisted of a subject applying pressure to their hands, then monitoring temperature changes over the following 10 minutes using a handheld thermal camera. Following image preprocessing described in Chapter 4, the thermal images were analyzed to identify regionspecific temperature patterns. Because the thermal camera was handheld, all the images taken were at slight angles, translations and scales when compared to the baseline image. Image registration was then performed to align all pressure recovery images to respective baseline images. An example of two aligned, overlaid images can be seen in Figure 5.21, where the purple tinted areas are one image and the green tinted areas are the second image.

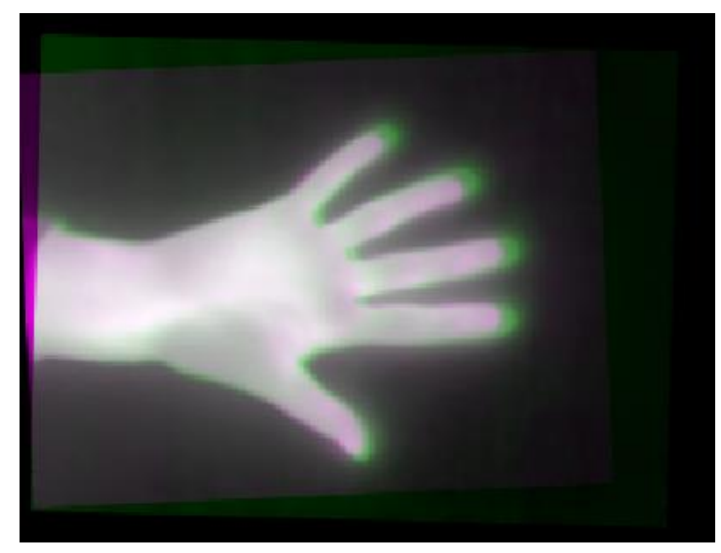

Figure 5.21 Two overlaid registered images. 
Region-based temperature measurements were then extracted to illustrate pressure induced thermal changes in skin over time. The first measurement was the area of respective temperature grades. Like histogram calculations, the pixel values were separated into 15 levels (or bins), representing each $1.0^{\circ} \mathrm{C}$ increment in the thermal image temperature range $\left(20.0^{\circ} \mathrm{C}\right.$ to $\left.35.0^{\circ} \mathrm{C}\right)$. Contours separating temperatures represented by pixel value were found; an example of which can be seen in Figure 5.22. Each contour encloses an area; these areas were calculated for each contour line. Areas were calculated based on unitless pixel number and represent the number of pixels at respective temperature levels.

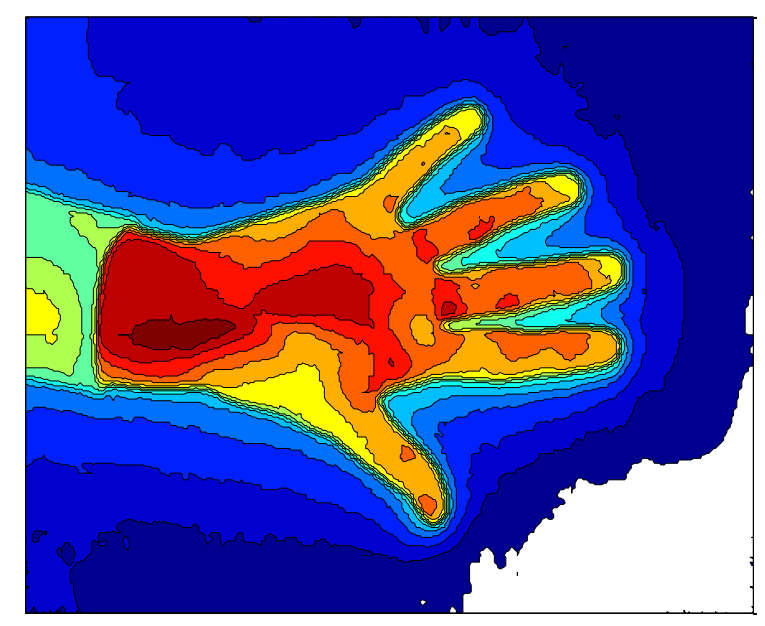

Figure 5.22 Contour plot at specified levels.

Another binary image mask was created to specify the ROIs illustrated in Figure 5.23, where each color represents a different ROI. The regions were chosen to include the area assumed to endure the highest pressure (the palm) and the individual areas assumed to endure less pressure (fingers and wrist). Intensity measurements were extracted from the illustrated ROIs of each image. 


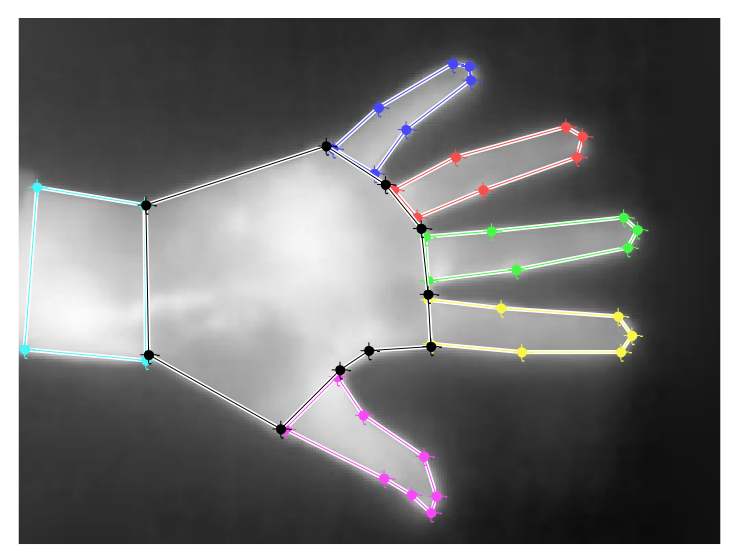

Figure 5.23 Depiction of ROIs.

The contour plots yielded a visually identifiable thermal pattern. This pattern is illustrated in Figure 5.24. The first subplot illustrates the hand before applying any pressure. The second subplot illustrates the hand directly after applying heavy pressure for five minutes. The third subplot illustrates the hand five minutes after applying heavy pressure and the last image illustrates the hand ten minutes after applying heavy pressure. The temperature pattern is visually identifiable; the hand initially cools at the areas of direct contact (fingers and base of the palm), then the whole hand warms over time to temperatures exceeding that of the baseline image. The area contours over time were quantified and are presented in Chapter 9. 

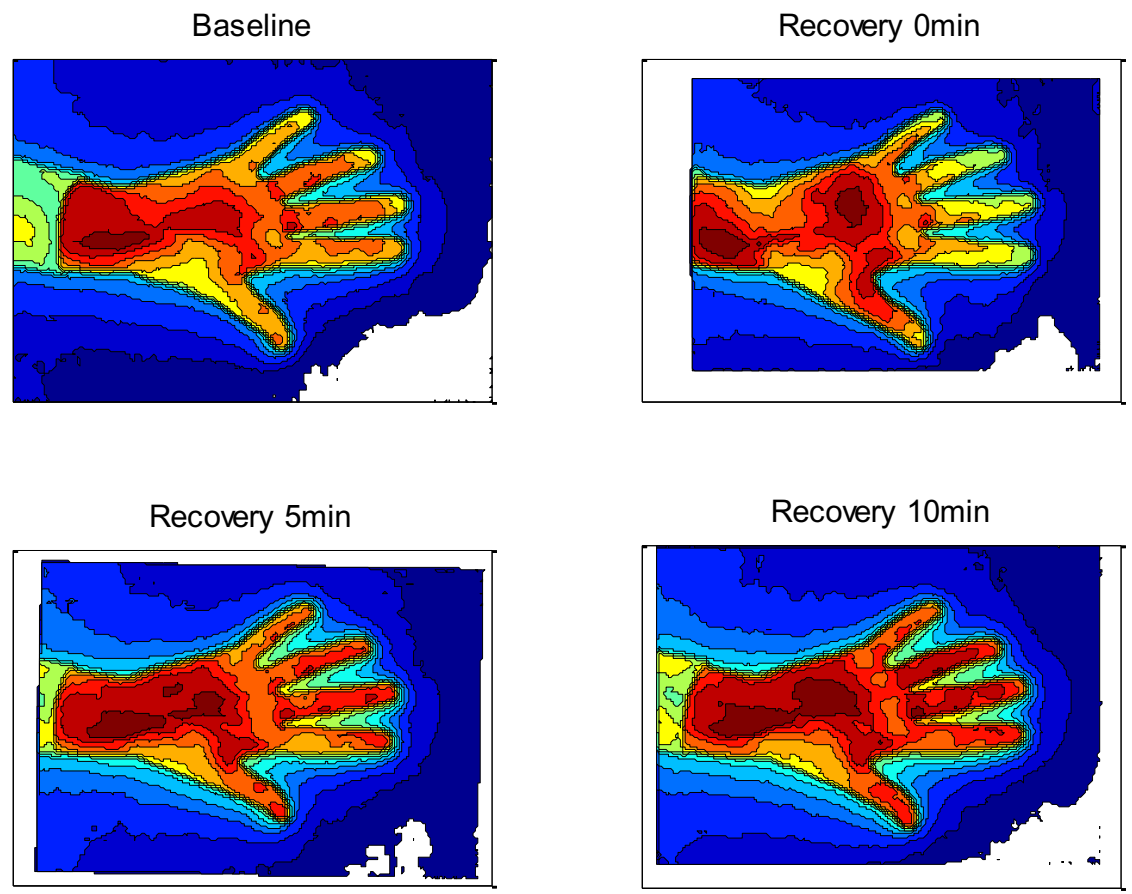

Figure 5.24 Contour plots of the baseline and three recovery images following the application of heavy pressure.

Thermal imagery of healthy adult subject hands was examined in order to establish possible thermal patterns due to applied pressure. The resulting knowledge was applied to data capture of older adult in-hospital patients, with the intent to gain knowledge about the development of pressure ulcers in a hospital setting. Data collection procedures from older adult patients is detailed in Chapter 4 and consisted of capturing thermal images of eight patient's heels and malleoli once a week for several months. The timeline varied based on the individual patient, for example any given patient could be moved to acute care if their condition deteriorated at any given time. In addition to thermal data capture, pressure data was captured on a continuous basis. Pressure mats were placed underneath the patient's mattress and collected data 24 hours a day, 7 days a week. The thermal images were examined over one night to determine temperature patterns; temperature distributions of the left heel compared to the right heel. These thermal patterns were cross 
examined with the pressure measurements to observe possible relationships between pressure and temperature distribution (presented in Section 5.5). Thermal images were then examined for one patient over the entire duration of their stay, to observe the temperature measurements over time.

The thermal images of the patient were examined through contours. A total of 9 contours were calculated, each separating one degree in temperature between $23^{\circ} \mathrm{C}$ and $32^{\circ} \mathrm{C}$. Figure 5.25 depicts a grayscale image of the patient's left heel and the resulting contour plot.
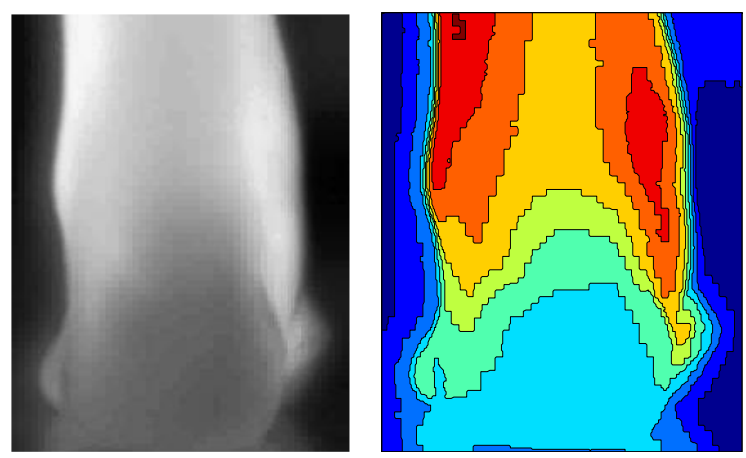

Figure 5.25 Grayscale image of the left heel and the resulting contour plot.

Each contour line encloses a surface area and each area represents the amount of skin at respective temperatures. These areas were calculated in terms of pixels; each area representing the number of pixels at respective temperature levels. Area ratios of the left heel compared to the right heel were calculated as L/R. These measures of comparison were considered in addition to absolute measures to best discount the effects of external stimuli.

Thermal images were analyzed through measurements of comparison to make thermal observations while minimizing consideration of external thermal influences. Measures of comparison are also important in establishing baseline values for subjects. In general, healthy feet may vary drastically in temperature distribution from subject to subject, but are far less likely to 
have large differences between their own feet. Asymmetry can indicate blood flow issues including inflammation or blood occlusion, among other causes.

Thermal image contour plots of both the patient's feet and a healthy adult's feet can be seen in Figure 5.26 and Figure 5.27, respectively. There is a large, visible difference in patient heel temperatures; the lower left heel is a cooler temperature when compared to the right heel. Contrary to patient results, the healthy adult heel temperatures are visibly similar. The ratios of areas at each temperature level for both patient and healthy heels can be seen in Table I and confirmed visually identifiable patterns. The patient's left heel was colder than the right and $\mathrm{L} / \mathrm{R}$ ratios varied (in the patient, heel-specific temperature range of $26^{\circ} \mathrm{C}$ to $29^{\circ} \mathrm{C}$ ) from 0.29 to 3.67 . This is a wide range when compared to the healthy heel ratios (in the healthy, heel-specific temperature range of $24^{\circ} \mathrm{C}$ to $30^{\circ} \mathrm{C}$ ) varying from 0.77 to 1.84 .
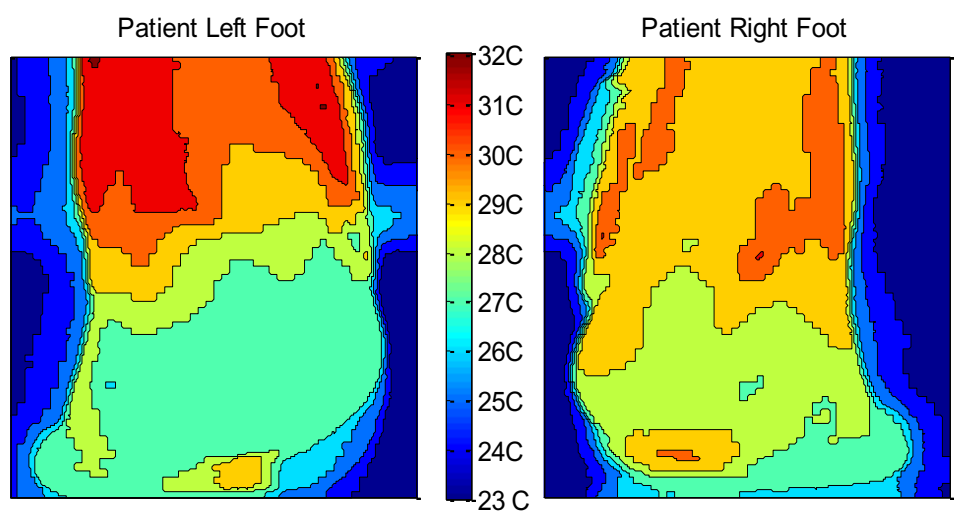

Figure 5.26 Contour plots of the patient heels. 


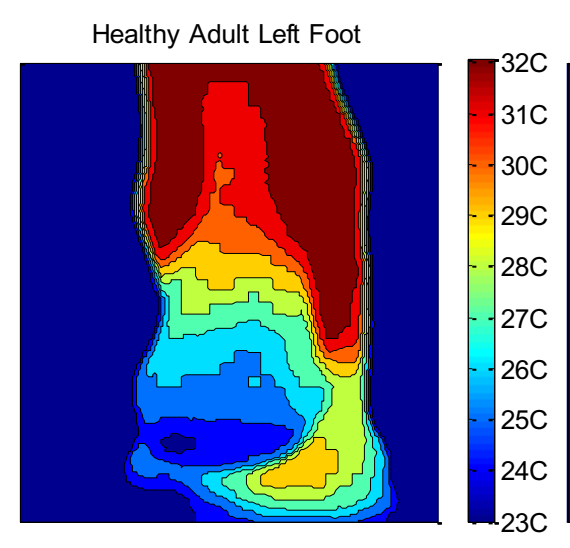

Healthy Adult Right Foot

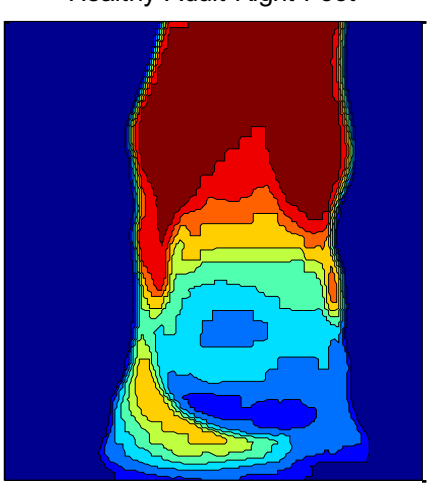

Figure 5.27 Contour plots of a healthy adult's heels.

Table 5.1 Heel Temperature Level Pixel Areas

\begin{tabular}{|c|c|c|c|c|c|c|}
\hline \multirow{2}{*}{$\begin{array}{c}\text { Temp } \\
\left({ }^{\circ} \mathbf{C}\right)\end{array}$} & \multicolumn{3}{|c|}{ Patient Pixel Areas } & \multicolumn{3}{c|}{ Healthy Adult Pixel Areas } \\
\cline { 2 - 7 } & $\begin{array}{c}\text { Left } \\
\text { Heel }\end{array}$ & $\begin{array}{c}\text { Right } \\
\text { Heel }\end{array}$ & $\begin{array}{c}\text { Ratio } \\
\text { L/R }\end{array}$ & $\begin{array}{c}\text { Left } \\
\text { Heel }\end{array}$ & $\begin{array}{c}\text { Right } \\
\text { Heel }\end{array}$ & $\begin{array}{c}\text { Ratio } \\
\text { L/R }\end{array}$ \\
\hline 23 & 5401 & 9268 & $\mathbf{0 . 5 8}$ & 9018 & 9315 & $\mathbf{0 . 9 7}$ \\
\hline 24 & 3535 & 3339 & $\mathbf{1 . 0 6}$ & 756 & 410 & $\mathbf{1 . 8 4}$ \\
\hline 25 & 4727 & 2914 & $\mathbf{1 . 4 6}$ & 1412 & 1685 & $\mathbf{0 . 8 4}$ \\
\hline 26 & 1918 & 2108 & $\mathbf{0 . 9 1}$ & 1149 & 1497 & $\mathbf{0 . 7 7}$ \\
\hline 27 & 15456 & 4206 & $\mathbf{3 . 6 7}$ & 1001 & 981 & $\mathbf{1 . 0 2}$ \\
\hline 28 & 5038 & 10272 & $\mathbf{0 . 4 9}$ & 934 & 608 & $\mathbf{1 . 5 4}$ \\
\hline 29 & 4477 & 15532 & $\mathbf{0 . 2 9}$ & 761 & 749 & $\mathbf{1 . 0 2}$ \\
\hline 30 & 5733 & 4346 & $\mathbf{1 . 3 2}$ & 567 & 453 & $\mathbf{1 . 2 5}$ \\
\hline 31 & 5471 & 15 & $\mathbf{3 6 4 . 7}$ & 1110 & 706 & $\mathbf{1 . 5 7}$ \\
\hline 32 & 244 & 0 & $\mathbf{0}$ & 2922 & 3226 & $\mathbf{0 . 9 1}$ \\
\hline
\end{tabular}

When comparing results of the left heel to the right heel, it is interesting to note that the left heel was colder, while also having experienced more applied pressure and less movement (more details can be found in the following chapters). While many factors contribute to the temperature distribution of the feet, namely health related impedance of blood flow to the extremities, a patient's in-bed behavior may also contribute. Considering the amount of time an at-risk elderly patient spends in bed (often sleeping), and the known effects of prolonged pressure 
on susceptible tissue, it is reasonable to consider that the temperature distribution of the feet may be related to a patient's long term sleeping behavior.

Long term thermal data was examined for one 92-year-old female, who began development of a pressure ulcer over a duration of 112 days. The patient began complaining of pain in her right heel on the 6th day of the study and appeared to experience some pain relief by the 106th day of the study. The patient was not accessible between days 70 to 100 . As detailed in Chapter 3, a researcher visited the hospital once a week to gather thermal image data. Data collection occurred at the same time every visit. The researcher would take images of the right heel, right lateral malleolus, left heel and left lateral malleolus. An image of the room was also collected for ambient temperature measurement.

Following image preprocessing, detailed in Chapter 4, a region of interest was identified for each thermal image. These regions were placed manually, as the images were taken on different days, of a patient in varying positions (sitting verses lying), and therefore one image is often at differing translations and rotations respective to another. Image registration was not used to maintain image integrity as the camera angles were very different from day to day. Each region was defined as an ellipse and placed over the areas of interest; the right and left heels and lateral malleoli. An example of region selection for each group of images can be seen in Figure 5.28. For each data collection day, and each anatomical landmark (heel, malleoli) intensity measurements were extracted and compared between the left ROI and the right ROI. 

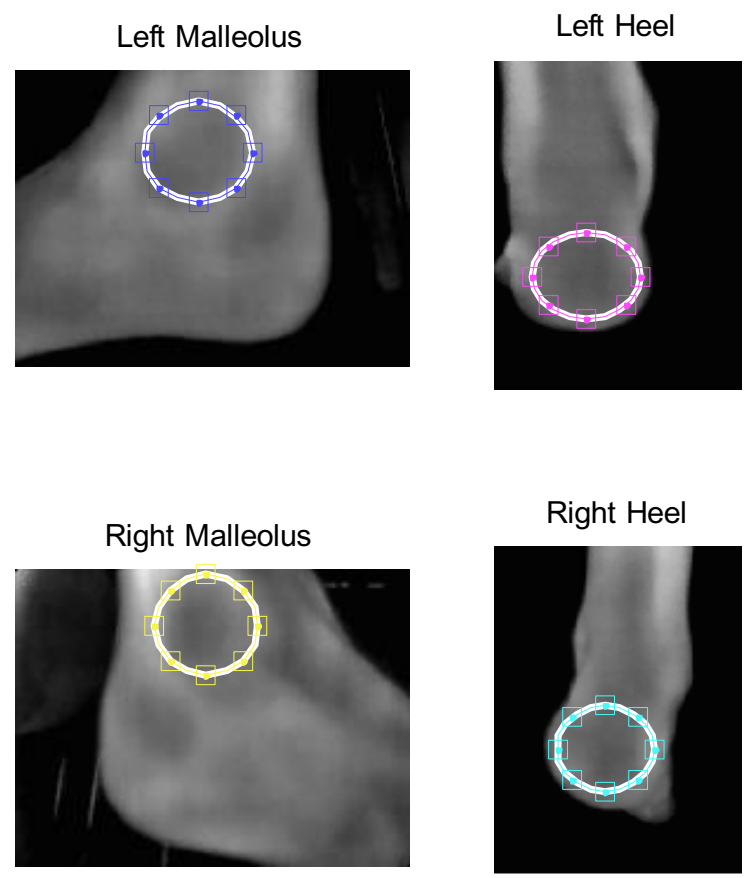

Right Heel

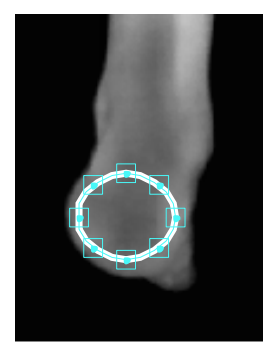

Figure 5.28 Regions of interest from each anatomical position.

Large regions of interest were favored over small regions; the nature of hospital data collection means that small changes in temperature could be the result of any number of external factor and are therefore unreliable. Considering larger regions and averaging temperatures within regions is more suitable for this application as the patterns are more reliable. These averaged, larger temperature regions were successful in indicating a distinct temperature pattern that is consistent with the physical phenomenon of a stage 1 pressure ulcer as it developed and was treated.

Vital signal extraction methods for temperature measurements are detailed in Chapter 7. This includes details on the patient's temperature signal over time from within the regions of interest defined in this chapter. 


\subsection{Mobility Measurements}

This thesis examines the use of pressure sensors in the extraction of mobility measurements. Specifically, this thesis explores the center of pressure as a mobility measure (COP) as well as the relationship between pressure and temperature. This section describes the spatial locations from which pressure measurements were extracted.

For mobility measurement experimentation, data from healthy adult volunteers performing controlled experiments was captured in the examination of COP. One hospital bed was equipped with three pressure sensitive mats. Two young, healthy, female non-patient volunteers were recruited to perform a series of movements on the hospital bed that constituted four continuous data sets. Each subject began in a lying position, rolled to each side of the body, then transitioned from lying, to sitting, to a final standing position. Equipment set-up and experimentation are described in more detail in Chapter 3. The central mobility measurement, center of pressure (COP), is a spatial location, so region identification and mobility measurement extraction are one and the same for this vital signal. The COP algorithms are presented in Chapter 9.

Mobility measurements were also captured of patients susceptible to the development of pressure ulcers in a hospital setting. The experimentation is detailed in Chapter 4 and consisted of capturing pressure data on a continuous basis. Pressure mats were placed underneath each of eight patient's mattresses and collected data 24 hours a day, 7 days a week. In addition to pressure data, thermal images were captured of patient's heels and malleoli once a week for several months. The timeline varied based on the individual patient, for example any given patient may be moved at any given time to a different hospital. Data was examined over one night for one patient to observe possible relationships between pressure and temperature distribution (presented also in Section 
5.4). The night was considered inclusive of the time frame between 9pm and 8am; hours chosen based on hospital observation and staff schedules. Pressure patterns resulting from the mat situated under the patient's feet were examined during these hours. The regions of interest for mobility measurements in this thesis are the entire pressure mat area situated under each subject's feet. The mobility measurement algorithms are presented in Chapter 9.

\subsection{Conclusion}

Pre-processed data, the algorithms for which are detailed in Chapter 4, were used as an input to this stage of data proccesing; data segmentation. Segmentation is an important part of vital signal measuerment, as vital signals are generally measured from specific areas of the body. If these are not identified correctly, the resulting measurements will be incorrect. This chapter details the methods used to identify regions corresponding to respective vital signals. The resulting vital signal measurements are detailed in chapters 7-9.

Regions of interest for pulse measurement extraction were identified through several methods. Proximal pulse regions were identified in both visible-light and thermal-infrared video using binary masks placed at anatomical locations known to reveal heart rate; i.e. over major blood vessels, or areas successfully used by numerous previous studies (forehead). Distal pulse regions were considered to be regions containing a subject's arm or leg, and not the subject's chest or face. Subject feet were examined, and anatomical regions of interest were identified in both visible light and thermal infrared images using binary masks. Subject arms were also examined via thermal infrared cameras. Anatomical regions of interest were identifed using three different methods of image segementation; basic thresholding, watershed method and level set method. Watershed 
method best segmented blood vessels of interest and portrayed the blood flow patterns of vascular occlusion and relief.

Respiration was performed by subjects through the nose, and examined through thermal video data. Regions of interest were therefore considered to be the area surrounding a subject's nostrils. These regions were identified using binary masks, as well as two methods that include region tracking, and therefore account for subject motion. The first method was based on connected components analysis, and the second method was based on the Viola-Jones algorithm. Method 1 resulted in an $80 \%$ region identification accuracy, whereas Method 2 resulted in a $90 \%$ region identification accuracy, and a stronger respiratory signal.

Temperature in this thesis is examined in reference to mobility measurements and as temperature and mobility measurements relate to and inform pulse measurements. Temperature was examined in subject extremities through thermal image data; this included images of subject hands and feet. These images were segmented using binary masks to identify regions of interest, and using contours to segment the image by temperature. These techniques were able to reveal temporal temperature patterns related to applied pressure and consequent changes in blood flow.

Data segmentation in pressure mat data consisted of identifying regions spatially that were relevant to mobility measurements. These regions included the entire pressure mat directly under subject's feet, as well as a center of pressure measurement to attain an overall measurement of spatial movement.

This chapter demonstrated the importance of data segmentation in the extraction of vital signals. All data segmentation methods detailed in this chapter successfully identified regions of interest for extraction of respective vital signal measurements. The results from this section are 
described here and consolidated quantitatively with the extracted vital signal measurement results detailed in chapters 7-9. 


\section{Chapter 6}

\section{Video Magnification in Vital Signal Extraction}

This chapter details the development of video processing algorithms used in this thesis as well as parameter testing and application of these algorithms, prior to vital signal extraction detailed in Chapter 7.

\subsection{Introduction}

Spatio-temporal processing applied to video data to extract biosignals is an emerging technique

[9]. A popular spatio-temporal processing technique is called Eulerian Video Magnification. Eulerian Video Magnification (EVM) is a procedure developed by the Massachusetts Institute of Technology (MIT) that works towards device versatility by allowing a device as small and widely available as a smart phone to measure biological signals by simply capturing video footage of a subject [9]. This procedure breaks a video down spatially and temporally to amplify variations occurring within a specified frequency. The result is the enhancement of motion or color variations that cannot be seen by the human eye. Research has shown this procedure (and similar methods) can use visible light video footage to reveal biological signals including adult heart rate and respiratory rate, infant heart rate and respiratory rate, pulse transit time, finger vein liveness detection, among others [9],[66],[67],[128]. Recently, EVM has been examined in relation to thermal video. Thermal video in conjunction with EVM and post-processing (signal extraction), has been shown to successfully reveal respiration rates and respiration patterns indicative of 
obstructive sleep apnea [21]. Thermal cameras have the potential to reveal biological signals that regular cameras may not be able to. For example, the effects of pressure on blood flow can be seen using a thermal camera but may not be revealed using regular video [24]. This thesis examines the extraction of vital signals from three different modalities, with an emphasis on the extraction of respiration and pulse from thermal cameras.

While EVM has been shown to successfully and unobtrusively enhance biological signals, the drawback of EVM is that it can significantly amplify noise as the magnification factor increases [10],[11]. This, coupled with the inherent noise occurring during regular and thermal video acquisition leads to enhancement issues. The use of EVM with a narrow bandpass filter can lead to the amplification of noise alone and the false attribution of this signal to blood flow and heart rate. An algorithm was developed for this thesis that aims to mitigate this effect using an adaptive EVM approach. This approach first employs a wide bandpass filter with a low amplification factor to identify an underlying signal, then re-processes, employing a narrow bandpass filter with a higher amplification factor to reveal a biological signal, not amplified noise.

This chapter details the Eulerian Video Magnification method as well as the Adaptive Eulerian Video Magnification (AEVM) developed in this thesis. Additionally, this chapter tests the EVM algorithm as well as the developed AEVM algorithms for robustness with respect to noise. This chapter details the stage of processing (within the structure of the thesis) depicted in Figure 6.1. Results are presented in the following sections for both subjects and for simulations. 


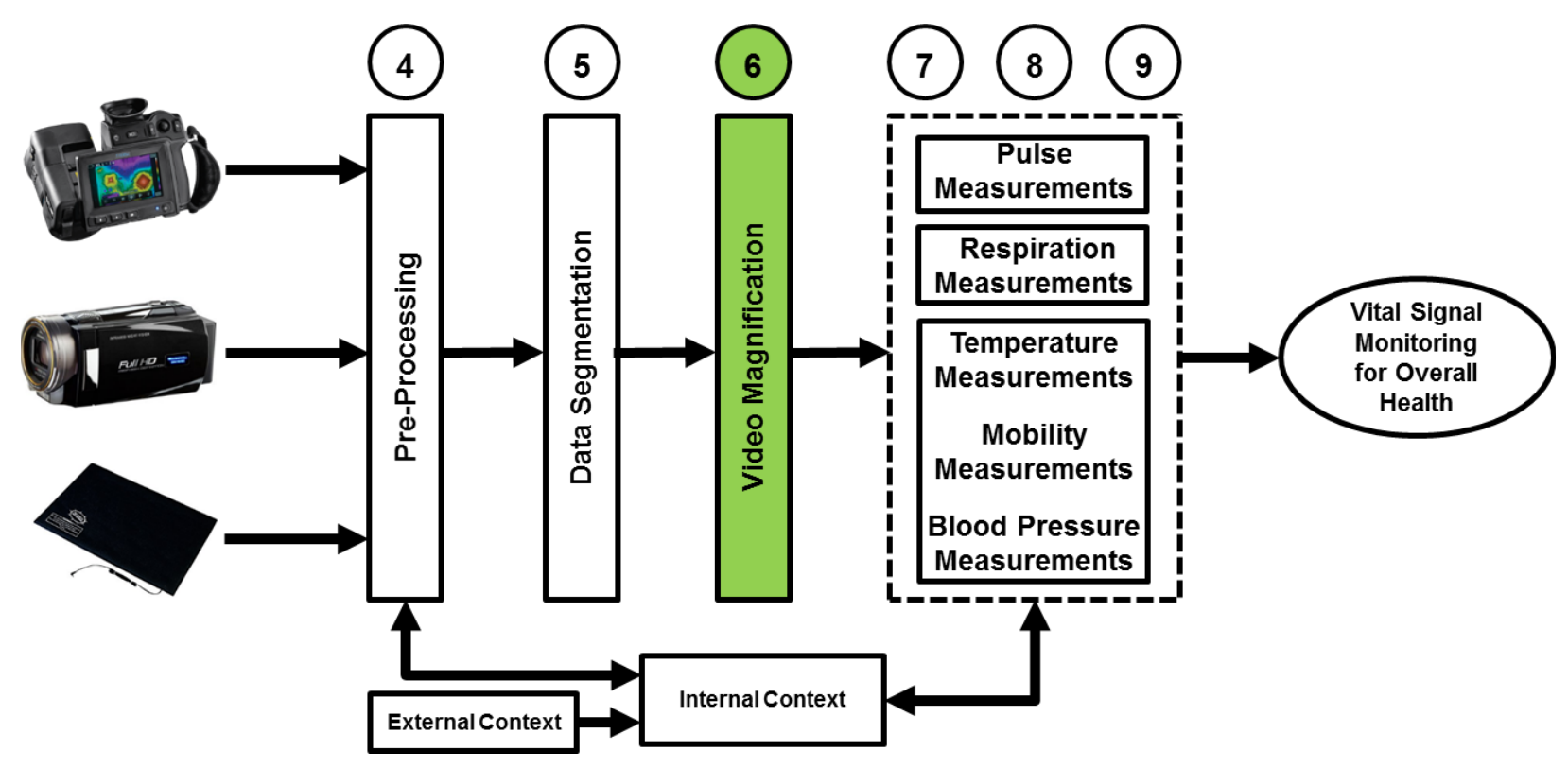

Figure 6.1 Video magnification stage of the thesis structure

\subsection{Eulerian Video Magnification}

Eulerian video magnification has been used extensively on visible light video, as detailed in Chapter 2. The procedure breaks each frame of a video into pixels, determines its color (or intensity) value, and amplifies temporal variations occurring within a specified frequency band. The following provides an overview of the Eulerian enhancement procedure, which is depicted in Figure 6.2 (image from [9]).

This thesis performs EVM processing in both the RGB and YIQ color spaces. In the YIQ color space, $\mathrm{Y}$ represents luminance and I and Q represent chrominance information. Chrominance represents colour information separate from the greyscale luminance information. Each frame of each RGB or YIQ video is first spatially filtered using Gaussian pyramid decomposition [66]. Pyramid decomposition consists of repeated smoothing (with a specified kernel) and subsampling. The EVM method performs smoothing with a user specified kernel, then down-samples by a factor 
of 2 in both the $\mathrm{x}$ and $\mathrm{y}$ directions. This process is repeated a user specified number of times; a parameter referred to as the pyramid level number. The default parameters are a binomial kernel (window of size 5) and 4 levels of spatial filtering [71].

Following pyramid decomposition, the EVM algorithm performs FFT on each pixel within each color channel, zeroes out components outside the user specified frequency band and then performs IFFT to return the signal to the time domain [71]. The signals from the chrominance and luminance channels were then amplified using separate amplification factors. The amplified signal is added back to the original signal and the spatial pyramid is then collapsed, resulting in the desired output video.

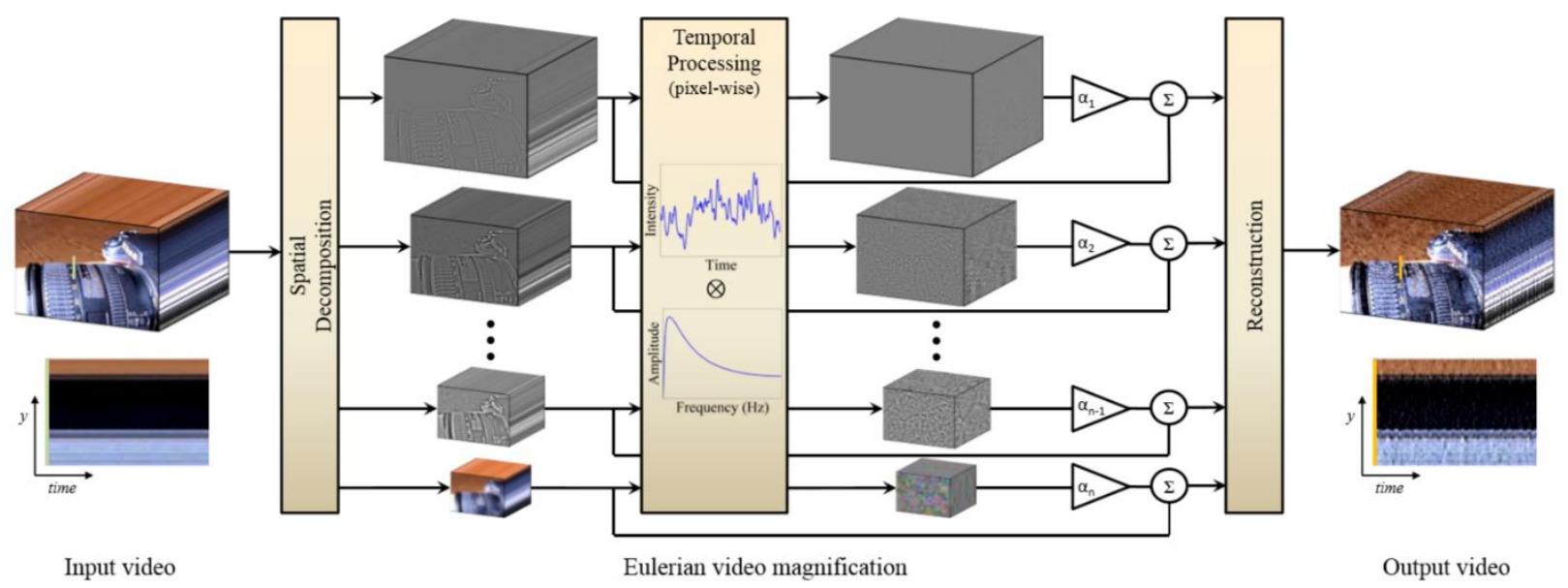

Figure 6.2 Eulerian video processing steps (image from [9]).

The EVM method can be used for motion amplification as well as color amplification and as such, is customizable to the user's needs. The user specifies several parameters including the filter type, the spatial level, the frequency band of interest and the magnification factors.

The use of a narrow bandpass filter in the EVM algorithms can lead to the amplification of noise, falsely identified as an enhanced biosignal. Due to inherent noise in the thermal and light 
videos (occurring during acquisition), EVM, using a narrow bandpass filter can reveal temporal color variations that do not represent heart rate. An example of this is depicted in Figure 6.3. Thermal video, visible light video and ECG data were captured of a subject sitting quietly. The light and thermal videos were subjected to EVM using a narrow bandpass filter with cut-off frequencies $\mathrm{f}_{\text {low }}=1.33 \mathrm{~Hz}$ ( 80 beats per minute), $\mathrm{f}_{\text {high }}=1.50 \mathrm{~Hz}$ ( 90 beats per minute) and an amplification factor of 100 . The light and thermal video mean intensity signals depict the same average frequency of $\mathrm{f}=1.43 \mathrm{~Hz}$ ( 86 beats per minute), whereas the ECG signal reveals that the actual heart rate is at a frequency of $\mathrm{f}=0.88 \mathrm{~Hz}$ ( 53 beats per minute).

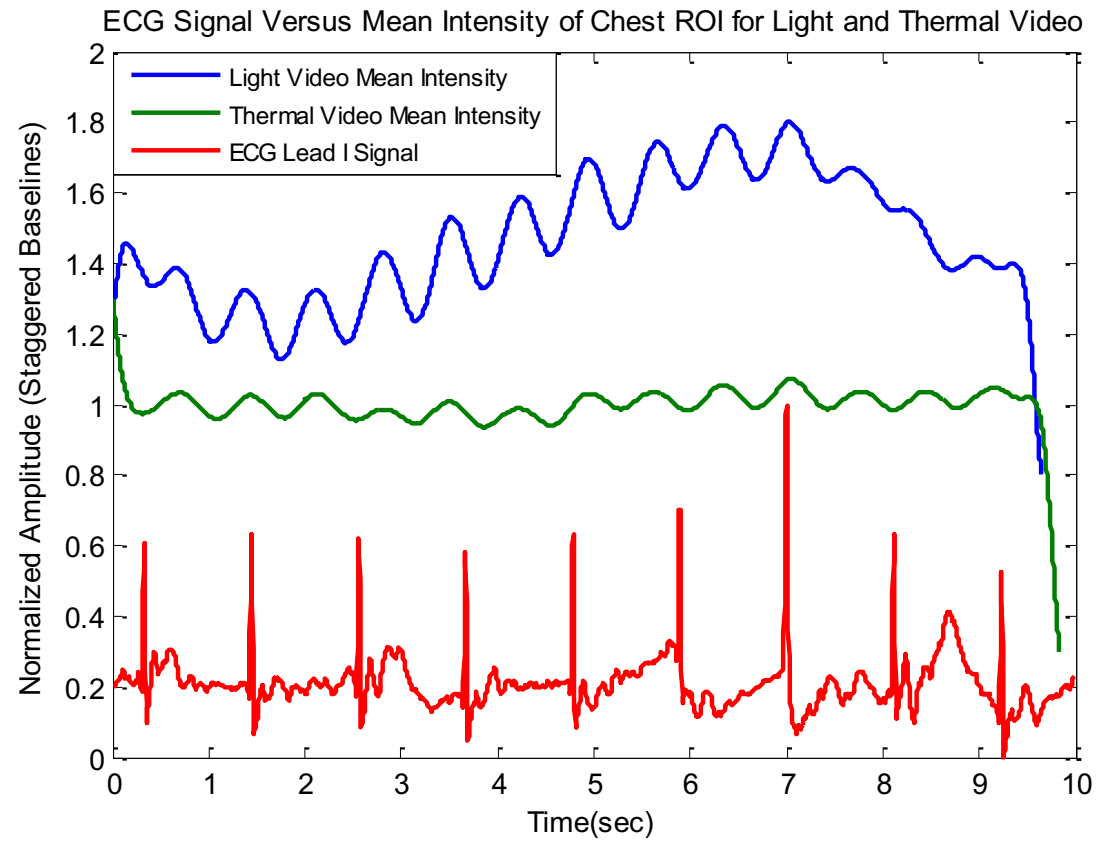

Figure 6.3 Extracted vital signals from visible light and thermal infrared video (EVM bandpass filter $1.33 \mathrm{~Hz}$ to $1.5 \mathrm{~Hz}$ ) versus ECG signal.

The following section describes the adaptive Eulerian video magnification method proposed to mitigate this problem, which is utilized in the following thesis chapters. 


\subsection{Adaptive Eulerian Video Magnification}

Eulerian video magnification can be used with video footage from any modality, and therefore has the potential to extract a variety of biological signals. However, due of the presence of noise in video data, EVM for color (or intensity) amplification using a narrow bandpass filter (suggested in [9] for best results) results in an amplified signal within any narrow passband. This means one can falsely report a biological signal. This thesis looks to mitigate this problem by introducing an adaptive EVM method, and focuses on the use of AEVM on thermal video to extract pulse and respiration measurements. While both pulse and respiration measurements are examined in this thesis, this chapter details the methods of AEVM applied to thermal video of one subject as a proof of concept, then presents aggregate results of several subjects in the vital signal extraction chapter (Chapter 6).

Data was gathered from one, healthy adult volunteer. All equipment used for experimentation is discussed in further detail in Chapter 3. Following a 15 minute equilibrium period, the subject put on a Hexoskin smart textile shirt and ensured the two textile sensors (at the thoracic and abdomen areas) were in contact with the skin. The thermal camera and visible light camera were set-up to face the subject. The equipment start times were staggered due to the manual nature of the laptop and iPad. This was accounted for by having the subject grab the thoracic textile band and move it in a vigorous manner. This introduced noise in the ECG signal and a simultaneous visual cue in the thermal and light video recordings. Following this movement, the subject was still for approximately one and half minutes. This section uses ten seconds of data. The subject sat in a chair, as still as possible, throughout experimentation. This was important, as subject motion can affect EVM results. 
Data processing began by subjecting the visible light video data to EVM processing, using a wide passband and a relatively low magnification factor (all EVM parameter values are specified in Table 6.1). The enhanced output video was then examined to see if the correct heart rate signal could be extracted despite the use of a wide passband.

Table 6.1 EVM Parameter Values for Wide Passband

\begin{tabular}{|c|c|c|}
\hline Parameter & Light Video & Thermal Video \\
\hline $\begin{array}{c}\text { Low frequency bound of } \\
\text { the band-pass filter }\end{array}$ & $0.5 \mathrm{~Hz}$ & $0.5 \mathrm{~Hz}$ \\
\hline $\begin{array}{c}\text { High frequency bound of } \\
\text { the band-pass filter }\end{array}$ & $1.5 \mathrm{~Hz}$ & $1.5 \mathrm{~Hz}$ \\
\hline Magnification Factor & 75 & 75 \\
\hline Frame rate & $30 \mathrm{~Hz}$ & $60 \mathrm{~Hz}$ \\
\hline Attenuation factor & 0.1 & 0.1 \\
\hline
\end{tabular}

A region of interest was identified from which to extract a pulse measurement signal. Image segmentation is discussed in detail in Chapter 5; in this section the ROI was an area on the subject's chest. A spatial mean was calculated within the ROI and examined temporally. Each of these resulting signals was filtered with a fourth-order, zero-phase, Butterworth lowpass filter, were normalized between 0 and 1 , and examined.

The thermal video was then subjected to EVM, using the same parameters as for the light video: a wide passband and relatively low magnification factor. The enhanced thermal output video was examined. A thermal mean intensity signal, representative of mean temperature, was calculated spatially from within the same ROI and examined temporally. This signal was filtered using a fourth-order, zero-phase, Butterworth lowpass filter. The filtered mean intensity signals resulting from the same ROI in the light and thermal videos were compared to determine accuracy. 
The original thermal video was subjected again to EVM, this time using a narrow bandpass filter and a higher magnification factor. The narrow passband was targeted; it was chosen to be within the former wide passband, and to enclose the frequencies extracted from the subject's chest, using the wide passband. The narrow passband EVM enhanced output thermal video was examined as before; the chest ROI mean intensity signal was found, filtered and compared to the ECG signal to ensure accuracy. The ECG signal was low pass filtered, normalized to between 0 and 1 , and aligned with the light and thermal video data to validate findings.

The resulting light video mean intensity signal was strongly correlated with the ECG signal; both oscillating at $\mathrm{f}=0.9 \mathrm{~Hz}$ (approximately 54 beats per minute), and both depicted in Figure 6.4, where the normalized amplitudes are plotted at staggered baseline values so each signal can be seen clearly.

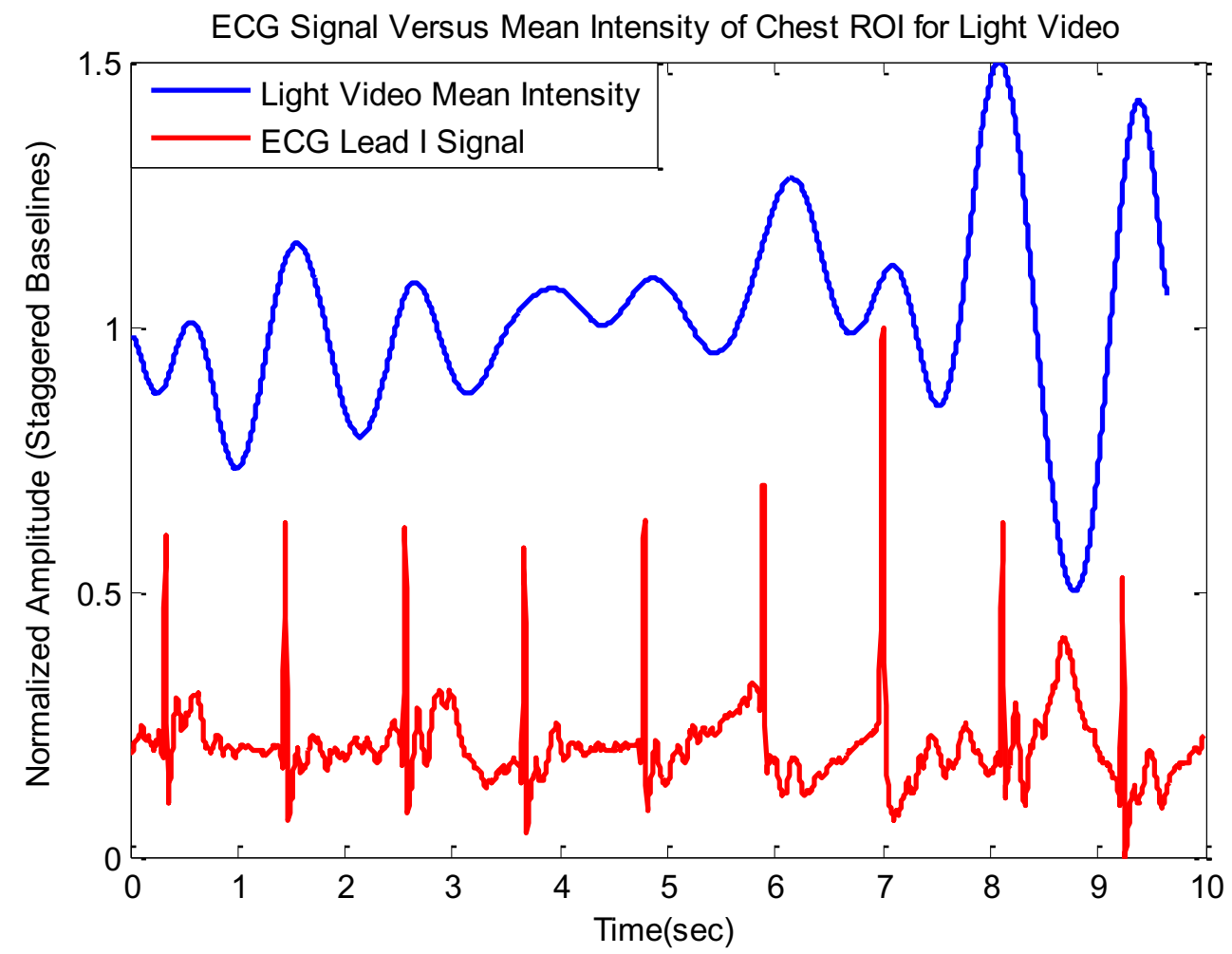

Figure 6.4 Light video ROI mean intensity signal (EVM bandpass filter 0.5 to $1.5 \mathrm{~Hz}$ ) versus ECG signal. 
The thermal video mean intensity signal was compared to the previously extracted and validated enhanced visible light video mean intensity signal. This comparison is depicted in Figure 6.5, where a staggered baseline has again been used to depict both normalized signals clearly. While there are some variations between signals, the correlation is visually clear. The frequencies of the extracted mean intensity signals were in the range of $\mathrm{f}_{\text {thermal }}=0.8 \mathrm{~Hz}$ (48 heart beats per minute) $f_{\text {light }}=0.9 \mathrm{~Hz}$ (54 heart beats per minute). The previous EVM filter was then adapted to enclose these frequencies tightly. The original thermal video was again subjected to EVM processing, this time using a narrow bandpass filter and a higher magnification factor. The EVM parameter values for this adapted filter can be seen in Table 6.2.

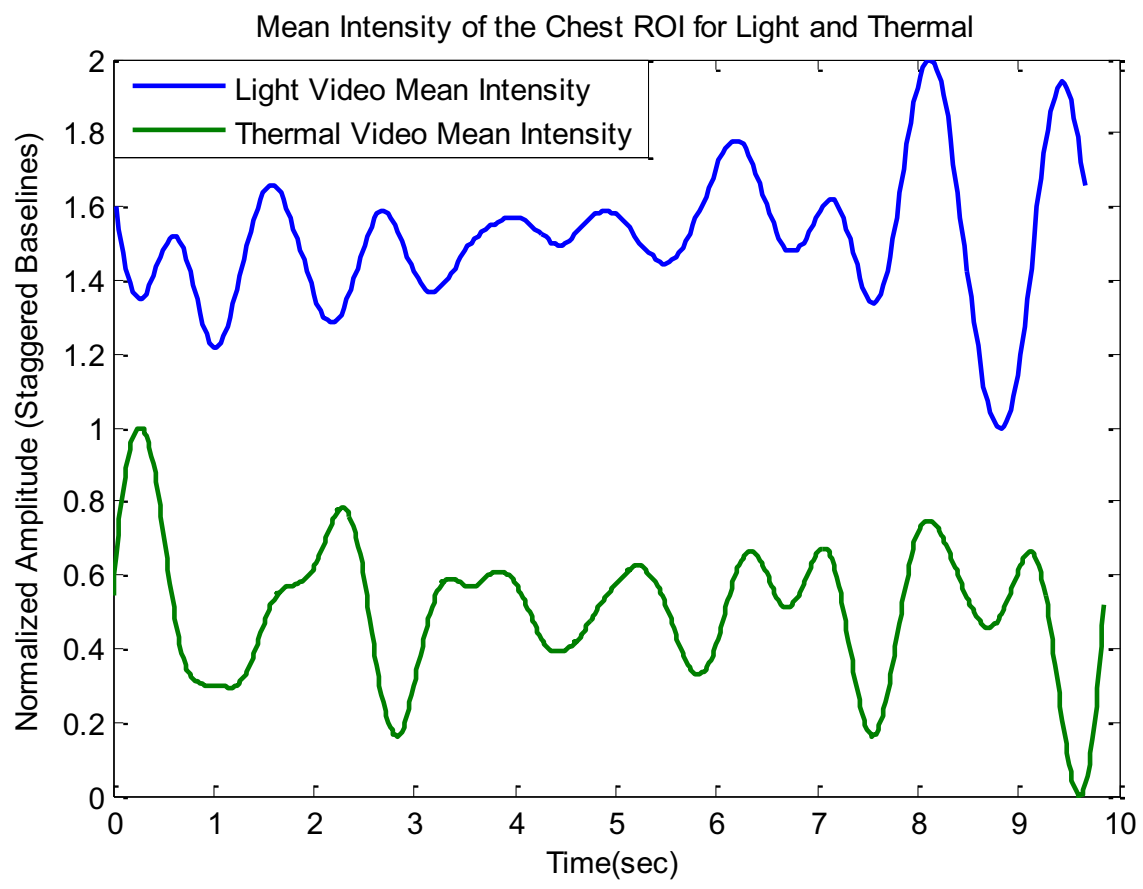

Figure 6.5 Light video ROI mean intensity signal versus thermal video ROI mean intensity signal (EVM bandpass filter 0.5 to $1.5 \mathrm{~Hz})$. 
Table 6.2 EVM Parameter Values for Narrow Passband

\begin{tabular}{|c|c|}
\hline Parameter & Thermal Video \\
\hline $\begin{array}{c}\text { Low frequency bound of } \\
\text { the band-pass filter }\end{array}$ & $0.75 \mathrm{~Hz}$ \\
\hline $\begin{array}{c}\text { High frequency bound of } \\
\text { the band-pass filter }\end{array}$ & $1.0 \mathrm{~Hz}$ \\
\hline Magnification Factor & 120 \\
\hline Frame rate & $60 \mathrm{~Hz}$ \\
\hline Attenuation factor & 0.1 \\
\hline
\end{tabular}

Following EVM processing, the ROI mean intensity signal was again calculated. This signal was compared with the ECG signal, both of which are normalized, and plotted at staggered baselines as depicted in Figure 6.6. It can be visually and quantitatively confirmed that the extracted thermal signal representing heart rate resulting from the EVM narrow band pass filter displays a strong agreement with the ECG signal; again both signals oscillate at $\mathrm{f}=0.9 \mathrm{~Hz}$ (approximately 54 beats per minute).

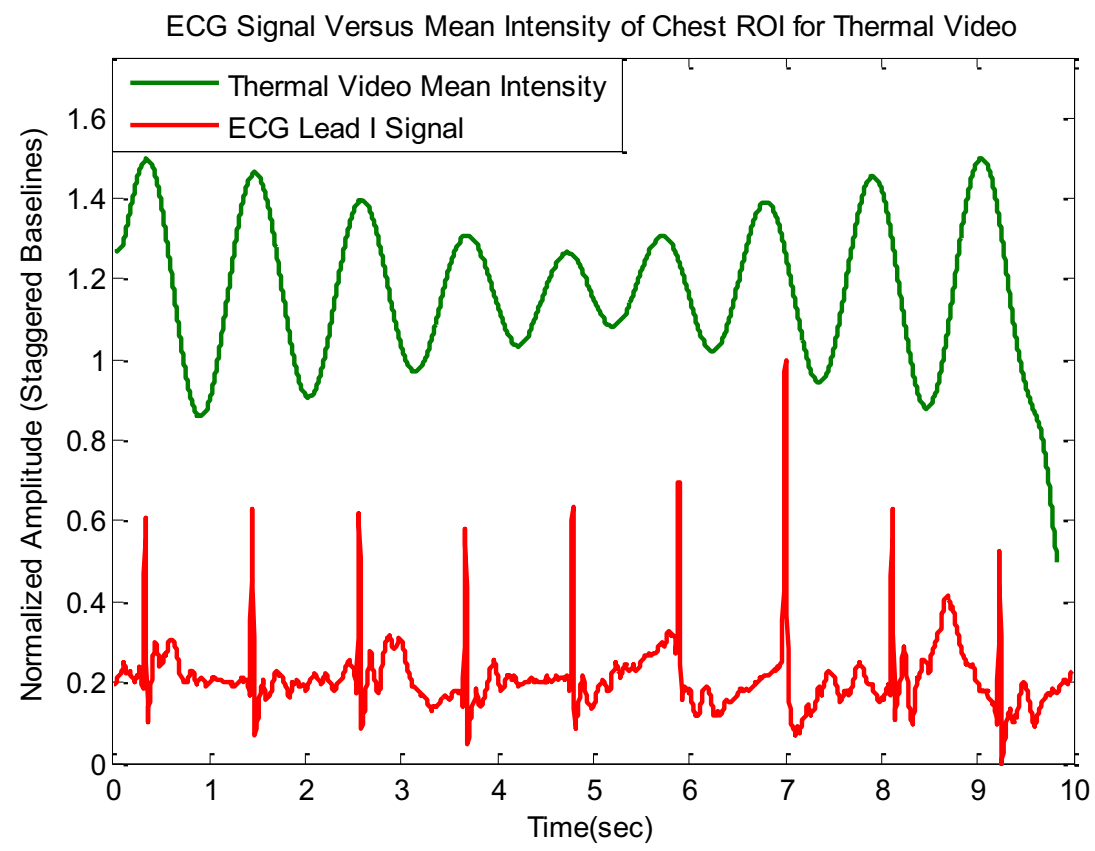

Figure 6.6 Thermal video ROI mean intensity signal (EVM bandpass filter 0.75 to $1 \mathrm{~Hz}$ ) versus ECG signal. 
These results suggest that, using AEVM processing and an anatomically viable region of interest, one may be able to identify biological signals instead of amplified noise. This theory was tested using thermal video of one subject; the mean intensity signal from the same anatomical region in thermal video was compared with the enhanced light video mean intensity signal. The correlation, while not perfect was still visually identifiable. This validated that the oscillations were not simply amplified noise; the EVM and post-processing repeated with the thermal video, using a narrow passband and higher amplification rate resulted in a mean intensity signal strongly correlated with the ECG signal.

The described analysis demonstrated that thermal video in conjunction with EVM and postprocessing can reveal biological signals. These results also indicate that when the biological signal frequency is unknown, an adaptive method can be applied wherein one employs EVM with a wide bandpass filter to identify the signal of interest, then re-processes, employing a targeted narrow bandpass filter to amplify the signal of interest, rather than simply amplifying noise.

\subsection{Video Processing Parameter Testing}

Application of video-temporal processing such as EVM (and AEVM) to thermal video data has only been examined more recently [68],[19],[18]. As a recent body of work, and with several parameters affecting system outputs, the developed algorithms require robust testing to identify specific patterns and behaviors, specifically in non-ideal conditions. This chapter achieves this by first generating over 500 simulations of human pulse thermal video, then corrupting the videos with prescribed, increasing amounts of noise and inputting these videos to the algorithmic system. System parameters were varied, and the effect of noise was observed. Understanding and 
characterizing patterns of failure is important in identifying when the algorithm outputs may become inaccurate.

\subsubsection{Video Generation}

In other parts of the thesis, thermal video was captured of subjects and then examined. This section tests video magnification algorithms by simulating thermal video of human subjects, altering variables in a known manner, subjecting these videos to video processing algorithms and examining results. Thermal video of a subject was examined to validate simulation results. An approximate 2.5 minutes of thermal video data were captured of the subject sitting quietly. Details surrounding data capture procedures can be found in Chapter 3.

Video data can be represented in several different ways, namely in a 3 channel Red-GreenBlue (RGB) format or a 1 channel intensity format. Thermal video data can be presented in color but is considered in this thesis to be intensity data. A simplified representation of a subject was first created. This included a large circle for the head area, and a semi-circle representing the shoulders. Each pixel within the area representing the face was given an intensity value of 0.5 and from this baseline value, a sinusoidal pattern was imposed over time. A generalized example of this is depicted in Figure 6.7. These intensity changes are intended to represent blood flow to the face, which has been found to present in a sinusoidal manner [18]. 


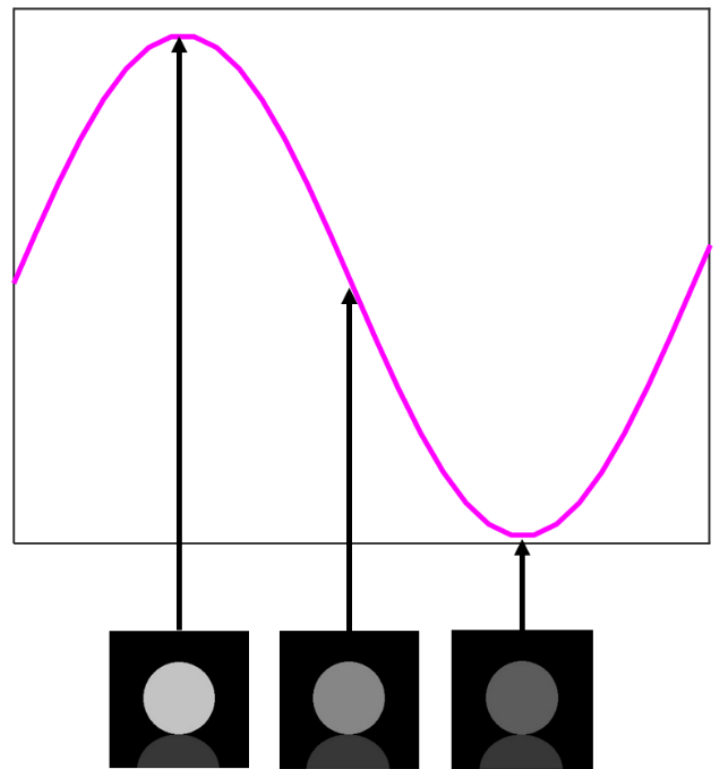

Figure 6.7 Depiction of the area of interest's sinusoidal changes.

Videos were generated to represent a wide range of heart rates; from 20 beats per minute (bpm), to $160 \mathrm{bpm}$ in $5 \mathrm{bpm}$ increments, for a total of 29 heart rates. Frequencies were chosen to include heart rates outside of the range of normal, healthy adult heart rates, in order to test the system's response when the signal is outside of the target range. For each video, Gaussian noise was then added, with 18 different noise levels and 18 respective noise variances. Signal to Noise Ratio (SNR) is a measure that can be calculated many ways. This thesis considers a measure that is not necessarily a signal to noise ratio, but that quantifies the amount of noise present when compared to the signal. This measure was the Mean Square Ratio (MSR), which is defined in Equation 6.1 - Equation 6.3. 
Equation 6.1

Equation 6.2

$$
\left.M S_{\text {noise }}=\frac{1}{N} \sum_{x=1}^{X} \sum_{y=1}^{Y}\left(R O I_{x, y}\right)\right)^{2}
$$

$$
M S R=\frac{M S_{\text {signal }}}{M S_{\text {noise }}}
$$

Where $H R_{t}$ represents the value of the imposed heart rate signal at a given time, $t$, and $T$ represents the number of time instances.

Where $R O I_{x, y}$ represents the value of an image pixel within the ROI at a location, $(x, y)$, and $(X, Y)$ indicates the maximum size of the ROI.

Where $M S_{\text {signal }}$ and $M S_{\text {noise }}$ are calculated as in the equations above.

Equation 6.1 indicates the calculations used to obtain the mean square signal value. All signals have the same amplitude and sampling frequency so this value remains the same for all the generated signals.

Equation 6.2 indicates the calculation used to obtain the mean square noise value. There is one mean square noise value for each noise level. Equation 6.3 indicates the simple ratio calculation required to obtain the MSR values. Each nominal noise level was created using specific noise variances, the respective MSR values for which can be seen in

Table 6.3. Video generation resulted in 522 simulated thermal videos of subjects with varying, controlled heart rates contaminated with varying, controlled amounts of Gaussian noise. 
Table 6.3 Noise variance and mean square ratio

\begin{tabular}{|c|c|c|}
\hline Noise Level & Noise Variance & MSR \\
\hline 1 & 0 & $\infty$ (no noise) \\
\hline 2 & 0.01 & 49.998 \\
\hline 3 & 0.1 & 5.018 \\
\hline 4 & 0.5 & 1.333 \\
\hline 5 & 1 & 0.974 \\
\hline 6 & 5 & 0.654 \\
\hline 7 & 10 & 0.600 \\
\hline 8 & 50 & 0.541 \\
\hline 9 & 100 & 0.528 \\
\hline 10 & 200 & 0.520 \\
\hline 11 & 300 & 0.517 \\
\hline 12 & 400 & 0.515 \\
\hline 13 & 500 & 0.513 \\
\hline 14 & 1000 & 0.511 \\
\hline 15 & 5000 & 0.508 \\
\hline 16 & 10000 & 0.504 \\
\hline 17 & 50000 & 0.505 \\
\hline 18 & 100000 & 0.502 \\
\hline
\end{tabular}




\subsubsection{Video Data Analysis}

Data segmentation was first performed as described in Chapter 5. For this application an area representing a subject's forehead was first identified, as depicted in Figure 6.8. The same area was identified on the actual subject, as can be seen in Figure 6.9.

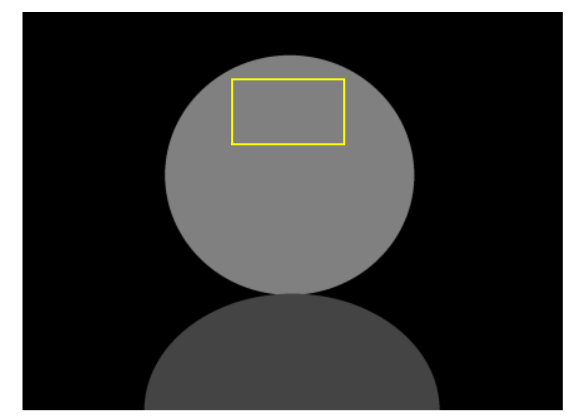

Figure 6.8 Region of interest: the simulated subject's forehead

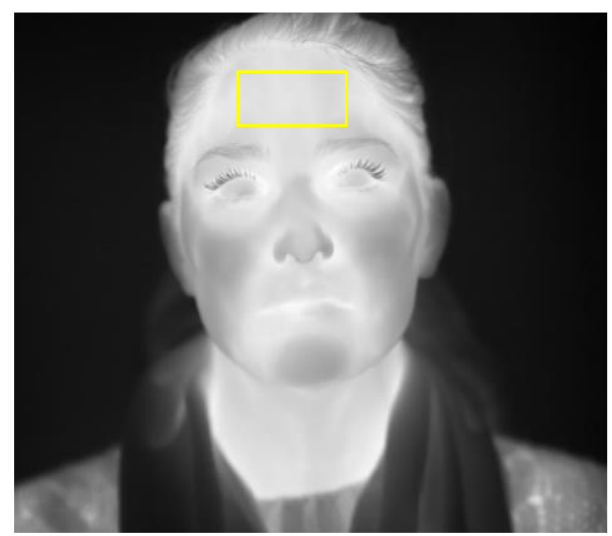

Figure 6.9 Region of interest: the subject's forehead

Each of the 522 videos generated were then subjected to spatio-temporal video processing. The video processing techniques examined include both EVM and AEVM, described in Sections 6.2 and 6.3, respectively.

Both EVM and AEVM algorithms were systematically tested using signals extracted from the 522 generated videos. These algorithms require several user-determined parameter values. 
These parameters include the cut-off frequencies of the temporal filter, the magnification factor, the frame rate, and the attenuation factor. The cut-off frequencies were varied for testing. The other parameters remained constant, the values for which are detailed in Table 6.4.

Table 6.4 EVM Parameter Values

\begin{tabular}{|c|c|}
\hline Parameter & Thermal Video \\
\hline Magnification Factor & 1 \\
\hline Frame rate & $30 \mathrm{~Hz}$ \\
\hline Attenuation factor & 0.1 \\
\hline
\end{tabular}

Several frequency ranges were chosen in decreasing bandwidth; 30-150 bpm, 45-130 bpm, 60-120 bpm, 72-108 bpm, 84-96 bpm and 87-93 bpm. For each of 522 simulated thermal videos, EVM processing was applied once for each bandwidth; a total of six processing loops for each video, resulting in 3132 extracted signals. For each signal, a fast fourier transform was used to transform the time signals to the frequency domain. The largest peak within the frequency range of interest was chosen as the detected heart rate. The error between the detected heart rate and the known heart rate was calculated in absolutes, the results for which are described in the following section.

The same processing was done for the AEVM algorithm, where there were only two bandwidths; the initial, wider bandwidth of 30-150 bpm, and then a narrower bandwidth defined as $10 \mathrm{bpm}$ above and below the signal frequency detected in the first iteration. The error between the detected heart rate and the known heart rate were calculated in absolutes, the results for which are described in the following section. 
Also examined were the results from processing thermal video of an actual subject. The video processing was the same as was applied to the simulated videos; the results for which were used for validation purposes and are described in the following section.

\subsubsection{Parameter Testing Results}

Spatio-temporal processing of subject data was performed for validation purposes, the results for which are presented in Figure 6.10. The black line indicates the original signal extracted from within the ROI identified in Figure 6.9. The other two plot lines are the results of video processing with a wide and narrow filter width (30-150 bpm and 87-93 bpm, respectively). In Figure 6.10, the wide filter results are displayed in magenta, and the narrow filter results displayed in cyan. The wide filter video processing determined the heart rate (over the 2.5 minutes of data) to be $64 \mathrm{bpm}$, and the narrow filter video processing determined the heart rate to be 90.5 . The actual heart rate was approximately $66 \mathrm{bpm}$. The widest filter was able to pick up the signal frequency (though not entirely accurate), where the narrowest filter was not; it was simply outputting amplified noise. These results are as expected and are consistent with previous results, as well as those from simulated thermal videos. 


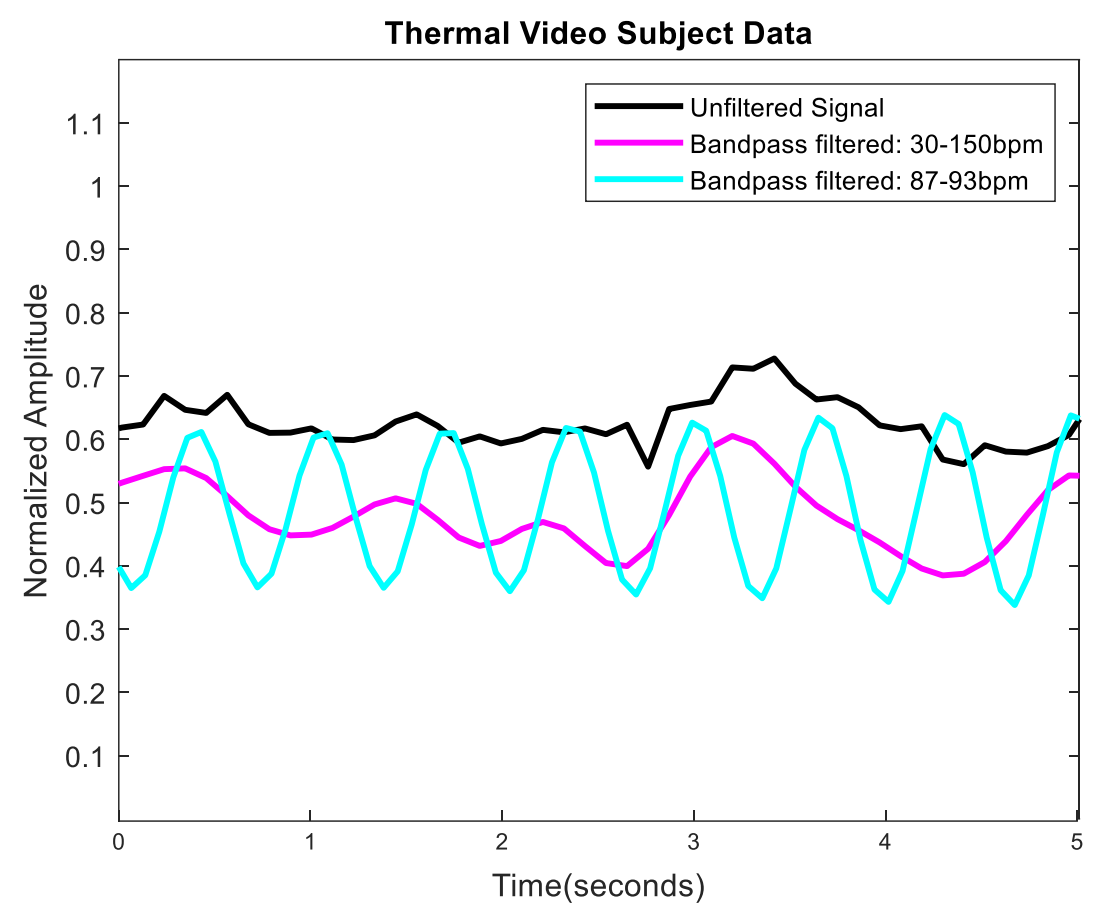

Figure 6.10 Original and filtered heart rate signals extracted from a subject, processed with two separate bandpass filters

The spatio-temporal video processing resulted in output signals that accurately portrayed the imposed heart rate and displayed noise patterns proportional to those imposed. The processing also resulted in filtered output signals consistent with patterns expected from respective bandwidths. An example of these results can be seen in Figure 6.11. These signals correspond to 5 seconds of video data generated at a frequency of $1 \mathrm{~Hz}(60 \mathrm{bpm})$ with Gaussian noise added to the image at a noise variance of 0.1 . The extracted, unfiltered signal is plotted in blue, where the signal frequency is clear, and a small amount of noise is present and visible. The signal plotted in red is the result of filtering with a bandpass of $30-150 \mathrm{bpm}$. This is a relatively wide bandpass, with the original signal frequency of $60 \mathrm{bpm}$ clearly within these boundaries. This signal is therefore a cleaner version of the original signal. The signal plotted in orange is the result of bandpass filtering between $87-93 \mathrm{bpm}$. This bandpass does not include 
the heart rate of $60 \mathrm{bpm}$ and the results reflect this; the signal frequency is seen to be closer to $90 \mathrm{bpm}$; the center frequency of the narrower bandpass filter. These results were consistent among signals; demonstrated by cumulative results drawn from the 3132 extracted signals discussed in the following.

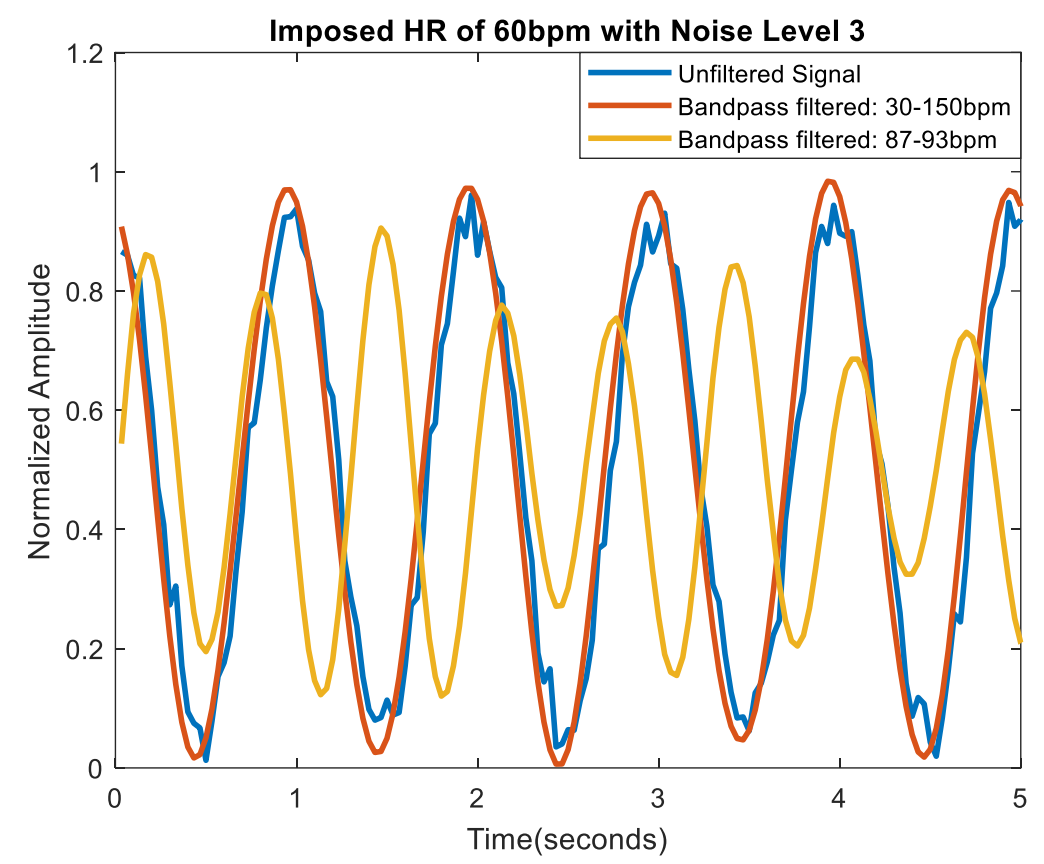

Figure 6.11 Original and filtered heart rate signals at a frequency of $60 \mathrm{bpm}, \mathrm{MSR}$ of 5 , subjected to two separate bandpass filters

Cumulative results were collected by examining heart rate error; each signal resulting from the iterative application of EVM to each noise altered video was reduced to one number by identifying the estimated heart rate (fast fourier transform, followed by peak detection) and calculating the difference between the estimated and known heart rates. This heart rate error data was plotted for each noise level, each signal and each applied bandwidth.

The plot in Figure 6.12 depicts heart rate errors for signals extracted from videos with no added noise. For reference, a perfect result with no estimate error would be a horizontal line at 
a value of zero. The plotted results are as hypothesized; if the imposed heart rate lies within the filter cut-off frequencies, then the signal is detected accurately. The estimates become inaccurate when the filter boundaries do not contain the actual signal frequency. Because the bandpass filters are not ideal in reality, the filter boundaries are approximately $5 \mathrm{bpm}$ outside of where the error drops to zero. In addition, the narrower the filter, the more likely the algorithm will tend towards the center frequency. For this testing, the filters are prescribed; the center frequency is always $1.5 \mathrm{~Hz}(90 \mathrm{bpm})$. This is evident in the plots, as the signal representing the heart rate error for the narrowest filter depicts a result of $90 \mathrm{bpm}$, regardless of the actual heart rate. An example signal is depicted in Figure 6.13. This figure depicts an original ROI mean signal at a prescribed heart rate of 90 , and no added noise, to demonstrate what signals at this noise level appear like.

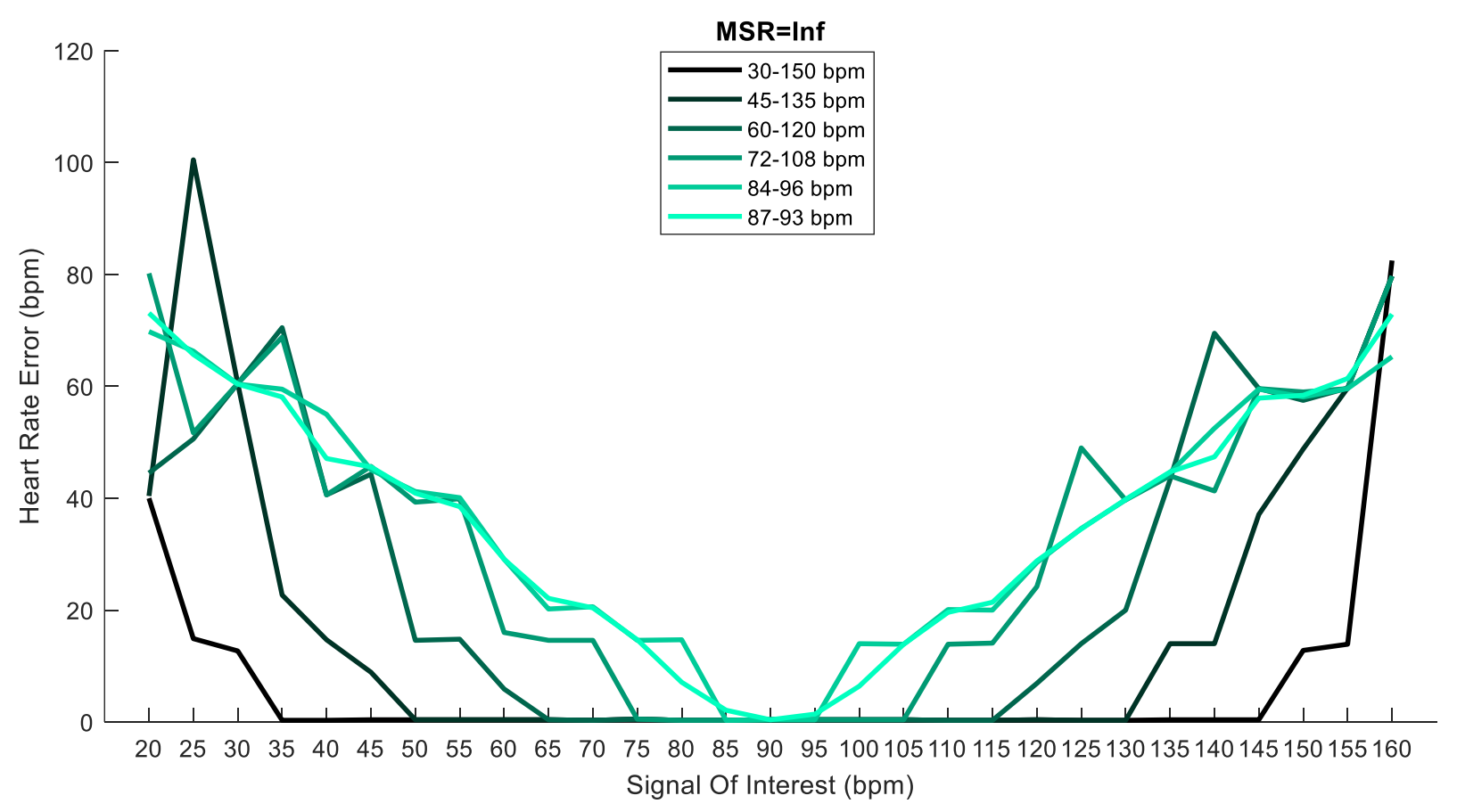

Figure 6.12 Cumulative results, noise level 1 


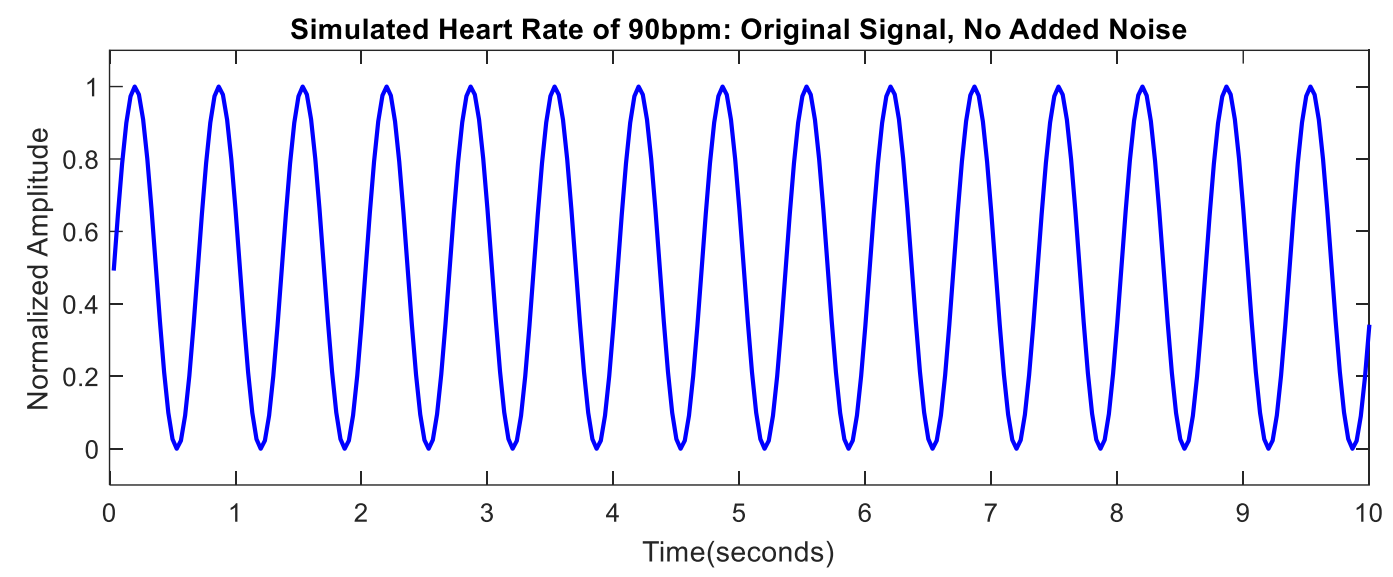

Figure 6.13 Mean intensity signal, pre-filtering for noise level level 1

The following four plots, Figure 6.14 - Figure 6.17 depict the same results but for heart rate estimate errors calculated from videos subjected to Gaussian noise with noise variances of 100 and 500 and MSRs of 0.53 and 0.51, respectively. Noise level 9, depicted in Figure 6.14, appears to be the noise level at which inaccuracies begin to appear. Figure 6.15 depicts an original ROI mean signal at a prescribed heart rate of 90 , and noise level 9, demonstrating what signals at this noise level appear like. It is clear that there is a significant increase in noise, and the prescribed heart rate is not visible to the naked eye. Figure 6.16 depicts cumulative results at noise level 13; the heart rate estimates become quite inaccurate and start to approach the center frequency of $1.5 \mathrm{~Hz}(90 \mathrm{bpm})$. Figure 6.17 displays an original ROI mean signal at a prescribed heart rate of 90 , and noise level 13, depicting what signals at this noise level appear like. There is not an obvious difference visually between Figure 6.15 and Figure 6.17; both indicate the noise levels are high enough that the prescribed heart rate is no longer visible to the eye. While the signals cannot be distinguished visually, the EVM algorithm is able to distinguish between the noise levels, and in one case extract the underlying heart rate. 


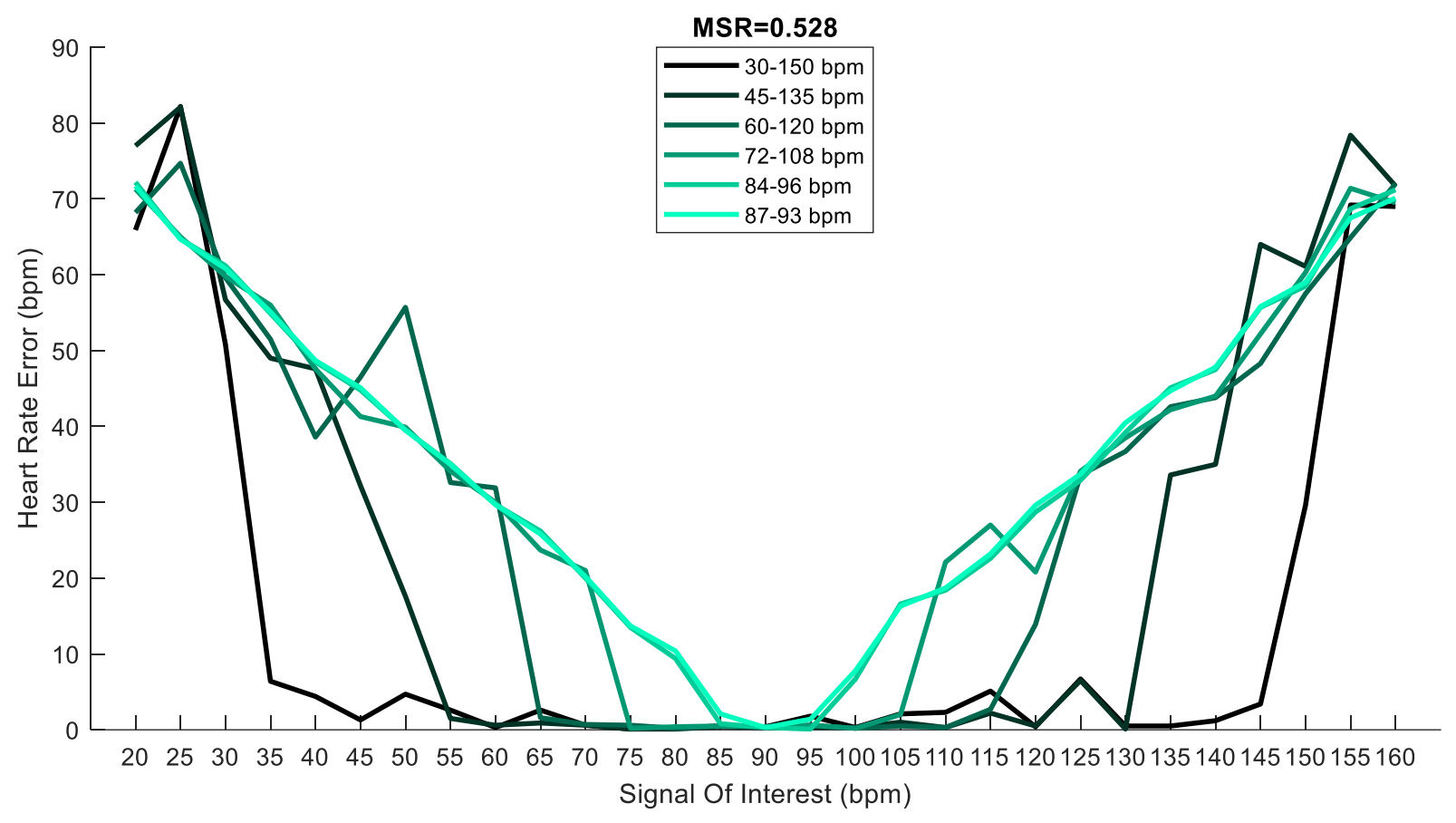

Figure 6.14 Cumulative results, noise level 9

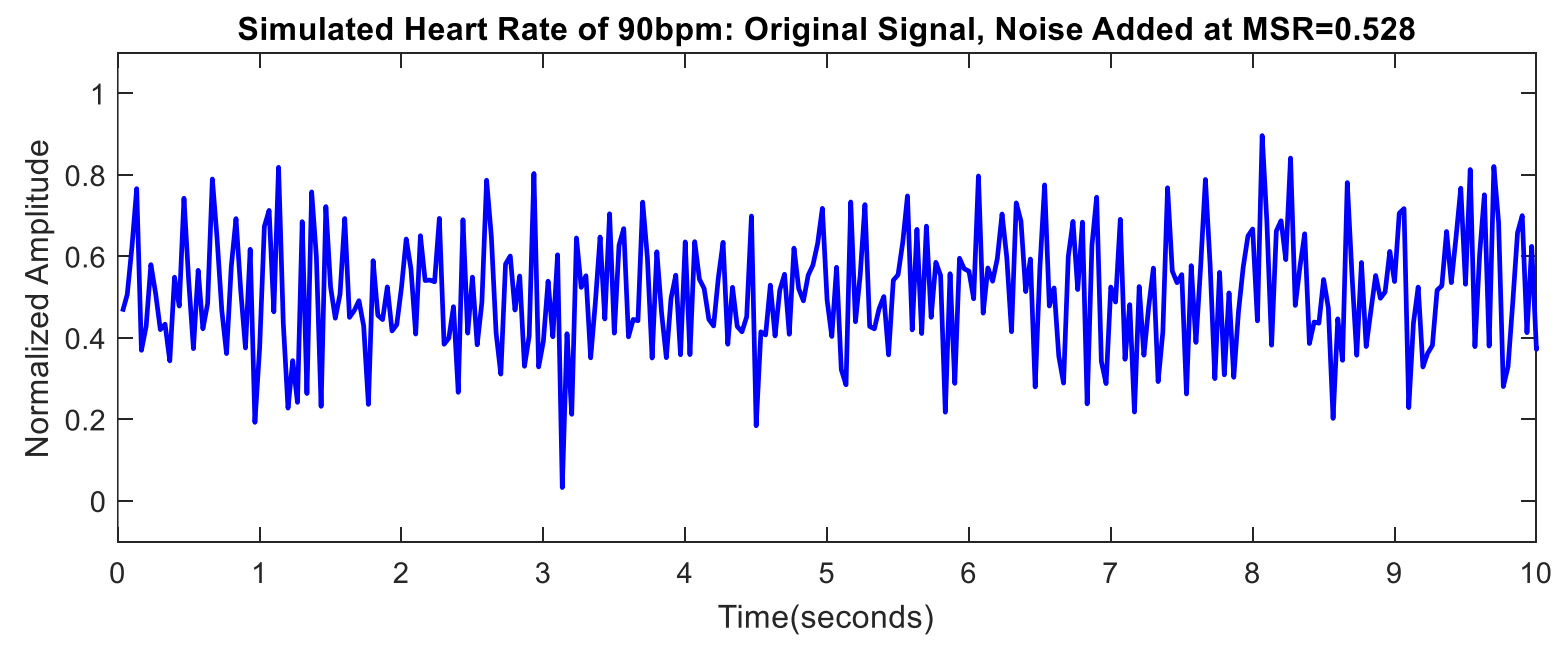

Figure 6.15 Mean intensity signal, pre-filtering for noise level 9 


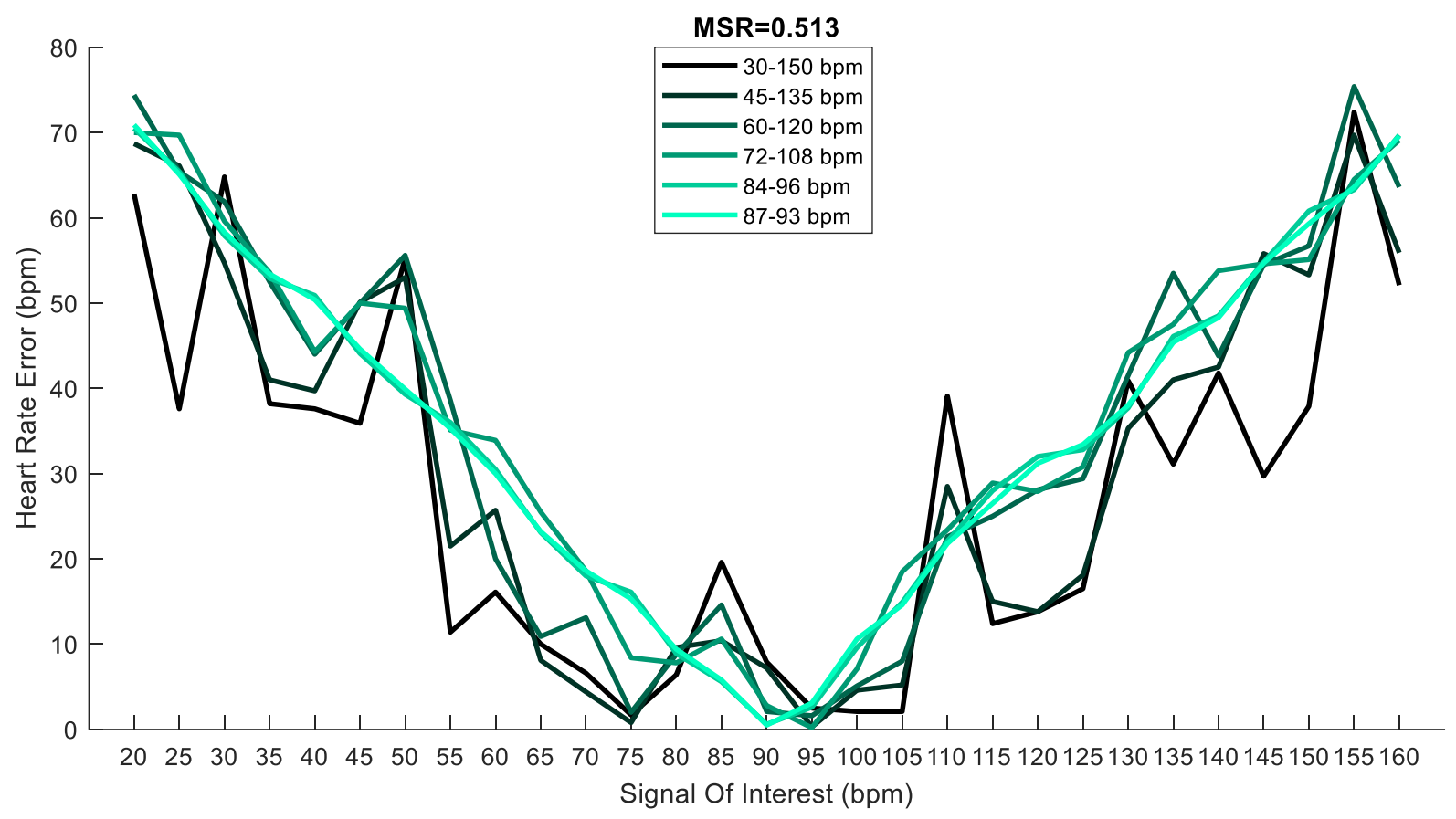

Figure 6.16 Cumulative results, noise level 13

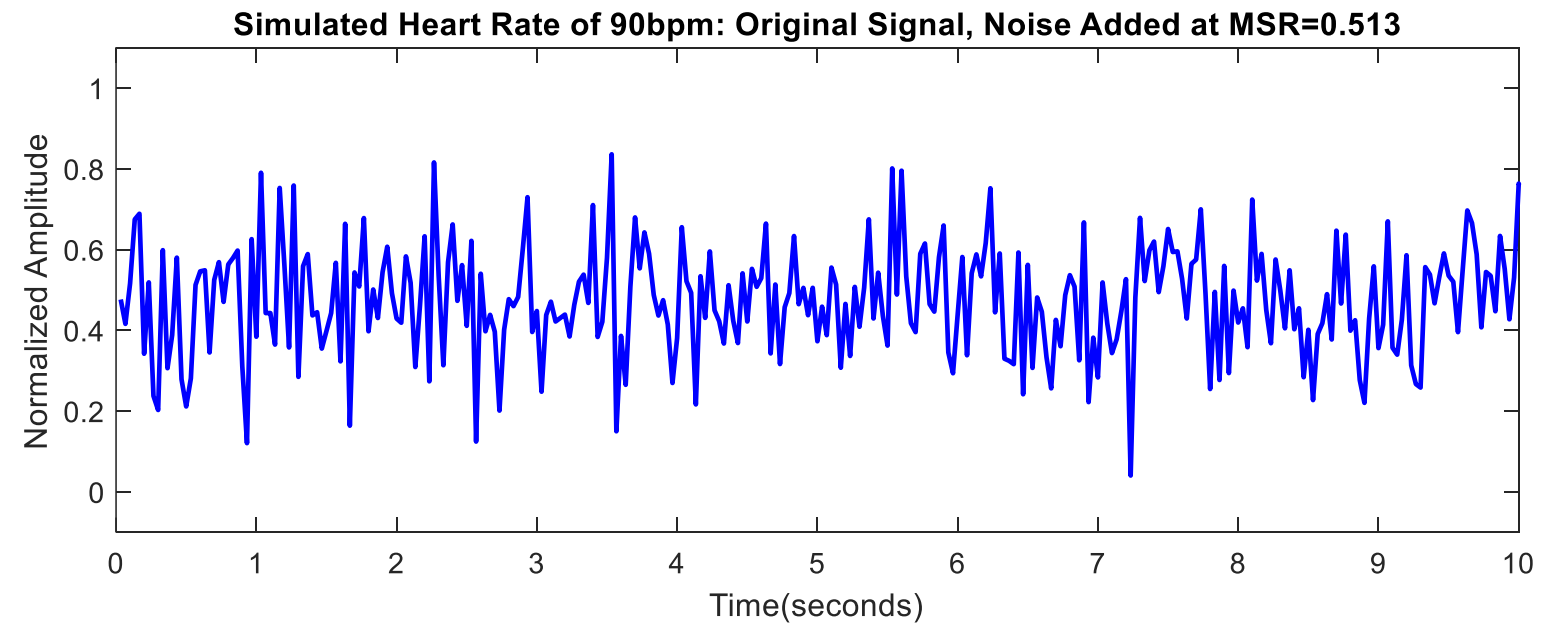

Figure 6.17 Mean intensity signal, pre-filtering for noise level 13

Figure 6.18 depicts the MSR value for each noise level. It can be observed that the MSR value approaches a limit of 0.5 . This limit exists because the simulated thermal video was greyscale and there is therefore a limit to the amount of noise that can be added before one 
would encounter a ceiling and floor effect. The heart rate estimate error begins to increase after an approximate MSR value of 0.55 , which occurs around Noise Level 8 . These results are consistent with the heart rate estimate error results.

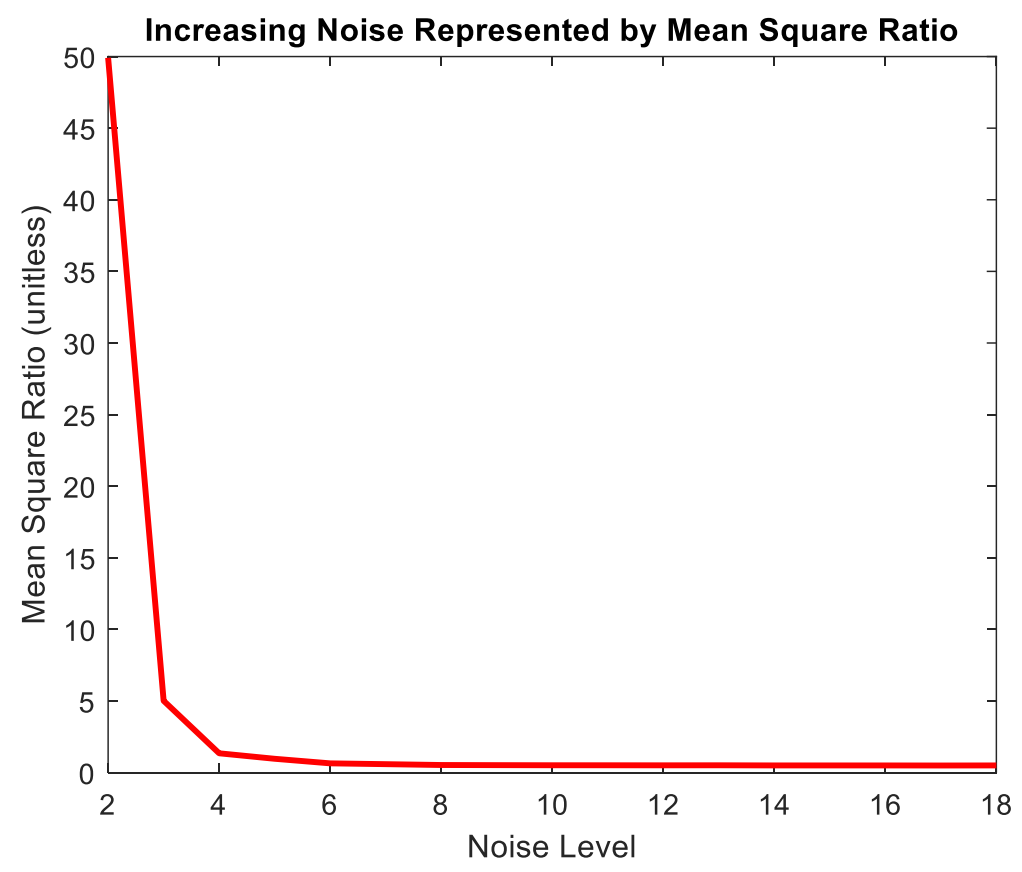

Figure 6.18 Noise levels and respective MSR values

The videos generated are unrealistic in that they represent ideal conditions. This includes no subject movement, no variety in face shape, color or behaviours, etc. These simulated videos with ideal conditions are advantageous when testing system parameters and limits as it allows for the isolation of specific results. It was disadvantageous in testing the AEVM, as the AEVM performs much better when there are more confounding factors and the underlying signal is not as computationally clear. This is demonstrated in the vital signal results chapters (Chapters 7 to 9), where the results of applying the AEVM to both human subjects and a mechanical simulation system are presented and discussed. As depicted in Figure 6.12 and Figure 6.14, the signal was identifiable using the EVM and the widest filter bandwidth until a noise level of 9 
$(\mathrm{MSR}=0.53)$. This is point at which the AEVM was able to improve the results using two iterations of EVM; one with a wider bandwidth to identify an underlying signal, and the second using a narrower bandwidth, here defined as $10 \mathrm{bpm}$ above and below the heart rate detected in the first EVM iteration. This is visualized in Figure 6.19 and Figure 6.20, where Figure 6.20 depicts the same data, but with an adjusted y axis so as to display more detail. It can be observed that the AEVM improved estimates by as much as $7 \mathrm{bpm}$, in ideal conditions. It can be seen throughout this thesis that this algorithm also performs well on real subjects and unideal conditions.

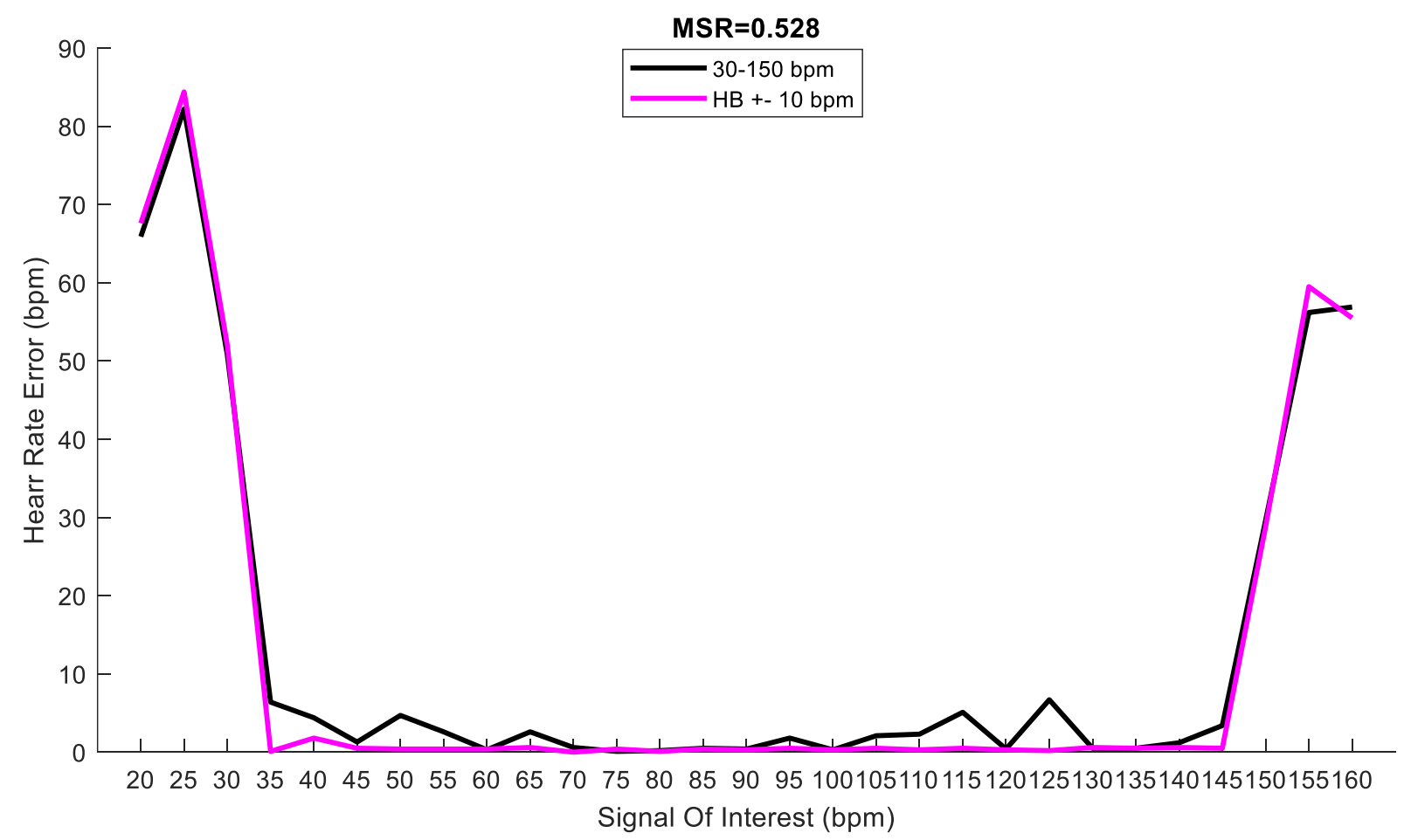

Figure 6.19 AEVM applied to videos subjected to noise level 9 


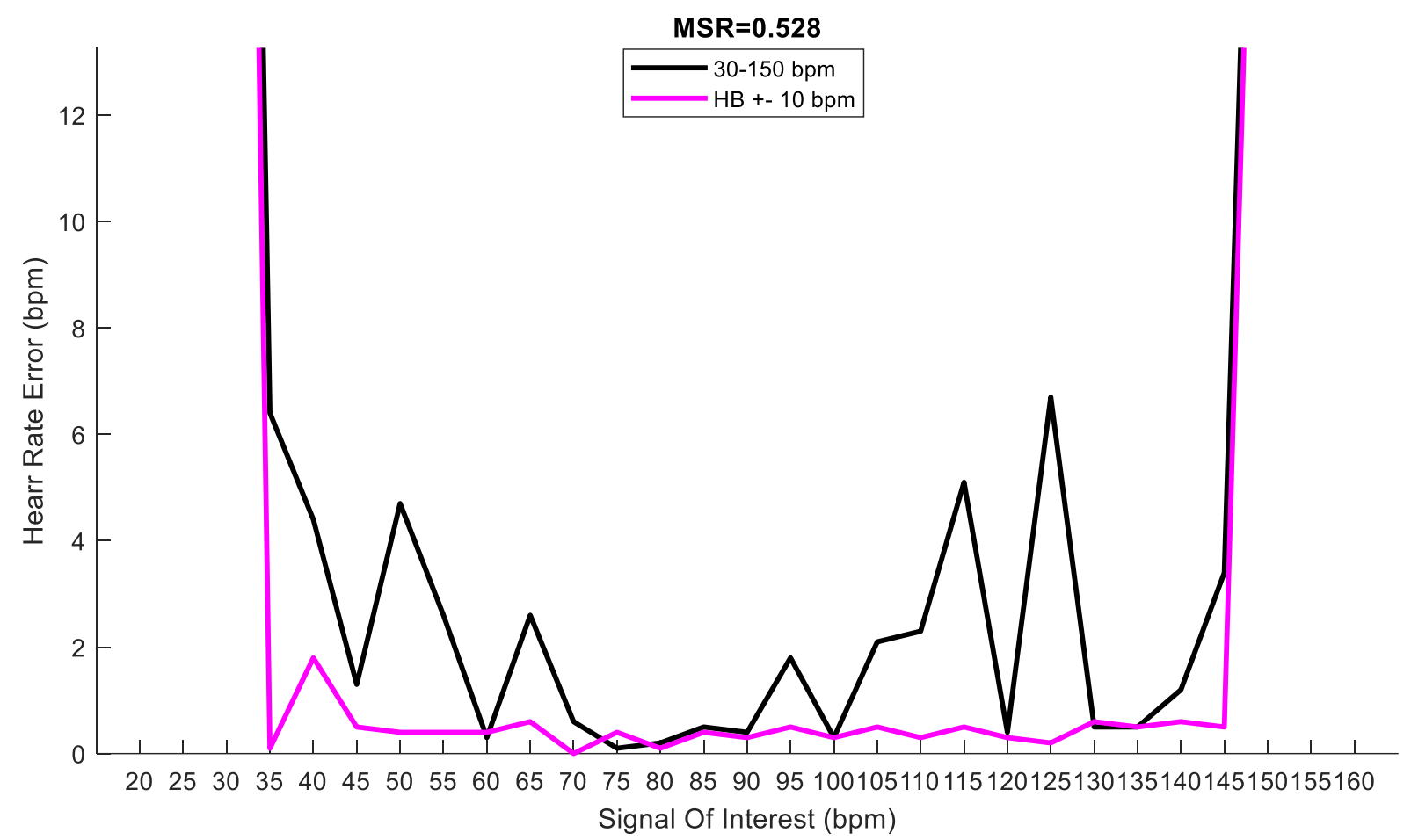

Figure 6.20 AEVM applied to videos subjected to noise level 9 with adjusted y axis

\subsubsection{Parameter Testing Discussion}

This section aimed to test the spatio-temporal algorithms used to extract biosignals from thermal video of subjects. Specifically, to test the accuracy of the system outputs in response to noise. Simulated thermal video data was generated of a subject with facial perfusion at a prescribed frequency. For each of 29 videos at prescribed signal frequencies, a new video was created with gaussian noise added at one of 18 different noise variances. This resulted in 522 simulated videos. These videos were passed as inputs to the algorithmic system. The processing was performed 6 times for each video, each iteration with a different filter width. This resulted in 3132 signals, which were examined for accuracy and overarching patterns.

The algorithm results were first compared with results of actual thermal video data having been subjected to spatio-temporal video processing. The patterns of behavior in the signals 
resulting from the subject videos were consistent in what has been observed in previous studies, as well as what has been observed in extracted signals from simulated thermal video. This result suggests that the simulated thermal video presents similarly enough to real thermal video data, that conclusions can be drawn about spatio-temporal video processing of thermal video, through the testing of spatio-temporal processing using hundreds of simulated thermal videos. Simulated thermal videos are advantageous in testing as all variables are controlled, where real thermal video data is subject to unpredictable variable changes.

In examining the signals resulting from spatio-temporal processing of simulated thermal video, the results were consistent with what was expected. For a prescribed video representing heart rate, the signal resulting directly from the video was oscillating at the given frequency and was displaying a proportional amount of noise (to what was imposed). Following spatiotemporal processing at 6 different filter widths, the patterns again were as expected. If the actual signal frequency was within the filter passband, the signal was detected at an accurate heart rate. If the actual signal frequency was outside of the filter passband, a signal was detected at an inaccurate frequency; the result of amplified noise. The filter width played a role in the signal results as well; a wider passband resulted in a noisier signal with a weaker amplitude (not evident in normalized plots) and a narrower passband resulted in a cleaner signal with stronger amplitude. These results are consistent with previous findings and emphasize the ease with which a user could produce a clean, but inaccurate biosignal using a narrow passband and high amplification factor [9],[68].

For the cumulative results, some results were expected, and some results were unforeseen. The same patterns were observed individually as when considering thousands of signals. If the original signal was inside the filter passband, the processing resulted in an accurate heart rate 
estimate. If the signal was outside of the filter passband, the processing would amplify noise, resulting in an inaccurate heart rate estimate. What was unknown was the effects of increasing noise on the system outputs; how and when the noise would force the system to failure. The system produces accurate results for noise levels ranging from no noise to an MSR of 0.53. At this point it is clear the added noise is affecting the heart rate estimate accuracy. This result worsens with increasing noise until an MSR of 0.51 , at which point the system can no longer distinguish between noise and an underlying signal. Knowing at what point the system fails allows for informed use of the algorithms; understanding at what point the result is simply amplified noise.

One interesting cumulative result was that after the point at which noise started to affect the results, the heart rate estimates started to tend towards the center frequency of $90 \mathrm{bpm}$. This may be another indicator of false signal estimate; if a signal is simply presenting at the center frequency, the results may require further investigation.

The simulation data were also processed using the AEVM algorithms, and cumulative results were examined. The AEVM algorithm did not improve the results until the EVM algorithm began to fall subject to noise, around an MSR of 0.53 . This makes sense, as until that point, the EVM did not need an adaptive algorithm to improve already accurate results. When the EVM heart rate estimates became inaccurate, the AEVM was able to improve the heart rate estimates by as much as $7 \mathrm{bpm}$. The AEVM algorithm failed at the same point as the EVM algorithm; the adaptive method was unable to improve heart rate estimates at the failure point. The adaptive method is therefore most useful when there is enough noise present to affect the heart rate estimates, but not enough to cause system failure. These results demonstrated the effectiveness of AEVM, particularly in unideal conditions. 


\subsection{Conclusion}

This work demonstrated the potential of the proposed AEVM method to extract an existing physiological signal and not simply an amplified noise signal. Furthermore, this work demonstrated the potential for thermal video in conjunction with the proposed adaptive EVM methods to be used in the extraction of physiological signals.

This section tested the robustness of both EVM and AEVM algorithms by generating simulated thermal videos, adding varying amounts of imposed noise to these videos and using both EVM and AEVM to extract signals and estimated heart rates. The results suggest there are several patterns associated with system failure. Identification of these patterns is important in informing future use of these algorithms; understanding and recognizing symptoms of noise, filter width or amplitude error.

The developed adaptive method is employed in the following chapters of this thesis, to extract vital signals from thermal infrared and visible light video data from subjects. While this chapter focused on the use of EVM and AEVM to extract heart rate from thermal video (simulated and of real subjects), the results and conclusions of this chapter are applicable to the following chapters, which detail the application of AEVM and EVM to both light and thermal video of subjects to extract respiration rate, temperature and mobility measures in addition to pulse rate. 


\section{Chapter 7}

\section{Pulse Measurement Extraction}

This chapter builds upon the previous chapters by detailing the specific experimentation, data analysis, results and discussion associated with pulse.

\subsection{Introduction}

The entire algorithmic system is depicted in Figure 7.1 below, where this chapter focuses on the algorithmic processes and outcomes of pulse measurements, the component highlighted yellow. The following sections will detail specific image and signal processing methods used to estimate pulse measurements close to the heart (proximal measurements), pulse measurements at the extremities (distal measurements), and the effectiveness of the proposed methods in the presence of noise and signal abnormalities.

This chapter refers to several image processing methods detailed in previous chapters. This includes image smoothing and enhancement, detailed in Chapter 4, Region of Interest identification, detailed in Chapter 5, and Adaptive Eulerian Video Magnification (AEVM), detailed in Chapter 6. 


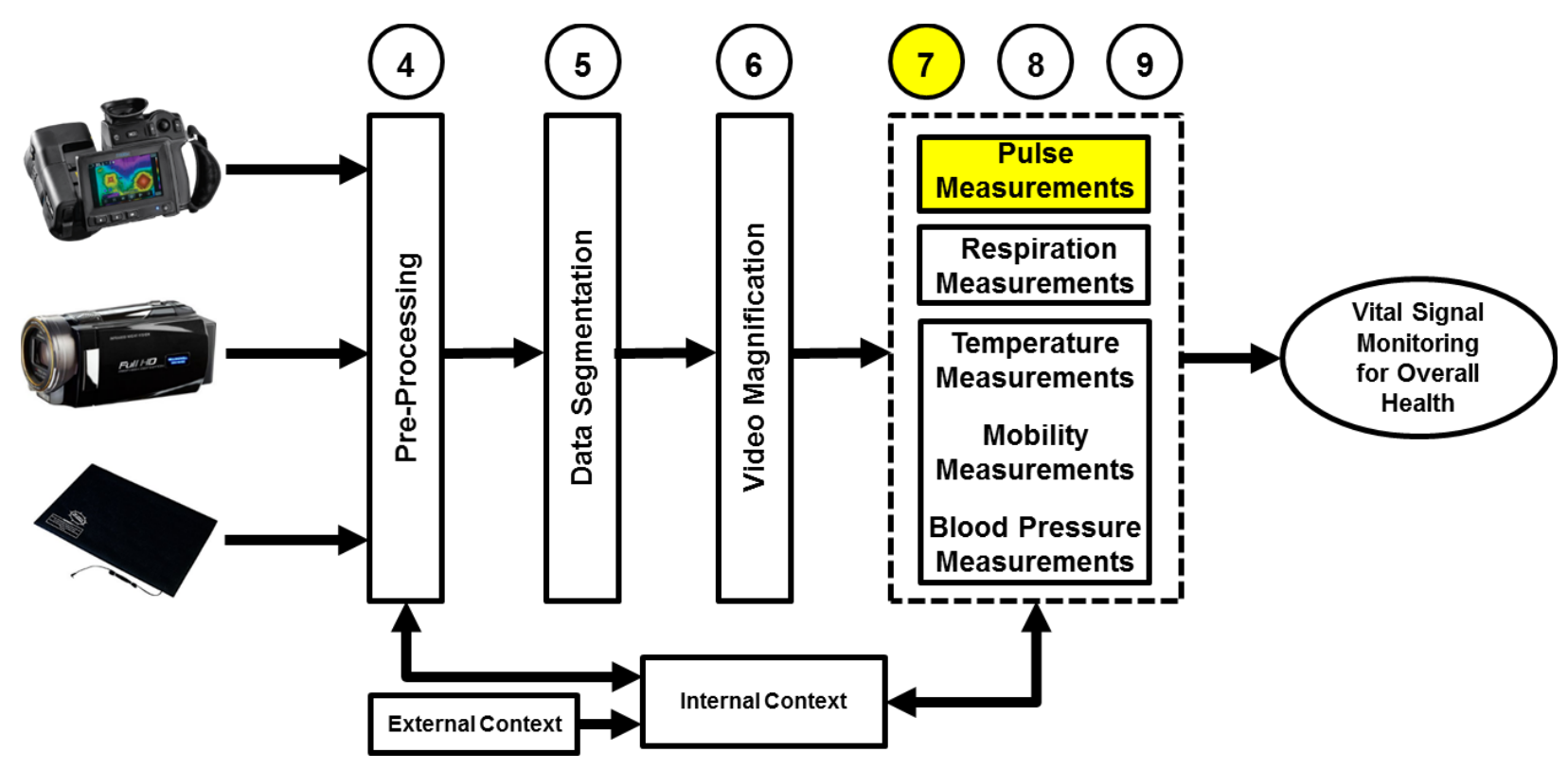

Figure 7.1 Algorithmic system with highlighted chapter focus

\subsection{Equipment and Experimentation}

The equipment used in pulse rate experimentation were inclusive of two thermal cameras, an HD digital camera, a biosignal chest strap measuring physiological signals, and a laptop. Technical specifications for all equipment are detailed in Chapter 4.

The TI (thermal infrared) cameras were used to gather thermal data from 16 subjects. For each TI video captured there was a concurrent VL (visible light) video of the subject gathered using an HD digital camera. Throughout experimentation, TI and VL video data were collected of different areas of a subject's body. For pulse rate, experimentation included data collection from subject's faces, upper bodies and the extremities (arms, hands, and feet). All data was collected and analyzed on the laptop.

The biosignal chest strap captures heart rate and breathing rate, among several other physiological signals. The device is worn by the user for the duration of data collection and 
removed afterwards for data download. The biosignal chest strap was used as the gold standard in data analysis, providing the true measurement of pulse rate in data analysis.

\subsection{Pulse Measurement Data Analysis}

This section details the specific algorithms developed in this thesis to extract pulse measurements from two different measurement sources, the Thermal Infrared (TI) camera and the Visible Light (VL) Camera. Pulse measurements in this chapter include the rate at which the heart beats, in addition to the shape, rhythm and strength of the pulse.

\subsubsection{Thermal Infrared Data Analysis}

Thermal data was first subjected to smoothing and enhancement, as described in Chapter 4. This data was then subjected to region identification as described in Chapter 5. These ROIs were chosen based on previous work and/or anatomical orientation of vasculature (or liquid flow in the simulation). The proximal pulse rate and pulse waveform experimentation group consisted of data capture of subject faces. The extremities pulse rate and pulse waveform experimentation group consisted of data capture of subject feet and mechanical blood flow simulation model. For subject feet, the regions were chosen based on the anatomy of the foot; areas close to exposed veins were favored and bony prominences were avoided. The pulse abnormalities experimentation group consisted of data capture of subject's arms as a blood pressure cuff occluded and relieved blood flow.

Following image enhancement and ROI identification, adaptive Eulerian processing was completed for thermal videos as described in Chapter 6. The parameters used for wide temporal EVM processing of thermal and optical perfusion videos are detailed in Table 7.1. The upper and lower bounds of the wide passband were chosen to include a range of normal heart rates. 
Table 7.1 Wide EVM parameter values for all thermal infrared video

\begin{tabular}{|c|c|}
\hline Parameter & Thermal Infrared Video \\
\hline \hline Number of spatial levels & 6 \\
\hline Low frequency bound $(\mathrm{Hz})$ & 0.5 \\
\hline High frequency bound $(\mathrm{Hz})$ & 1.5 \\
\hline Magnification Factor & 100 \\
\hline Frame rate $(\mathrm{Hz})$ & 60 \\
\hline Attenuation factor & 0.1 \\
\hline
\end{tabular}

For each subject (human and mechanical), and each thermal video, the mean intensity was calculated from within each ROI. A Butterworth lowpass filter (cut off frequency of 2.75Hz) was applied in both the forward and reverse directions, resulting in a correction in the phase shift to zero. The resulting signals were then normalized (between 0 and 1) and compared within each subject. Mean intensity signals from each ROI were compared to determine the ROI resulting in a signal best correlated with ECG. The mean intensity signals resulting from the thermal video were also compared to the mean intensity signals resulting from the optical video and respective subject ECG signals to identify and validate a targeted ECG frequency range. A targeted ECG range is considered in this chapter to be a range of 15 beats per minute $(0.25 \mathrm{~Hz})$ centered at the frequency of the mean intensity signal resulting from EVM using a wide passband [20]. The power spectral density of the mean intensity signal resulting from EVM processing using a wide temporal filter was examined to identify the highest amplitude. A frequency range was defined around the highest amplitude for the adaptive EVM step; the application of EVM processing using the same spatial filtering but a targeted, narrower temporal filter, and a higher magnification factor. The parameters for the adapted narrow temporal EVM processing were chosen based on frequency analysis of the previous, wide temporal EVM processing results, and applied to complete the adaptive Eulerian processing. 
The adaptive EVM processing was applied, for which the narrow temporal bandpass filter range was identified in the previous step, and a higher magnification factor was determined from previous work [20]. This targeted EVM processing was completed for all thermal video data. The mean intensity processing was repeated on all resulting enhanced thermal videos using the targeted ROIs. In addition to EVM processing, the original thermal videos were examined at the image enhancement stage; having been subjected to image enhancement only. The ROI mean intensity signals were then calculated from the filtered thermal videos. The results are presented in Section 5.5 and discussed in Section 5.6.

The adaptive EVM analysis aims to enhance temporal variations in video data within a specified frequency range. This method was used for the extraction of the pulse waveform proximal to the body's core, as well as in the extremities. In further analysis of pulse waveform, and the examination of pulse waveform abnormalities, image segmentation is used to examine the specific temperature patterns of blood vessels, as opposed to the general pattern of blood flow and perfusion through an area of skin. The following describes the data analysis methods used for image segmentation of blood vessels subjected to occlusion and relief of blood flow.

Following image segmentation, each output frame from each method was binarized, summed and divided by the number of image pixels. This sum represents the number of pixels in the foreground, and the ratio represents the foreground area percentage. This measurement was examined over time for each method and compared.

\subsubsection{Visible Light Data Analysis}

All visible-light video were subjected to adaptive EVM processing, which was used to measure biological signals without the risk of amplifying noise instead of a biological signal. The 
adaptive EVM approach first employs a wide bandpass filter with a low amplification factor to identify an underlying signal, then re-processing, employing a narrow bandpass filter with a higher amplification factor to reveal a signal representative of subject heart rate, not amplified noise. The EVM method and Adaptive EVM method is described in detail in Chapter 3. The following describes the extraction of signals representative of perfusion rates from Eulerian enhanced visible-light video.

For all human and simulation trials, each resulting visible-light video was subjected to EVM using a wide passband with parameters specified in Table 7.2. The parameters were chosen based on the work of Bennett et. al. [20]. The spatial pyramid was created using 6 levels and a magnification factor of 100 was chosen for a higher Signal to Noise Ratio (SNR). The visible-light video was collected and processed in RGB.

Table 7.2 Wide EVM parameter values for all visible light video

\begin{tabular}{|c|c|}
\hline Parameter & Visible Light Video \\
\hline \hline Number of spatial levels & 6 \\
\hline Low frequency bound (Hz) & 0.5 \\
\hline High frequency bound (Hz) & 1.5 \\
\hline Magnification Factor & 100 \\
\hline Frame rate (Hz) & 60 \\
\hline Attenuation factor & 0.1 \\
\hline
\end{tabular}

Several Regions of Interest (ROIs) were created to determine if the correct heart rate could be extracted and if so, where the ideal ROI would be. Five ROIs were created; one enclosing the entire face, another enclosing the subject's right cheek, another enclosing the subject's forehead and the last two enclosing an area on the subject's chest and arm, respectively. These ROIs are depicted in Chapter 5, Figure 5-11. 
The enhanced video was examined for color intensity within each ROI. The minimum, mean and maximum intensities were found for every frame within each ROI. A Butterworth lowpass filter (cut off frequency of $2.75 \mathrm{~Hz}$ ) was applied in both the forward and reverse directions, resulting in a correction in the phase shift to zero. The resulting signals were then normalized (between 0 and 1) and examined.

The filtered mean intensity signals were compared amongst each other and with the ECG signal. The best performing filtered mean intensity signal was chosen to be the signal that best correlated with the ECG signal. This signal resulted from the ROI enclosing the subject's chest. The filtered mean intensity signals resulting from the same ROI in the light and thermal videos were compared to ensure accuracy.

The original visible-light video was subjected again to EVM, this time using a narrow bandpass filter and a higher magnification factor. The narrow bandpass was chosen to be within the former wide passband, and to surround the frequencies extracted from the subject's chest, using the wide passband. The narrow passband enhanced videos were examined as before. The chest ROI mean intensity signal was found, filtered and compared to the ECG signal to ensure accuracy.

\subsection{Results}

The results are presented for each set of experiments: the simulation pulse measurement experiments, proximal pulse measurement experiments, the distal pulse measurement experiments and the abnormal pulse measurement experiments. This section details the results in a manner corresponding to the research questions: which are the best methods of extracting proximal pulse measurements, which are the best methods of extracting distal pulse measurements, and how do 
these methods perform in the presence of noise and pulse abnormalities. All results are discussed in the following section.

\subsubsection{Simulation Pulse Measurements}

Prior to examining real blood flow in human subjects, this thesis first examined pulse measurements theoretically by capturing thermal and visible-light data of a mechanical system simulating pulsatile blood flow generated by the heart in terms of its frequency and pulse intensity pattern. This experiment aims to strengthen the hypothesis that pulse rate estimation resulting from EVM-enhanced thermal video is due to temperature changes of the skin, and not other physiological phenomenon. Optical and thermal video data were examined here with the intention of determining whether the resulting enhanced signals could be distinguished between being representative of a physiological signal, and not. This was examined by using two modalities to capture data in an instance in which one modality is known to fail; visiblelight cameras are unable to capture temperature changes.

The wide temporal EVM processing, ROI identification and resulting mean intensity signals were calculated over time for both thermal and optical videos of all blood flow simulation trials ( 5 trials in total). The results from one trial can be seen in Figure 7.3 and Figure 7.4. Figure 7.3 depicts the mean intensity signal from within the ROI identified in Figure 5.2, for both the original, unprocessed optical video and the wide temporal EVM optical output video. Figure 7.4 depicts the mean ROI intensity signals for the unprocessed thermal video and the wide temporal EVM thermal output video. There is a clear difference in signals; the thermal camera signal indicates an overall increase in intensity due to the addition of hot water in the reservoir, whereas the optical camera is unable to detect the change in temperature. These 
results were consistent across trials; the average frequencies identified by the EVM optical and thermal output videos were $0.9754 \mathrm{~Hz}$ and $0.7251 \mathrm{~Hz}$, respectively, as compared to the actual pump flow rate of approximately $0.7 \mathrm{~Hz}$. The confidence intervals were $(0.9086,1.0422)$ and $(0.6996,0.7506)$, respectively. These confidence intervals show good repeatability with this experiment. The actual pump flow rate is better represented by the EVM enhanced thermal video than the optical counterpart. Frequency domain analysis of these signals support these statistics, demonstrating that the enhanced optical mean intensity signal is representative of enhanced noise, while the enhanced thermal mean intensity signal displays patterns indicative of an underlying signal. This is shown in the power spectral density plot in Figure 7.5. The frequency range of interest for the narrow temporal filter is indicated in red. This narrower frequency range was chosen to include the highest amplitudes within the frequency range of interest $(0.5 \mathrm{~Hz}-1.5 \mathrm{~Hz})$

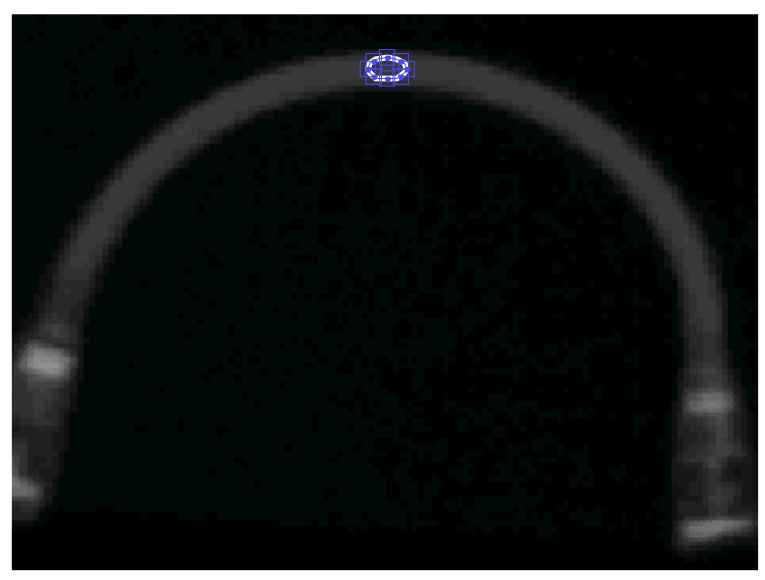

Figure 7.2 Blood simulation model ROI 


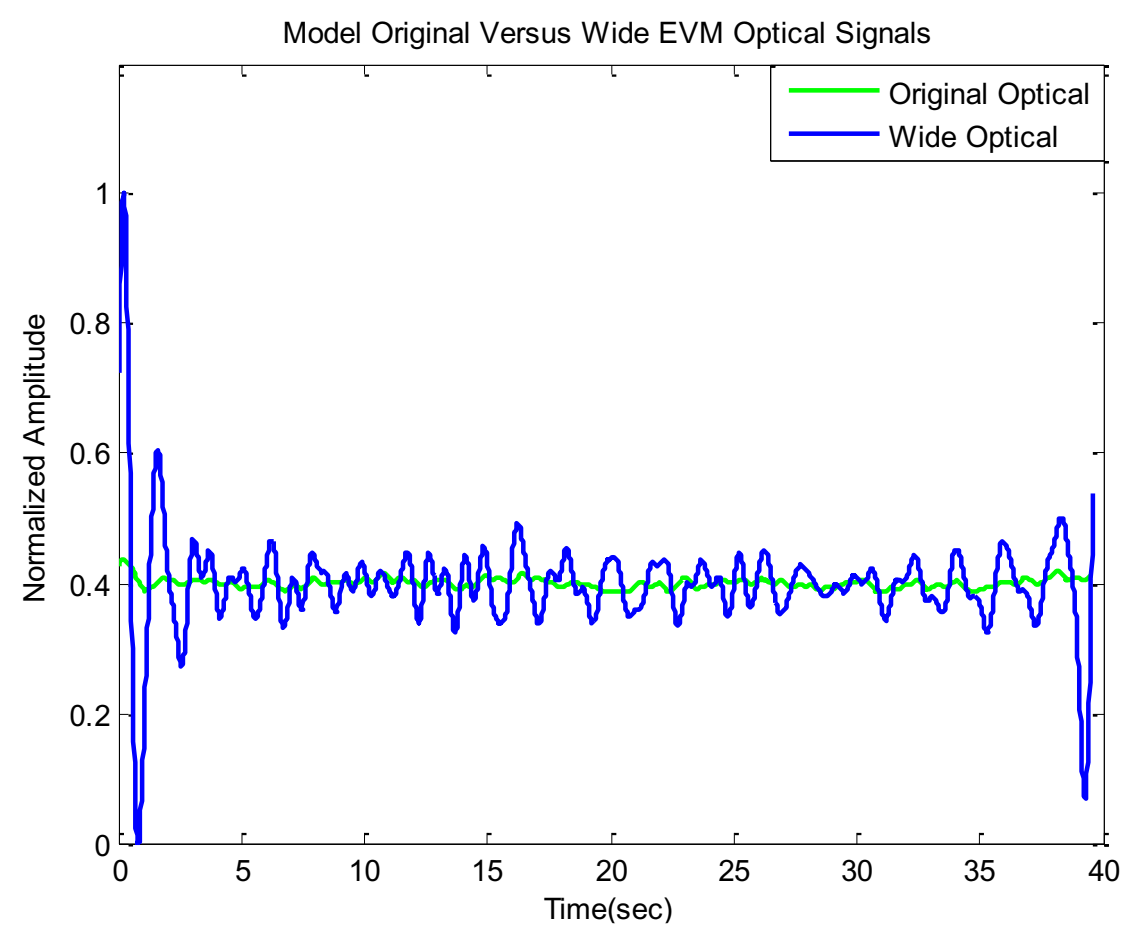

Figure 7.3 Wide, Eulerian enhanced optical mean intensity signal.

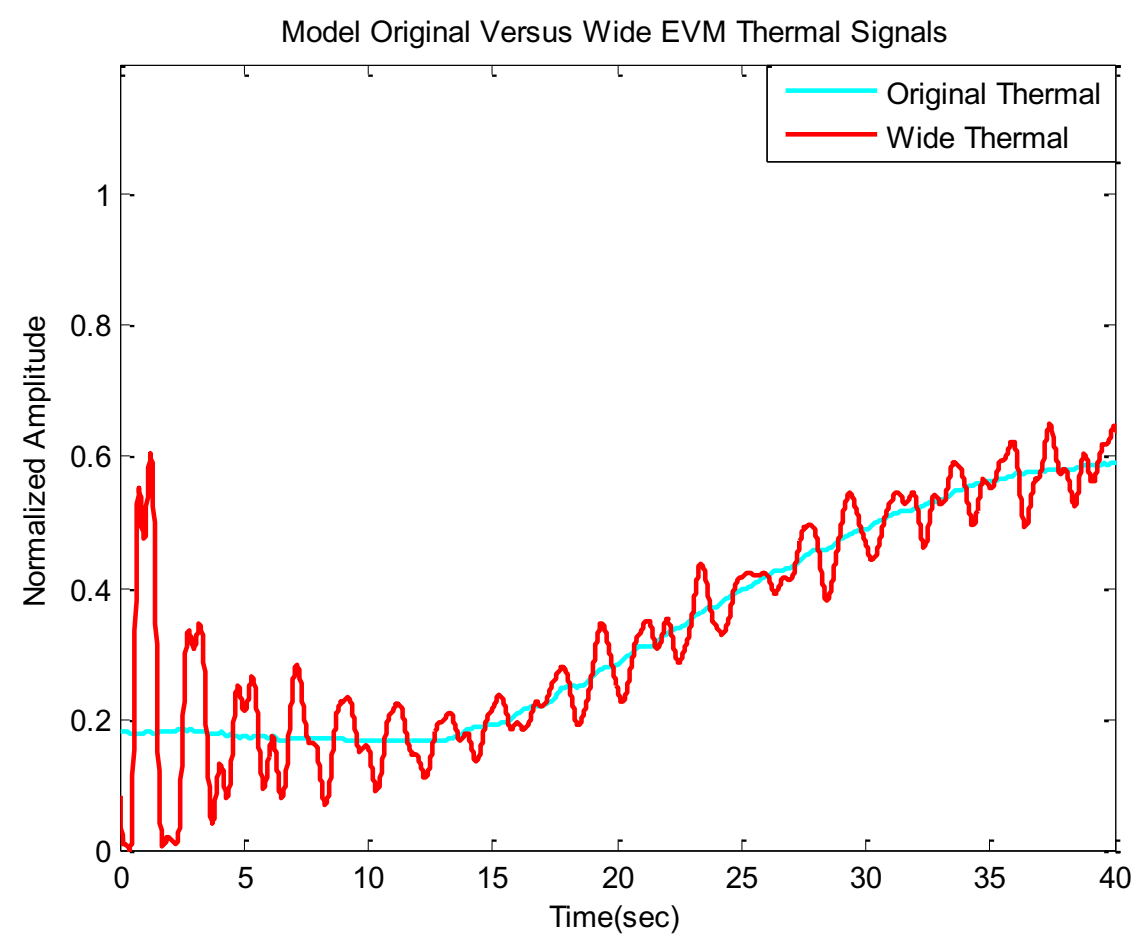

Figure 7.4 Wide, Eulerian enhanced thermal mean intensity signal. 
The narrow EVM processing and mean intensity signals were calculated; the parameters for which can be seen in Table 7.3, and resulting signals for which can be seen plotted in Figure 7.6. The enhanced thermal mean intensity signal is plotted with the unprocessed thermal mean intensity signal and the flow rate of the pump. The signals display similar periodicities, resulting in an error rate of $3 \mathrm{bpm}$. Discussion and conclusions regarding these results are found in the following sections of this chapter.

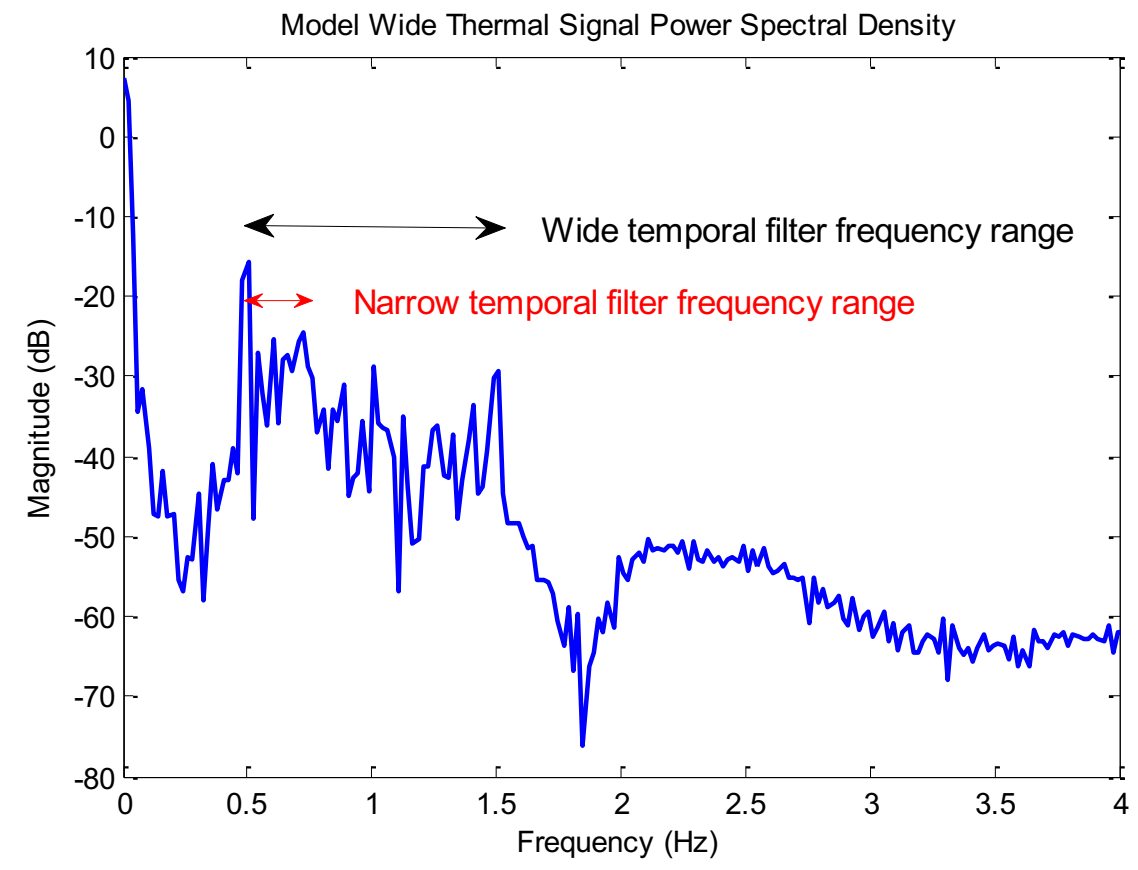

Figure 7.5 Power spectral density plot of the wide temporal Eulerian enhanced thermal mean intensity signal.

Table 7.3 Narrow EVM parameter values for thermal video of the blood flow simulation model

\begin{tabular}{|c|c|}
\hline Parameter & Visible Light Video \\
\hline \hline Number of spatial levels & 6 \\
\hline Low frequency bound $(\mathrm{Hz})$ & 0.50 \\
\hline High frequency bound $(\mathrm{Hz})$ & 0.75 \\
\hline Magnification Factor & 200 \\
\hline Frame rate $(\mathrm{Hz})$ & 60 \\
\hline Attenuation factor & 0.1 \\
\hline
\end{tabular}




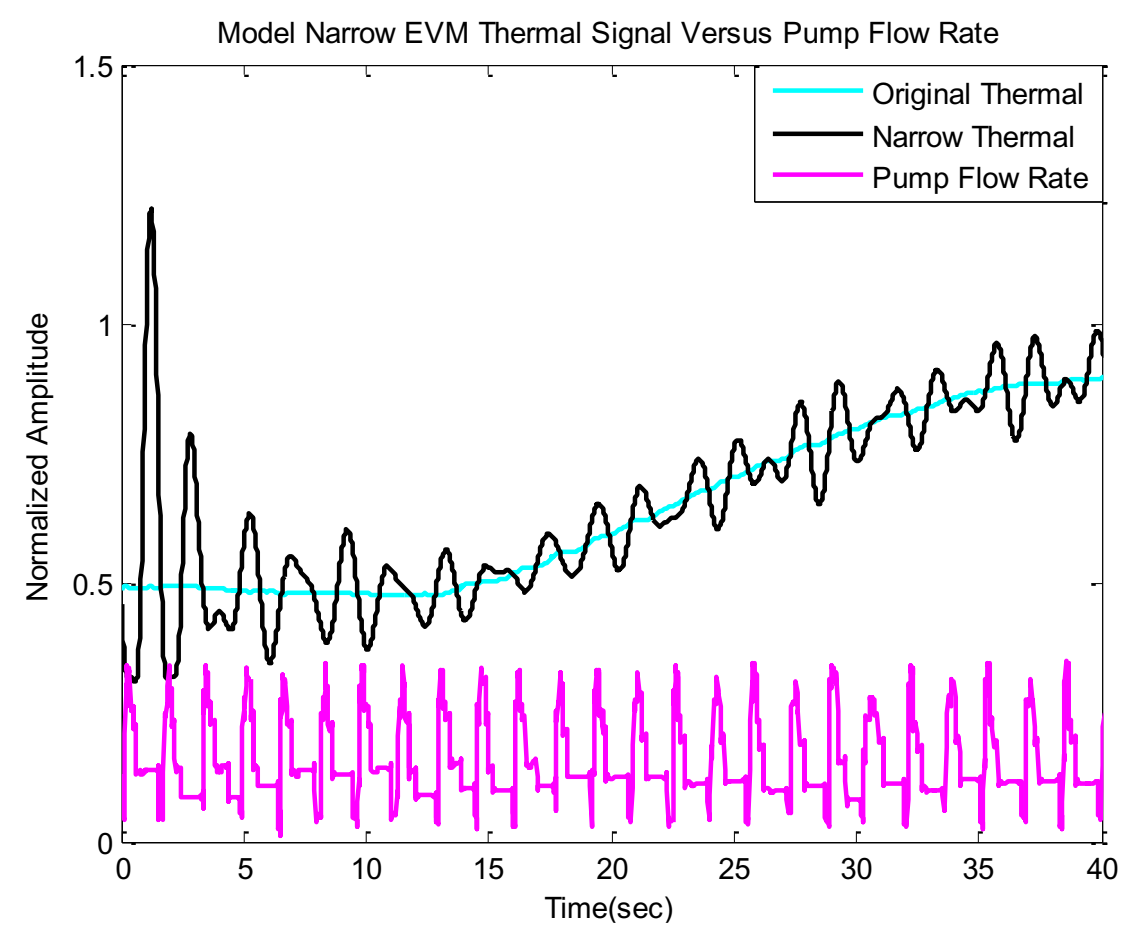

Figure 7.6 Narrow temporal Eulerian enhanced thermal mean intensity signal verses pump flow rate.

\subsubsection{Proximal Pulse Measurement}

Following the examination of simulated pulse measurements, blood flow in human subjects was examined. First, in proximity to the heart. For each subject, all facial perfusion videos were subjected to adaptive EVM processing, and ROI mean intensity signal calculation. The ROI resulting in mean intensity signals best correlated with true heart rate was the forehead ROI. In each instance, the wide temporal Eulerian enhanced mean intensity signal successfully indicated a frequency range of interest for the targeted narrow temporal Eulerian processing. The narrow processing resulted in mean intensity signals that correlated well with the true heart rate signal. The identified narrow frequency ranges and measured error (in bpm) are detailed in Table 7.4. Results from each subject (subjects 1 through 5) are depicted in Figure 7.7 through to Figure 7.11, respectively. Each figure illustrates the differences observed between optical 
and thermal results, which are all normalized (between 0 and 1) and plotted at staggered baseline values. In all cases, the wide Eulerian enhanced optical mean intensity signals were used to validate and compare to the wide Eulerian enhanced thermal mean intensity signals. The wide optical mean intensity signal resulting from subject 5, who has the darkest skin color, appears to vary in both amplitude and frequency, as opposed to the wide optical mean intensity signal resulting from subject 1 , who has the lightest skin color, which is more consistent in amplitude and frequency.

Table 7.4 Narrow EVM parameter values for thermal video of all subjects

\begin{tabular}{|c|c|c|c|c|c|}
\hline \multirow{2}{*}{ Parameter } & \multicolumn{5}{|c|}{ Subject Number } \\
\cline { 2 - 6 } & $\mathbf{1}$ & $\mathbf{2}$ & $\mathbf{3}$ & $\mathbf{4}$ & $\mathbf{5}$ \\
\hline \hline Number of spatial levels & 6 & 6 & 6 & 6 & 6 \\
\hline Low frequency bound (Hz) & 1.08 & 0.83 & 0.83 & 1.08 & 1.08 \\
\hline High frequency bound (Hz) & 1.33 & 1.08 & 1.08 & 1.33 & 1.33 \\
\hline Magnification Factor & 200 & 200 & 200 & 200 & 200 \\
\hline Frame rate (Hz) & 60 & 60 & 60 & 60 & 60 \\
\hline Attenuation factor & 0.10 & 0.10 & 0.10 & 0.10 & 0.10 \\
\hline Error (bpm) & 1 & 3 & 2 & 3 & 6 \\
\hline
\end{tabular}




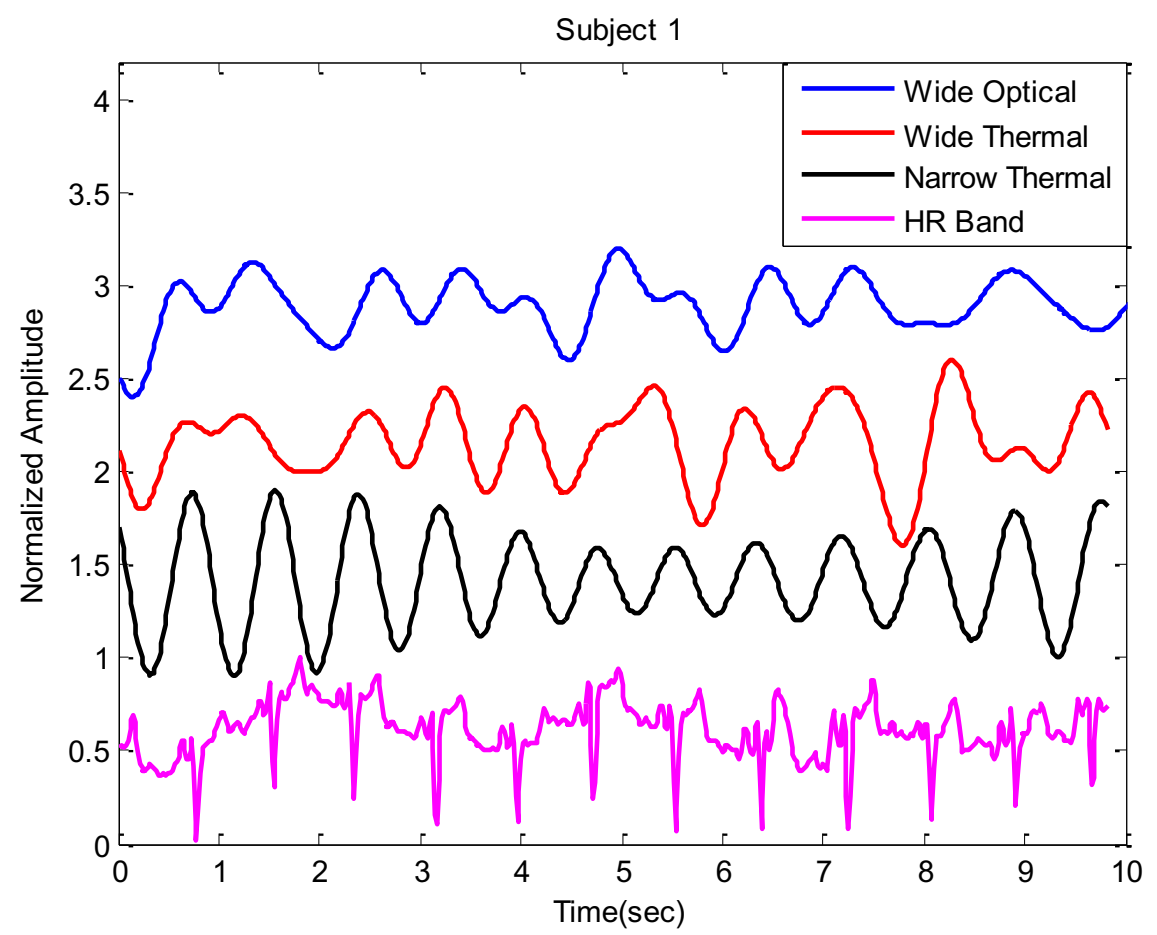

Figure 7.7 Subject 1 Eulerian enhanced mean intensity signals versus heart rate.

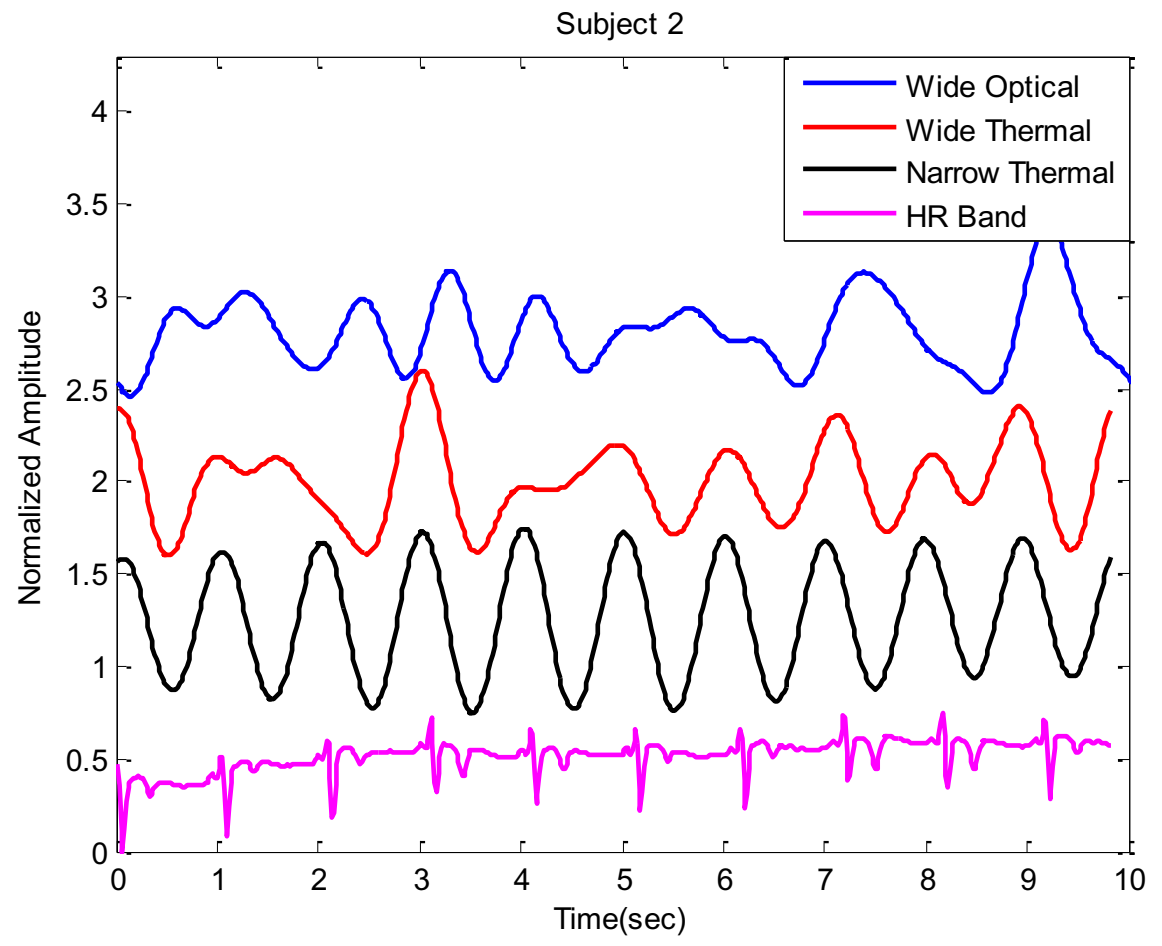

Figure 7.8 Subject 2 Eulerian enhanced mean intensity signals versus heart rate. 


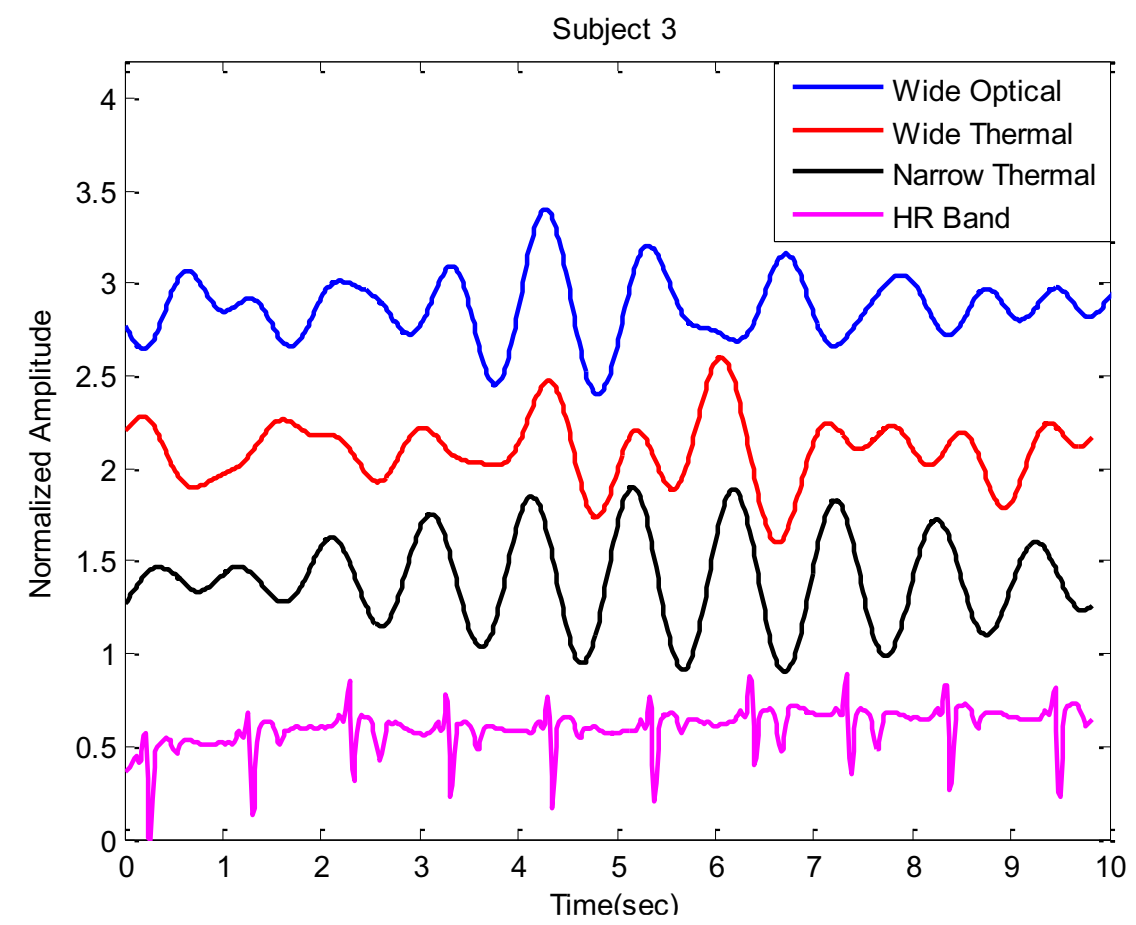

Figure 7.9 Subject 3 Eulerian enhanced mean intensity signals versus heart rate.

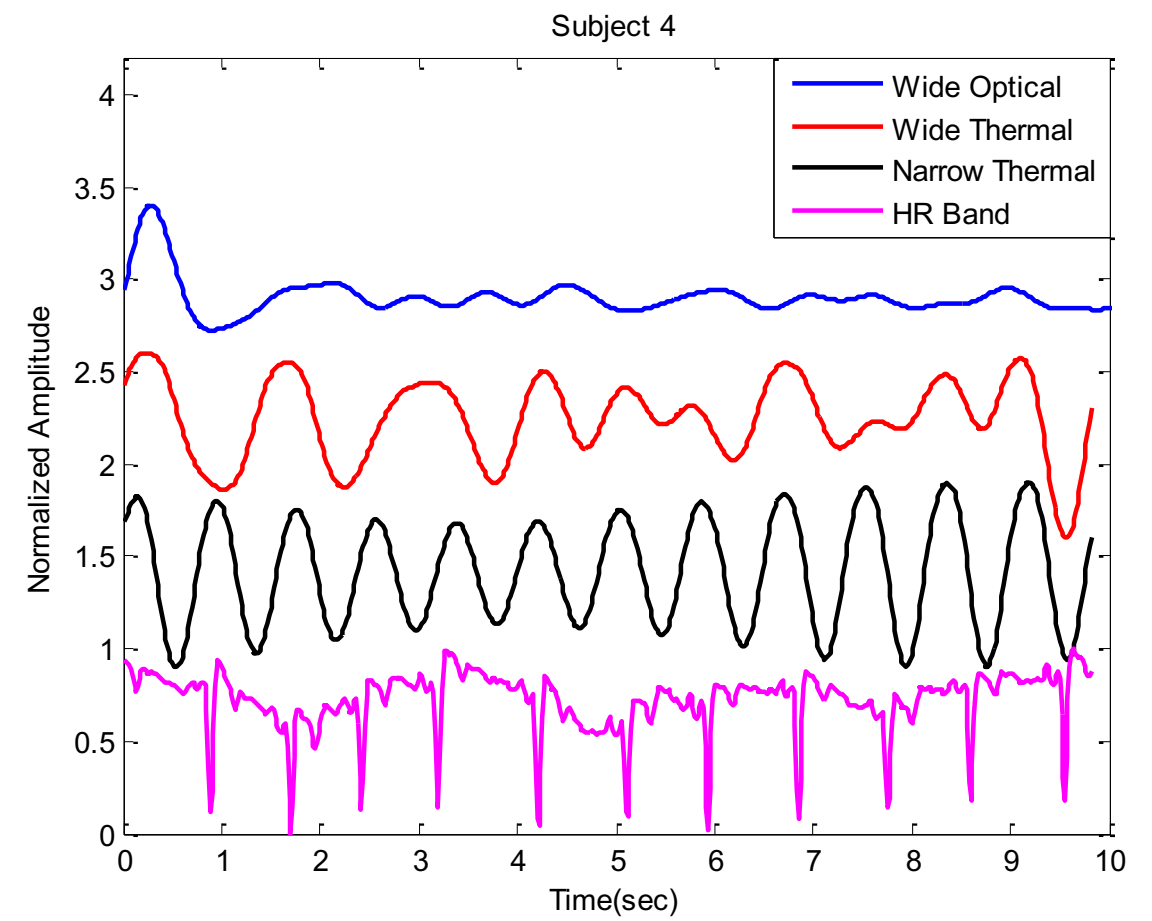

Figure 7.10 Subject 4 Eulerian enhanced mean intensity signals versus heart rate. 


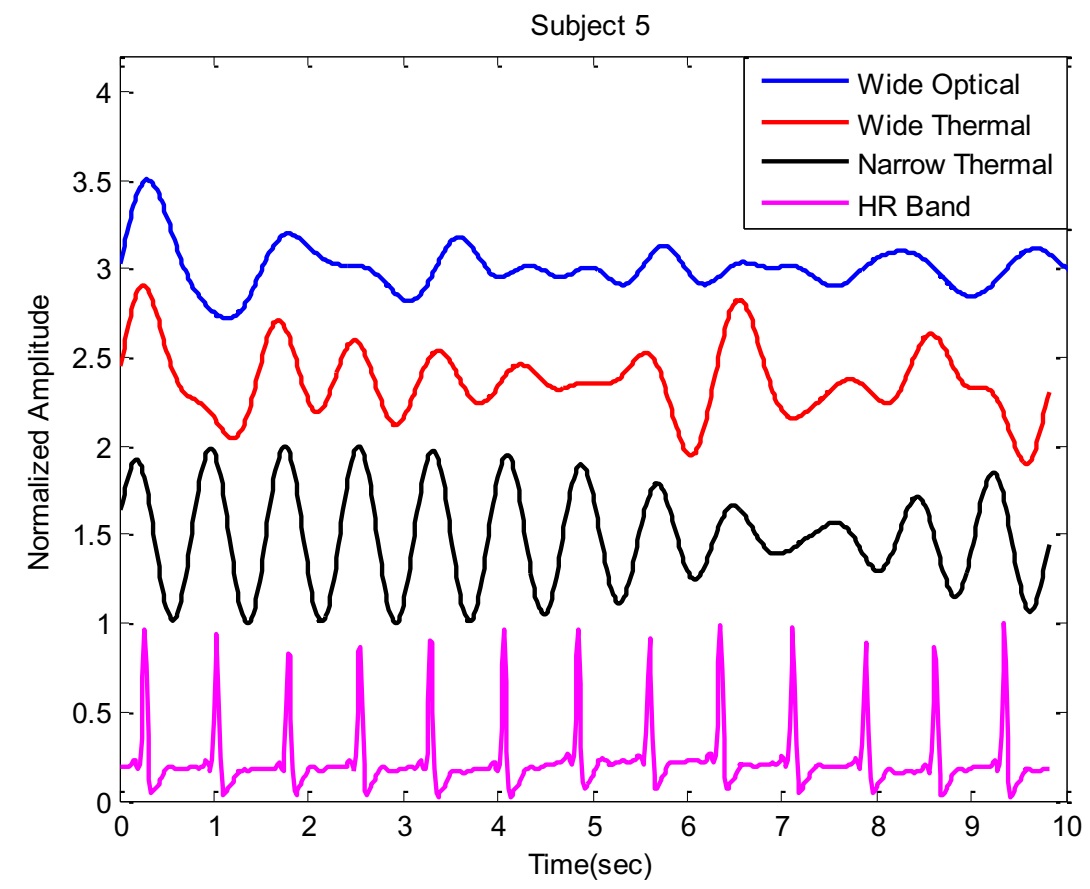

Figure 7.11 Subject 5 Eulerian enhanced mean intensity signals versus heart rate.

\subsubsection{Distal Pulse Measurement}

Blood flow at the extremities of human subjects was then examined; particularly blood flow to the feet. The EVM processing successfully enhanced temporal variations in color representing temperature. Following EVM processing, the healthy adult and patient's output videos were subjected to post-processing. Five ROIs are depicted in Figure 5.6, and were examined for the healthy subject; the mean intensity within each ROI was found temporally. As determined by region analysis in Chapter 5, region 1 produced a mean intensity signal best representing pedal pulse (best corresponding to Doppler measurements). The filtered mean intensity signal resulting from region 1 can be seen plotted against the Doppler detected pulse in Figure 7.13. Both signals have been normalized (between 0 and 1) and are plotted at staggered baseline values. 


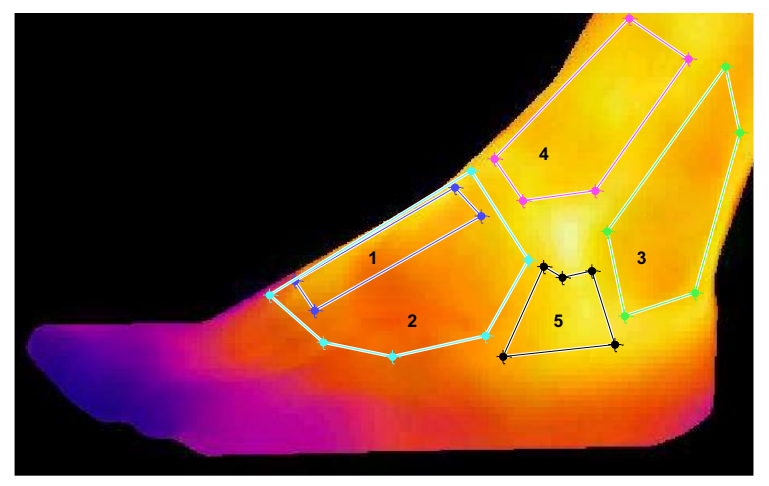

Figure 7.12 Regions of interest on the healthy adult's lateral side of the foot.

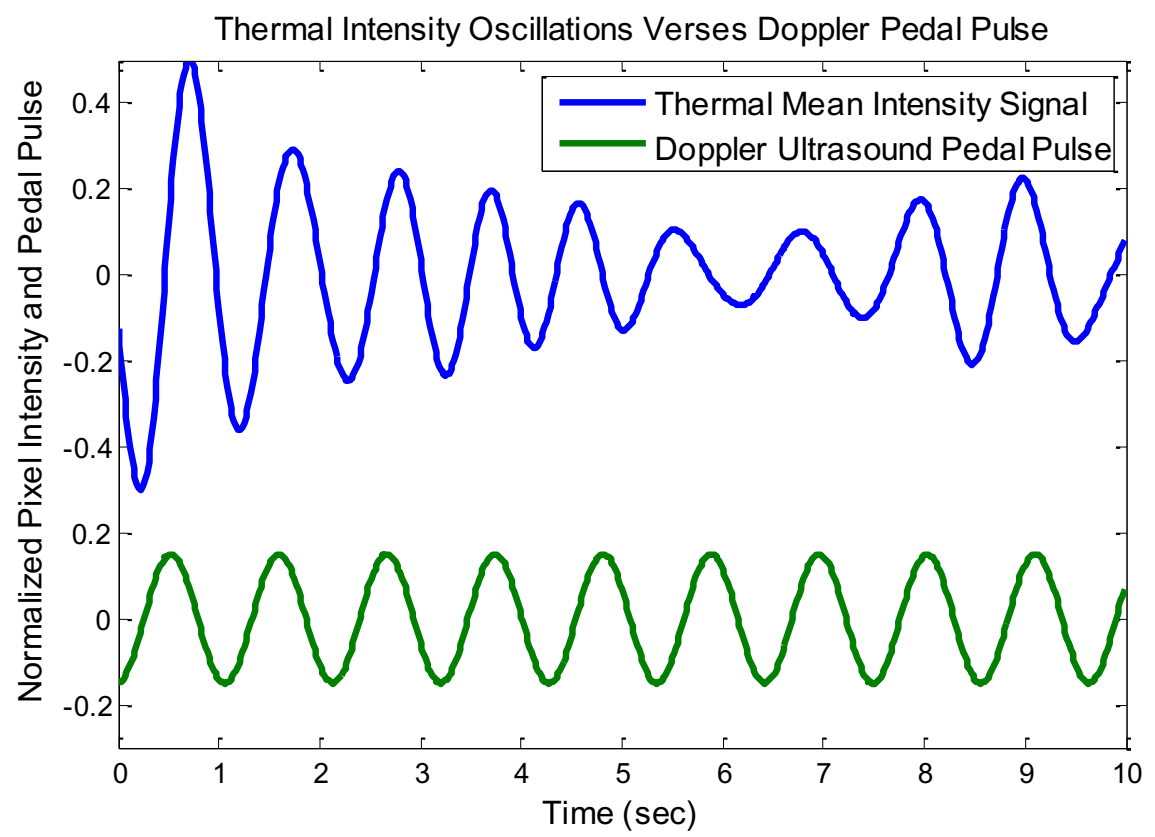

Figure 7.13 Healthy adult region 1 mean intensity signal plotted against the Doppler signal.

The patient's output videos were examined using the mean intensity signal calculated within each of six ROIs (depicted in Figure 5.7). As determined by region analysis detailed in Chapter 5, regions 4 and 5 presented with the largest difference in mean intensity signals of the same frequency. Regions 4 and 5 enclosed the posterior tibial artery on the left and right feet (behind the medial malleoli). The signal resulting from the region enclosing the left foot posterior tibial artery was much weaker than from the right. In other words, the weaker signal 
was on top of the artery supplying blood to the colder heel. These signals can be seen plotted in Figure 7.15.

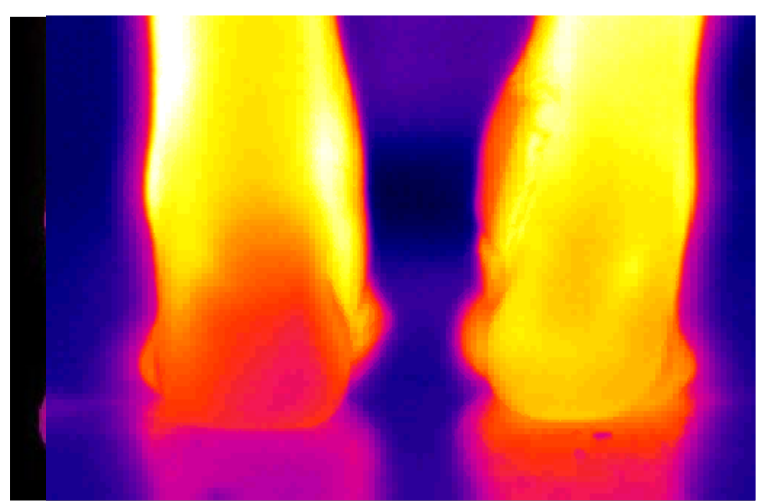

Figure 7.14 Regions of interest on the patient's heels.

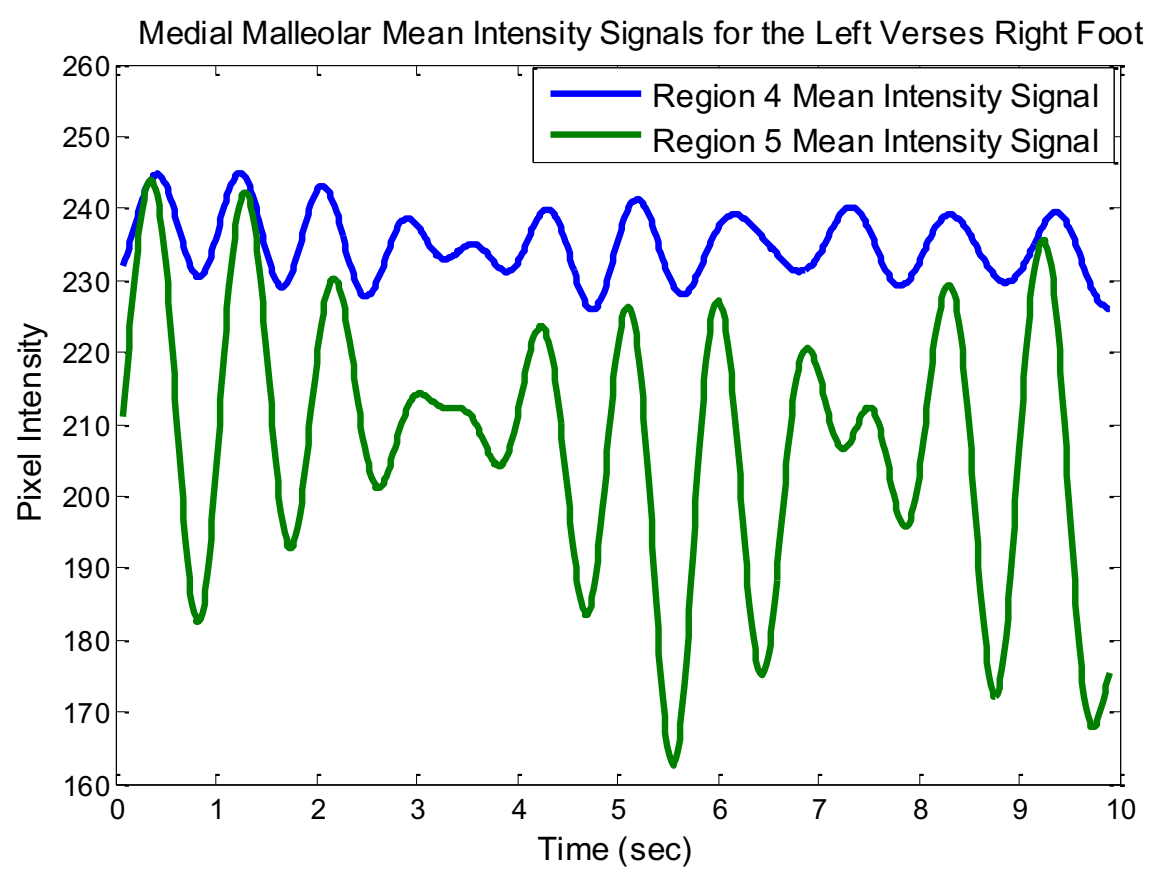

Figure 7.15 Patient's right medial malleolar region plotted against the left. 


\subsubsection{Pulse Abnormalities Measurements}

This thesis then examined pulse measurements in the presence of signal abnormalities; in this chapter the abnormality is introduced via a blood pressure cuff that occludes blood flow. This physical phenomenon is examined through mid-range thermal camera data, EVM processing and ROI mean intensity signal calculation.

The wide Eulerian enhanced thermal mean intensity signals were examined in the frequency domain and an underlying signal representative of ECG was not identified. While an underlying ECG signal was not identified, an overall temperature pattern related to blood flow was identified in the noise reduced thermal videos. As the blood pressure cuff began inflating, the thermal mean intensity signals resulting from the ROI enclosing the brachial artery and the ROI enclosing the arteries at the wrist (ulnar and radial) both increased. When the blood pressure cuff reached maximum inflation to occlude blood flow, the thermal mean intensity signals rapidly decreased, then began to recover as the blood pressure cuff deflated, increasing again until the thermal video was terminated. In other words, the temperature dropped when blood was occluded and rose again when blood returned. An example of this pattern can be seen in Figure 14, which depicts the thermal mean intensity signals resulting from both ROIs of one subject's arm in a noise reduced thermal video. The time at which blood was occluded is indicated. 


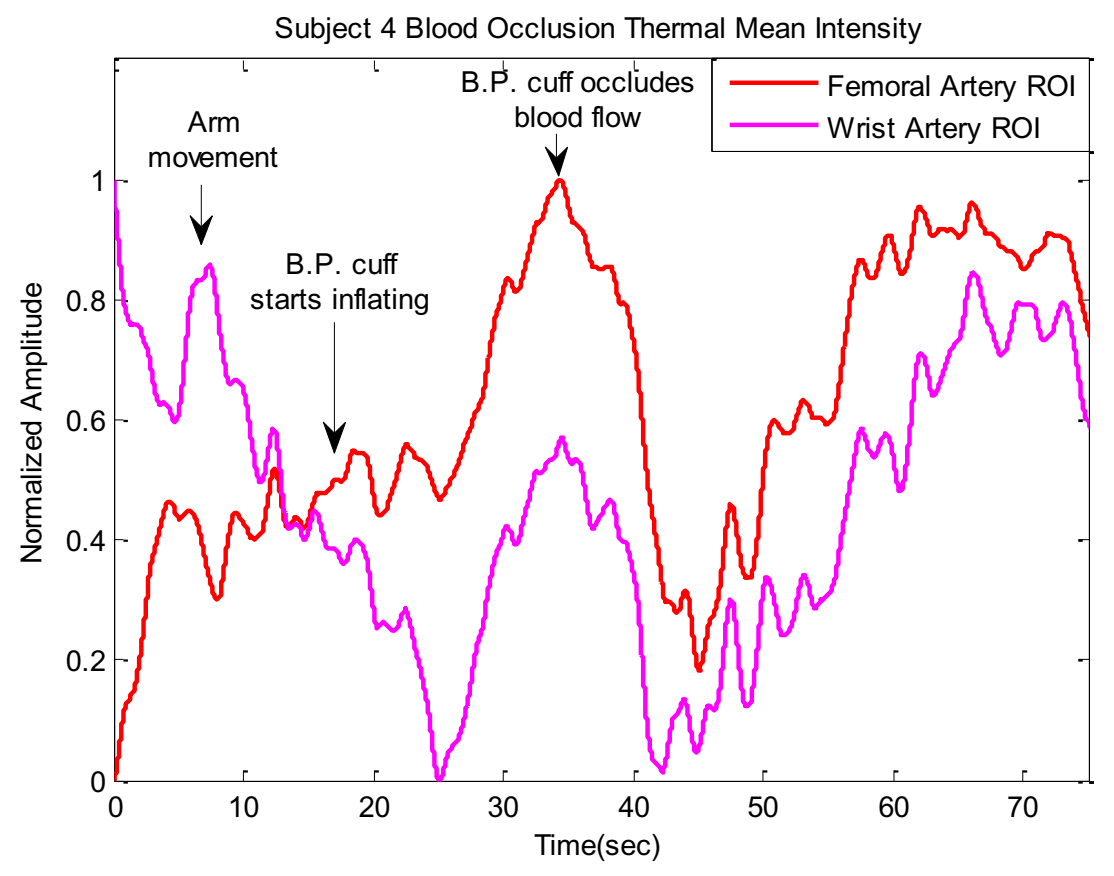

Figure 7.16 Temperature changes at the femoral and wrist regions because of blood occlusion.

Blood occlusion was then captured using a highly sensitive thermal camera with a high thermal resolution. This was to examine specific blood vessels more closely and test the ability of thermal imaging in conjunction with image processing to extract blood flow measurements. A thermal image is depicted in Figure 7.17 reveals the superficial median cubital vein and the comparatively, deeper ulnar artery. This is validated anatomically. Basic image segmentation was first performed on this data using thresholding, an example for which can be seen in Figure 7.18. This was calculated for each frame of the thermal video as the blood pressure cuff operated, respectively. The thresholded images were the input to two other segmentation methods; level-set and watershed methods. Thermal image segmentation analyses and results are detailed in Chapter 5. Following image segmentation, each output frame from each method was binarized, summed and divided by the number of image pixels. This sum represents the number of pixels in the foreground, and the ratio represents the foreground area percentage. 
This measurement was examined over time for each method and compared.

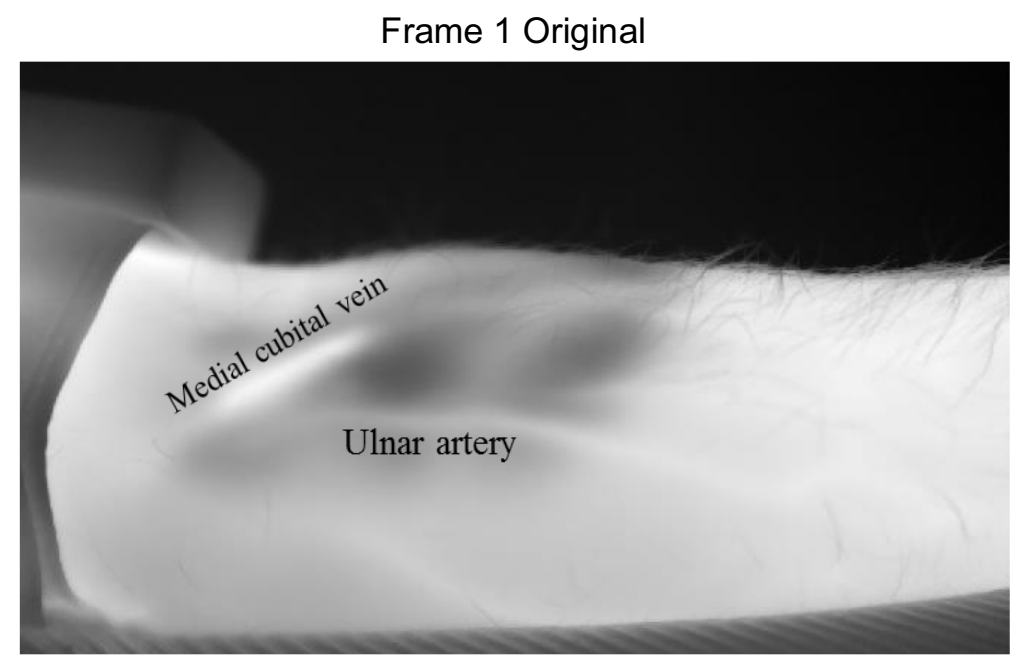

Figure 7.17 Frame 1 original thermal image.

Frame 1 Cropped and Segmented

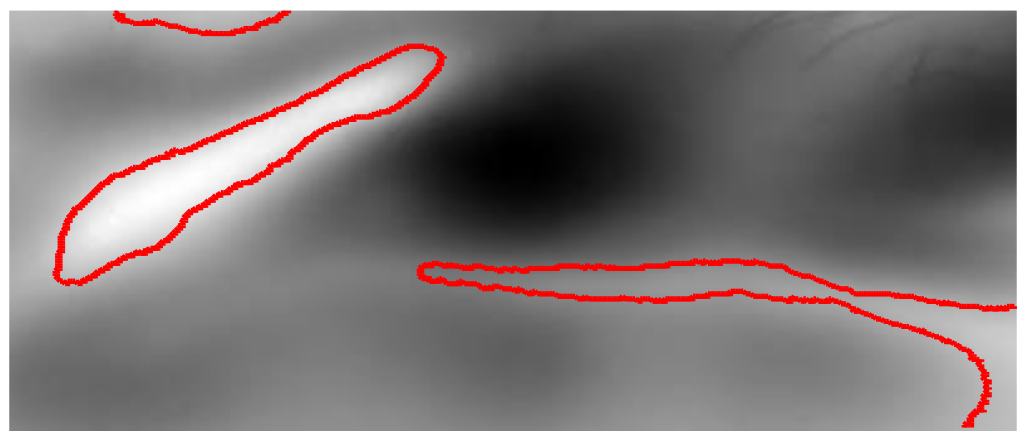

Figure 7.18 Frame 1 cropped and segmented image using basic thresholding.

While the segmentation results varied in region detail, all methods indicate a pattern associated with vascular occlusion in the segmented median cubital vein and the ulnar artery. The median cubital vein appears to maintain its shape and size throughout the experiment, while the ulnar artery indicates regular blood flow, followed by a lack of blood flow (ulnar artery region shrinks in size) when vascular occlusion occurs, and the return of blood flow when the occlusion is relieved. This pattern is expected, as vascular occlusion will prevent 
blood flowing through the arteries to the body (the arteries will drain) and blood flowing back through the veins to the heart (the veins will remain filled). The percentage area measurements over time indicated quantitatively what is observed visually; the overall object size decreases as vascular occlusion occurs and increases again as the vessels recover. This result is seen in Figure 7.19.

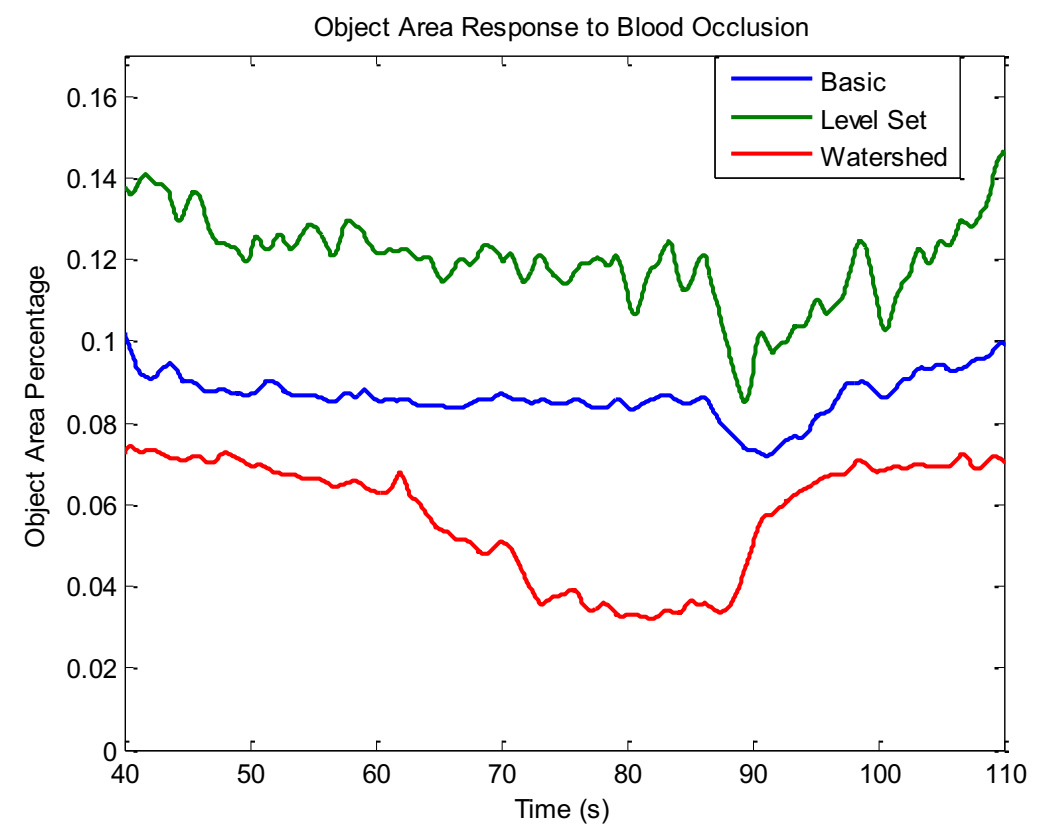

Figure 7.19 Object area percentage measurement over time, extracted via basic thresholding, DRLSE and watershed methods.

\subsection{Discussion}

This section discusses the results from each set of analyses: the simulation pulse measurement, proximal pulse measurement, distal pulse measurement and abnormal pulse measurement analyses. 


\subsubsection{Simulation Pulse Measurements}

The mechanical system was designed to pump water through tubes in a pulsatile manner like that of a heart. This system allowed for the observation and examination of a controlled system of known variables operating periodically like the heart. The effect of EVM processing on thermal video was therefore better examined and validated. Hot water was added to the system while thermal and optical cameras captured video data. Both thermal and optical video data were subjected to ROI selection and AEVM processing. The overall change in temperature was visible and measurable in the thermal video, but undetectable in the optical video. The adaptive EVM processing of thermal video successfully enhanced the changes in temperature due to periodic pulsing, whereas the adaptive EVM processing of optical video produced enhanced noise. These results were consistent in repeated simulations, and validated the use of adaptive EVM processing to extract mean intensity signals representative of flow rate in a controlled system. These results also demonstrated and identified an instance in which adaptive EVM processing enhanced noise and not an underlying signal, (optical cameras do not detect temperature). The simulation experiment therefore helped to characterize a successful and unsuccessful Eulerian enhancement. Eulerian enhancement is discussed with respect to noise in Chapter 6 as well as in this chapter.

\subsubsection{Proximal Pulse Measurements}

The blood perfusion experiments with human subjects included facial perfusion experiments as a proximal perfusion measure. Facial perfusion data were subjected to ROI selection and adaptive EVM processing. The adaptive EVM processing resulted in the successful enhancement of mean intensity signals representative of perfusion rate, determined visually 
and using calculated error with ECG signals. There was a notable difference between the mean intensity signals resulting from applying adaptive EVM processing to thermal versus optical video. Optical video in conjunction with EVM is known to be sensitive to lighting conditions

and skin color [2]. This was observed in the facial perfusion results, while also observing that thermal video in conjunction with EVM is not sensitive to these variables. These results suggest that, unlike optical video, thermal video subjected to adaptive EVM processing may reveal signals representative of perfusion rate, independent of skin color or lighting conditions. This study does not address subject movement; a major obstacle in detecting heart rate. Extensive work has been done in object tracking, particularly in optical videos [20],[131]-[133]. Facial recognition and object tracking can be added to image processing techniques, to tackle subject movement.

\subsubsection{Distal Pulse Measurements}

This study subjected thermal videos of the lateral side of a healthy adult's foot to EVM and examined the output videos in relation to the simultaneously captured Doppler ultrasound pedal pulse signal. The EVM processing successfully enhanced temporal intensity changes in the thermal video representative of temperature changes. Five ROIs were defined and the mean intensity was calculated for each ROI. Regions 1 and 2 mean intensity signals displayed the same frequency patterns as the Doppler ultrasound signal. It is interesting to note that regions 1 and 2 both contain the Dorsalis Pedis Artery, which is superficial and runs along the top of the foot. It is even more interesting to note that region 1, the region resulting in the strongest signal amplitude, is directly on top of and area-limited to the Dorsalis Pedis Artery. If the desired signal is detected at the arteries, the Signal Noise Ratio (SNR) is high at these areas. Conversely, the SNR is lower in other areas, resulting in amplified noise. These results suggest 
that thermal video, in conjunction with EVM processing can be used to extract a signal representative of pedal pulse, specifically from areas where arteries are close to the skin (areas of high SNR).

This thesis then subjected thermal videos of a diabetic, dialysis patient's heels (posterior view) to EVM and examined the output videos for patterns. The examined patient has one significantly colder heel (the left heel), and so these signals were examined specifically for patterns indicative of microvascular health. The EVM processing successfully enhanced the temporal intensity variations and mean intensity signals were extracted from six ROIs. Half of the ROIs oscillated at $1.1 \mathrm{~Hz}$, while the other half oscillated at $1.2 \mathrm{~Hz}$. The largest difference between two mean intensity signals was between regions 4 and 5 . These are interesting results considering the anatomy of the foot. The posterior tibial artery runs down the calf on the medial side, behind the medial malleolus and then towards the toes, underneath the arch of the foot. This artery supplies blood to the heel and is closest to the skin at the medial malleolus. The results indicate that the largest difference in mean intensity signal amplitudes was between the areas enclosing the posterior tibial artery on the left and right feet (behind the medial malleolus). The mean intensity signal resulting from the region enclosing the left foot posterior tibial artery was much weaker than from the right. In other words, the weaker signal was on top of the artery supplying blood to the colder heel. The differences in mean intensity signal frequencies $(1.1 \mathrm{~Hz}$ versus $1.2 \mathrm{~Hz}$ ) may be the result of differences in SNR at these areas. The areas enclosing arteries may have a higher SNR than the areas that do not. Eulerian enhancement in the former instance is likely to have revealed the pedal pulse while the latter group is likely the result of enhanced noise. While the pedal pulse of the patient was not measured at the time of thermal video capture, the healthy adult results strengthen this theory. 


\subsubsection{Pulse Abnormalities Measurements}

Pulse signal abnormalities were examined through mid-range thermal camera data, EVM processing and ROI mean intensity signal calculation, then by means of a highly sensitive thermal camera with a high thermal resolution, and image segmentation. This was to examine overall thermal patterns, and then patterns related to specific blood vessels.

The mid-range blood occlusion data were subjected to ROI selection and adaptive EVM processing. While the blood flow was cut off, as indicated by a lost signal in the pulse oximeter, no underlying signal was detected in the Eulerian enhanced thermal videos. A temperature response due to blood occlusion was detected by the thermal camera (rapid drop in temperature followed by a steady increase in temperature), but not in the pulsatile manner of perfusion. It is possible that a perfusion signal was not detected because arteries in the face are more superficial than in the arm [68]. A highly sensitive thermal camera was then used, to more definitively determine if thermal video in conjunction with EVM can detect and quantify perfusion in the extremities.

Thermal video, of high thermal resolution, of a subject undergoing a blood pressure reading was cropped and binarized using thresholding. The thermal and binary images were compared to anatomical images to confirm the segmented areas were blood vessels. The binary images representing each video frame were the input to watershed and DRLSE image segmentation methods. Region analyses are discussed in Chapter 5, and the temporal signal patterns are discussed in this section. All image segmentation methods indicated the expected pattern associated with vascular occlusion in the segmented median cubital vein and the ulnar artery. This pattern was observed and quantified; the overall object size decreases as vascular 
occlusion occurs and increases again as the vessels recover. This suggests that thermal imaging in conjunction with image segmentation can detect and quantify normal blood flow, and interrupted blood flow.

\subsection{Conclusion}

This chapter focused on the algorithmic processes and outcomes of pulse measurements. Image and signal processing methods detailed in this chapter were used to estimate pulse measurements close to the heart (proximal measurements), pulse measurements at the extremities (distal measurements), and the effectiveness of the proposed methods in the presence of noise and signal abnormalities.

The results of this chapter demonstrated the potential of thermal video in conjunction with adaptive EVM methods to extract an underlying temperature signal representative of subject pulse, both proximally and distally, and detect and distinguish between an underlying signal and the amplification of noise. This chapter also demonstrated some advantages of Eulerian enhanced thermal videos over Eulerian enhanced visible-light videos in the extraction of vital signals; lighting conditions and subject colour have a confounding impact on visible-light data, where thermal data remains impervious. 


\section{Chapter 8}

\section{Respiration Measurement Extraction}

This chapter builds upon the previous chapters by detailing the specific experimentation, data analysis, results and discussion associated with respiration.

\subsection{Introduction}

The entire algorithmic system is depicted in Figure 8.1 below, where this chapter focuses on the processes and outcomes of respiration measurements, the component highlighted orange. The following sections will detail specific image and signal processing methods used to estimate respiration measurements, and the effectiveness of the proposed methods in the presence of movement, noise and signal abnormalities.

This chapter refers to several image processing methods detailed in previous chapters. This includes image smoothing and enhancement, detailed in Chapter 4, Region of Interest identification, detailed in Chapter 5, and Adaptive Eulerian Video magnification (AEVM), detailed in Chapter 6. 


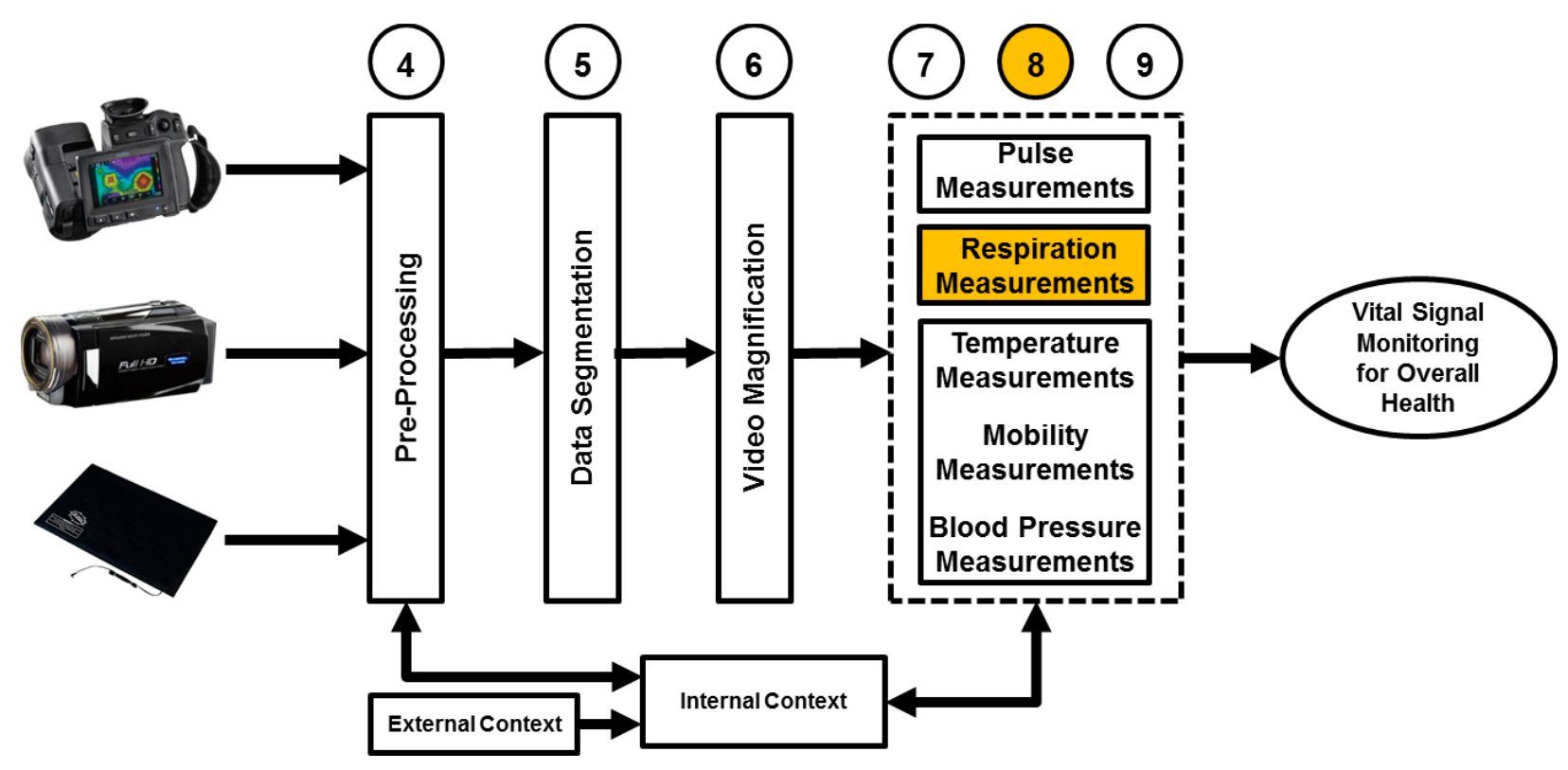

Figure 8.1 Algorithmic system with highlighted chapter focus

\subsection{Equipment and Experimentation}

The equipment used in the experimentation specific to respiration rate measurements were inclusive of two thermal cameras, a biosignal chest strap measuring physiological signals, and a laptop. Technical specifications for all equipment are detailed in Chapter 4.

The thermal infrared cameras were used to gather thermal data from subjects. Throughout experimentation, thermal video data were collected of different areas of the subject's bodies. For respiration rate, experimentation included data collection from subject's faces and upper bodies. All data was collected and analyzed on the laptop.

The biosignal chest strap captures heart rate and breathing rate, among several other physiological signals. The device is worn by the user for the duration of data collection and removed afterwards for data download. The biosignal chest strap was used as the gold standard in data analysis, providing the true measurement of respiration rate. 


\subsection{Respiration Measurement Data Analysis}

This section details the specific algorithms developed in this thesis to extract respiration measurements from the measurement source, the thermal infrared cameras. Respiration measurements in this chapter include the rate at which a subject is breathing, in addition to the shape, rhythm and strength of breaths.

\subsubsection{Thermal Video Data Analysis}

Thermal video data analysis began with image correction and enhancement, followed by region identification and EVM. These processes are detailed in Chapters 3, 4 and 5, respectively. Image correction and enhancement was performed using a median filter and contrast-limited adaptive histogram equalization. EVM was applied to thermal video, and a region of interest was identified as one encompassing both subject nostrils. This section details respiration signal extraction from the subject through intensity measurements as well as through motion measurements.

Intensity measurements were captured by calculating a mean intensity signal from within the identified ROI for every thermal video frame. A Butterworth lowpass filter (cut off frequency of $0.35 \mathrm{~Hz}$ for normal respiration) was applied in both the forward and reverse directions, resulting in a correction in the phase shift to zero. The resulting signals were then normalized (between 0 and 1) and aligned with the RIP band data to validate respiration rates.

Motion measurements were captured by analyzing each thermal video frame for changes relative to the first frame. The first frame was set as the reference frame. The absolute difference image between the reference frame and every succeeding frame was calculated. Each absolute difference frame was binarized using a threshold of 0.1 (as can be seen in Figure 
8.2) and summed to obtain a Sum of Absolute Differences (SAD) measurement. This measurement was calculated for every difference frame, resulting in a SAD signal over time that was lowpass filtered to remove noise, and compared to actual respiration rate. Results from the described image processing for temperature-based and motion-based data analyses are presented in the following section.

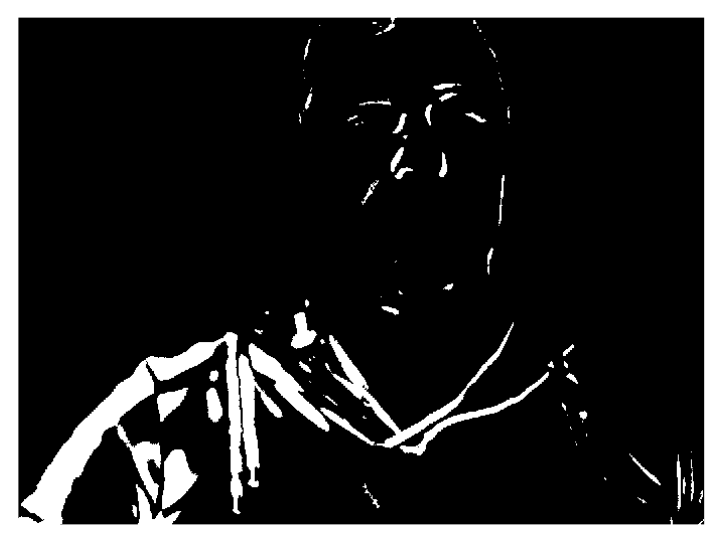

Figure 8.2. Absolute difference image.

\subsection{Results}

The results are presented for each set of measurements and analysis; the normal respiration measurements, and the abnormal respiration measurement experiments. This section details the results in a manner corresponding to the research questions: can normal respiration and abnormal respiration such as sleep apnea be detected and extracted from thermal and visible light video data using the described intensity based and motion based signal extraction methods.

\subsubsection{Normal Respiration Measurement}

The EVM for color enhancement resulted in output videos that successfully enhanced the variations in color over time. Color in this example is representative of temperature, so the output videos displayed enhanced temperature variations over time. This enhancement is 
depicted in Figure 8.3, where each top subplot depicts the subjects' nose in the input video at three different frames; in the first frame the subject is breathing in, breathing out in the second frame and breathing in again in the third frame. The bottom subplot depicts the same three frames of the output video. The magnification of color enhances the change in temperature while breathing. This is particularly evident when the subject is breathing in cool air; the subject's nose becomes darker in color, indicating it has become colder.

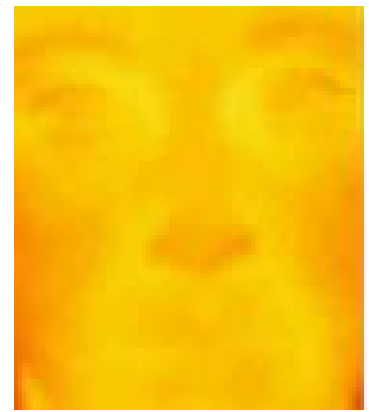

Inhale

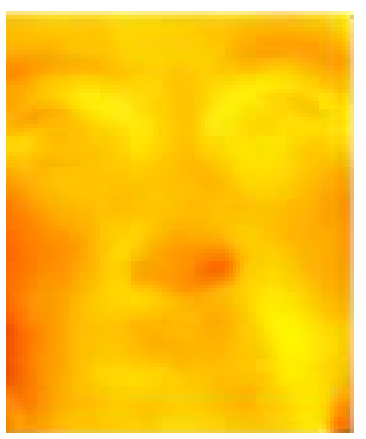

Inhale

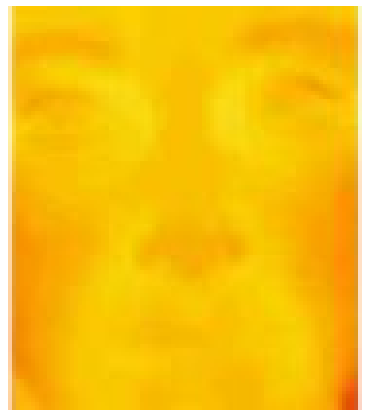

Exhale

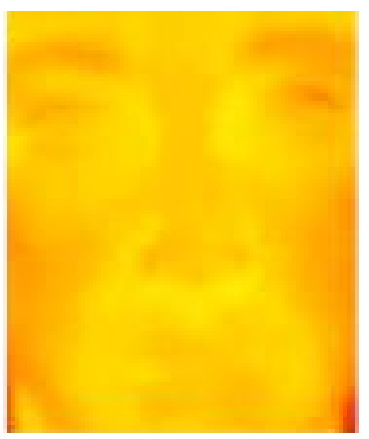

Exhale

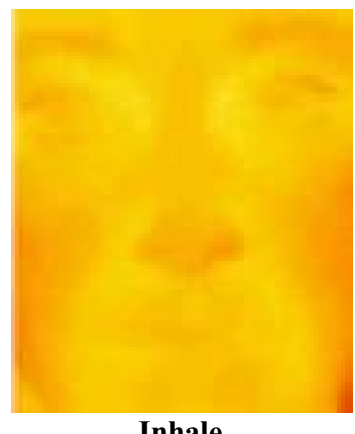

Inhale

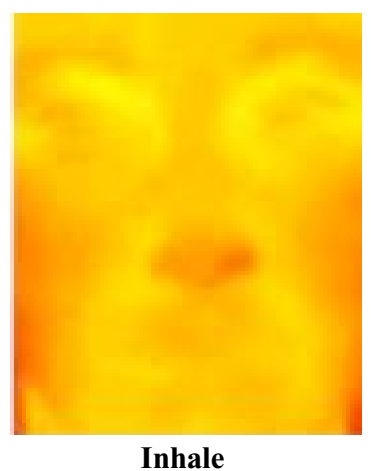

Inhale

Figure 8.3. Three different thermal video frames (top subplot) and the same video frames after Eulerian video magnification (bottom subplot).

The parameters were chosen based on the method described in Chapter 5. The narrow bandpass filter was chosen with the frequencies of interest in range; approximately $0.15 \mathrm{~Hz}$ for normal breathing. A magnification factor of 10 was used for all trials. The final parameter values 
chosen for Eulerian color magnification of thermal video for the enhancement of respiratory behavior, are displayed in Table 8.1 .

Table 8.1 Final EVM Parameter Values Normal Breathing

\begin{tabular}{|c|c|}
\hline Parameter & Normal Breathing \\
\hline Low frequency bound of the band-pass filter & $0.1 \mathrm{~Hz}$ \\
\hline High frequency bound of the band-pass filter & $0.2 \mathrm{~Hz}$ \\
\hline Magnification Factor & 10 \\
\hline Frame rate & $60 \mathrm{~Hz}$ \\
\hline Attenuation factor & 0.1 \\
\hline
\end{tabular}

The mean intensity signal at the nostrils was a good representation of the temperature oscillations related to breathing. The first breathing tests were to extract normal nasal respiration, the results for which are depicted in Figure 8.4. While the region of interest was determined to be the entire nose, as described in Chapter 5, the mean intensity signal was also examined from an ROI enclosing one nostril tightly. In other words, a smaller, more targeted ROI. In both figures, the top plot depicts the normalized (between 0 and 1) mean intensity of the tight left nostril ROI plotted with the respiration rate extracted from the RIP band on a normalized (between 0 and 1) scale. The bottom plots depict the mean intensity of the whole nose ROI plotted with the RIP band signal. By observing the figure, it is visually evident that the extracted signals for normal breathing were detectable using an ROI enclosing one nostril or a larger ROI enclosing the entire nose. 

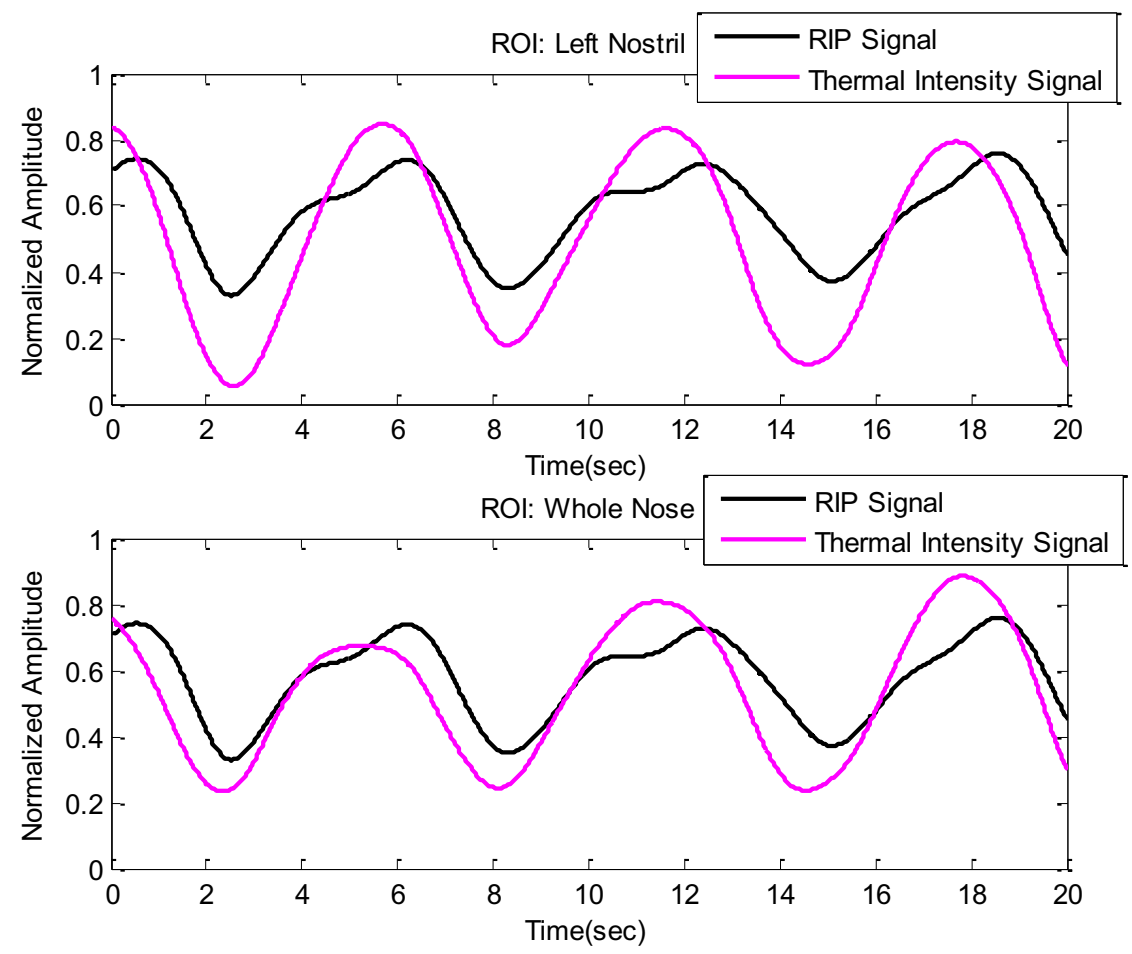

Figure 8.4. Normal respiration intensity values plotted with RIP signals.

\subsubsection{Abnormal Respiration Measurement}

The AEVM for color enhancement resulted in output videos that successfully enhanced the variations in color over time. The AEVM parameters were chosen based on the method described in Chapter 5. The narrow bandpass filter was chosen for both trials considering the frequency of interest; approximately $0.60 \mathrm{~Hz}$ for hyperventilation. A magnification factor of 10 was used for all trials. The final parameter values chosen for AEVM of thermal video for the enhancement of respiratory behavior, are displayed in Table 8.2.

The hyperventilation and OSA simulations results are depicted in Figure 8.5 and Figure 8.6, respectively. In both figures, the top plot depicts the normalized (between 0 and 1) mean intensity of the tight left nostril ROI plotted with the respiration rate extracted from the RIP band on a normalized scale (between 0 and 1). The bottom plots depict the mean intensity of 
the whole nose ROI plotted with the RIP band signal. By observing Figure 8.6, it can be seen that the respiration rates for hyperventilation were detectable using an ROI enclosing one nostril or a larger ROI enclosing the entire nose.

Table 8.2 Final EVM Parameter Values Abnormal Breathing

\begin{tabular}{|c|c|c|}
\hline Parameter & Hyperventilation & $\begin{array}{c}\text { OSA } \\
\text { Simulation }\end{array}$ \\
\hline $\begin{array}{c}\text { Low frequency bound of } \\
\text { the band-pass filter }\end{array}$ & $0.5 \mathrm{~Hz}$ & $0.1 \mathrm{~Hz}$ \\
\hline $\begin{array}{c}\text { High frequency bound of } \\
\text { the band-pass filter }\end{array}$ & $0.7 \mathrm{~Hz}$ & $0.2 \mathrm{~Hz}$ \\
\hline Magnification Factor & 10 & 10 \\
\hline Frame rate & $60 \mathrm{~Hz}$ & $60 \mathrm{~Hz}$ \\
\hline Attenuation factor & 0.1 & 0.1 \\
\hline
\end{tabular}
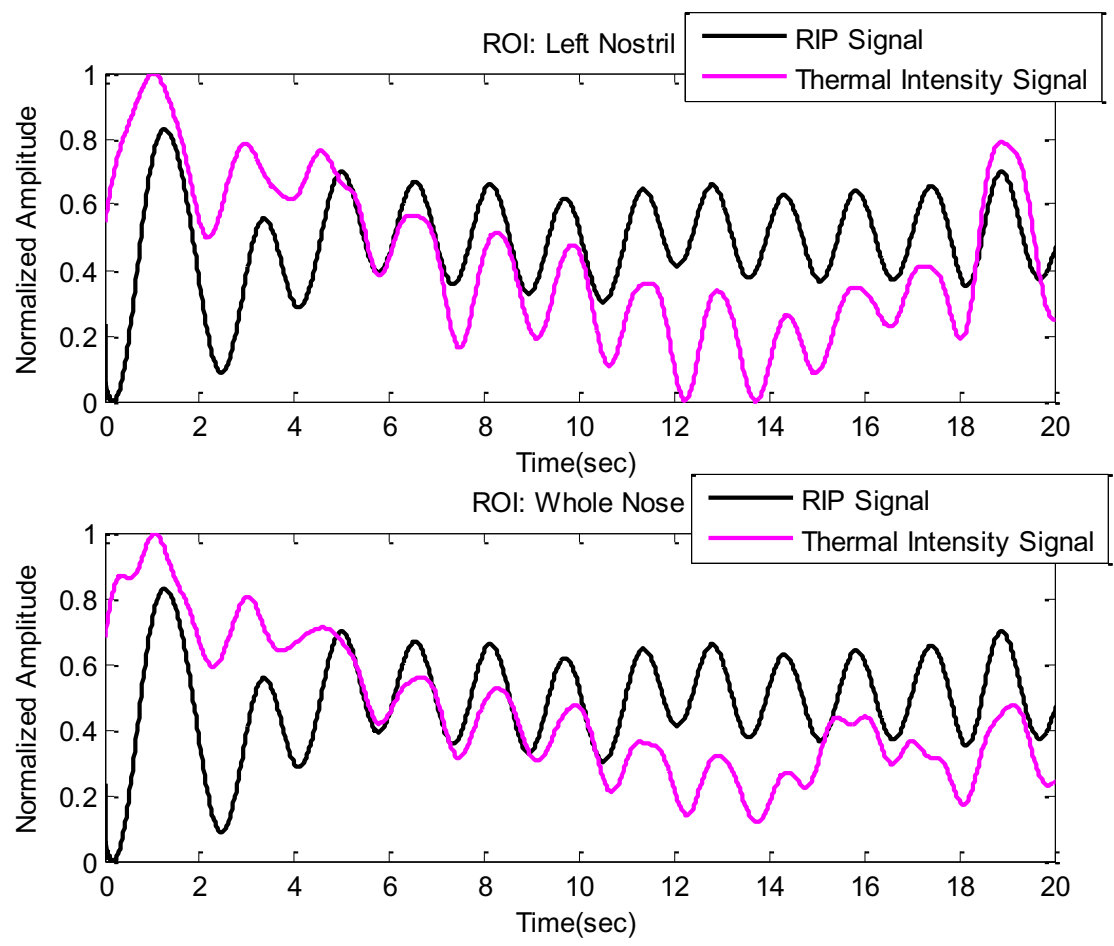

Figure 8.5. Hyperventilation intensity values plotted with RIP signals.

The results from the OSA trial, for which the subject exerted respiratory effort while closing the throat to cease air inhalation, are seen in Figure 8.6. This figure depicts the 
normalized (between 0 and 1) mean intensity of the tight left nostril ROI plotted with the RIP band signal. The RIP signal clearly depicts chest movement while the mean intensity signal flattens, indicating a lack of air flow.

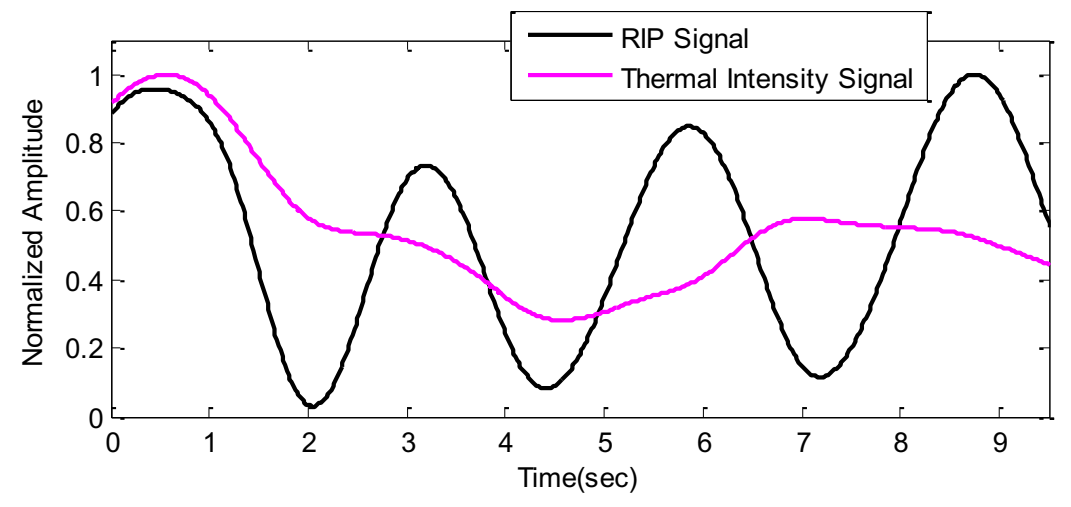

Figure 8.6. OSA simulation intensity values plotted with RIP signals.

Respiration signals were also extracted from an automatic ROI detection algorithm. This algorithm allows for more realistic conditions, in which subjects are sitting, but moving naturally through consecutive respiration tests. Each resulting thermal video was subjected to the facial recognition and region identification analyses described in Chapter 4. These methods optimized a number of parameters (subject dependent optimization) to successfully identify the region just under the nose for the majority of the thermal video. Method 1 resulted in an $80 \%$ accuracy while Method 2 resulted a 90\% accuracy and a larger signal amplitude. The extracted ROI mean intensity signals from each method are depicted in Figure 8.7 and Figure 8.8 between seconds 20 and 150; the time immediately following induced initiation noise, to the end of the video. The signals depict regular breathing for approximately 60 seconds, followed by the absence of breathing for an approximate 15 seconds, regular breathing again for 30 seconds, hyperventilation for 15 seconds and then OSA simulated breathing. When compared to the reference chest strap signal, Method 1 mean intensity signal mirrored regular 
breathing and the absence of breathing well, whereas hyperventilation and OSA simulated breathing were not well captured. Method 2 mean intensity signal captured regular breathing, the absence of breathing and hyperventilation, however OSA breathing was not well captured. Signal noise was an issue in extracting hyperventilation rates; the frequency at which the subject hyperventilated (around $1.5 \mathrm{~Hz}$ ) was high and introduced a lot of noise to the signal. Despite the noise, the Method 2 mean intensity signal extracted from the hyperventilation breathing accurately captured the quickened breathing rate. This is depicted in Figure 8.9. For both methods the OSA simulated breathing was depicted in the mean intensity signals as a small, downward change in the signal, followed by a sharp increase; in other words an abnormal but not drastic change. This is expected, as this respiratory test was intended to simulate chest motion in the absence of nasal air exchange. The mean intensity signal alone is not enough to characterize hyperventilation or OSA breathing. Each plotted signal was normalized and plotted at staggered baseline values.

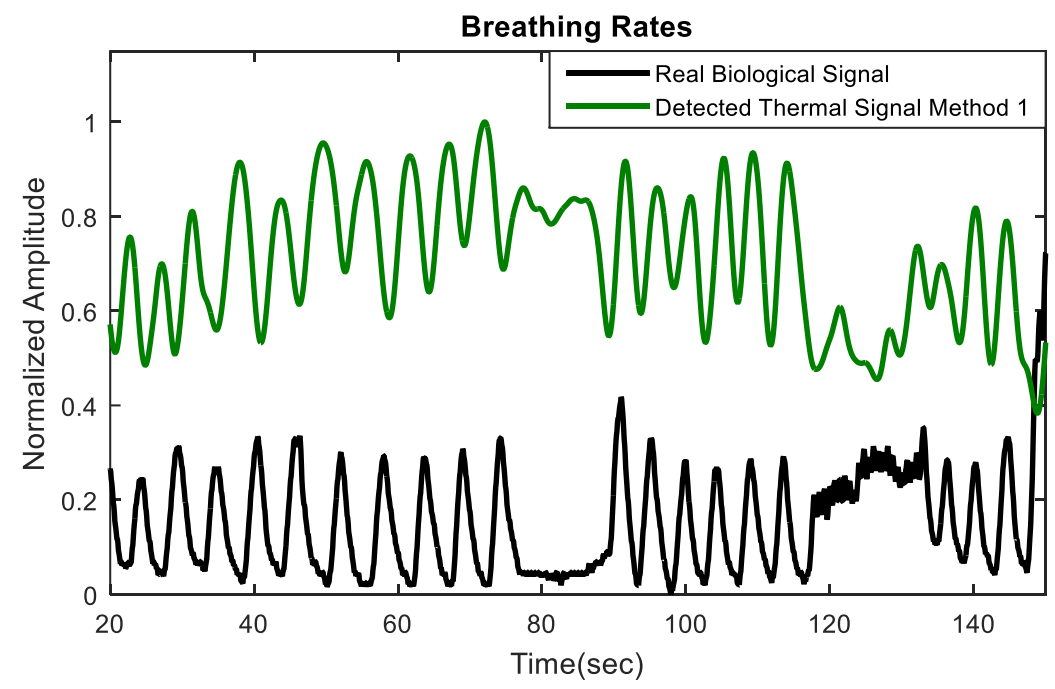

Figure 8.7. Breathing rate as detected by the chest strap (true biological signal) and the calculated Method 1 ROI mean intensity signal. 


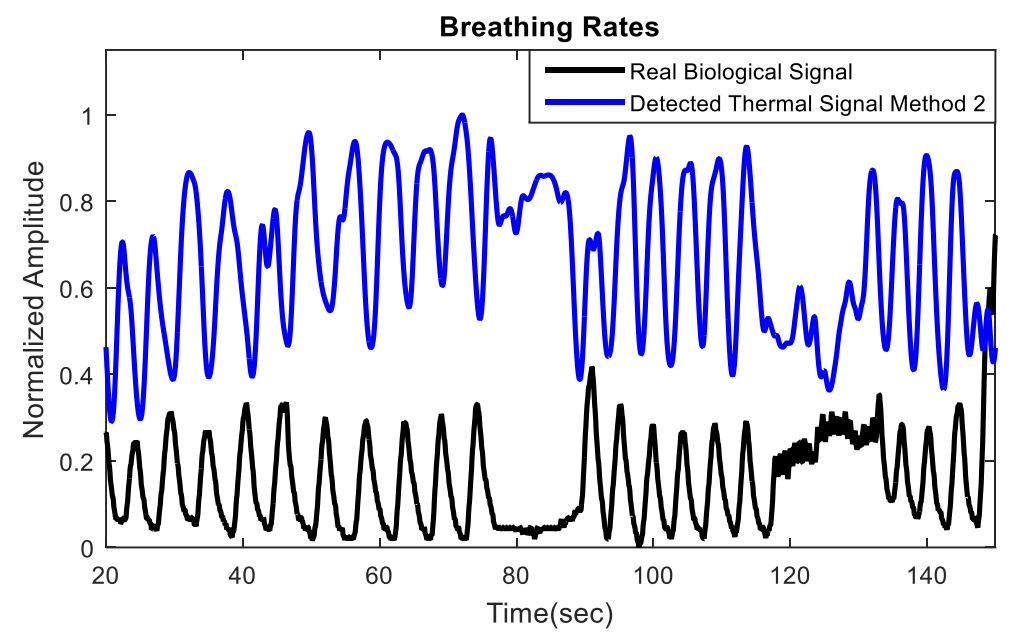

Figure 8.8. Breathing rate as detected by the chest strap (true biological signal) and the calculated Method 2 ROI mean intensity signal

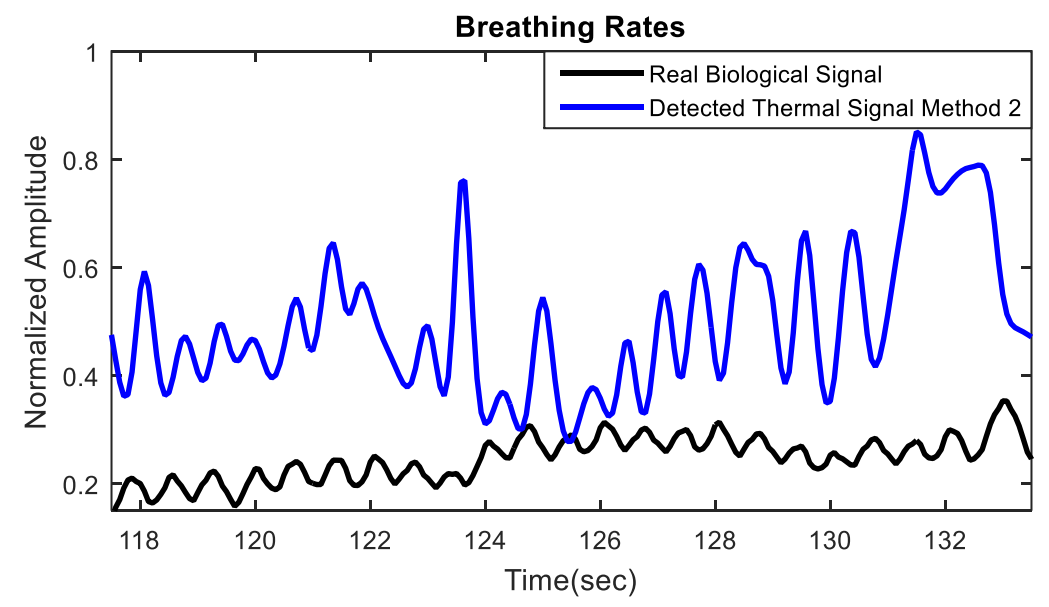

Figure 8.9. Breathing rate as detected by the chest strap (true biological signal) and a thermal-based measurement for the hyperventilation test.

The motion-based SAD signal was able to mirror the breathing tests well, as depicted in Figure 12. The SAD signal accurately depicts regular breathing and the absence of breathing. The signal then depicts the return to normal breathing and was able to accurately depict the hyperventilation test. This can be seen in Figure 8.10. The SAD signal then indicated a return to normal breathing and more aggressive movement associated with the OSA breathing. While 
the SAD signal was able to reflect all respiratory tests, including the hyperventilation and OSA simulated breathing, the signal amplitude varied in a manner not representative of physical movements. Furthermore, the amplitude diminishes over time, due to overall body movement, in addition to respiratory movement. In contrast, the mean intensity signals depicted an amplitude impervious to shifts in position and are representative of the amount of incoming and exiting airflow.

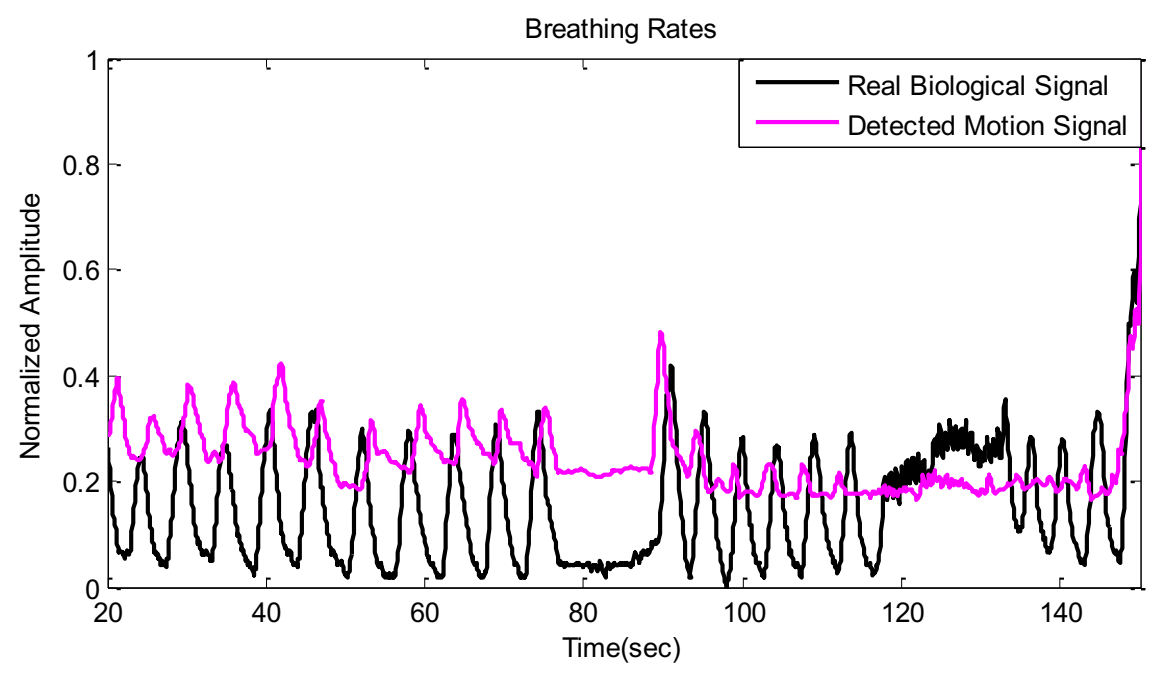

Figure 8.10. Breathing rate as detected by the chest strap (true biological signal) and the motion-based measurement. 


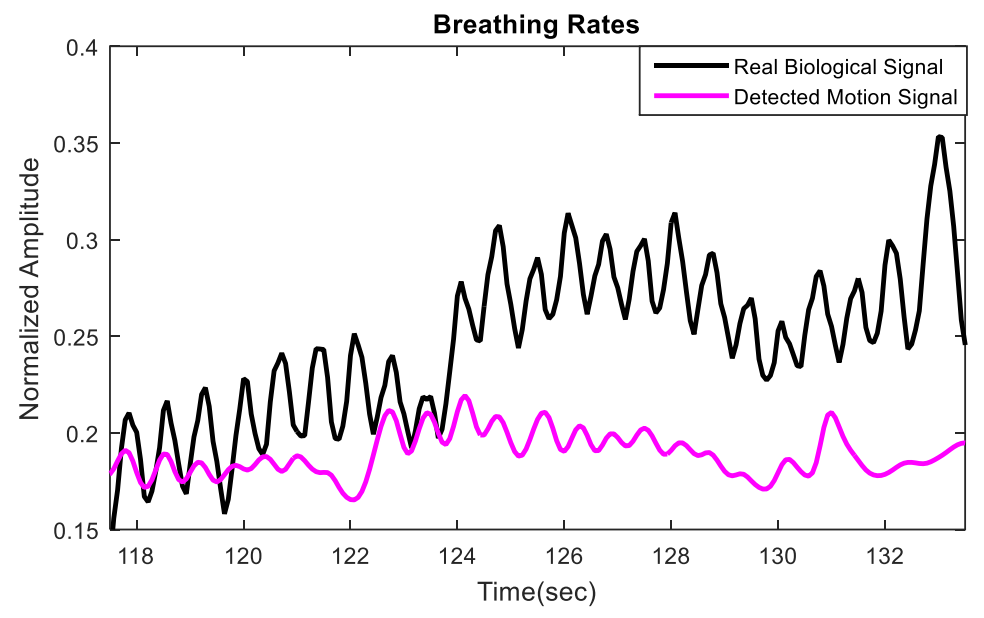

Figure 8.11. Breathing rate as detected by the chest strap (true biological signal) and the motion-based measurement for the hyperventilation test.

\subsection{Discussion}

The intensity based measurements and analyses successfully enhanced the thermal patterns of normal and abnormal nasal breathing. Thermal intensity signals and RIP breathing rates correlated very well; this was visually identifiable. Furthermore, the respiratory data resulting from the breathing trial simulating OSA clearly depicted chest movement (respiratory effort) while the corresponding mean intensity signal depicted flattened amplitude indicative of stagnant air flow. These are important findings as they demonstrate the potential of thermal cameras in the monitoring of the respiration vital sign, as well as in the detection of OSA; a notoriously difficult condition to identify and distinguish from CSA.

The motion based measurements with the facial recognition and region identification methods developed were able to follow the subject's movements with relative accuracy, however these methods can be improved for more robust accuracy and signal extraction. The reference frame could also be optimized; the signal amplitude diminishes over time due to overall body 
movement. The respiration signals extracted were capable of distinguishing between normal respiration, the absence of respiration and hyperventilation.

Considering the attributes and deficits of the motion based and intensity based signals, the information extracted is statistically independent and could be fused to optimize and improve signal reliability.

\subsection{Conclusion}

Thermal video data was subjected to intensity analysis with and without region tracking to encompass temperature changes over time, and sum of absolute differences analysis to encompass motion over time. The results suggest that temperature and motion methods are able to detect normal respiration, and that region tracking improves the accuracy of these measurements. However, one method itself is not sufficient to extract all relevant respiration information from thermal video for detection of OSA breathing; the temperature based processing better indicated the volume of airflow, whereas the motion based processing better detected absence of breath and OSA chest motion. These results suggest that the fusion of information from different physical phenomena could best detect respiration patterns, particularly OSA. Future work therefore involves the examination and comparison of other temperature-based and motion-based methods, in addition to facial recognition and tracking methods, followed by the examination of fusion methods. Additional future work includes the testing of fusion algorithms with many more subjects and the testing and compensation of significant noise, interference and motion. 


\section{Chapter 9}

\section{Temperature and Mobility Measurement Extraction}

This chapter builds upon the previous chapters by detailing the specific experimentation, data analysis, results and discussion associated with temperature and mobility.

\subsection{Introduction}

The entire algorithmic system is depicted in Figure 7.1 below, where this chapter focuses on the algorithmic processes and outcomes of temperature and mobility measurements, the component highlighted red. The following sections will detail specific image and signal processing methods used to estimate the vital signal measurements, and the effectiveness of the proposed methods in the presence of noise and signal abnormalities.

This chapter details each of three vital signal measurements in two separate sections, which refer to several image processing methods detailed in previous chapters. This includes image smoothing and enhancement, detailed in Chapter 4, Region of Interest identification, detailed in Chapter 5, and Adaptive Eulerian Video Magnification (AEVM), detailed in Chapter 6. 


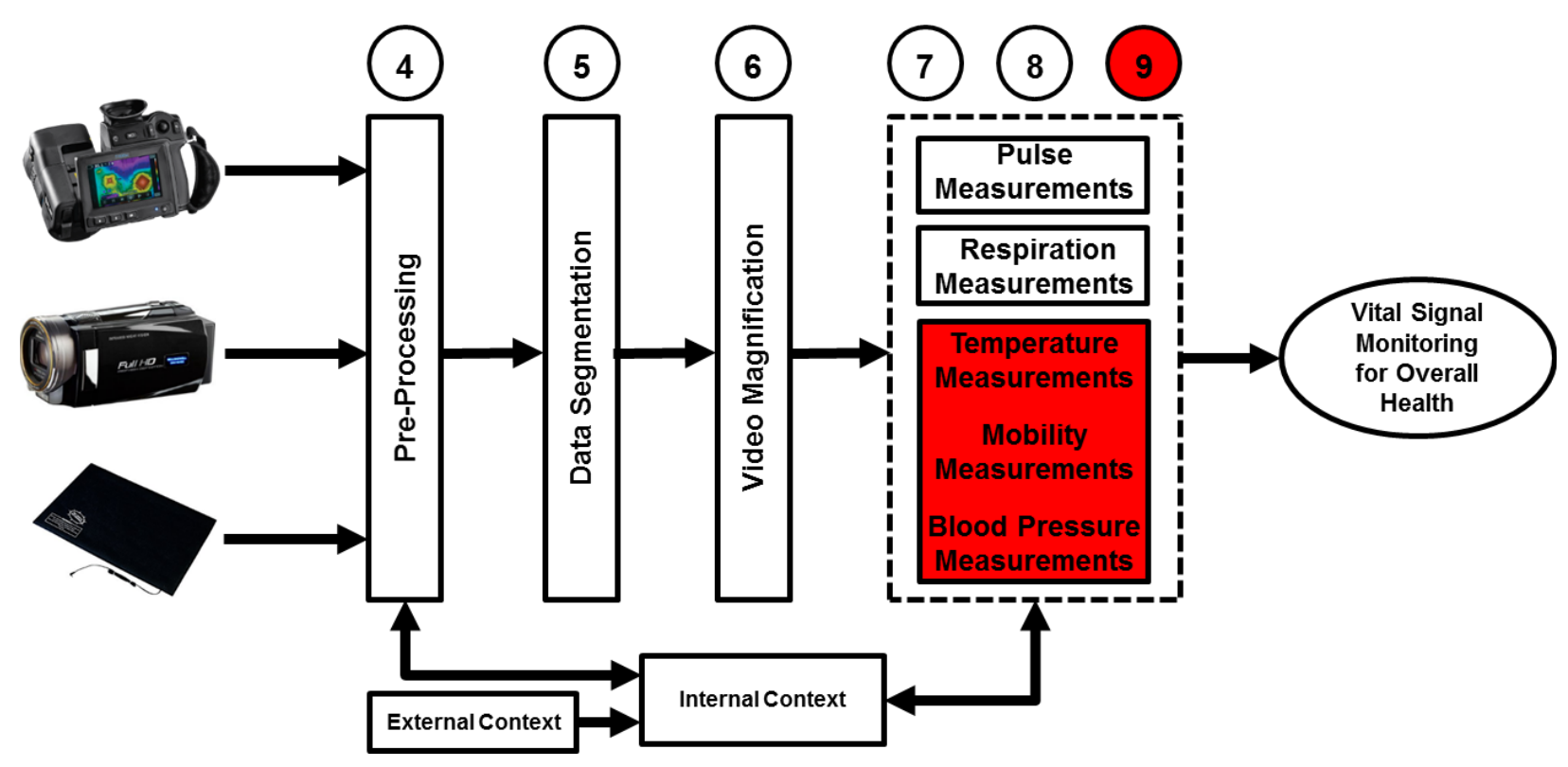

Figure 9.1 Algorithmic system with highlighted chapter focus

\subsection{Mobility Measurements}

Previous work (thesis) used several different mobility measurements to assess in-bed mobility of older adult patients and their respective health outcomes. This thesis further explores the potential of a particular measurement, Center Of Pressure (COP), in the examination and assessment of mobility and explores continuous in-bed motion data from healthy adults and an older adult patient, as opposed to discrete motion data from healthy adults examined previously. This chapter thesis focuses on mobility as it relates to temperature and pressure ulcer development. The following sub-sections briefly review previous chapters, then provide details on mobilty measurement extraction and discusses results.

\subsubsection{Equipment and Experimentation}

The equipment used in the experimentation specific to mobility measurements were inclusive of three pressure sensitive mats manufactured by S4 sensors (formerly Tactex Sensors) and 
associated software were used to gather pressure data from volunteers. Previous work (masters) used data gathered from 30 subjects in discrete sets. This thesis builds upon this work by examining more realistic, continuous data sets (as opposed to discrete data sets), further examining the effectiveness of one measurement; Center of Pressure (COP), and the extraction of mobility measurements from older adult patients.

One hospital bed was equipped with three pressure sensitive mats. Two young, healthy, female non-patient volunteers were recruited to perform a series of movements on the hospital bed that constituted four continuous data sets. Each subject began in a lying position, rolled to each side of the body, then transitioned from lying, to sitting, to a final standing position. These positions can be seen in Figure 9.2.

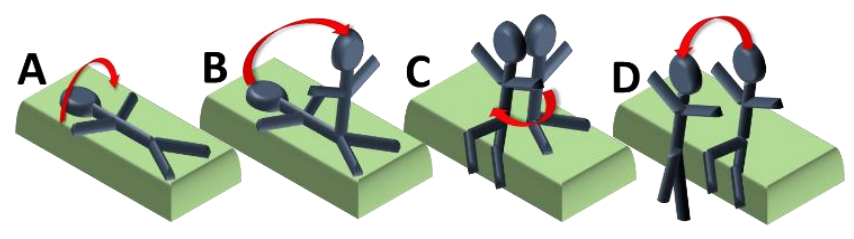

Figure 9.2 Mobility subject movement series

Additional mobility measurements were captured of patients susceptible to the development of pressure ulcers in a hospital setting. Experimentation consisted of capturing pressure data on a continuous basis. Pressure mats were placed underneath each of eight patient's mattresses and collected data 24 hours a day, 7 days a week. In addition to pressure data, thermal images were captured of patient's heels and malleoli once a week for several months. The timeline varied based on the individual patient, for example any given patient may be moved at any given time to a different hospital. Data was examined over one night for one 
patient to observe possible relationships between pressure and temperature distribution (presented also in the temperature measurement section).

\subsubsection{Mobility Measurement Data Analysis}

Healthy adult subject pressure data was first pre-processed; the weight of the mattress was accounted for. The data analyses described in this section aims to evaluate the potential of COP to gain insight on positional states and state transitions. This builds upon previous COP work by examining acceleration and velocity of the COP path as well as the COP [4].

The COP was calculated for each data frame as a Euclidean combination of the $\mathrm{x}$ and $\mathrm{y}$ COP signals. The velocity and acceleration signals were also calculated and are presented in this section. These features were calculated as per Equation 9.1 and Equation 9.2, where $y_{r, c}$

and $x_{r, c}$ are the $\mathrm{x}$ and $\mathrm{y}$ locations of respective sensors. Equation 9.3 describes the calculation of the total sum of sensor values at every point in time $(\operatorname{TS}(t))$, which is used in COP calculations. COP velocity and acceleration were calculated as in Equation 9.4 and Equation 9.5, where $\operatorname{COP}(t)$ represents a Euclidean combination of the $\mathrm{x}$ and $\mathrm{y} C O P$ signal at every point in time. The COP signals were observed for patterns related to positions and state transitions, the results for which are presented in the following section.

Equation 9.1

$$
\operatorname{LatCP}(t)=\frac{1}{T S(t)} \sum_{c=1}^{8} \sum_{r=1}^{9} y_{r, c} * \operatorname{sensor}_{r, c}(t)
$$

Equation 9.2

$$
\operatorname{LonCP}(t)=\frac{1}{T S(t)} \sum_{c=1}^{8} \sum_{r=1}^{9} x_{r, c} * \operatorname{sensor}_{r, c}(t)
$$


Equation 9.3

$$
T S(t)=\sum_{c=1}^{8} \sum_{r=1}^{9} \text { sensor }_{r, c}(t)
$$

Equation 9.4

$$
V_{C O P}(t)=(\operatorname{COP}(t)-\operatorname{COP}(t-1)) /(t-(t-1))
$$

Equation 9.5

$$
A_{C O P}(t)=(\operatorname{COP}(t)-\operatorname{COP}(t-1)) /(t-(t-1))^{2}
$$

The older adult patient pressure data resulting from one night of sleep (preceding thermal image collection the next morning) was then analyzed. This thesis examines in-bed mobility as it related to temperature and pressure ulcer development in the heels, and therefore considers only the pressure data resulting from the mat situated under the patient's feet. The night was considered inclusive of the time frame between 9pm and 8am; hours chosen based on hospital observation and staff schedules. All pressure data were subject to the following analyses.

Raw data was first calibrated. An hour in the day during which the patient was out of bed was first identified. The average sensor output over this hour was calculated for each individual sensor, resulting in an average reading of the mattress weight. Average mattress sensor scores were subtracted from respective sensor outputs for all subsequent pressure data.

To gain a general understanding of the pressure patterns exerted by the patient's feet during the night, the average exerted pressure was calculated. The patient under examination was known to be in and out of bed often throughout the night. To get a representative average, times at which the patient was in bed were first identified by using a threshold. This threshold was chosen based on observation and examination of data. Data at these points in time were 
gathered, averaged and depicted as an average pressure image. The average pressure exerted by the left foot and the average pressure exerted by the right foot were compared.

To gain a general understanding of the level of movement experienced by each foot during the night, the standard deviation of exerted pressure was found. The more a patient moves, the more sensor outputs vary; therefore, the standard deviation of exerted pressure represents the level of movement. Sensor data was gathered at times during which the patient was in bed, and the standard deviation of these outputs were calculated. The level of movement was identified in the left foot and compared to the right foot.

\subsubsection{Results}

The continuous data sets for both healthy adult subjects were subjected to the described analyses. The COP in the $\mathrm{x}$ and $\mathrm{y}$ directions as well as the COP velocity and acceleration can be seen in Figure 9.3 for the one continuous data set. Figure 9.4 depicts three example discrete COP data sets. The pink plots depict the $\mathrm{x}$ and $\mathrm{y}$ COP and COP velocity and acceleration for a subject performing a lie-to-lie transition. The black and green plots of Figure 9.4 depict the same signals for a lie-to-sit transition and a sit to stand transition, respectively. Three circles appear on Figure 9.3; a pink circle that highlights the time during which the subject was performing a lie-to-lie scenario, a black circle that highlights the time during which the subject was performing a lie-to-sit scenario, and a green circle that highlights the time during which the subject was performing a sit-to-stand scenario. All signals in the plots in Figure 9.4 correspond strongly to the respective circled movements in Figure 9.4. 


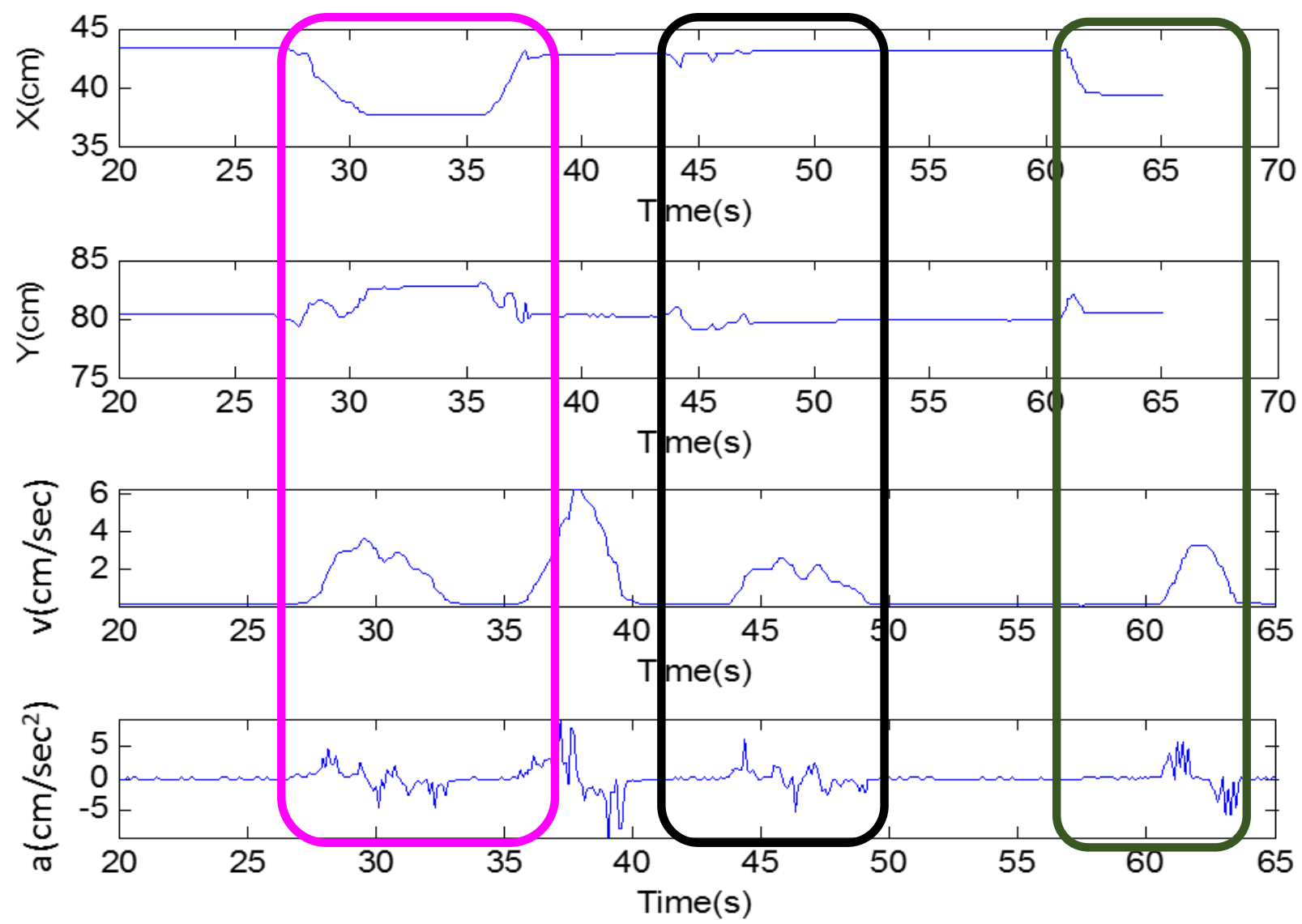

Figure 9.3 COP signals for a lie-to-sit-to-stand movement.
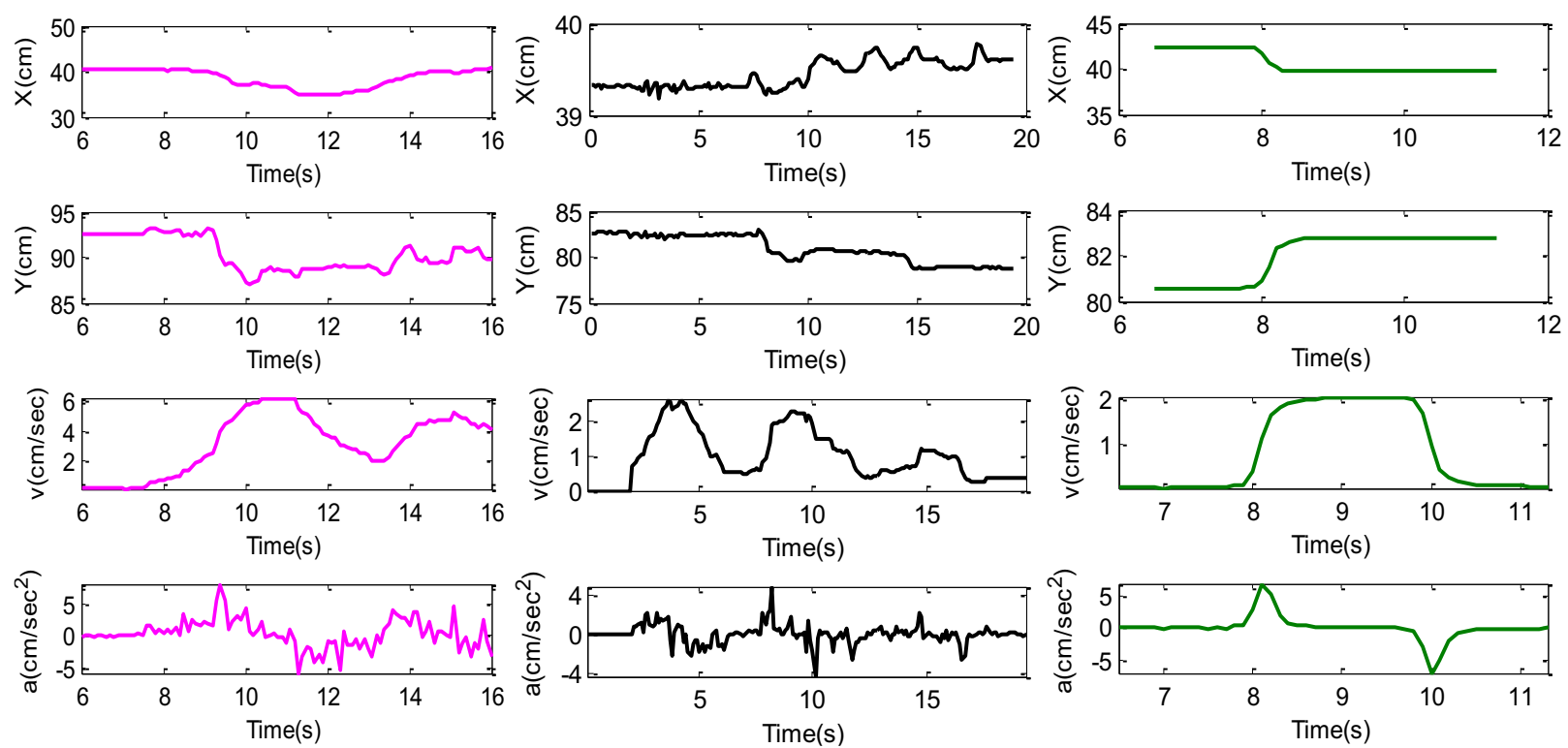

Figure 9.4 COP signals for lie-to-lie, lie-to-sit and sit-to-stand transitions. 
Patient pressure data were analyzed through statistical measurements to understand general sleeping behaviors during one night. Average exerted pressure was found by averaging sensor scores temporally across identified periods during which the patient was in bed. A representation of movement by the heels was obtained by calculating the standard deviation of sensor scores across the same time periods. It is visually clear in Figure 9.5, that the average pressure exerted by the left heel was higher than the pressure exerted by the right heel. It can also be seen that the amount of movement the left foot experienced was lower than the amount of movement the right foot experienced. In other words, the left heel was subjected to a higher amount of pressure with less relief due to movement when compared to the right foot, which was subjected to a lower amount of pressure and relieved often due to a higher amount of movement. Ratio calculations ( $\mathrm{L} / \mathrm{R})$ confirmed these observations; the ratio of average pressure exerted by the left heel to the right heel was approximately 1.52 , whereas the ratio of left heel standard deviation (level of movement) to right heel was approximately 0.40 .

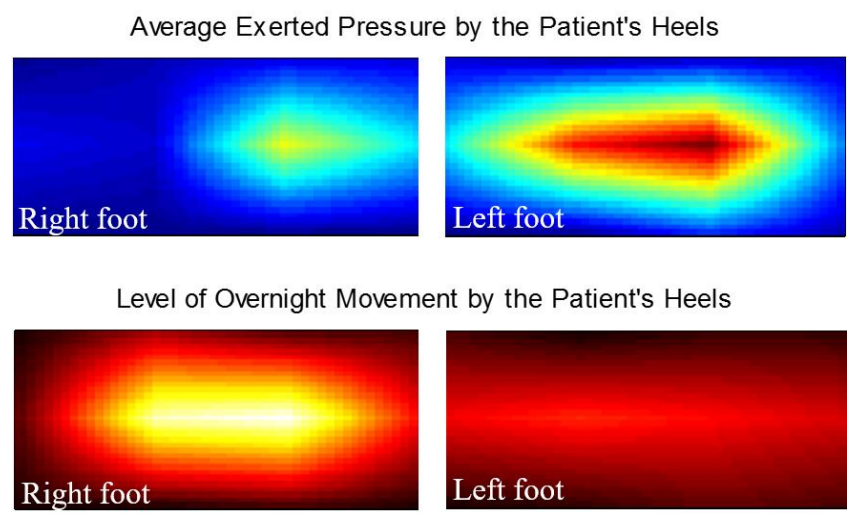

Figure 9.5. Time averaged, filtered sensor values and standard deviations over the duration of one night.

The patient's feet were also examined via thermal camera; the details for which can be found in Section 9.3, as well as the discussion surrounding the possible relationship between the mobility measurement results and the thermal measurement results. 


\subsubsection{Discussion}

The COP mobility measures (COP, COP acceleration and velocity), extracted from healthy, adult subjects were independently able to characterize the subject's state transitions. These results suggest that COP measures can further inform algorithmic systems assessing a patient's positional states and state transitions.

The average exerted pressure and movement measurements, extracted from one older adult in-bed patient, were able to indicate a general overnight pressure pattern of the feet; the left heel was subjected to a higher amount of pressure with less relief due to movement while the right foot was subjected to a lower amount of pressure and relieved often due to a higher amount of movement. In theory, less pressure relief would mean less blood flow and an increased chance of pressure ulcer development. Corresponding temperature measurements, which can be an indication of blood flow, are examined in Section 9.3 with reference to the mobility measurement results presented here.

The work completed in this section aimed to contribute to the automated analysis of in-bed patient health by extracting mobility measurements from pressure data. Previously, pressure mat data has been used to assess the ability of an in-bed subject to move independently. This includes automatic detection of subject movement, or the inability to move; the referenced algorithmic system was able to detect subject pressure points, the relief of pressure points, and the length of time for which pressure points remained under pressure. This thesis builds upon previous work by further examining the potential of mobility measurements, in conjunction with temperature measurements in the observation, detection and prevention of pressure ulcers. This section examined real time, continuous patient data gathered from healthy adults and in- 
bed patients. While only pressure data is examined in this section, the next section discusses corresponding temperature measurement results and the possible relationship between mobility measurements, temperature measurements and the development of pressure ulcers.

\subsubsection{Conclusion}

Previous work has been able to distinguish between different in-bed patient orientations. This thesis has demonstrated the potential for COP to be used in identification of several positional transitions. One important in-bed mobility related health outcome is pressure ulcer development, which is related to blood flow, and as a result skin temperature. In this sense mobility and temperature are closely related, and the results of the mobility and temperature measurement analysis are complementary.

Identification and distinction of and between different in-bed movements is a first step towards the automation of in-bed mobility tools. This section built upon a previous algorithmic system capable of identifying and distinguishing between three different positional states and three state transfers. This section was able to shed light on some of the complexities of these states and state transfers through several different methods including pressure point and COP

monitoring. With further development, an algorithmic system could be implemented into a hospital setting to assist with, and eventually replace human identification and assessment of elderly patient movement; a much needed hospital service for an expanding elderly population.

\subsection{Temperature Measurements}

This thesis examines body temperature in human subjects; namely the relationship between temperature and pressure, how temperature can inform blood flow and perfusion, and the development of pressure ulcers. Data from healthy adult volunteers performing controlled 
experiments was first examined with the goal of identifying and quantifying thermal changes occurring in response to applied pressure. Temperature measurements of patients susceptible to the development of pressure ulcers in a hospital setting were then examined, to identify and quantify thermal changes in response to applied pressure in at-risk subjects. Long term thermal data was then examined for one 92-year-old female, who began development of a pressure ulcer over a duration of 112 days. The following sub-sections breifly review previous chapters, then provides details on temperature measurement extraction from pre-processed, region specified thermal image data, and discusses results.

\subsubsection{Equipment and Experimentation}

The equipment used in the experimentation specific to temperature measurements were inclusive of a thermal camera, a pressure sensitive mat, a laptop and a Wavetex Meterman thermometer. Technical specifications for all equipment are detailed in Chapter 4.

A FLIR A-series IR camera was used to gather thermal data from one healthy subject applying pressure to their hands, and from two older adult patient's feet. Subjects were either seated with their feet on the floor, or lying in bed while thermal images of their feet or hands were captured. For the healthy adult subject experimentation, the pressure sensitive mat was placed on the floor and used to distinguish between two general levels of pressure exerted (light pressure verses heavy pressure). The laptop was used to gather data from the pressure mat via Bluetooth as well as to save uploaded thermal images. The Wavetex Meterman thermometer was used to measure ambient temperatures (for validation purposes). All experimentation is detailed fully in Chapter 4. 


\subsubsection{Temperature Measurement Data Analysis}

For each stage of temperature measurement examination (experimentation with healthy subjects and in-hospital monitoring of at-risk older adult patients), thermal images were first subjected to pre-processing as described in Chapter 4 and region identification as described in Chapter 5.

This thesis first aimed to characterize temperature changes in response to a pressure applied in a healthy adult subject in a controlled environment. The region measurements extracted in Chapter 5 were the areas of respective temperature grades in standardized contoured thermal images (an example of which is depicted in Figure 5.22), and the maximum, minimum and mean intensity as well as the weighted thermal centroid coordinates of respective regions depicted in Figure 9.7. Each of the region measurements extracted are examined and analyzed over the entire recovery period of 10 minutes. The results are discussed in the following sections.

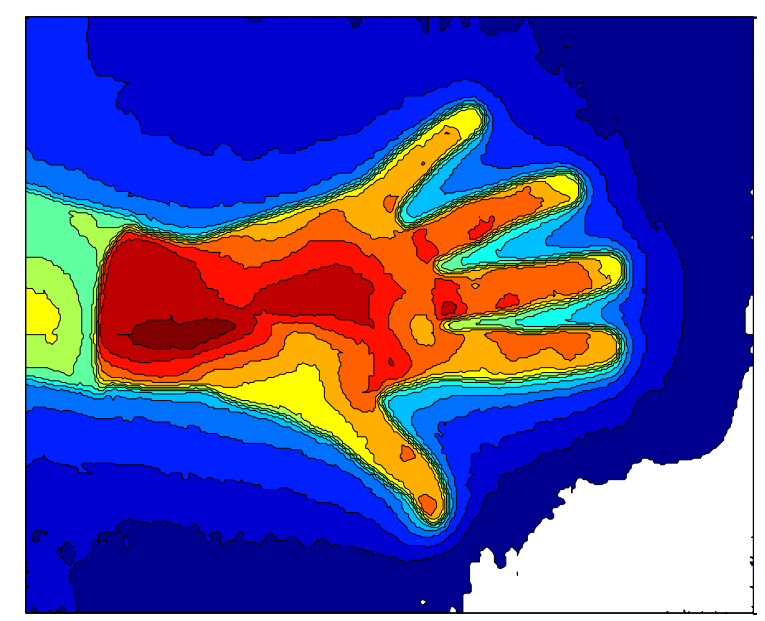

Figure 9.6. Contour plot at specified levels. 


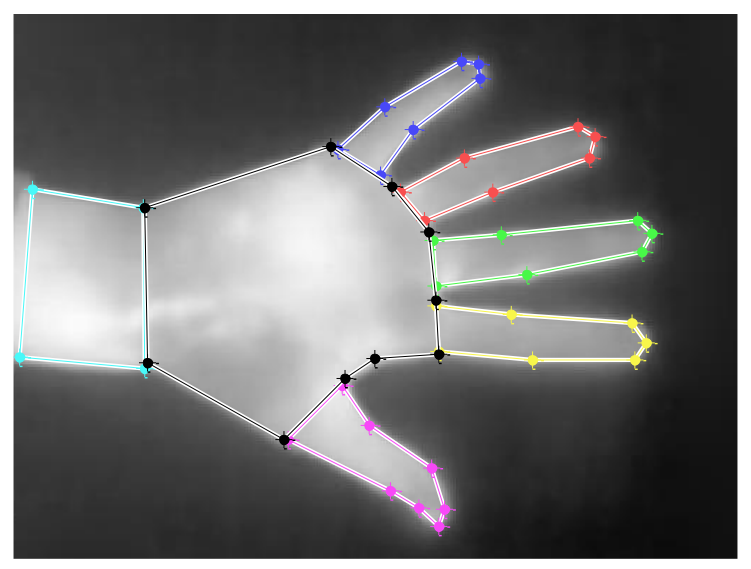

Figure 9.7. Depiction of the ROIs

The thesis then aimed to examine thermal patterns arising from at-risk older adult subjects who, on average, spend most of their time in bed. This thesis is concerned with pressure ulcers on the heels, and so patient heels were areas imaged. These images were analyzed through area contours to determine the temperature distributions for each heel. Area ratios of the left heel compared to the right heel were calculated as L/R. These measures of comparison were considered in addition to absolute measures to best discount the effects of external stimuli. The results are presented and discussed in the next sections, particularly as they relate to pressure mat data gathered from the same patient the preceding night of sleep.

In the examination of at-risk older adult in-bed patient's heels over time, data was extracted from one 92 year old female patient once per week over the entire duration of her hospital stay (with the exception of one month during which the subject moved locations). This resulted in 12 days of data collection over 116 days. Each of 4 thermal images captured per day (left and right heels, and left and right lateral malleoli) were subjected to region identification (as described in Chapter 5) and feature extraction. The mean intensity feature was calculated for each image set, resulting in 12 data points from each ROI (left and right heels and lateral malleoli). To account for changes in atmosphere and other external influences, the left heel was 
compared to the right heel. This assumes that on any given day, both feet were subjected to the same external influences. For each data collection day, and each anatomical landmark (heel, malleoli) the difference between the left ROI and the right ROI were calculated and examined. The results are presented and discussed in the following sections.

\subsubsection{Results}

The first measurement of thermal change in thermal images of healthy adult subjects was the area covered by each of 15 temperature levels. These areas represent the number of pixels at every $1.0^{\circ} \mathrm{C}$ temperature increment between $20.0^{\circ} \mathrm{C}$ to $35.0^{\circ} \mathrm{C}$. Figure 9.8 depicts the change in area (number of pixels) at each temperature level resulting from light applied pressure. The colors of the graph in Figure 9.8 correspond to the colors of respective temperature levels between $28.0^{\circ} \mathrm{C}$ to $34.0^{\circ} \mathrm{C}$ in contour plots seen in Figure 5.22. This specific temperature range was chosen to examine the subject's hand alone (excluding the background). It can be observed that the hand appeared to generally increase in temperature; the areas enclosing temperatures $32.0^{\circ} \mathrm{C}, 33.0^{\circ} \mathrm{C}$ and $34.0^{\circ} \mathrm{C}$ all increased over the ten minute period, whereas the other, lower temperature areas decreased over the ten minute period. This result correlates with a visual observation of the thermal images over time. Figure 9.9 depicts the same graph, but due to the application of heavy pressure. The same patterns are seen to occur, with all area trajectories reaching higher temperatures (than from the application of light pressure) by the end of the ten minute recovery period. This indicates that the application of heavy pressure resulted in a hotter hand than from the application of light pressure. 


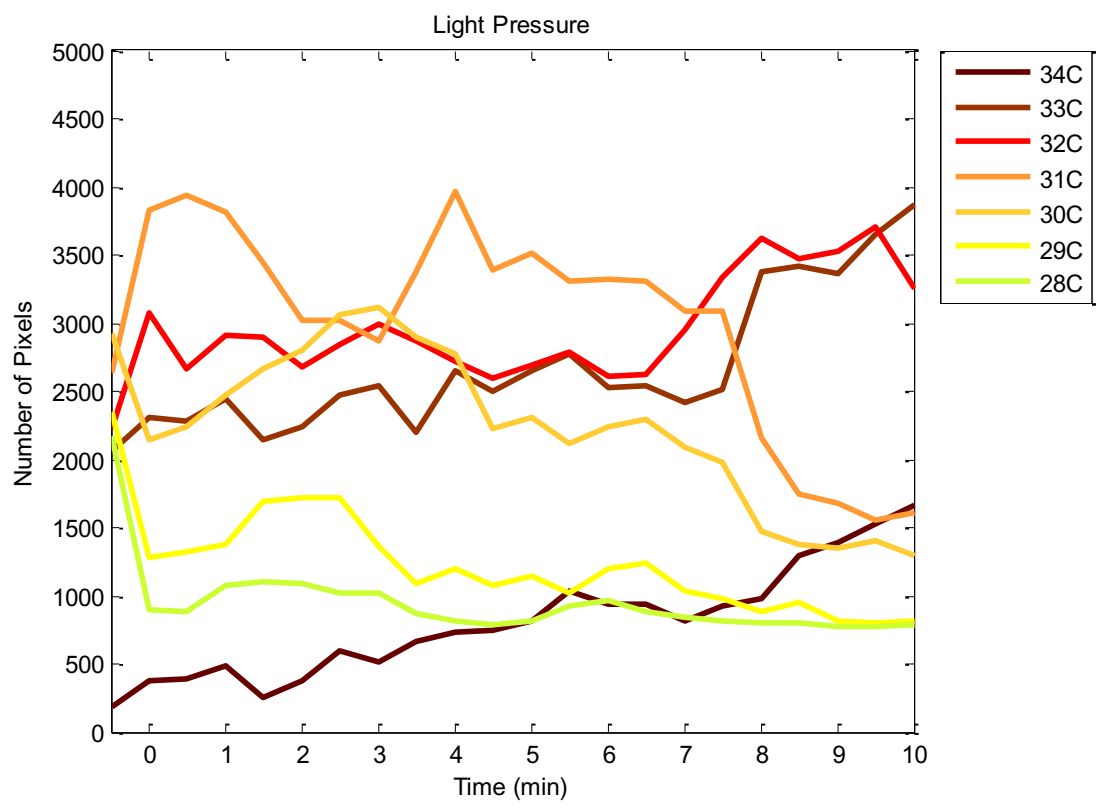

Figure 9.8 Changes in temperature areas due to light pressure over time.

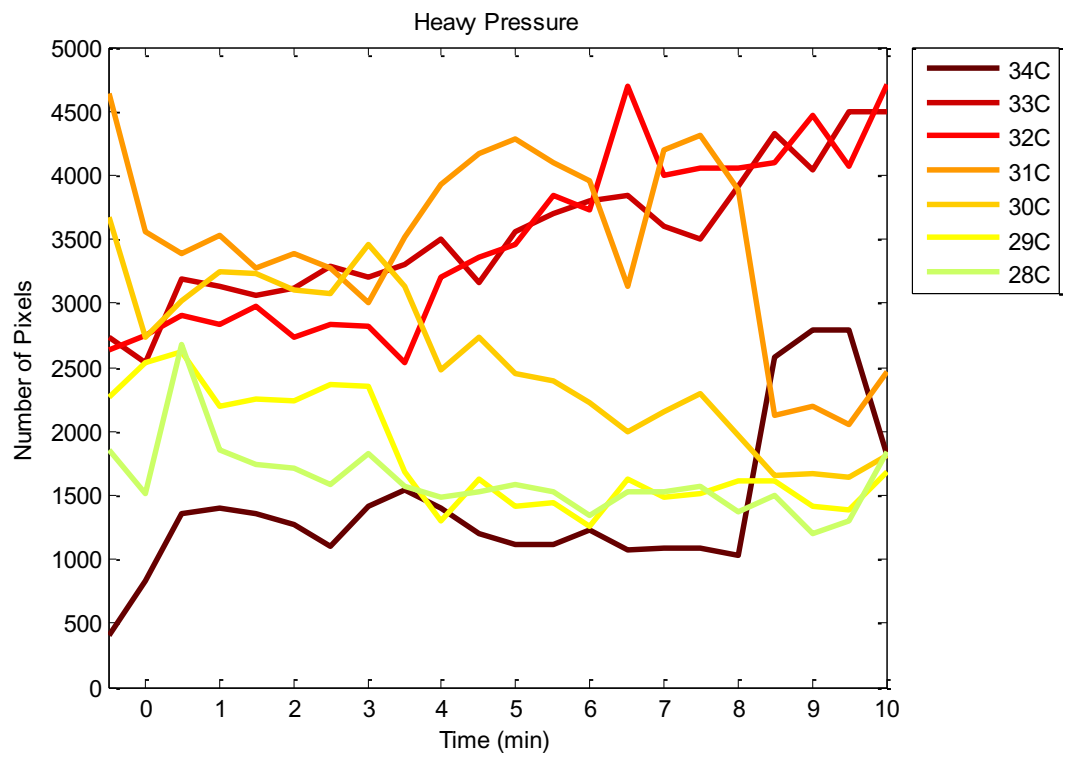

Figure 9.9 Changes in temperature areas due to heavy pressure over time.

The next set of measurements were the mean, minimum, maximum intensities and the location of the weighted intensity centroids. These were calculated within each ROI, for a total of 28 measurements from each image. All measures of intensity depicted the thermal patterns identified visually from contour plots. The mean intensity plots (capturing both the maximum 
and minimum measurements) can be seen in Figure 9.10 for the application of light pressure and Figure 9.11 for the application of heavy pressure. The fingers all become initially colder, then warmer over time to a temperature exceeding that of the baseline temperature. On average the palm and wrist increase in temperature over time to a much lesser degree, without the initial cooling. These ROI specific patterns are seen to occur again as a result of the application of heavy pressure.

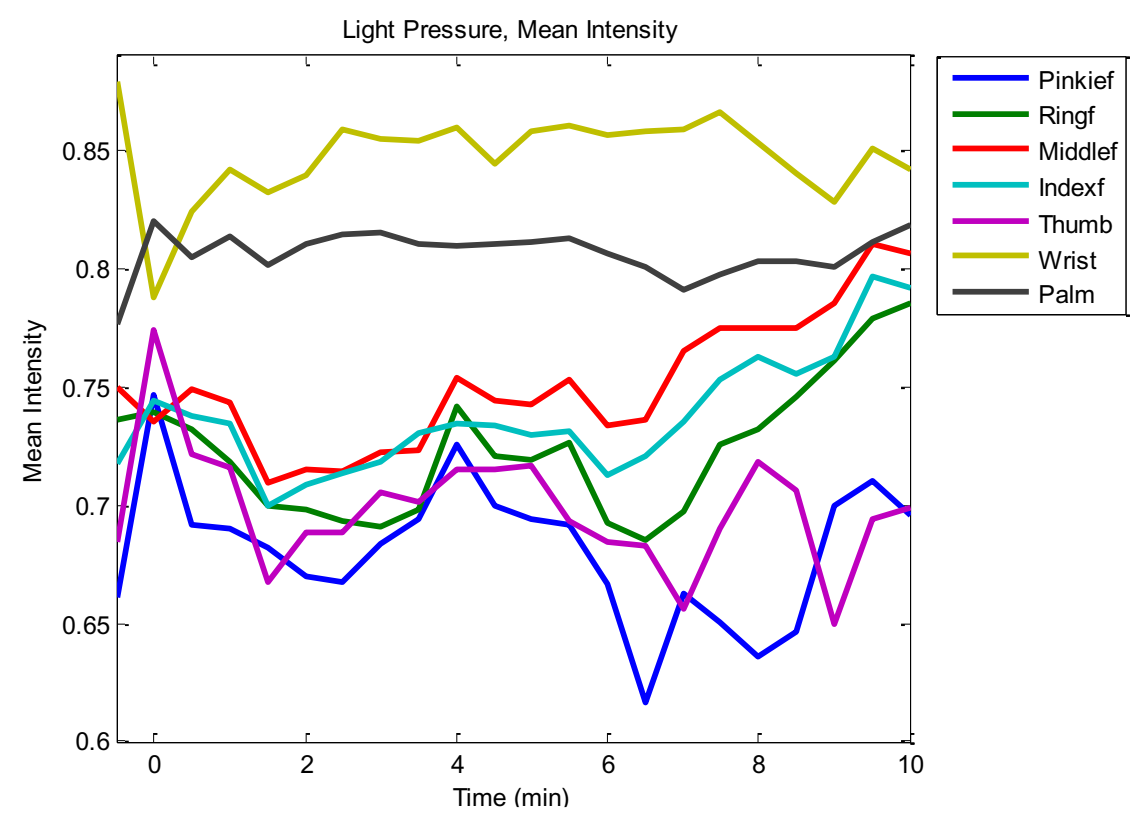

Figure 9.10 ROI specific mean intensities due to light pressure over time. 


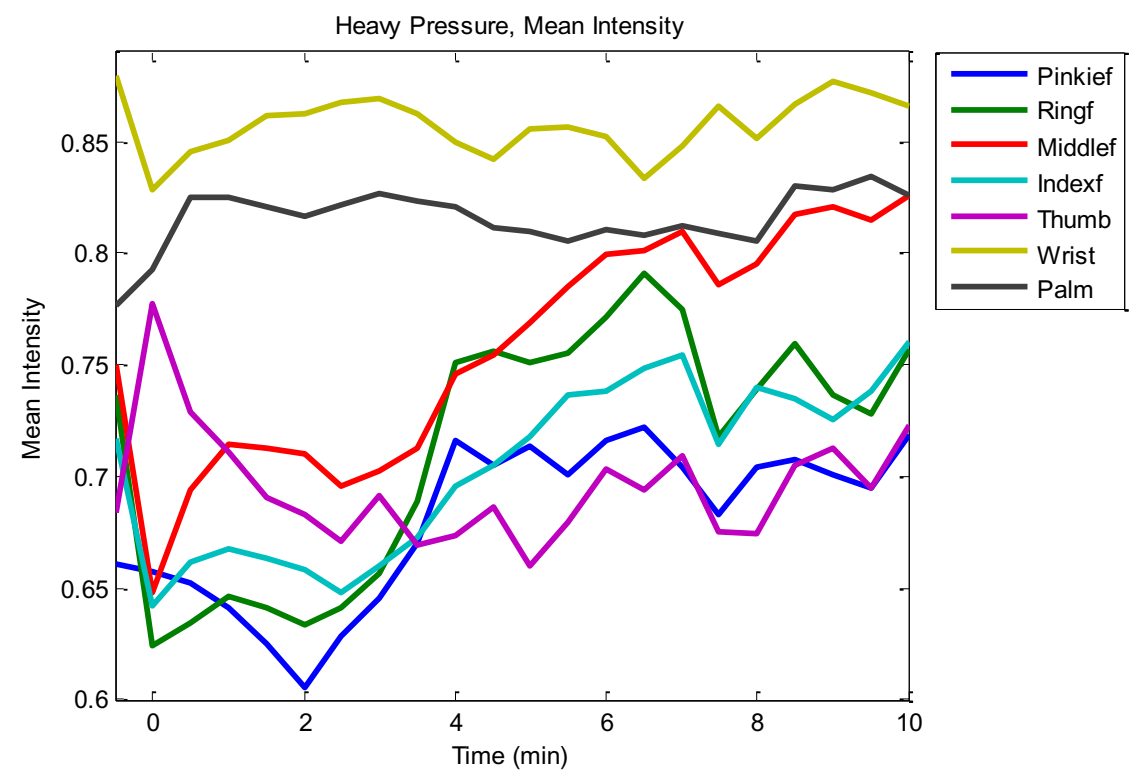

Figure 9.11 ROI specific mean intensities due to heavy pressure over time.

The intensity weighted centroids changed very little over the ten minute pressure recovery period. While the absolute temperature of the hand varied frequently, the temperature distribution did not change. This is depicted in Figure 9.12 and Figure 9.13, where it can be seen that each ROI centroid trajectory moves very little over the ten minute recovery period.

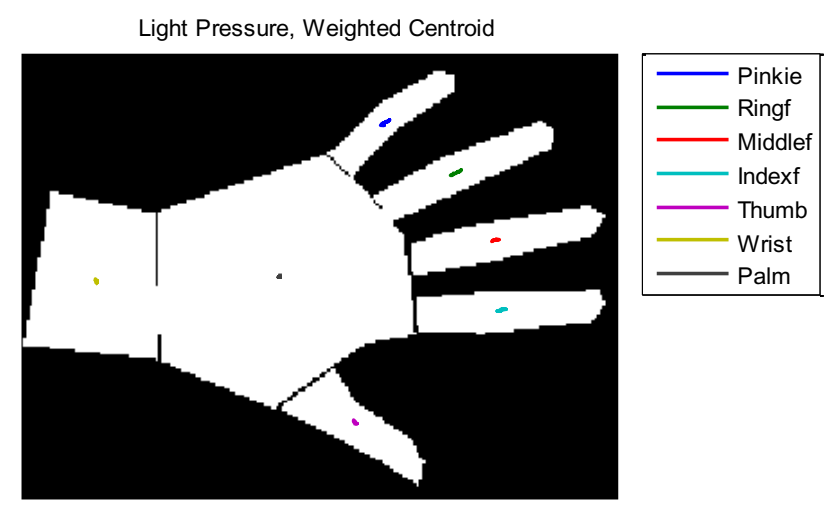

Figure 9.12 ROI specific weighted centroids due to light pressure over time. 


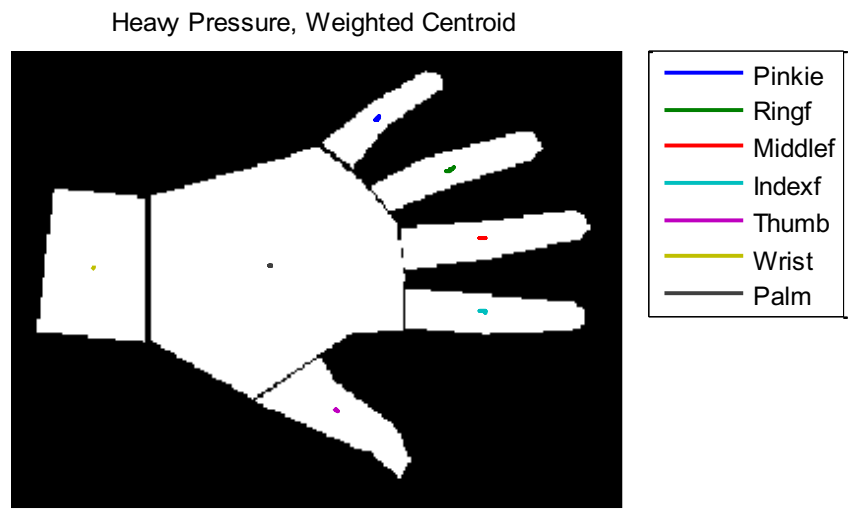

Figure 9.13 ROI specific weighted centriods due to heavy pressure over time.

The overall thermal pattern in a hand because of applied pressure was characterized by contour measurements while ROI patterns captured region-specific thermal patterns. The overall thermal pattern was consistent for both the light and heavy application of pressure; an initial cooling of the fingers followed by heating over time, while the wrist and palm (on average) heated more slowly over time without initial cooling. While the absolute temperatures of the hand varied, the distribution of heat (represented by a weighted centroid in each ROI) barely changed over the ten minute time period; a result that was not anticipated. The aggregate results of this study suggest that measurement extraction can confirm and quantify visually observed thermal behaviour and additionally, capture behaviour that was not anticipated and not visually identifiable.

Thermal images of at-risk older adult patients were analyzed through measurements of comparison in order to make thermal observations while minimizing consideration of external thermal influences. The thermal images were transformed into heat maps by calculating contours representative of each $1{ }^{\circ} \mathrm{C}$ between the temperature range of $23^{\circ} \mathrm{C}$ to $32^{\circ} \mathrm{C}$, as well as the area of skin (measured by number of pixels) at these specified temperatures. The thermal image contour plots of both the patient's feet and a healthy adult's feet can be seen in figures 
3 and 4, respectively. There is a large, visible difference in patient heel temperatures; the lower left heel is a cooler temperature when compared to the right heel. Contrary to patient results, the healthy adult heel temperatures are visibly similar. The ratios of areas at each temperature level for both patient and healthy heels is reported in Table 9.1, and confirmed visually identifiable patterns. The patient's left heel was colder than the right and $\mathrm{L} / \mathrm{R}$ ratios varied (in the patient, heel-specific temperature range of $26^{\circ} \mathrm{C}$ to $29^{\circ} \mathrm{C}$ ) from 0.29 to 3.67 . This is a wide range when compared to the healthy heel ratios (in the healthy, heel-specific temperature range of $24^{\circ} \mathrm{C}$ to $30^{\circ} \mathrm{C}$ ) varying from 0.77 to 1.84 . The table presents some outlier results when reporting area ratios at the two highest temperatures $\left(31^{\circ} \mathrm{C}\right.$ and $\left.32^{\circ} \mathrm{C}\right)$. This is because the left heel had area measured at these temperatures while the right heel had little to no area measured at these temperatures; the $\mathrm{L} / \mathrm{R}$ ratio is therefore very large.

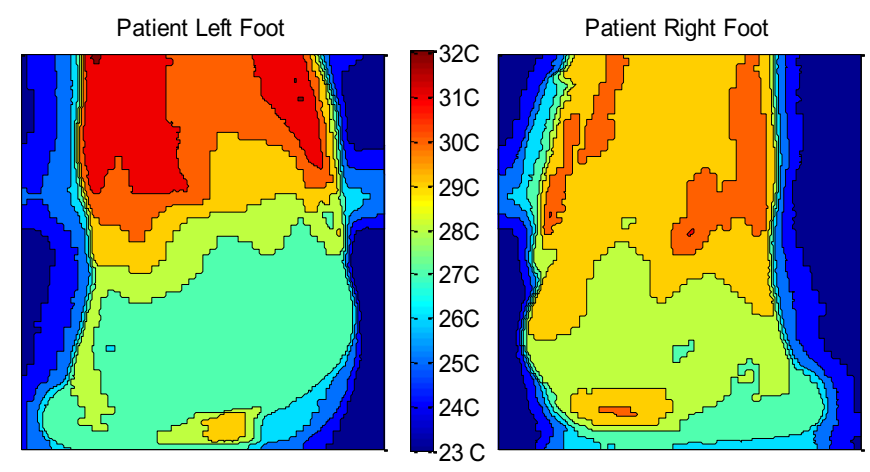

Figure 9.14 Contour plots of the patient heels. 


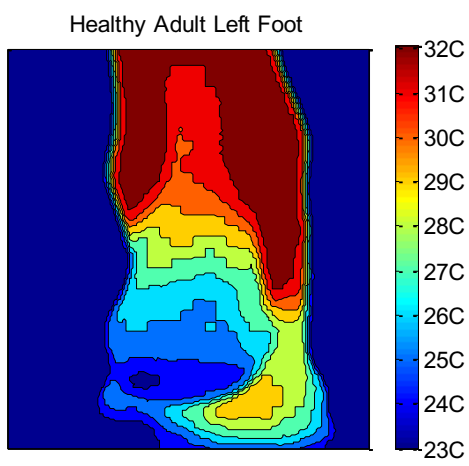

Healthy Adult Right Foot

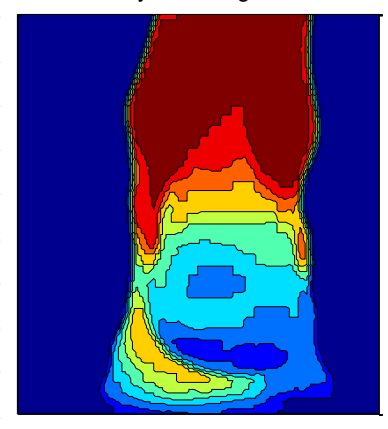

Figure 9.15 Contour plots of the patient heels.

Table 9.1 Area ratios at each temperature level for both patient and healthy adult heels

\begin{tabular}{|c|c|c|c|c|c|c|}
\hline \multirow{2}{*}{$\begin{array}{c}\text { Temp } \\
\left({ }^{\circ} \mathbf{C}\right)\end{array}$} & \multicolumn{2}{|c|}{ Patient Pixel Areas } & \multicolumn{3}{c|}{ Healthy Adult Pixel } \\
\cline { 2 - 7 } & $\begin{array}{c}\text { Left } \\
\text { Heel }\end{array}$ & $\begin{array}{c}\text { Right } \\
\text { Heel }\end{array}$ & $\begin{array}{c}\text { Ratio } \\
\text { L/R }\end{array}$ & $\begin{array}{c}\text { Left } \\
\text { Heel }\end{array}$ & $\begin{array}{c}\text { Right } \\
\text { Heel }\end{array}$ & $\begin{array}{c}\text { Ratio } \\
\text { L/R }\end{array}$ \\
\hline 23 & 5401 & 9268 & $\mathbf{0 . 5 8}$ & 9018 & 9315 & $\mathbf{0 . 9 7}$ \\
\hline 24 & 3535 & 3339 & $\mathbf{1 . 0 6}$ & 756 & 410 & $\mathbf{1 . 8 4}$ \\
\hline 25 & 4727 & 2914 & $\mathbf{1 . 4 6}$ & 1412 & 1685 & $\mathbf{0 . 8 4}$ \\
\hline 26 & 1918 & 2108 & $\mathbf{0 . 9 1}$ & 1149 & 1497 & $\mathbf{0 . 7 7}$ \\
\hline 27 & 15456 & 4206 & $\mathbf{3 . 6 7}$ & 1001 & 981 & $\mathbf{1 . 0 2}$ \\
\hline 28 & 5038 & 10272 & $\mathbf{0 . 4 9}$ & 934 & 608 & $\mathbf{1 . 5 4}$ \\
\hline 29 & 4477 & 15532 & $\mathbf{0 . 2 9}$ & 761 & 749 & $\mathbf{1 . 0 2}$ \\
\hline 30 & 5733 & 4346 & $\mathbf{1 . 3 2}$ & 567 & 453 & $\mathbf{1 . 2 5}$ \\
\hline 31 & 5471 & 15 & $\mathbf{3 6 4 . 7}$ & 1110 & 706 & $\mathbf{1 . 5 7}$ \\
\hline 32 & 244 & 0 & $\mathbf{I n f}$ & 2922 & 3226 & $\mathbf{0 . 9 1}$ \\
\hline
\end{tabular}

Thermal images of an in-hospital at-risk older adult were then examined to determine long term thermal patterns associated with pressure, and the consequent effects on perfusion and lack of blood flow. These images were subjected to image processing, region selection and feature extraction to observe pressure related temperature patterns over the duration of the patient's hospital stay. Following pre-processing and region identification, the mean intensity feature was extracted from within each ROI. The affected area was reported to be the right 
heel; the absolute temperatures of both the right and left heels are depicted in Figure 9.16. It can be seen there is an overall similar pattern, but a deviation in specific patterns between days 14 and 62.
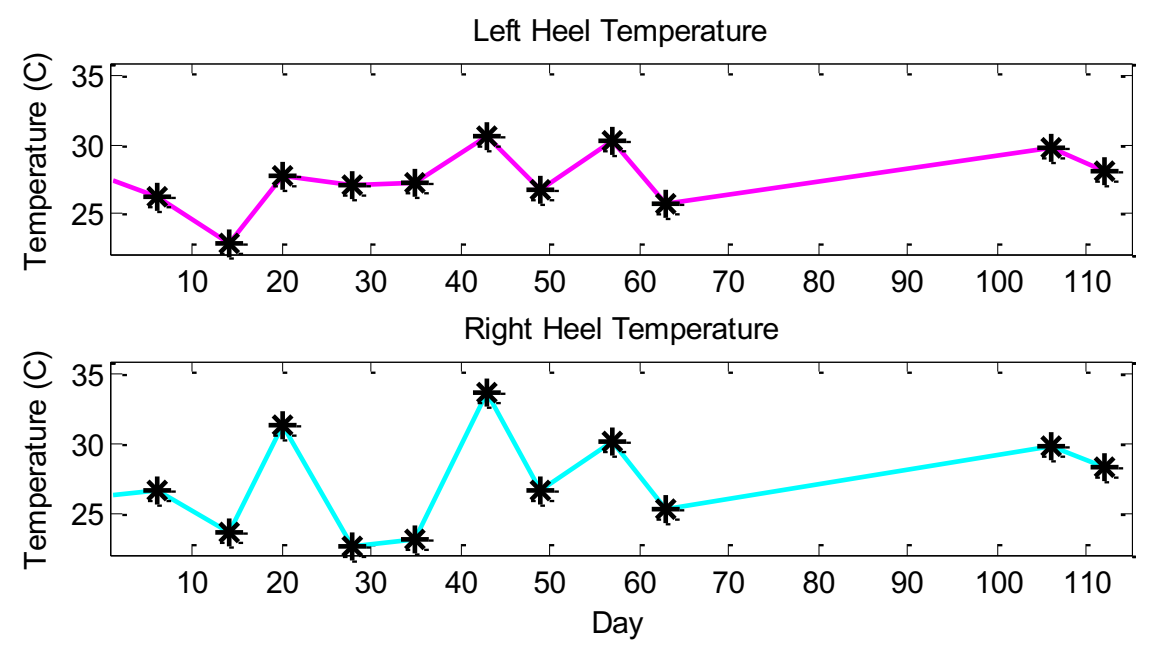

Figure 9.16 Left heel temperature compared to right heel temperature.

The difference in heel temperature between the right and left heels was calculated and is seen in Figure 9.17. This plot depicts the differences observed in Figure 9.16. Important observations corresponding to dates of data collection are seen in Table 9.2. The right heel was the patient-reported painful area. Over the first 20 days, the right heel temperature climbed to a temperature 3 degrees warmer than the left, whereas healthy heels have a very small temperature difference [23]. In addition, a small mark becomes red and inflamed. Over the next 23 days, the right heel temperature drops about 8 degrees to become 5 degrees cooler than the left heel, and then climbs rapidly back up to become 3 degrees warmer again. This pattern is consistent with the development of erythema, followed rapidly by ischemia. The spike back up at 43 days is consistent with the patterns associated with pressure relief intervention via a supportive device and the physical phenomenon of reperfusion. The observation at day 57 indicates the right heel may have experienced reperfusion injury; inflammation and oxidative 
tissue damage caused by the return of blood to tissue following a period of ischemia [134]. The heel recovers from damage due to pressure relief, and maintains a stable temperature until the last day of the study.

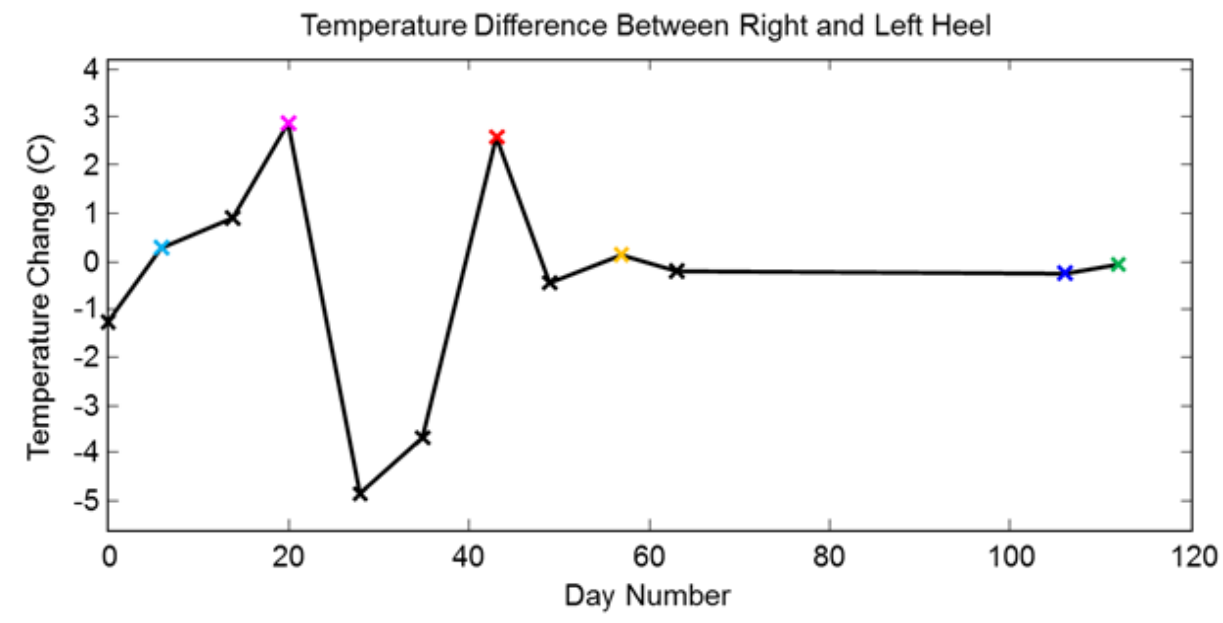

Figure 9.17 Temperature difference over time between the right and left heel

Table 9.2 Days with clinically relevant observations

\begin{tabular}{|c|c|c|}
\hline $\begin{array}{c}\text { Day } \\
\text { Number }\end{array}$ & $\begin{array}{c}\text { Marker Color } \\
\text { in Figure 5 }\end{array}$ & Observations \\
\hline 6 & $\mathbf{X}$ & $\begin{array}{c}\text { Patient complains of pain in the right } \\
\text { heel. A small mark is visible. }\end{array}$ \\
\hline 20 & $\mathbf{X}$ & $\begin{array}{c}\text { The heel and the small mark begin to } \\
\text { look red and inflamed. }\end{array}$ \\
\hline 43 & $\mathbf{X}$ & $\begin{array}{c}\text { The area surrounding the mark looks } \\
\text { worse; increasingly red and inflamed. }\end{array}$ \\
\hline 57 & $\mathbf{X}$ & $\begin{array}{c}\text { The inflamed area becomes noticeably } \\
\text { dark in contrast to the skin tone. }\end{array}$ \\
\hline 106 & $\mathbf{X}$ & $\begin{array}{c}\text { Patient no longer mentions any soreness } \\
\text { of the heel. }\end{array}$ \\
\hline 112 & Patient's skin does not display any \\
& visible redness or inflammation. \\
\hline
\end{tabular}




\subsubsection{Discussion}

This thesis examined temperature measurements in subjects and assessed these measurements over time to identify patterns related to mobility and perfusion. This section first examined thermal images of a healthy adult's hand to determine the effects of applied pressure on thermal data. Older adult patient data was then examined for thermal patterns related to mobility and blood flow over one night and long term (over three months), while also considering the variant nature of hospital data and the patterns observed in healthy adults.

Healthy adult data was gathered from one subject, who was asked to press on a pressure mat at different intensities, for five consecutive minutes. A preceding baseline thermal image was taken, and a series of pressure recovery thermal images were taken afterwards. These images were subject to standard image processing techniques before several measurements were extracted and examined. These measurements were inclusive of temperature specific contours and areas, and ROI specific intensity minimums, means, maximums and weighted centroids.

Contour plots visually displayed an overall temperature pattern; initial cooling of the hand at the areas of direct contact (fingers and base of the palm), then consequent warming of the whole hand over time to temperatures exceeding that of the baseline image. Each contour enclosed areas, or number of pixels, of different temperature levels. Areas were calculated and examined for change over time. Results confirmed the hand did heat over time; temperatures of $32.0^{\circ} \mathrm{C}$ and above increased in area while temperatures of $31.0^{\circ} \mathrm{C}$ and below decreased in area. ROI intensity measurements further validated visually observed thermal patterns; results indicated an initial cooling of the fingers followed by heating over time, while the wrist and 
palm (on average) heated more slowly over time without initial cooling. Contour area measurements alone captured an overall thermal pattern while ROI measurements captured region-specific thermal patterns.

In addition to capturing the thermal pattern of a hand as a result of applied pressure, the extraction of measurements revealed two unexpected non-visual patterns. The first being that the application of light pressure resulted in the same patterns (of comparable quantified values) as the application of heavy pressure, and the second being that while the absolute temperatures of the hand varied, the distribution of heat (represented by a weighted centroid in each ROI) barely changed over the ten minute time period.

The determination of thermal patterns in the extremities of healthy adult subjects in response to applied pressure is useful in understanding the immediate thermal response. The examination of older adult subjects allows for the observation of long term thermal patterns associated with disease and lack of mobility. Older adult thermal data was examined over one night in relation to mobility data captured using pressure mats, in order to determine a possible relationship between thermal and mobility data. One night of thermal and pressure data were examined from one at-risk older adult patient. This patient was identified to sleep in a supine position; accordingly, only thermal images of the heels and pressure data generated by the patient's feet were examined.

Thermal contours were found for each thermal image, each contour representing each $1^{\circ} \mathrm{C}$ between $23^{\circ} \mathrm{C}$ to $32^{\circ} \mathrm{C}$, as well as the pixel area enclosed by each temperature contour. Measures of comparison were employed to identify thermal patterns while excluding external influences as much as possible. The differences between the patient's left heel and right heel were visibly identifiable: the left heel (particularly the lower left heel) was significantly cooler 
than the right heel. These differences were seen in contrast to the healthy adult's heels, which displayed very similar temperature distributions in the left heel versus the right. These visually identifiable patterns were validated by the calculation of temperature area ratios. The $L / R$ temperature area ratios varied widely for the patient's heels when compared to the healthy heels, indicating the temperature distributions are more symmetrical in the healthy heels.

Pressure data generated by the patient while sleeping at night was analyzed by first calibrating data, then introducing a pressure threshold to identify times at which the patient was in bed, then averaging sensor scores over these time periods as well as calculating the standard deviation. The visual representation of this data can be found in the Mobility section, in Figure 9.5. The average exerted pressure was higher underneath the left heel than the right and the standard deviation was higher under the right heel than the left. Ratio calculations confirmed this visually identifiable pattern. When comparing results of the left heel to the right heel, it is interesting to note that the left heel was colder, while also having experienced a higher degree of applied pressure and less movement.

In the examination of long term thermal patterns of older adult subjects, thermal images were captured of one older adult patient at-risk of developing pressure ulcers, over a period of 112 days and subjecting these images to image processing, region selection and feature extraction.

The regions of interest were the left and right heels and malleoli of the patient, and the extracted feature was the mean intensity from within each ROI. Large regions were favored over small regions; while specific contour-based, topographic-based and watershed methods could be examined, the nature of the hospital and data collection means that slight changes could be the result of any number of external factors and are therefore unreliable. Considering 
larger regions and averaging within regions is more suitable for this application as the patterns are more reliable. Even though this thesis has averaged larger areas, there is a distinct temperature pattern observed that is consistent with the physical phenomenon of a stage 1 pressure ulcer as it developed and was treated. This pattern was erythema in the right heel, followed by ischemia due to unrelieved pressure, reperfusion due to relieved pressure, possible reperfusion injury and recovery. These results suggest that thermal imaging, in conjunction with image processing may be able to detect the formation of pressure ulcers faster than can be visually observed; here, the temperature rise in one heel relative to itself and the healthy heel may be an indication of ulcer formation. While these results are suggestive, this study was limited by the number of examined patients that began developing a pressure ulcer. More pressure ulcer formation data is required from numerous patients to validate the pattern observed here.

\subsubsection{Conclusion}

This thesis examined the relationship between temperature and pressure, how temperature can inform blood flow and perfusion, and the development of pressure ulcers. Data from healthy adult volunteers performing controlled experiments was first examined with the goal of identifying and quantifying thermal changes occurring in response to applied pressure. Temperature measurements of patients susceptible to the development of pressure ulcers in a hospital setting were then examined, to identify and quantify cumulative temperature changes in response to long term pressure in at-risk subjects. Long term thermal data was then examined for one 92-year-old female, who began development of a pressure ulcer over a duration of 112 days. 
The observed thermal patterns specific to the healthy adult volunteer suggest that measurement extraction can confirm and quantify visually observed thermal behaviour and additionally, capture behaviour that was not anticipated and not visually identifiable.

The observed thermal patterns specific to older adult patients first suggest a relationship between applied pressure and temperature distributions of the feet. While many factors contribute to the temperature distribution of the feet, namely health related impedance of blood flow to the extremities, a patient's in-bed behavior may also contribute. Considering the amount of time an at-risk elderly patient spends in bed (often sleeping), and the known effects of prolonged pressure on susceptible tissue, it is reasonable to consider that the temperature distribution of the feet may be related to a patient's long term sleeping behavior.

Long term thermal patterns were examined in one older adult subject who presented with signs of pressure ulcer development and recovery. Both heels were examined over 112 days and observations were quantified through thermal imaging and image processing. The results suggest that thermal imaging may be a useful tool in the monitoring of pressure ulcer development. These results also suggest that more emphasis needs to be placed on the observation of ulcer development through advanced measurement techniques, so we can better define the parameters through which we are to detect and prevent pressure ulcers. 


\section{Chapter 10}

\section{Conclusions and Future Work}

This chapter discusses the conclusions drawn from this thesis, and future directions for this research.

\subsection{Thesis Conclusions}

The main objective of this thesis was to examine, test and improve upon the current EVM processing technique and expand its use to thermal video vital signal extraction. This thesis aimed to do this within a system framework that was designed to extract vital signal measurements from in-bed subjects using three modalities in conjunction with image and signal processing. The three modalities included thermal infrared cameras, visible light cameras and pressure sensitive mats. Vital signals included pulse, respiration, temperature and mobility. Healthy adult and older adult patient subjects participated in several experimental procedures including thermal and visible light camera data capture of faces, hands and feet as well as in-bed pressure mat data capture. A system of algorithms was developed for this thesis to automatically extract vital sign measurements from a subject. This thesis aimed to not only extract vital signal measurements, but explore patterns associated with in-bed, older adult morbidities, and the ability of the proposed system to detect these abnormalities. The following subsections conclude each stage of data processing. 


\subsubsection{Data Pre-Processing and Context-Awareness}

Chapter 4 detailed data preprocessing techniques used to reduce image noise and enhance images for better vital signal extraction. Thermal images were subjected to median filtering and CLAHE enhancement, visible-light images were subjected to gaussian filtering, and pressure mat signals were countered for the weight of the mattress and then low pass filtered. These methods were successful in improving the images or signals, and consequently resulted in better vital signal measurement extraction.

Chapter 4 also examined the role of context-awareness within the system framework. The chapter used a specific instance to demonstrate the potential for context-awareness to improve the existing system framework by reducing error and processing time.

\subsubsection{Data Segmentation}

Chapter 5 detailed the methods used to identify regions corresponding to respective vital signals. Pre-processed data, the algorithms for which are detailed in Chapter 4, were used as an input to this stage of data proccesing.

Regions of interest for pulse measurement extraction were identified through several methods. Proximal pulse regions were identified in both visible-light and thermal-infrared video using binary masks placed at anatomical locations known to reveal heart rate; i.e. over major blood vessels, or areas successfully used by numerous previous studies (forehead). Distal pulse regions were considered to be regions containing a subject's arm or leg, and not the subject's chest or face. Subject feet were examined, and anatomical regions of interest were identified in both visible-light and thermal-infrared images using binary masks. Subject arms were also examined via thermal-infarred cameras. Anatomical regions of interest were 
identifed using three different methods of image segementation; basic thresholding, watershed method and level set method. Watershed method best segmented blood vessels of interest and portrayed the blood flow patterns of vascular occlusion and relief.

Respiration was performed by subjects through the nose, and examined through thermal video data. Regions of interest were therefore considered to be the area surrounding a subject's nostrils. These regions were identified using binary masks, as well as two methods that include region tracking (therefore accounting for subject motion). The first method was based on connected components analysis, and the second method was based on the Viola-Jones algorithm. Method 1 resulted in an 80\% region identification accuracy, whereas Method 2 resulted in a 90\% region identification accuracy, and a stronger respiratory signal.

Temperature in this thesis is examined in reference to mobility measurements and as temperature and mobility measurements relate to and inform pulse measurements. Temperature was examined in subject extremities through thermal image data; this included images of subject hands and feet. These images were segmented using binary masks to identify regions of interest, and using contours to segment the image by temperature. These techniques were able to reveal temporal temperature patterns related to applied pressure and consequent changes in blood flow.

Data segmentation in pressure mat data consisted of identifying regions spatially that were relevant to mobility measurements. These regions included the entire pressure mat directly under subject's feet, as well as a center of pressure measurement to attain an overall measurement of spatial movement. 
This chapter demonstrated the importance of data segmentation in the extraction of vital signals. All data segmentation methods detailed in this chapter successfully identified regions of interest for extraction of respective vital signal measurements.

\subsubsection{Video Magnification}

Chapter 6 detailed the development of an adaptive spatio-temporal video processing technique. This chapter demonstrated the potential of the proposed adaptive EVM method to extract an existing physiological signal and not simply an amplified noise signal. Furthermore, this work demonstrated the potential for thermal video in conjunction with the proposed adaptive EVM methods to be used in the extraction of physiological signals.

This chapter tested the robustness of both EVM and AEVM algorithms by generating simulated thermal videos, adding varying amounts of imposed noise to these videos and using both EVM and AEVM to extract signals and estimated heart rates. The results suggest there are several patterns associated with system failure. Identification of these patterns is important in informing future use of these algorithms; understanding and recognizing symptoms of noise, filter width or amplitude error.

The developed adaptive method was employed throughout the thesis, to extract multiple vital signals from thermal infrared and visible light video data of subjects.

\subsubsection{Pulse Measurements}

Chapter 7 applied the algorithms developed in Chapter 5 and Chapter 6 to extract pulse measurements and patterns in pulse associated with blood occlusion. Image and signal processing methods detailed in this chapter were used to estimate pulse measurements close to 
the heart (proximal measurements), pulse measurements at the extremities (distal measurements), and the effectiveness of the proposed methods in the presence of noise and signal abnormalities.

The results of this chapter demonstrated the potential of thermal video in conjunction with adaptive EVM methods to extract an underlying temperature signal representative of subject pulse, both proximally and distally, and detect and distinguish between an underlying signal and the amplification of noise. This chapter also demonstrated some advantages of Eulerian enhanced thermal videos over Eulerian enhanced visible-light videos in the extraction of vital signals; lighting conditions and subject colour have a confounding impact on visible-light data, where thermal data remains impervious.

\subsubsection{Respiration Measurements}

Chapter 8 applied the algorithms developed in Chapter 5 and Chapter 6 to extract respiration measurements and abnormalities in respiration associated with morbidities such as sleep apnea. Thermal video data was subjected to intensity analysis with and without region tracking to encompass temperature changes over time, and sum of absolute differences analysis to encompass motion over time. The results suggest that both temperature and motion methods can detect normal respiration, and that region tracking improves the accuracy of these measurements. However, one method itself is not sufficient to extract all relevant respiration information from thermal video for detection of OSA breathing; the temperature based processing better indicated the volume of airflow, whereas the motion based processing better detected absence of breath and OSA chest motion. These results enforce the notion that 
incorporating information from different physical phenomena leads to better detection of respiration patterns, particularly OSA.

\subsubsection{Mobility Measurements}

Chapter 9 examined mobility measurement extraction such as COP in the identification of subject position transitions. This chapter also examined one important in-bed mobility related health outcome; pressure ulcer development, which is related to blood flow, and as a result skin temperature. In this sense mobility and temperature are closely related, and the results of the mobility and temperature measurement analysis are complementary.

Identification and distinction of and between different in-bed movements is a first step towards the automation of in-bed mobility tools. This chapter built upon a previous algorithmic system capable of identifying and distinguishing between three different positional states and three state transfers. This chapter was able to shed light on some of the complexities of these states and state transfers through several different methods including pressure point and COP

monitoring. With further development, an algorithmic system could be implemented into a hospital setting to assist with, and eventually replace human identification and assessment of elderly patient movement; a needed hospital service for an expanding elderly population.

\subsubsection{Temperature Measurements}

Chapter 9 examined the relationship between temperature and pressure, how temperature can inform blood flow and perfusion, and the development of pressure ulcers. Data from healthy adult volunteers performing controlled experiments was first examined with the goal of identifying and quantifying thermal changes occurring in response to applied pressure. 
Temperature measurements of patients susceptible to the development of pressure ulcers in a hospital setting were then examined, to identify and quantify cumulative temperature changes in response to long term pressure in at-risk subjects. Long term thermal data was then examined for one 92-year-old female, who began development of a pressure ulcer over a duration of 112 days.

The observed thermal patterns specific to the healthy adult volunteer suggest that measurement extraction can confirm and quantify visually observed thermal behaviour and additionally, capture behaviour that was not anticipated and not visually identifiable.

The observed thermal patterns specific to older adult patients first suggest a relationship between applied pressure and temperature distributions of the feet. While many factors contribute to the temperature distribution of the feet, namely health related impedance of blood flow to the extremities, a patient's in-bed behavior may also contribute. Considering the amount of time an at-risk elderly patient spends in bed (often sleeping), and the known effects of prolonged pressure on susceptible tissue, it is reasonable to consider that the temperature distribution of the feet may be related to a patient's long term sleeping behavior.

Long term thermal patterns were examined in one older adult subject who presented with signs of pressure ulcer development and recovery. Both heels were examined over 112 days and observations were quantified through thermal imaging and image processing. The results suggest that thermal imaging may be a useful tool in the monitoring of pressure ulcer development. These results also suggest that more emphasis needs to be placed on the observation of ulcer development through advanced measurement techniques, so we can better define the parameters through which we are to detect and prevent pressure ulcers. 


\subsection{Thesis Contributions}

In addition to the final conclusions, this thesis has provided four main contributions. First, in the examination of COP as a mobility measure; the work completed in this thesis demonstrated the potential for COP to be informative in patient mobility. The second contribution being in image segmentation; extensive work was completed on image segmentation of various body parts, including advanced techniques such as level set method, watershed method and region tracking for vital signal measurements, allowing for more accurate measurements. The third contribution builds upon previous work by expanding the experimental scope of current research, as well as developing a method called the Adaptive Eulerian Video Magnification (AEVM) method, that addresses some shortcomings of the EVM algorithm. The use of this algorithm allowed for the extraction of respiration and pulse measurements proximally, distally and from both thermal infrared and visible-light cameras. The fourth contribution was the examination of temperature and mobility measurements from in-bed subjects independently, and in relation to each other. This contribution is valuable, as the data examined originates from older adult in-hospital patients and therefore speaks directly to morbidities related to this co-hort.

\subsection{Future Work}

This thesis worked towards a non-contact system that would extract vital signals from a subject in bed. This system would be of particular use to such applications as at-home monitoring, remote health monitoring, or to be incorporated to hospital environments to assist in more consistent measurements. While this thesis examined both older adult in-hospital patients and healthy adult volunteers, the population likely to most benefit from such a system would be older adult subjects who are good candidates for at home health monitoring. 
The framework developed for this thesis was developed with the intention of expansion through continued research. This thesis would benefit first from testing with more subjects, and with more iterations per subject to gather more data regarding accuracy, specificity and repeatability as well as explore the clinical significance of these metrics. One of the most relevant topics of exploration would be the addition of fusion. The fusion of information from different physical phenomena could include fusion of visible-light image data and thermal image data, or fusion of motion-based thermal measurements and thermal-based thermal measurements, both of which could improve vital signal measurements by increasing reliability and reducing error. Future work also involves more subjects, both healthy adult and older adult patient subjects, as well as the further testing of and compensation for significant noise, interference and motion.

Future work therefore involves the examination and comparison of other temperaturebased and motion-based methods, in addition to facial recognition and tracking methods, followed by the examination of fusion methods. Additional future work involves testing fusion algorithms with many more subjects and testing and compensation of significant noise, interference and motion. 


\section{References}

1. M. Caley, and S. Khesh, "Estimating the future healthcare costs of an aging population in the UK: expansion of morbidity and the need for preventative care," Journal of Public Health, vol. 33, no. 1, pp. 117-122, June 2010.

2. C. Brüser, C. H. Antink, T. Wartzek, M. Walter, and S. Leonhardt, “Ambient and Unobtrusive Cardiorespiratory Monitoring Techniques," IEEE Reviews in Biomedical Engineering, vol. 8, pp. 30-43, 2015.

3. Oxford Concise Medical Dictionary, 9 ed. (2015), 'vital signs' entry. Online. http://www.oxfordreference.com.proxy.library.carleton.ca/view/10.1093/acref/978019 9687817.001.0001/acref-9780199687817-e-11968?rskey=YF9jbX\&result=12185

4. M. Brabrand et al, "Should impaired mobility on presentation be a vital sign?" Acta Anaesthesiologica Scandinavica, vol. 62, (7), pp. 945-952, 2018.

5. I. J. Brekke et al, "The value of vital sign trends in predicting and monitoring clinical deterioration: A systematic review," PloS One, vol. 14, (1), pp. e0210875, 2019.

6. J. Kellett and F. Sebat, "Make vital signs great again - A call for action," European Journal of Internal Medicine, vol. 45, pp. 13-19, 2017. 
7. H. J. Kellett et al, "Changes and their prognostic implications in the abbreviated Vitalpac $^{\mathrm{TM}}$ early warning score (ViEWS) after admission to hospital of 18,853 acutely ill medical patients," Resuscitation, vol. 84, (1), pp. 13-20, 2012;2013.

8. M. Cardona-Morrell et al, "Vital signs monitoring and nurse-patient interaction: A qualitative observational study of hospital practice," International Journal of Nursing Studies, vol. 56, pp. 9-16, 2016.

9. H. Y. Wu, M. Rubinstein, E. Shih, J. Guttag, F. Durand, and W. Freeman, "Eulerian video magnification for revealing subtle changes in the world," ACM Trans. on Graphics (TOG), vol. 31, no. 4, pp. 651-658, July 2012.

10. M. Vrbaski and D. Petriu, "Toward a context awareness framework for healthcare applications," in 2013 IEEE International Symposium on Medical Measurements and Applications (MeMeA), 2013, pp. 339-344.

11. E. Aguirre et al., "Implementing context aware scenarios to enable smart health in complex urban environments," in 2014 IEEE International Symposium on Medical Measurements and Applications (MeMeA), 2014, pp. 1-4.

12. S. Bennett, Z. Ren, R. Goubran, K. Rockwood, and F. Knoefel, "In-Bed Mobility Monitoring Using Pressure Sensors," in IEEE Trans. Instrum. Meas., vol. 64, no. 8, May 2015, pp. 2110-2120. 
13. S. Bennett, R. Goubran, and F. Knoefel, "Comparison of Motion-Based Analysis to Thermal-Based Analysis of Thermal Video in the Extraction of Respiration Patterns," in Proc. IEEE Int. Conf. Eng. Med. Biol. Soc. (EMBC), Jeju Island, South Korea, July 2017, pp. 1-5.

14. S. L. Bennett, R. Goubran, and F. Knoefel, "Examining Changes in Image Segmented Arteries in Response to Blood Occlusion," in Proc. IEEE Med. Meas. Appl. (MeMeA), Rochester, Minnesota, USA, May 2017, pp. 1-5.

15. S. L. Bennett, R. Goubran, and F. Knoefel, "Examining the Effect of Noise on Biosignal Estimates Extracted through Spatio-Temporal Video Processing," accepted to Proc. IEEE Int. Conf. Eng. Med. Biol. Soc. (EMBC), Berlin, Germany, June 2019, pp. 1-5.

16. S. L. Bennett, R. Goubran, and F. Knoefel, "Context-awareness in non-contact, multimodality, bed-based monitoring of vital signs," in Proc. IEEE Med. Meas. Appl. (MeMeA), Rome, Italy, June 2018, pp. 1-5.

17. X. He et. al., "Video-based analysis of heart rate applied to falls," in Proc. IEEE Med. Meas. Appl. (MeMeA), Rome, Italy, June 2018, pp. 1-5.

18. S. Bennett, T. N. El Harake, R. Goubran, and F. Knoefel, “Adaptive Eulerian Video Processing of Thermal Video: An Experimental Analysis," in IEEE Trans. Instrum. Meas., April 2017, pp. 1-9. 
19. S. L. Bennett, R. Goubran, B. Bennett, R. Bennett, and F. Knoefel, "The Use of a Thermal Camera and Eulerian Enhancement in the Examination of Pedal Pulse and Microvascular Health," in Proc. IEEE Int. Conf. Eng. Med. Biol. Soc. (EMBC), Orlando, Florida, August 2016, pp. 1-4.

20. S. L. Bennett, R. Goubran, and F. Knoefel, "Adaptive Eulerian Video Magnification Methods to Extract Heart Rate From Thermal Video," in Proc. IEEE Med. Meas. Appl. (MeMeA), Benevento, Italy, May 2016, pp. 1-5.

21. S. L. Bennett, R. Goubran, and F. Knoefel, "The Detection of Breathing Behavior Using Eulerian-Enhanced Thermal Video," in Proc. IEEE Int. Conf. Eng. Med. Biol. Soc. (EMBC), Milan, Italy, August 2015, pp. 7474-7477.

22. S. Bennett, R. Goubran, and F. Knoefel, "Long Term Monitoring of a Pressure Ulcer Risk Patient Using Thermal Images," in Proc. IEEE Int. Conf. Eng. Med. Biol. Soc. (EMBC), Jeju Island, South Korea, July 2017, pp. 1-4.

23. S. L. Bennett, R. Goubran, and F. Knoefel, "Measurement and Comparison of Thermal and In-Bed Pressure Data towards the Prevention Pressure Ulcers," in Proc. IEEE Int. Conf. Biomed. Health Informatics (BHI), Las Vegas, USA, February 2016, pp. 62-65.

24. S. L. Bennett, R. Goubran, and F. Knoefel, "Measurements of Change in Thermal Images Due to Applied Pressure," in Proc. IEEE Med. Meas. Appl. (MeMeA), Turin, Italy, May 2015, pp. 30-35. 
25. M. Mabrouk, S. Rajan, M. Bolic, I. Batkin, H. R. Dajani, and V. Z. Groza, "Model of human breathing reflected signal received by PN-UWB radar," in 2014 36th Annual International Conference of the IEEE Engineering in Medicine and Biology Society (EMBC), 2014, pp. 4559-4562.

26. G. Sun and T. Matsui, "Rapid and stable measurement of respiratory rate from Doppler radar signals using time domain autocorrelation model," in 2015 37th Annual International Conference of the IEEE Engineering in Medicine and Biology Society (EMBC), 2015, pp. 5985-5988.

27. M. Varanini, P. C. Berardi, F. Conforti, M. Micalizzi, D. Neglia, and A. Macerata, "Cardiac and respiratory monitoring through non-invasive and contactless radar technique," in 2008 Computers in Cardiology, 2008, pp. 149-152.

28. L. N. Anishchenko, "Independent component analysis in bioradar data processing," in 2016 Progress in Electromagnetic Research Symposium (PIERS), 2016, pp. 2206-2210.

29. Z. Zhang et al., "Multimodal Spontaneous Emotion Corpus for Human Behavior Analysis," in 2016 IEEE Conference on Computer Vision and Pattern Recognition (CVPR), 2016, pp. 3438-3446.

30. B. Xu, H. Madhu, R. Kulkarni, L. K. Mestha, S. Kyal, and G. Pennington, "Evaluating and improving the robustness of a video-based $\mathrm{PR} / \mathrm{RR}$ monitoring system for a clinical environment," in 2016 IEEE Healthcare Innovation Point-Of-Care Technologies Conference (HI-POCT), 2016, pp. 204-207. 
31. M.-Z. Poh, D. J. McDuff, and R. W. Picard, "Advancements in Noncontact, Multiparameter Physiological Measurements Using a Webcam," IEEE Transactions on Biomedical Engineering, vol. 58, no. 1, pp. 7-11, 2011.

32. D. M. Tveit, K. Engan, I. Austvoll, and Ø. Meinich-Bache, "Motion based detection of respiration rate in infants using video," in 2016 IEEE International Conference on Image Processing (ICIP), 2016, pp. 1225-1229.

33. R. Anusree, R. Ranjith, and K. I. Ramachandran, "A noncontact vital sign monitoring algorithm using a camera," in 2016 International Conference on Advances in Computing, Communications and Informatics (ICACCI), 2016, pp. 1947-1951.

34. T. R. Gault, N. Blumenthal, A. A. Farag, and T. Starr, "Extraction of the superficial facial vasculature, vital signs waveforms and rates using thermal imaging," in 2010 IEEE Computer Society Conference on Computer Vision and Pattern Recognition Workshops, 2010, pp. 1-8.

35. F. Q. AL-Khalidi, R. Saatchi, D. Burke, and H. Elphick, "Tracking human face features in thermal images for respiration monitoring," in ACS/IEEE International Conference on Computer Systems and Applications - AICCSA, 2010, pp. 1-6.

36. B. Aubakir, B. Nurimbetov, I. Tursynbek, and H. A. Varol, "Vital sign monitoring utilizing Eulerian video magnification and thermography," in 2016 38th Annual International Conference of the IEEE Engineering in Medicine and Biology Society (EMBC), 2016, pp. 3527-3530. 
37. C. W. Wang, A. Hunter, N. Gravill, and S. Matusiewicz, "Unconstrained Video Monitoring of Breathing Behavior and Application to Diagnosis of Sleep Apnea," IEEE Transactions on Biomedical Engineering, vol. 61, no. 2, pp. 396-404, Feb. 2014.

38. V. H. Cheung, L. Gray and M. Karunanithi, "Review of accelerometry for determining daily activity among elderly patients," Archives of Physical Medicine and Rehabilitation, vol. 92, no. 6, pp. 998-1014, 2011.

39. D. I. Townsend, R. Goubran, M. Frize, and F. Knoefel, "Preliminary results on the effect of sensor position on unobtrusive rollover detection for sleep monitoring in smart homes," in Proc. IEEE Eng. Med. Biol. Soc., pp. 6135-6138, Sept. 2009.

40. M. H. Jones, R. Goubran and F. Knoefel, "Identifying movement onset times for a a bedbased pressure sensor array," in Proc. IEEE Med. Meas. Appl. (MeMeA), pp. 111-114, April 2006.

41. M. H. Jones, R. Goubran and F. Knoefel, "Reliable Respiratory Rate Estimation from a Bed Pressure Array," in Proc. IEEE Eng. Med. Biol. Soc. pp. 6410-6413, 2006.

42. V. Mishra, N. Singh, U. Tiwari and P. Kapur, "Fiber Grating Sensors in Medicine: Current and Emerging Applications," Sensors \& Actuators: A. Physical, vol. 167, no. 2, pp. 279-290, 2011. 
43. P. Buddharaju, I. Pavlidis, P. Tsiamyrtzis, and M. Bazakos, "Physiology-Based Face Recognition in the Thermal Infrared Spectrum," IEEE Transactions on Pattern Analysis and Machine Intelligence, vol. 29, no. 4, pp. 613-626, 2007.

44. S. Poeggel et al, "Optical Fibre Pressure Sensors in Medical Applications," Sensors, (Basel, Switzerland), vol. 15, (7), pp. 17115-17148, 2015.

45. A. Lay-Ekuakille, P. Vergallo, F. Conversano, S. Casciaro, and D. Veneziano, "Thermal image processing for accurate realtime decision making in surgery," in 2014 IEEE International Symposium on Medical Measurements and Applications (MeMeA), 2014, pp. $1-4$.

46. J. B. Mercer and L. de Weerd, "Thermography and thermal symmetry," in 2014 IEEE International Symposium on Medical Measurements and Applications (MeMeA), 2014, pp. 1-3.

47. L. Wang and G. Leedham, "Gray-Scale Skeletonization of Thermal Vein Patterns Using the Watershed Algorithm in Vein Pattern Biometrics, " in 2006 International Conference on Computational Intelligence and Security, 2006, vol. 2, pp. 1597-1602.

48. Z.-H. Xie, S.-Q. Wu, G.-D. Liu, and Z.-J. Fang, "Infrared face recognition method based on blood perfusion image and Curvelet transformation," in 2009 International Conference on Wavelet Analysis and Pattern Recognition, 2009, pp. 360-364. 
49. M. Akhloufi and A. Bendada, "Thermal Faceprint: A New Thermal Face Signature Extraction for Infrared Face Recognition," in 2008 Canadian Conference on Computer and Robot Vision, 2008, pp. 269-272.

50. N. Sun, M. Garbey, A. Merla, and I. Pavlidis, "Imaging the cardiovascular pulse," in 2005 IEEE Computer Society Conference on Computer Vision and Pattern Recognition (CVPR'05), 2005, vol. 2, pp. 416-421 vol. 2.

51. M. Yang, Q. Liu, T. Turner, and Y. Wu, "Vital sign estimation from passive thermal video," in 2008 IEEE Conference on Computer Vision and Pattern Recognition, 2008, pp. 1-8.

52. A. M. Mendonca and A. Campilho, "Segmentation of retinal blood vessels by combining the detection of centerlines and morphological reconstruction," IEEE Trans. on Medical Imaging, vol. 25, no. 9, pp. 1200-1213, Sep. 2006.

53. V. Bevilacqua et al., "A novel approach to evaluate blood parameters using computer vision techniques," in 2016 IEEE International Symposium on Medical Measurements and Applications (MeMeA), 2016, pp. 1-6.

54. L. Carnimeo, A. R. Altomare, and R. Nitti, "Monitoring of retinal vessels for diabetic patients in home care assistance," in 2016 IEEE International Symposium on Medical Measurements and Applications (MeMeA), 2016, pp. 1-6. 
55. F. C. F. Chui, I. Bindoff, R. Williams, and B. H. Kang, "Feature Extraction for Classification from Images: A Look at the Retina," in 2008 International Symposium on Ubiquitous Multimedia Computing, 2008, pp. 93-98.

56. B. Dashtbozorg, A. M. Mendonça, and A. Campilho, "An Automatic Graph-Based Approach for Artery/Vein Classification in Retinal Images," IEEE Transactions on Image Processing, vol. 23, no. 3, pp. 1073-1083, Mar. 2014.

57. H. Betaouaf and A. Bessaid, "A biometric identification algorithm based on retinal blood vessels segmentation using watershed transformation," in 2013 8th International Workshop on Systems, Signal Processing and their Applications (WoSSPA), 2013, pp. $256-261$.

58. B. Dizdaro, E. Ataer-Cansizoglu, J. Kalpathy-Cramer, K. Keck, M. F. Chiang, and D. Erdogmus, "Level sets for retinal vasculature segmentation using seeds from ridges and edges from phase maps," in 2012 IEEE International Workshop on Machine Learning for Signal Processing, 2012, pp. 1-6.

59. A. Wu, Z. Xu, M. Gao, M. Buty, and D. J. Mollura, "Deep vessel tracking: A generalized probabilistic approach via deep learning," in 2016 IEEE 13th International Symposium on Biomedical Imaging (ISBI), 2016, pp. 1363-1367.

60. S. Ahmad, M. Bolic, H. Dajani, V. Groza, I. Batkin, and S. Rajan, "Measurement of Heart Rate Variability Using an Oscillometric Blood Pressure Monitor," IEEE 
Transactions on Instrumentation and Measurement, vol. 59, no. 10, pp. 2575-2590, Oct. 2010.

61. S. Chen, M. Bolic, V. Z. Groza, H. R. Dajani, I. Batkin, and S. Rajan, "Extraction of Breathing Signal and Suppression of Its Effects in Oscillometric Blood Pressure Measurement," IEEE Transactions on Instrumentation and Measurement, vol. 60, no. 5, pp. 1741-1750, May 2011.

62. A. P. Twinanda, E. O. Alkan, A. Gangi, and M. de Mathelin, "Data-driven spatiotemporal RGBD feature encoding for action recognition in operating rooms," International Journal of Computer Assisted Radiology and Surgery, vol. 10, no. 6, pp. 737-747, Jun. 2015.

63. N. Sharma, A. Dhall, T. Gedeon, and R. Goecke, "Thermal spatio-temporal data for stress recognition,” J Image Video Proc, vol. 2014, no. 1, p. 28, Jun. 2014.

64. A. Sarkar, A. L. Abbott, and Z. Doerzaph, "Assessment of psychophysiological characteristics using heart rate from naturalistic face video data," in IEEE International Joint Conference on Biometrics, 2014, pp. 1-6.

65. E. Çağlayan and T. E. Özkurt, "Spectral features of heart rate variability obtained from a video camera," in 2015 23nd Signal Processing and Communications Applications Conference (SIU), 2015, pp. 955-957. 
66. X. He, R. A. Goubran, and X. P. Liu, "Using Eulerian video magnification framework to measure pulse transit time," in Proc. IEEE Int. Symp. on Medical Measurement and Applications (MeMeA), Lisbon, Portugal, pp.1-4, June 2014.

67. D. Alinovi, L. Cattani, G. Ferrari, F. Pisani, and R. Raheli, "Spatio-temporal video processing for respiratory rate estimation," in Proc. IEEE Int. Symp. on Medical Measurement and Applications (MeMeA), Turin, Italy, pp.12-17, May 2015.

68. K. Hamedani, Z. Bahmani, and A. Mohammadian, "Spatio-temporal filtering of thermal video sequences for heart rate estimation," Expert Systems with Applications, vol. 54, pp. 88-94, Jul. 2016.

69. C. Kargel, "Infrared thermal imaging to measure local temperature rises caused by handheld mobile phones," IEEE Transactions on Instrumentation and Measurement, vol. 54, no. 4, pp. 1513-1519, Aug. 2005.

70. J. G. Wang and E. Sung, "Facial Feature Extraction in an Infrared Image by Proxy With a Visible Face Image," IEEE Transactions on Instrumentation and Measurement, vol. 56, no. 5, pp. 2057-2066, Oct. 2007.

71. J. Nikolic-Popovic and R. Goubran, "Impact of motion artifacts on video-based nonintrusive heart rate measurement," in 2016 IEEE International Symposium on Medical Measurements and Applications (MeMeA), 2016, pp. 1-6. 
72. M. M. Churpek, R. Adhikari and D. P. Edelson, "The value of vital sign trends for detecting clinical deterioration on the wards," Resuscitation, vol. 102, pp. 1-5, 2016.

73. Y. M. Golightly et al, "Physical Activity as a Vital Sign: A Systematic Review," Preventing Chronic Disease, vol. 14, pp. E123, 2017.

74. H. Arkin, L. X. Xu, and K. R. Holmes, "Recent developments in modeling heat transfer in blood perfused tissues," IEEE Transactions on Biomedical Engineering, vol. 41, no. 2, pp. 97-107, 1994.

75. B. Kundu and D. Dewanjee, "A new method for non-Fourier thermal response in a single layer skin tissue," Case Studies in Thermal Engineering, vol. 5, pp. 79-88, Mar. 2015.

76. K. C. Liu, "Thermal propagation analysis for living tissue with surface heating," International Journal of Thermal Sciences, vol. 47, no. 5, pp. 507-513, 2008.

77. F. Luyster, J. Strollo, J. Patrick, P. Zee, and J. Walsh, "Sleep: a health imperative," Sleep, vol. 35, no. 6, pp. 727-734, Feb. 2012.

78. D. Buysse, "Sleep health: can we define it? Does it matter?," Sleep, vol. 37, no. 1, pp. 917C, 2014.

79. N. Wolkove, O. Elkholy, M. Baltzan, and M. Palayew, "Sleep and aging: 1. Sleep disorders commonly found in older people," Can. Med. Assoc. J., vol. 176, no. 9, pp. 1299-1304, 2007. 
80. Katherine A. Dudley, Sanjay R. Patel, "Disparities and genetic risk factors in obstructive sleep apnea," Sleep Medicine, vol. 18, pp. 96-102, Feb 2016.

81. R. Germany, S. Joseph, K. James and A. Kao, "A novel therapeutic approach for the treatment of central sleep apnea: The remedēe system," Cardiovasc. Revasc. Med., vol. 15, no. 4, pp. 235-239, 2014.

82. O. Lyons, and C. Ryan, "Sleep Apnea and Stroke," Can. J. of Cardiology, vol. 31, no. 7, pp. 918-927, 2015.

83. E. Pino, A. Dorner, P. Aqueveque, J. Chavez, and A. Moran, "Contact pressure monitoring device for sleep studies," Conf. Proc. IEEE Eng. Med. Biol. Soc., Osaka, Japan, July 2013, pp. 4160-4163.

84. G. Guerrero-Mora, P. Elvia, A. Bianchi, J. Kortelainen, M. Tenhunen, S. Himanen et. al., "Sleep-wake detection based on respiratory signal acquired through a Pressure Bed Sensor," Conf. Proc. IEEE Eng. Med. Biol. Soc., California, USA, August 2012, pp. $3452-3455$.

85. L. Walsh, E. Moloney, and S. McLoone, "Identification of nocturnal movements during sleep using the non-contact under mattress bed sensor," Conf. Proc. IEEE Eng. Med. Biol. Soc., Boston, USA, August 2011, pp. 1660-1663. 
86. D. Austin, Z. Beattie, T. Riley, A. Adami, C. Hagen, and T. Hayes, "Unobtrusive classification of sleep and wakefulness using load cells under the bed," Conf. Proc. IEEE Eng. Med. Biol. Soc., California, USA, August 2012, pp. 5254-5257.

87. R. A. Epperly, G. E. Heberlein, and L. G. Eads, "Thermography, a tool for reliability and safety," IEEE Ind. Appl. Mag., vol. 5, no. 1, pp. 28-36, Feb 1999.

88. I. A. Nola, K. Gotovac, and D. Kolaric, "Thermography in biomedicine - Specific requirements," in Proc. IEEE Int. Symp. ELMAR, Zadar, Croatia, pp.355-357, Sept. 2012.

89. V. Agostini, A. Ala, R. Bussone, A. Ciarlini, S. Delsanto, F. Molinari et. al. "Dynamic infrared imaging for breast cancer diagnosis: a feature based registration approach," in Proc. IEEE Int. Symp. on Medical Measurement and Applications (MeMeA), Benevento, Italy, pp. 58-62, April 2006.

90. N. M. Mohan, and V. J. Kumar, "Virtual instrument for assessing dermal perfusion," in Proc. IEEE Int. Symp. On Instrumentation and Measurement Technology (I2MTC), Singapore, pp.1449-1452, May 2009.

91. J. B. Mercer, and L. de Weerd, "Thermography and thermal symmetry," in Proc. IEEE Int. Symp. on Medical Measurement and Applications (MeMeA), Lisbon, Portugal, pp. 1-3, June 2014. 
92. D. S. Haddad, E. S. Arita, J. C. Pinho, M. L. Brioschi, J. Gabriel, and R. Vardasca, "The facial thermal effect of dynamic mechanical and vascular provocation tests: Preliminary study," in Proc. IEEE Int. Symp. on Medical Measurement and Applications (MeMeA), Lisbon, Portugal, pp. 1-4, June 2014.

93. A. Seixas, R. Vardasca, and J. Gabriel, "The effect of different vibration frequencies in the skin temperature in healthy subjects," in Proc. IEEE Int. Symp. on Medical Measurement and Applications (MeMeA), Lisbon, Portugal, pp. 1-5, June 2014.

94. D. Haluzan, I. Dobric, J. Stipic, T. Ehrenfreund, G. Augustin, and S. Davila, "Application of infrared thermography during bone healing," in Proc. IEEE Int. Symp. ELMAR, Zadar, Croatia, pp. 353-354, Sept. 2012.

95. J. Leachtenauer, S. Kell, B. Turner, C. Newcomer, C. Lyder, and M. Alwan, "A NonContact Imaging-Based Approach to Detecting Stage I Pressure Ulcers," in Proc. IEEE Int. Conf. Eng. Med. Biol. Soc., New York City, New York, pp. 6380-6383, Sept. 2006.

96. E. S. Papazoglou, M. S. Weingarten, L. Zubkov, M. Neidrauer, and K. Pourrezaei, "Assessment of diabetic foot ulcers with diffuse near infrared methodology," in Proc. IEEE Int. Conf. BioInformatics and BioEngineering, (BIBE), pp. 1-5, Oct. 2008.

97. J. R. Harding, D. F. Wertheim, R. J. Williams, J. M. Melhuish, D. Banerjee, and K. G. Harding, "Infrared imaging in diabetic foot ulceration," in Proc. IEEE Int. Conf. Eng. Med. Biol. Soc., Hong Kong, China, pp. 916-918, Oct 1998. 
98. C. Toledo, F. J. Ramos, J. Gutierrez, A. Vera, and L. Leija, "Non-invasive imaging techniques to assess diabetic foot ulcers: A state of the art review," in Proc. IEEE Int. Conf. Pan American Health Care Exchanges (PAHCE), Brasilia, Brazil, pp. 1-4, April 2014.

99. K. Ammer, P. Melnizky, O. Rathkolb, and E. F. Ring, "Thermal imaging of skin changes on the feet of type II diabetics," in Proc. IEEE Int. Conf. Eng. Med. Biol. Soc., Istanbul, Turkey, pp. 2870-2872, 2001.

100. S. Bennett, R. Goubran, K. Rockwood, and F Knoefel, "Monitoring the Relief of Pressure Points for Pressure Ulcer Prevention: A Subject Dependant Approach," in Proc. IEEE Int. Symp. on Medical Measurements and Applicat. (MeMeA), Gatineau, QC, pp. 135-138, 2013.

101. S. Bennett, R. Goubran, K. Rockwood, A. Arcelus, and F Knoefel, "Pressure Signal Feature Extraction for the Differentiation of Clinical Mobility Assessments," in Proc. IEEE Med. Meas. Appl. (MeMeA), Budapest, Hungary, pp. 1-5, May 2012.

102. N. Foubert, A. McKee, R. Goubran, and F. Knoefel, "Lying and sitting posture recognition and transition detection using a pressure sensor array," in Proc. IEEE Int. Symp. on Medical Measurements and Applicat. (MeMeA), Budapest, Hungary, pp. 1-6, May 2012. 
103. N. Foubert, "Posture recognition and postural transition detection using bed-based pressure sensor arrays,” M.A.Sc. thesis, Dept. of Computer and Systems Engineering, Carleton University, Ottawa, ON, 2010.

104. A. Arcelus, R. Goubran, F. Knoefel, H. Sveistrup, and M. Bilodeau, "Detection of bouncing during sit-to-stand transfers with sequential pressure images," in Proc. IEEE Med. Meas. Appl. (MeMeA), Bari, Italy, pp. 158-161, May 2011.

105. A. Arcelus, I. Veledar, R. Goubran, F. Knoefel, M. Bilodeau, and H. Sveistrup, "Measurements of sit-to-stand timing and symmetry from bed pressure sensors,' IEEE Trans. Instrum. Meas., vol. 60, no. 5, pp. 1732-1740, May 2011.

106. E. Marenzi, G. M. Bertolotti and A. Cristiani, "Design and Development of a Monitoring System for the Interface Pressure Measurement of Seated People," IEEE Trans. Instrum. Meas., vol. 62, no. 3, pp. 570-577, Mar. 2013.

107. J. P. Ferreira, M. M. Crisostomo and A. P. Coimbra, "Human Gait Acquisition and Characterization," Instrumentation and Measurement, IEEE Transactions on , vol. 58, no. 9, pp. 2979-2988, Sept. 2009.

108. S. Bennett, R. Goubran, K. Rockwood, and F Knoefel, "Distinguishing between stable and unstable sit-to-stand transfers using pressure sensors," in Proc. IEEE Med. Meas. Appl. (MeMeA), Lisbon, Portugal, pp. 76-80, June 2014. 
109. Z. Ren, T. Grant, R. Goubran, M. El-Tanany, F. Knoefel, H. Sveistrup, M. Bilodeau, and J. Jutai, "Analyzing center of pressure progression during bed exits," in Proc. IEEE Eng. Med. Biol. Soc. (EMBS), Chicago, IL, pp. 1786-1789, Aug. 2014.

110. L. H. Griswold et al, "Validity of the Braden Scale in grading pressure ulcers in trauma and burn patients," Journal of Surgical Research, vol. 219, pp. 151-157, 2017.

111. FLIR: R\&D and Science. Infrared Camera Accuracy and Uncertainty in Plain Language [website]. https://www.flir.ca/discover/rd-science/infrared-camera-accuracy-anduncertainty-in-plain-language/

112. FLIR: Support: Products. FLIR E4 [website]. https://www.flir.com/products/e4 \#Overview

113. FLIR: Support: Products. FLIR t1030sc [website]. https://www.flir.com/support/products/t1030sc\#Specificatio

114. Hoskin Scientifique: Instrumentation: FLIR A40M [website]. http://www.hoskinscientifique.com/uploadpdf/Instrumentation/FLIR\%20Systems/hosk in_A40_researcher_478b84ce59aad.pdf

115. American Academy of Thermology. Guidelines for Neuro-musculoskeletal infrared medical thermography and sympathetic skin response (SSR) studies [website]. https://aathermology.org/organization-2/guidelines/guidelines-for-neuromusculoskeletal-thermography/ 
116. International Academy of Clinical Thermology. Thermology Guidelines: Standards and Protocols in Clinical Thermographic Imaging [website]. http://www.iactorg.org/professionals/thermog-guidelines.html

117.FLIR - 5 Factors influencing radiometric temperature measurements. [website] http://www.cvl.isy.liu.se/education/undergraduate/tsbb09/lectures/Guidebook_Cores_5 _Factors_Influencing_Radiometric_Temperature_Measurements

118. $\mathrm{Y} . \mathrm{Wu}$ and SpringerLink (Online service), Knee joint vibroarthrographic signal processing and analysis, 2015th ed. Heidelberg: Springer, 2015.

119. R. Gade and T. B. Moeslund, "Thermal cameras and applications: a survey," Machine Vision and Applications, vol. 25, no. 1, pp. 245-262, Jan. 2014.

120. M. Farooque and J. Rohankar, "Survey on various noises and techniques for denoising the color image.," International Journal of Application or Innovation in Engineering \& Management (IJAIEM), vol. 2, no. 11, pp. 217-221, Nov. 2013.

121. M. Holtzman, D. Townsend, R. Goubran, and F. Knoefel, "Validation of pressure sensors for physiological monitoring in home environments," in 2010 IEEE International Workshop on Medical Measurements and Applications, 2010, pp. 38-42.

122. P. Ferreira, P. Alves and SpringerLink (Online service), Distributed Context-Aware Systems. (1;2014; ed.) 2014. DOI: 10.1007/978-3-319-04882-6. 
123. A. I. Arcelus, Carleton University. Theses and Dissertations. Engineering, Electrical and Computer, "Context-Aware Smart Home Monitoring through the Analysis of Pressure Sequences," ProQuest Dissertations Publishing, Ottawa, 2010.

124. C. Li, C. Xu, C. Gui, and M. D. Fox, "Distance Regularized Level Set Evolution and Its Application to Image Segmentation," IEEE Transactions on Image Processing, vol. 19, no. 12, pp. 3243-3254, Dec. 2010.

125. The Royal Marsden Manual of Clinical Nursing Procedures: Ninth Edition. [website]. http://www.royalmarsdenmanual.com/student/flashcards/c10_1/c10_1.htm

126. A. Peretz et al, "Flow mediated dilation of the brachial artery: An investigation of methods requiring further standardization," BMC Cardiovascular Disorders, vol. 7, (1), pp. 11-11, 2007.

127. E. Winarno et al, "Multi-View Faces Detection Using Viola-Jones Method," Journal of Physics: Conference Series, vol. 1114, pp. 12068, 2018.

128. R. Raghavendra, M. Avinash, S. Marcel, and C. Busch, "Finger vein liveness detection using motion magnification," in Proc. IEEE Int. Symp. On Biometrics Theory, Applications and Systems (BTAS), pp.1-7, September 2015.

129. W. Neal, M. Rubinstein, F. Durand, and W. T. Freeman, "Phase-based video motion processing," ACM Trans. Graph., vol. 32, no. 4, pp. 1-9, July 2013. 
130. L. Liu, L. Lu, L. Jingjing, J. Zhang, and X. Chen, "Enhanced Eulerian video magnification," in Proc. IEEE Int. Congress on Image and Signal Processing (CISP), pp.50-54, Oct. 2014.

131. Tulyakov, X. Alameda-Pineda, E. Ricci, L. Yin, J. Cohn, and N. Sebe, "Self-adaptive matrix completion for heart rate estimation from face videos under realistic conditions," in Proc. IEEE Conference on Computer Vision and Pattern Recognition, (CVPR), pp. 2396-2404, June 2016.

132. X. Li, J. Chen, G. Zhao, and M. Pietikäinen, "Remote heart rate measurement from face videos under realistic situations," in Proc. IEEE Conference on Computer Vision and Pattern Recognition, (CVPR), pp. 4264-4271, June 2014.

133. J. Chen, Z. Chang, Q. Qiu, X. Li, G. Sapiro, A. Bronstein, et. al., "RealSense = real heart rate: illumination invariant heart rate estimation from videos," in Proc. IEEE International Conference on Image Processing Theory, Tools and Applications (IPTA), pp. 1-6, December 2016.

134. S. Coleman et al., "A new pressure ulcer conceptual framework," Journal of Advanced Nursing, vol. 70, no. 10, pp. 2222-2234, 2014. 